\title{
BAYESIAN HIERARCHICAL MODELING OF COLORECTAL AND BREAST CANCER DATA IN MISSOURI
}

\author{
A Thesis presented to \\ the Faculty of the Graduate School \\ at the University of Missouri
}

In Partial Fulfillment

of the Requirements for the Degree

Doctor of Philosophy

by
JIANG DU

Dr. Dongchu Sun, Thesis Supervisor

MAY 2018 
The undersigned, appointed by the Dean of the Graduate School, have examined the dissertation entitled:

\section{BAYESIAN HIERARCHICAL MODELING OF COLORECTAL AND BREAST CANCER DATA IN MISSOURI}

presented by Jiang Du, a candidate for the degree of Doctor of Philosophy and hereby certify that, in their opinion, it is worthy of acceptance.

Dr. Dongchu Sun

Dr. Chong (Zhuoqiong) He

Dr. Athanasios (Sakis) Christou Micheas

Dr. Tieming Ji

Dr. Jeannette Jackson-Thompson 


\section{ACKNOWLEDGMENTS}

I must express my sincere and deepest gratitude to my advisor, Dr. Dongchu Sun

for his guidance, patience and expertise during my PhD study. Not only he helped me on the way of becoming a qualified statistician who can apply statistical knowledge into different fields, but also becoming an independent researcher who can discover new research opportunities in relevant fields.

I would also like to thank my mentor Dr. Chester Schmaltz and my supervisor Dr. Jeannette Jackson-Thompson at the Missouri Cancer Registry and Research Center. Without their continuous guidance and support, this work would have never come to fruition. In addition, I am also very grateful to the other members of my advisory committee: Dr. Tieming Ji, Dr. Chong (Zhuoqiong) He and Dr. Sakis Micheas, who guided me through various statistical courses and gave comments on my work. 


\section{TABLE OF CONTENTS}

ACKNOWLEDGMENTS ................ ii

LIST OF TABLES $\ldots \ldots \ldots \ldots \ldots \ldots \ldots$ vii

LIST OF FIGURES $\ldots \ldots \ldots \ldots \ldots \ldots$ viii

ABSTRACT .................... xi

\section{CHAPTER}

1 Introduction ...................... 1

1.1 Studies on colorectal cancer $\ldots \ldots \ldots \ldots \ldots$

1.1.1 CRC incidence and mortality rates . . . . . . . . . 2

1.1.2 CRCS prevalence estimates . . . . . . . . . . . 4

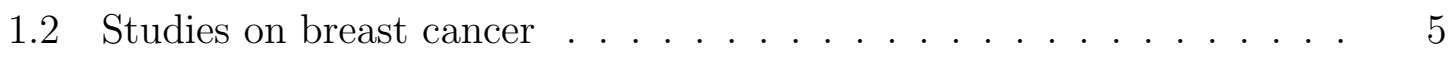

1.2.1 Breast cancer survival analysis . . . . . . . . . . 5

1.2.2 Breast cancer treatment delay . . . . . . . . . . . . 6

2 Jointly Modeling Colorectal Cancer Incidence and Mortality Rates on Misaligned Regions in Missouri . . . . . . . . . . . 10

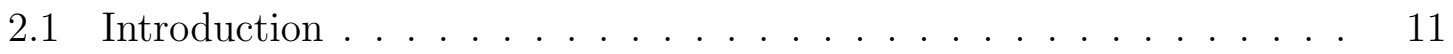

2.1.1 Review of spatial models for disease mapping . . . . . . . . 11

2.1.2 Multivariate disease mapping for misaligned regions . . . . . 15

2.1 .3 Data description . . . . . . . . . . . . . . 18

2.2 Model Setup . . . . . . . . . . . . . . . . . . . . . . . . 22

2.2 .1 Independent CAR Model . . . . . . . . . . . . . . . . . . 22 
2.2 .2 Joint Model . . . . . . . . . . . . . . . . . . . . . . . . . . . . 28

2.3 Model Fitting Results . . . . . . . . . . . . . . . . . . . . . 38

2.3 .1 Computation and convergence . . . . . . . . . . . 39

2.3.2 Sensitivity analysis of priors for variance parameters . . . . . 40

2.3 .3 Model selection . . . . . . . . . . . . . . . . . . . 43

$2.3 .4 \quad$ Numerical analysis . . . . . . . . . . . . . . . . . . 48

2.4 Discussion . . . . . . . . . . . . . . . . . . . . . . . . . 52

3 Using BRFSS data to estimate County-level Colorectal Cancer Screening Prevalence in Missouri . . . . . . . . . . . 56

3.1 Introduction . . . . . . . . . . . . . . . . . 57

3.1.1 Existing surveys covered CRCS in Missouri . . . . . . . . . 57

3.1 .2 Aim of the study . . . . . . . . . . . . . . . 59

3.1.3 Review of SAE methods for surveys . . . . . . . . . . 60

3.1 .4 Outline of the chapter $\ldots \ldots \ldots$. . . . . . . . 63

3.2 Methods . . . . . . . . . . . . . . . . . . . . . . 64

$3.2 .1 \quad$ Data . . . . . . . . . . . . . . . . . . 64

$3.2 .2 \quad$ Prevalence estimator . . . . . . . . . . . . . . 68

3.2 .3 Bayesian binomial regression . . . . . . . . . . . . . . . 69

3.2.4 Prior distributions on the regression parameters $\ldots \ldots \ldots 71$

3.2 .5 Selection of hyperpriors . . . . . . . . . . . . 73

3.2 .6 Estimates of CRCS prevalence . . . . . . . . . . . . . 74

3.3 Results . . . . . . . . . . . . . . . . . . . . . . . 74

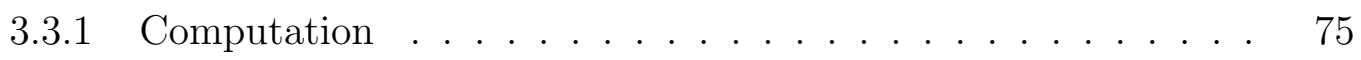


3.3 .2 Model evaluation . . . . . . . . . . . . . . . 76

3.4 Discussion . . . . . . . . . . . . . . . . . . . . . . . . . . . . 79

4 Hierarchical Models for Breast Cancer Survival in Missouri with Spatial, Age, and Stage Effects . . . . . . . . . . 81

4.1 Introduction . . . . . . . . . . . . . . . . . . . 82

4.2 Data and Models . . . . . . . . . . . . . . . . . . . . 84

4.2 .1 Data and notation . . . . . . . . . . . . . . 84

4.2 .2 Models . . . . . . . . . . . . . . . . . . . . . . . 87

4.3 Model Comparison and Results . . . . . . . . . . . . . . . 96

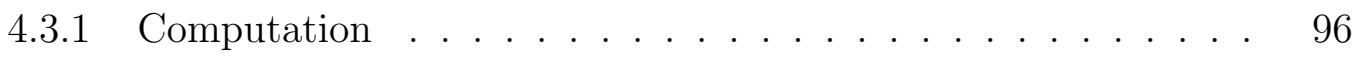

4.3 .2 Model comparison . . . . . . . . . . . . . . . . . . . . . 97

4.3 .3 Results . . . . . . . . . . . . . . . . . . . . . . . . . 9 98

4.4 Discussion . . . . . . . . . . . . . . . . . . . . . 105

5 A Scaled Spatio-temporal Model for Breast Cancer Treatment Delay108

5.1 Introduction . . . . . . . . . . . . . . . . . . 109

5.2 A scaled spatio-temporal model . . . . . . . . . . . . . . 111

5.2 .1 The BYM2 model . . . . . . . . . . . . . . . . . . . . . . . . 112

$5.2 .2 \quad$ A scaled RW2 model . . . . . . . . . . . . . . . . . 115

5.2.3 A scaled spatio-temporal interaction term . . . . . . . 116

5.3 Modeling the treatment delay $\ldots \ldots \ldots \ldots \ldots \ldots$

5.3 .1 Data overview . . . . . . . . . . . . . . . . . . . 117

5.3.2 Likelihoods and linear models . . . . . . . . . . . . . . 120

5.3 .3 Priors on regression parameters $\ldots \ldots \ldots \ldots \ldots \ldots$ 
5.3 .4 Identifiability constraints . . . . . . . . . . . . . . . . 124

5.3.5 Choice of hyperpriors: the penalised-complexity priors _ . . 125

5.4 Result analysis . . . . . . . . . . . . . . . . . . . 130

5.4 .1 Computation . . . . . . . . . . . . . 130

5.4 .2 Hyper parameter estimates . . . . . . . . . . . . . . 130

5.4 .3 Covariate Effects . . . . . . . . . . . . . . . . . 132

5.4.4 Predicted values on the original scale . . . . . . . . . 136

5.5 Simulation study . . . . . . . . . . . . . . . . . . 136

5.5 .1 A balanced simulation . . . . . . . . . . . . . 139

5.5 .2 A matched simulation . . . . . . . . . . . . 145

5.6 Discussion . . . . . . . . . . . . . . . . . . . . . . . . . . . . . 152

6 Summary and concluding remarks . . . . . . . . . . 155 APPENDIX

A Theorem and lemmas ................... 159

A.1 Positive definiteness requirements for $\gamma \ldots \ldots \ldots \ldots$

B Full conditional distributions $\ldots \ldots \ldots \ldots \ldots \ldots$

B.1 Full conditional distributions in Chapter $2 \ldots \ldots \ldots$

B.2 Full conditional distributions in Chapter $3 \ldots \ldots \ldots$

B.3 Full conditional distributions in Chapter $4 \ldots \ldots$. . . . . . . 169

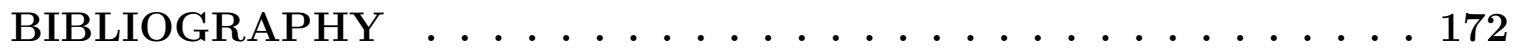

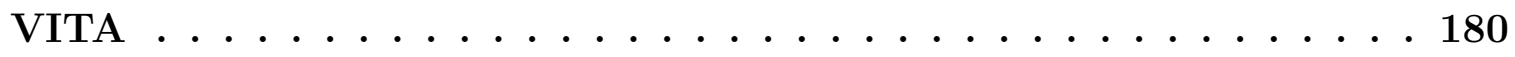




\section{LIST OF TABLES}

Table $\quad$ Page

2.1 Number of Regions for Each Data Category . . . . . . . . . . . . . 21

2.2 Posterior Mean and Standard Deviations of $\sigma_{i}^{2}$ and $\delta_{i} \ldots \ldots$. . . . . 42

2.3 DIC for the Independent, MarCAR and ConCAR models . . . . . . . 43

2.4 Posterior Quantities for Independent and Simplified ConCAR Models 49

3.12012 MO-BRFSS Regions in MO with Sample Sizes . . . . . . . . . . 67

3.2 Models with different covariates . . . . . . . . . . . . . . 75

3.3 Model Evaluation . . . . . . . . . . . . . . . . . . . 77

4.1 DIC for competing survival models . . . . . . . . . . . . . . . . 97

4.2 Posterior Means and Standard Deviations for Selected Parameters . . 101

5.1 Summary Statistics for Posterior Distributions of Hyper Parameters . 131

5.2 Truth vs. Estimates on Fixed Effects . . . . . . . . . . . . . . . . . . 142

5.3 Summary Statistics for Posterior Distributions of Hyper Parameters . 144

5.4 Truth vs. Estimates on Fixed Effects for Matched Simulation. . . . . 147

5.5 Summary Statistics for Posterior Distributions of Hyper Parameters in Matched Simulation. . . . . . . . . . . . . . . . . . . 152 


\section{LIST OF FIGURES}

Figure

2.1 Frequentist Incidence and Mortality Rates by Age, Gender and Year . 20

2.2 Prior Distribution of Spatial Variance Proportion $\phi \ldots \ldots \ldots$

2.3 Estimated Scale Reduction Factor $\hat{R} \ldots \ldots \ldots$

2.4 Posterior Mean of Cross Variable Precision Matrix for the Full ConCAR Model . . . . . . . . . . . . . . . . . . . 45

2.5 Posterior Mean of Cross Variable Precision Matrix for the Simplified ConCAR Model . . . . . . . . . . . . . . . . . . . . . . 46

2.6 Posterior Densities for Spatial Correlation Parameter $\rho_{i} \ldots \ldots \ldots$

2.7 Scatterplots of Posterior Means and Standard Deviations of Rates Based on the Independent and Simplified ConCAR . . . . . . . . 51

2.8 Smoothed Incidence and Mortality Rates by Age, Gender and Year . 53

2.9 Estimated Statewide Incidence and Mortality Rates for Each Year . . 54

3.12012 MO-BRFSS Regions. . . . . . . . . . . . . . . . . . . 65

3.22012 MO-BRFSS Sample Sizes Counties in Missouri . . . . . . . . . 66

3.3 Scatter Plots of Estimates from MO-BRFSS Versus CLS $\ldots \ldots .78$

3.4 Spatial Plots for CRCS Prevalence $\ldots \ldots \ldots \ldots \ldots$ 
4.1 Age Distribution Across Stages. . . . . . . . . . . . . . . 86

4.2 Stage Percentages for All Age Groups . . . . . . . . . . . . . 87

4.3 Proportion of $\Delta=1 \ldots \ldots \ldots \ldots \ldots \ldots$

4.4 Proportion of Localized, Regional and Distant Staged Patients within Each County . . . . . . . . . . . . . . . . 88

4.5 Trace Plots of Some Selected Parameters of Interest . . . . . . . . . 99

4.6 Posterior Histograms of the Parameters . . . . . . . . . . . . . . 100

4.7 Posterior Means of Spatial Effects . . . . . . . . . . . . . . . . 102

4.8 Scatter Plots of $v_{i}=\log \left(\lambda_{i}\right) \ldots \ldots \ldots \ldots \ldots$

4.9 Map of Average $v_{i}=\log \left(\lambda_{i}\right)$ for Each County With all Stages and Ages Combined . . . . . . . . . . . . . . . . . . . 104

4.10 Estimated Survival Functions for Patients Aged $\leq 34,60-64$, and 85+ in Years . . . . . . . . . . . . . . . . 106

5.1 Histogram of Treatment Delay Measured in Days. . . . . . . . . . 118

5.2 The Proportion of Zero Delay and the Average Positive Delayed Days for Each County in Missouri Over Years . . . . . . . . . . . . . 119

5.3 Density Plot for the PC Prior on Precision Parameter. . . . . . . . 128

5.4 Density Plot for the PC Prior on Precision Parameter. . . . . . . . 129

5.5 Box-plot for the Race and Stage Effects . . . . . . . . . . . . . 133

5.6 Estimates of the Age Effects . . . . . . . . . . . . . . . . . . 134

5.7 Estimates of the Temporal Effects . . . . . . . . . . . . . . . 135

5.8 Posterior Means of $\boldsymbol{\xi}^{(m)} \ldots \ldots \ldots \ldots \ldots \ldots \ldots$

5.9 Posterior Means of $\boldsymbol{\delta}^{(m)} \ldots \ldots \ldots \ldots \ldots \ldots$ 
5.10 Posterior Means of Predicted Same-day Treatment Probabilities and Number of Delayed Days. . . . . . . . . . . . . . . . . . 138

5.11 Scatter Plots for True vs. Estimates for Random Effects. . . . . . . . 143

5.12 Mean Absolute Deviance (MAD) from Estimates to True Values for Random Effects. . . . . . . . . . . . . . . . . . . . . . 143

5.13 Age Effects: Truth vs Estimates . . . . . . . . . . . . . . . . . . 148

5.14 Temporal Effects: Truth vs Estimates . . . . . . . . . . . . . . . 148

5.15 Comparison of Estimates for $\boldsymbol{\xi}^{(0)}$ and the True Values Used in Simulation.149

5.16 Comparison of Estimates for $\boldsymbol{\xi}^{(1)}$ and the True Values Used in Simulation.149

5.17 Comparison of Estimates for $\boldsymbol{\delta}^{(0)}$ and the True Values Used in Simulation.150

5.18 Comparison of Estimates for $\boldsymbol{\delta}^{(1)}$ and the True Values Used in Simulation.151 


\begin{abstract}
Data on cancer in the United States is collected through cancer registries. The Missouri Cancer Registry and Research Center (MCR-ARC) maintains a statewide cancer surveillance system and participate in research in support of the prevention of cancer and the reduction of the cancer burden among Missouri residents. We applied Bayesian hierarchical models to colorectal cancer (CRC) and breast cancer related data collected by the MCR-ARC. In the first project, $\mathrm{CRC}$ incidence and mortality rates in Missouri were studied with emphasis on different groups of people categorized by age, gender and county at diagnosis. The incidence and mortality data were aggregated into different spatial regions due to data confidential requirements, which was identified as a misaligned-region problem in multivariate disease mapping literature. The Marginally and Conditionally CAR models were built to address the problem. Later on, colorectal cancer screening (CRCS) prevalences were analyzed due to its importance to the early detection of CRC. We applied small area estimation techniques to produce county-level CRCS prevalences from the state-level Behavioral Risk Factor Surveillance System (BRFSS) data. The last two projects focused on breast cancer related data. One is about breast cancer survival analysis in Missouri with emphasis on detecting the spatial variation of survival time among counties in Missouri, after accounting for the differences in demographic and cancer stages. The other one is studying the disparities of breast cancer treatment delay with respect to patient's race, age, stage of cancer, county at diagnosis and year of diagnosis.
\end{abstract}




\section{Chapter 1}

\section{Introduction}

The topics in this dissertation are mainly about building Bayesian hierarchical models to analyze colorectal cancer (CRC) and breast cancer related data collected by the Missouri Cancer Registry and Research Center (MCR-ARC) and the Missouri Department of Health and Senior Services (DHSS). The MCR-ARC maintains a statewide cancer surveillance system and participate in research in support of the prevention of cancer and the reduction of the cancer burden among Missouri residents. It collects demographic, tumor and treatment information on more than 30,000 new cases of invasive cancer diagnosed among Missouri residents annually. The rich and high quality data base maintained by the MCR-ARC enable various research opportunities. For example, the $\mathrm{CRC}$ incidence and mortality rates are studied in Chapter 2. The breast cancer survival rates are studied in Chapter 4. In addition to the incidence, mortality and survival data, MCR-ARC also collects cancer treatment data. For example, we used the date of diagnosis and date of first treatment to calculate breast cancer treatment delay. A comprehensive spatio-temporal study on breast cancer treatment 
delay is in Chapter 5. As for DHSS, their Missouri Behavioral Risk Factor Surveillance System (BRFSS) data are used as a follow up study to investigate the CRC screening (CRCS) prevalences in Missouri. The CRCS serves an important role in the early detection of CRC, which is important to the survival of a CRC patient. The BRFSS is the nation's premier system of health-related telephone surveys that collect state data about U.S. residents regarding their health-related risk behaviors, chronic health conditions, and use of preventive services. Questions about CRCS are asked every two years and the state-level CRCS can be obtained. The CRCS prevalences in Missouri is studied in Chapter 3.

\subsection{Studies on colorectal cancer}

\subsubsection{CRC incidence and mortality rates}

$\mathrm{CRC}$ is the third most commonly diagnosed cancer and the third leading cause of cancer death in both men and women in the US. CRC incidence rates increased from 1975 through the mid-1980s (American Cancer Society, 2014), but have since decreased with the exception of a slight, unexplained bump in rates between 1996 and 1998. Declines have accelerated during the past few years. The incidence rates decreased by more than $4 \%$ from 2008 to 2010 . The large declines over the past decade have largely been attributed to the detection and removal of polyps as a result of increased CRC screening. As for the mortality rates, they have been decreasing along with the incidence rates. From 2001 to 2010, CRC mortality rates decreased by about $3 \%$ per year, compared to declines of about $2 \%$ per year in the 1990s. The declines in 
mortality rates have been attributed to improvements in treatment, changing patterns in $\mathrm{CRC}$ risk factors and screening. Both $\mathrm{CRC}$ incidence and mortality rates vary along with age, sex and race. Overall, $90 \%$ of new cases and $92 \%$ of deaths occur in people 50 years of age and older. Also, both rates are higher in men than in women. By race, both rates are highest in black men and women and lowest in Asian/Pacific Islander (API) men and women. For example, during 2006-2010, CRC incidence rates in blacks were about $25 \%$ higher than those in whites and about $59 \%$ higher than those in APIs, while the mortality rates in blacks are about 50\% higher than in whites and double those in APIs. In Chapter 2, the CRC incidence and mortality rates from years 1998 through 2012 in Missouri are studied with emphasis on different groups of people categorized by age, gender and county at diagnosis. For each group, Conditional Autoregressive Regression (CAR) models (Sun et al., 1999) can be built independently to study the spatial patterns. However, such models fail to consider possible correlations among the groups. Multivariate CAR models (Botella-Rocamora et al., 2015; Carlin et al., 2003; Gelfand and Vounatsou, 2003; Greco and Trivisano, 2009; Jin et al., 2007, 2005; Martinez-Beneito, 2013) can be used to account for those relationships, but those models are for data collected on the same geographical units. In our analysis, the incidence and mortality rates are aggregated differently based on counties due to data confidential requirements, which results in different spatial units for different groups of people. For multiple spatial variables with misaligned regions in the same area, Marginal and Conditional CAR models (Schmaltz, 2012) are used to fit the data. Both models introduce the correlations among different groups of people by assigning a certain covariance or precision matrix for the spatial random effects. 


\subsubsection{CRCS prevalence estimates}

As a follow up study of the CRC incidence and mortality rates, CRCS prevalences for counties in Missouri are analyzed in Chapter 3 due to its importance in the early detection of Colorectal cancer and reduces the mortality rate. It is recommended that people aged 50 and older should take CRCS regularly. However, not all people follow the guideline. The state-level CRCS prevalence can be estimated from the BRFSS data. Efforts to advocate CRCS are often conducted locally, often at the level of county or county equivalent. Knowing county-level CRCS prevalence can be important for making relevant policies. However, BRFSS does not provide countylevel CRCS prevalence estimates. In Chapter 3, the feasibility of producing countylevel estimates using BRFSS data are explored, with the use of small area estimation (SAE) techniques. Demographic information from both BRFSS and U.S. Census population file were used in our models. In addition, county attributes related to education levels and house incomes are used to improve the estimates. A random spatial effect is also added to capture other county attributes not included in the model. The 2012 Missouri BRFSS (MO-BRFSS) data is used as an example to get county-level CRCS prevalence estimates. To evaluate the results, estimates from 2011 Missouri County Level Study (MO-CLS), which is a BRFSS-like survey but collected hundreds of responses for each county in Missouri, are used as "true" values. The evaluation results indicate the inclusion of county attributes can improve the estimates significantly, but not the random spatial effect. The estimates from MO-BRFSS show similar patterns as those from MO-CLS but less accurate. 


\subsection{Studies on breast cancer}

\subsubsection{Breast cancer survival analysis}

Breast cancer is the most commonly diagnosed type of cancer and the second leading cause of cancer death among women in the United States. In 2017, breast cancer is estimated to account for $30 \%$ of all new cancers and $14 \%$ of cancer deaths for women (Siegel et al., 2017). As for its importance, breast cancer survival has been extensively studied. Breast cancer survival is closely related to stage at diagnosis. Survival is lower among women with a more advanced stage. Considering all races, 5-year relative survival was $98.9 \%$ for localized disease, $85.2 \%$ for regional disease and $26.9 \%$ for distant-stage disease based on patients diagnosed during 2007-2013 (Howlader et al., 2017). Aside from stage at diagnosis, age at diagnosis is also important when studying breast cancer survival rates. A Cox proportional hazards regression model was used (Rosenberg et al., 2005) to study the effect of age at diagnosis, where quadratic and linear age effects were used as covariates. The U-shaped curve from the model fitting results concluded poorer survival for the youngest and oldest age groups. Similar conclusions can be found in Wingo et al. (1998) and Yancik et al. (2001), which showed lower survival for women under 45 and over 55, respectively. Regardless of age, survival from breast cancer varies geographically in US by state (Jemal et al., 2017). For all stages and race combined, the 5-year relative survival rates varies from Mississippi (84.6\%) to New Hampshire (93.1\%) based on data between 2006 and 2012. For localized stage, the range is Alaska (94.8\%) to Colorado (99.0\%); for regional stage, the range is Mississippi (78.4\%) to New Hampshire (88.9\%); for distant stage, the range is Alaska (17.3\%) to Washington-Seattle (37.3\%). 
The relationship between cancer survival and individual characteristics has been mostly studied. For example, the effects of age, race, tumor size, tumor grade and disease stage were used when modeling breast cancer survival in Rosenberg et al. (2005). Those individual characteristics show significant effects when estimating breast cancer survival rates. However, some factors aside from the disease itself may also be important to the survival, such as the patient's income level, insurance status and quality of cancer treatments the patient receives. Those factors may be more or less represented by the difference among the regions in which the patient lives in. Chapter 4 assesses the small-area variation of breast cancer survival, which can be useful to detect if disparities exists in different areas so that proper medical and education interventions can be implemented. A Bayesian hierarchical model is used to model the survival time, which is assumed following a Weibull distribution. The rate parameter in Weibull distribution is further modeled by age, stage and county effects. The age effect is modeled non-linearly using a one dimensional smoothing spline. The county effect is modeled by CAR distribution. Several models are studied by considering different sets of parameter combinations. Based on the results, the non-linear age effect is confirmed, as well as the importance of including spatial random effect. However, the spatial random effect is relatively weak compared to age and stage effects.

\subsubsection{Breast cancer treatment delay}

The last part of our research was focused on breast cancer treatment delay. When a patient is diagnosed with breast cancer, there usually exists a delay before the treatment starts. The delay can be attributed to various reasons, including cost, scheduling, accessibility of medical care, etc. Various study exist discussing issues 
with breast cancer treatment delay. Bleicher et al. (2016) discussed the relationship between the breast cancer survival and time to surgery (TTS). They concluded that greater TTS is associated with lower overall and disease-specific survival, and a shortened delay is associated with benefits comparable to some standard therapies. McGee et al. (2013) concluded racial disparities in treatment delay exist among women less than 50 years old. Smith et al. (2013) found out longer treatment delay being one of the significant risk factors for shorter survival. In addition, they showed the treatment delay differs between racial/ethnic groups and public/private insurances.

The relationship between breast cancer survival and treatment delay has been established. However, few studies exist for a complete investigation on the disparities of treatment delay based on patients demographic information, stages of the disease. More importantly, it is unclear whether this disparities exist for people living in different areas and whether the treatment delay changed over recent years. A spatial analysis for the treatment delay can potentially include the attributes associated with different areas, like population size, house income, education level, amount of medical resources, etc. A temporal analysis can help us identify the changes of treatment delay over years, from which we can have a clear understanding about the current situation.

For reasons above, we analyzed the breast cancer treatment data obtained from Missouri Cancer Registry and Research Center. We define the treatment delay as the number of days between the date of diagnosis and the date of first treatment. The first treatment can be surgery, radiation, hormone therapy or biological response modifiers (BRM) therapy, whichever comes first. The data include all female breast cancer cases diagnosed from 1997 to 2014 in Missouri. We focused on investigating the relationship between treatment delay and several covariates. The covariates include patients' 
race, age at diagnosis, stage at diagnosis, year at diagnosis and county at diagnosis. Since the treatment delay is measured in days as non-negative integers, a Poisson regression framework can be a potential fit to our problem. However, we noticed that there are $27.37 \%$ of zeros for the treatment delay, which means some patients got same-day treatment. With the large amount of zeros, the Poisson distribution is not suitable anymore. To solve the problem, we used the Hurdle Poisson (HP) model for the response variable. It enables us not only to model the delayed days through a Poisson regression, but also to model the probability of getting same-day treatment (zero-delay) through a logistic regression. We are interested in studying the changes of zero-delay probability and positive-delay days across patients' age, race, stage, year of diagnosis (time) and county at diagnosis (space).

A Bayesian hierarchical model was built for our treatment delay data with the HP distribution being on the top level. With the presence of spatial (county) and temporal (year) covariates, a spatio-temporal model was included in our framework. We used a scaled spatio-temporal model for county and year effect, which was inspired by Riebler et al. (2016). Such model scales all random effect to have the same generalized variance, which makes the assignment of the hyper priors easier and more interpretable. The recent penalised-complexity (PC) (Simpson et al., 2017) priors were used as the hyper priors in our study. Markov Chain Monte Carlo (MCMC) is often used in Bayesian computation, as in our previous work. However, this time we used integrated nested laplace approximations (INLA) for approximate Bayesian inference (Rue et al., 2009). This method is promising since it reduces the Bayesian computation substantially, especially like in our case with a large data set. Additionally, a practical advantage of INLA is that it can be used within $\mathbf{R}$ (Team et al., 
2013) via the library R-INLA (Martino and Rue, 2009). Many latent Gaussian models have been implemented in R-INLA. With the use of INLA, we can get our Bayesian estimates without running long and complex MCMC algorithms. 


\section{Chapter 2}

\section{Jointly Modeling Colorectal Cancer Incidence and Mortality Rates on Misaligned Regions in Missouri}

In this chapter, the Colorectal cancer (CRC) incidence and mortality rates in Missouri were studied with emphasis on different groups of people categorized by age, gender and county at diagnosis. The incidence and mortality rates were aggregated differently based on counties due to data confidential requirements, which results in different spatial units for different groups of people. Marginal and Conditional CAR models were used to fit the data. Both models introduce the correlations among different groups of people by assigning a certain covariance or precision matrix for the spatial random effects. 


\section{$2.1 \quad$ Introduction}

\subsubsection{Review of spatial models for disease mapping}

Spatial modelling for cancer incidence and mortality rates falls under the broader category of disease mapping. Data associated with incidence and mortality rates are usually available together with disease counts at regional level (county, zip code, etc.) and are referred as area data. Small number of counts for some regions may provide unstable rate estimates. Many statistical models have been developed to produce better estimates by introducing dependence among neighboring regions. The counts are usually assumed to follow a Poisson distribution with its mean modeled by fixed and random effects. To be specific, let $y_{i}$ denote the counts for region $i(i=1, \ldots, n)$ distributed as

$$
y_{i} \mid r_{i} \sim \operatorname{Poisson}\left(\mu_{i}=e_{i} r_{i}\right) \quad \text { and } \log \left(\mu_{i}\right)=\log \left(e_{i}\right)+\log \left(r_{i}\right)
$$

where $e_{i}$ is a constant and $r_{i}$ is the rate for region $i$. When $e_{i}$ is the expected number of cases, $r_{i}$ can be interpreted as the relative risk. When $e_{i}$ is the population size, $r_{i}$

will just be the rate. Depending on the specification of $\log \left(r_{i}\right)$, different models can be defined. Let $\boldsymbol{r}=\left(r_{1}, \ldots, r_{n}\right)^{\prime}$, then a commonly used form is

$$
\log (\boldsymbol{r})=\boldsymbol{X} \boldsymbol{\beta}+\boldsymbol{u}
$$

where $\boldsymbol{\beta}$ are the fixed effect parameters, $\boldsymbol{X}$ is the design matrix and $\boldsymbol{u}=\left(u_{1}, \ldots, u_{n}\right)^{\prime}$ denote the random spatial effects on $n$ regions. A Conditional Autoregressive (CAR) 
prior (Sun et al., 1999) on $\boldsymbol{u}$ is usually used to account for spatial dependence, i.e.

$$
\boldsymbol{u} \mid \delta, \boldsymbol{B} \sim N_{n}\left(\mathbf{0}, \delta \boldsymbol{B}^{-1}\right), \quad \boldsymbol{B}=\boldsymbol{D}-\rho \boldsymbol{W},
$$

where $N_{n}$ denotes an $n$-dimensional normal distribution, $\boldsymbol{D}$ is an $n \times n$ diagonal matrix with diagonal elements $d_{i}$ being the number of neighbors for region $i, \boldsymbol{W}$ is the adjacency matrix $\left(\boldsymbol{W}_{i i}=0 ; \boldsymbol{W}_{i i^{\prime}}=1\right.$ if region $i$ is a neighbor to region $i^{\prime}$ and $\boldsymbol{W}_{i i^{\prime}}=0$ otherwise), $\delta$ is a spatial variance parameter and $\rho$ controls the strength of spatial autocorrelation. Under this CAR prior specification, the conditional distribution of $u_{i}$ given all the others is:

$$
u_{i} \mid u_{-i}, \delta, \rho_{i} \sim N\left(\rho \frac{1}{d_{i}} \sum_{i \sim i^{\prime}} u_{i^{\prime}}, \frac{\delta}{d_{i}}\right),
$$

where $u_{-i}$ stands for the spatial effects on all the other regions except region $i$. The CAR prior (2.3) reduces to the intrinsic CAR (ICAR) model (Besag et al., 1991) when $\rho=1$, or an independent model when $\rho=0$.

Another similar approach is to propose a Gaussian convolution prior for $\boldsymbol{u}$ (Besag et al., 1991) which separate $\boldsymbol{u}$ into two independent components: one with ICAR prior and the other one with unstructured Gaussian independent prior, which is called the BYM model in literature. This model can account for both spatial dependency and region-wide heterogeneity. The idea of bringing into unstructured variability was also implemented in Leroux et al. (2000) for prior of $\boldsymbol{u}$ as

$$
\boldsymbol{u} \sim N_{n}\left(\mathbf{0}, \delta(\lambda \boldsymbol{Q}+(1-\lambda) \boldsymbol{I})^{-1}\right)
$$


and in Dean et al. (2001) as

$$
\boldsymbol{u} \sim N_{n}\left(\mathbf{0}, \delta\left(\lambda \boldsymbol{Q}^{-}+(1-\lambda) \boldsymbol{I}\right)\right)
$$

where $\boldsymbol{I}$ is the $n$ dimensional identity matrix. The $i$-th diagonal element of $\boldsymbol{Q}$ is the number of neighbors for region $i$ and the elements in $i$-th row out of the diagonal are -1 if the corresponding regions are neighbors and 0 otherwise. The parameter $\delta$ still represents the spatial dispersion while $\lambda$ determines the variance partition between structured and unstructured variation. Note that $\boldsymbol{Q}^{-}$is the Moore-Penrose generalized inverse.

In the case of multiple spatial effects (variables), not only the spatial correlation within each variable can be helpful for estimation, but also the modelling for cross covariance among variables can improve the estimates when multiple variables are correlated. The multivariate disease mapping literature has covered topics regarding to jointly modelling for several diseases. Let $u_{i j}$ denote the spatial effect at region $i$ for variable $j$, where $i=1, \ldots, n$ stands for $n$ regions and $j=1, \ldots, p$ stands for $p$ diseases. Then $\boldsymbol{u}_{j}=\left(u_{1 j}, \ldots, u_{n j}\right)^{\prime}$ is the random effects for disease $j$ and we collect all the random effects into vector $\boldsymbol{u}=\left(\boldsymbol{u}_{1}^{\prime}, \ldots, \boldsymbol{u}_{p}^{\prime}\right)^{\prime}$. Gelfand and Vounatsou (2003) and Carlin et al. (2003) generalized the univariate CAR model (2.3) to jointly modeling $p$ variables together by

$$
\boldsymbol{u} \sim N_{n p}\left(\mathbf{0},\{\boldsymbol{\Lambda} \otimes(\boldsymbol{D}-\rho \boldsymbol{W})\}^{-1}\right)
$$

where $\boldsymbol{\Lambda}$ is a $p \times p$ positive definite matrix which is interpreted as the non-spatial cross variable precision matrix between $p$ diseases and " $\otimes$ " denotes the Kronecker prod- 
uct. This model is often denoted as $\operatorname{MCAR}(\rho, \boldsymbol{\Lambda})$. One disadvantage of $\operatorname{MCAR}(\rho, \boldsymbol{\Lambda})$ is that it assumes the same spatial process for all $p$ variables. To extend it, a $\operatorname{MCAR}\left(\rho_{1}, \ldots, \rho_{p}, \boldsymbol{\Lambda}\right)$ model is developed as

$$
\boldsymbol{u} \sim N_{n p}\left(\mathbf{0},\left\{\operatorname{diag}\left(\boldsymbol{A}_{1}, \ldots, \boldsymbol{A}_{p}\right)\left(\boldsymbol{\Lambda} \otimes \boldsymbol{I}_{n \times n}\right) \operatorname{diag}\left(\boldsymbol{A}_{1}, \ldots, \boldsymbol{A}_{p}\right)^{\prime}\right\}^{-1}\right)
$$

where $\boldsymbol{A}_{j} \boldsymbol{A}_{j}^{\prime}=\boldsymbol{D}-\rho_{j} \boldsymbol{W}, j=1, \ldots, p$. This model allows different spatial effect for different variables through different $\rho_{j}$ s. However, the cross covariance matrices in off diagonal blocks are completely determined by the diagonal blocks, in which case the cross covariance matrices are not smoothed explicitly. Jin et al. (2005) proposed a more flexible generalized MCAR (GMCAR) by sequentially conditioning one variable on the rest to have a CAR prior for each conditional distribution. For example, if we only have $p=2$ variables, a GMCAR model assumes the conditional distribution of $\boldsymbol{u}_{1} \mid \boldsymbol{u}_{2}$ as $\boldsymbol{u}_{1} \mid \boldsymbol{u}_{2} \sim N_{n}\left[\left(\eta_{0} \boldsymbol{I}+\eta_{1} \boldsymbol{W}\right) \boldsymbol{u}_{2}, \delta_{1}\left(\boldsymbol{D}-\rho_{1} W\right)^{-1}\right]$ and the marginal distribution of $\boldsymbol{u}_{2}$ as $\boldsymbol{u}_{2} \sim N_{n}\left[\mathbf{0}, \delta_{2}\left(\boldsymbol{D}-\rho_{2} W\right)^{-1}\right]$. However, this GMCAR models suffers the ordering problem for these $p$ variables when building up the conditional distributions. Later on, Jin et al. (2007) proposed a more general order-free $\operatorname{MCAR}(\boldsymbol{B}, \boldsymbol{\Gamma})$ model based on linear model of coregionalization. In their framework, they model the spatial effects for each disease as

$$
\boldsymbol{u}=\left(\boldsymbol{A} \otimes \boldsymbol{I}_{n}\right) \boldsymbol{\phi},
$$

where $\boldsymbol{\phi}=\left(\boldsymbol{\phi}_{1}^{\prime}, \ldots, \boldsymbol{\phi}_{p}^{\prime}\right)^{\prime}$ and $\boldsymbol{\phi}_{j}=\left(\phi_{1 j}, \ldots, \phi_{n j}\right)^{\prime}$ represent latent spatial process for each disease which follow multivariate spatial process. The matrix $\boldsymbol{A}$ linearly transfer the latent spatial process $\phi$ to the variable of interest $\boldsymbol{u}$. For the dependent and not identical latent processes $\boldsymbol{\phi} \sim N_{n p}\left[\mathbf{0},\left(\boldsymbol{I}_{p} \otimes(\boldsymbol{D}-\boldsymbol{B}) \otimes \boldsymbol{W}\right)^{-1}\right]$ where $\boldsymbol{B}$ is a $p \times p$ 
symmetric matrix whose diagonal elements $b_{k k}$ represent the spatial correlation for variable $\phi_{k}$ and the off-diagonal elements $b_{k l}=b_{l k}$ account for cross correlations among different $\phi_{j}$ s, then the joint distribution of $\boldsymbol{u}$ is

$$
\boldsymbol{u} \sim N_{n p}\left(\mathbf{0},\left(\boldsymbol{A} \otimes \boldsymbol{I}_{n}\right)\left(\boldsymbol{I}_{p} \otimes(\boldsymbol{D}-\boldsymbol{B}) \otimes \boldsymbol{W}\right)^{-1}\left(\boldsymbol{A} \otimes \boldsymbol{I}_{n}\right)^{\prime}\right)
$$

This formulation gives $\boldsymbol{\Lambda}=\boldsymbol{A} \boldsymbol{A}_{1}^{\prime}$ as the conditional covariance matrix. The application of linear model coregionalization introduces much more possibilities to construct more general and complicated models. For example, Greco and Trivisano (2009) released the symmetric requirements for matrix $\boldsymbol{B}$; Martinez-Beneito (2013) proposed a $\boldsymbol{Q R}$-based multivariate disease mapping model, which encompasses a diverse range of statistical models for multivariate disease mapping while providing faster computation strategies; Botella-Rocamora et al. (2015) proposed an alternative reformulation, which is called $\boldsymbol{M}$-based models and can model more diseases (up to 21 diseases in their application) with WinBUGS under a reasonable amount of time. The models in Martinez-Beneito (2013) and Botella-Rocamora et al. (2015) provide unifying tools to model multiple diseases with WinBUGS, which is more accessible by most people.

\subsubsection{Multivariate disease mapping for misaligned regions}

The multivariate disease models mentioned in Section 2.1.1 provide us wide choices for the needs to model multiple spatial effects with accounting for both within and between variable correlations. Notice that for all the multivariate disease models, the underlying spatial structure are the same for all the variables. For example, when we are modeling several types of cancer, all cancers are measured on the same areas (like 
each county in a certain state). However, if the spatial structures of the variables are different from each other, those multivariate disease models can not be applied directly. For example as in our application, due to confidentiality and result stability requirements, cancer incidence and mortality data can only be released to the public when the case count reaches a certain threshold. Consequently, data suppression rules often result in missing values in county-level studies where cancer incidence or mortality counts are rather small for a given time period and a given group of people. One way to solve data suppression issue is using the original data and release the results accordingly. However, it is not always possible to have them. Moreover, a large amount of zeros or small counts may potentially make our rate estimates unstable with large variance. Alternatively, we can spatially aggregate the original data into larger regions in order to meet the suppression threshold as well as stabilize our estimates. Different types of cancer will have different aggregation results. Also, different groups of people manifesting the same type of cancer will also have different aggregation results. This kind of problem can be stated as a misaligned area problem. In spatial statistics literature, spatial misalignment often refers to the following two kinds of problems as discussed in Banerjee et al. (2014). For areal unit data, the purpose might be simply to understand the variable's distribution at a new level of spatial aggregation (the modifiable areal unit problem); for data modeled through a spatial process, we would envision block averaging at different spatial scales (the change of support problem). Gotway and Young (2002) contains a nice discussion of the models for incompatible spatial data. However, those models are not helpful in the literature of multivariate disease mapping problem with misaligned areas.

A straightforward way of applying existing multivariate disease mapping models 
is generating atoms by intersecting (i.e. further partitioning regions to the same resolution) all misaligned regions so that the multivariate model can be applied to these common atoms at finer scales. Then a linear combination of the atoms in corresponding regions can be formed so that we can derive new distributions for all variables with misaligned regions. Although it can provide a solution to the misaligned problem, there are several drawbacks. Firstly, we may prefer each variable itself marginally or conditionally having a CAR prior (2.3) since that is what happens if we are modeling the variables separately. But a linear combination of hidden variables on those atoms may not guarantee such property. Secondly, if a region itself already has a small case count, further partition into small atoms may assign extensive amounts of zeros for the hidden variables at atom level. Although one may not need to estimate those hidden variables, the extensive zeros may potential produce unstable estimates. Last but not least, we want to incorporate additional information when building the cross covariance matrices, which is not easily achievable for such linear combination. For example, considering we have two overlapped regions from two different cancers, then it is reasonable to assume the correlation between those two regions is related to the population size on the overlapped part. If no people live on the overlapped part, we may just assume zero correlation between those two regions. On the other hand, if all people lives on the overlapped part and the non-overlapped parts are so rural that have zero population, then we may expect strong correlations between those areas, which is equivalent to the perfectly aligned case. The population size may provide crucial information for cross covariance matrices, which can be better than just letting the data to reveal the correlations itself. One may also consider applications in environmental studies, the area size of the overlapped regions may 
contains some useful information. Depending on the type of data, different outside information can be incorporated.

Schmaltz and Sun (2010) and Schmaltz (2012) developed two multivariate distributions for misaligned regions with the use of overlapping matrices: the marginal CAR model (MarCAR) and conditional CAR model (ConCAR). The idea is to model the spatial covariance or precision matrix among all variables together by constructing their overlapping matrices based on their spatial structures. The structure of the overlapping matrices can incorporate outside information such as area size, population size, etc. The strength of cross correlations among variables can be controlled by some other parameters together with the overlapping matrices. In this paper, we will use the MarCAR and ConCAR models to investigate CRC incidence and mortality rates in Missouri for different groups of people based on age, gender and county at diagnosis. Details of the models will be displayed in later sections.

\subsubsection{Data description}

The CRC incidence data for Missouri was provided by the Missouri Cancer Registry and the mortality data was provided by Missouri Department of Health and Senior Services. For both data sets, we only selected malignant cases with known genders from years 1998 through 2012. We also included gender, age and county of residence at diagnosis or death as variables. For this study, we only focused on people 50 years of age or older, since this group accounts for over $90 \%$ of CRC cases and 50 is the recommended screening age for people at average risk. We divided both datasets into 5 time periods: 1998-2000, 2001-2003, 2004-2006, 2007-2009 and 2010-2012. Also, we divided people into 2 age groups, 50-64 and 65 and over. The spatio-temporal 
analysis is ideally based on the 115 locations (114 counties plus the City of St. Louis) and the 5 time periods, but the confidentiality rule must be taken into account. For the incidence data, cells (county-age-year combinations) with counts less than 6 need to be suppressed and for the mortality data, counts less than 10 need to be suppressed. To work around this, spatial aggregation was performed by grouping nearby counties for each age-gender combination. For the convenience of studying temporal patterns, the aggregations stay the same across the 5 time periods. Figure 2.1 shows the frequentist estimates of incidence and mortality rates on the aggregated regions by age, gender and year. The yearly rates per 100,000 people were calculated as

$$
\frac{\text { number of cases }}{\text { population size }} \times \frac{100000}{3} \text {. }
$$

Since we combined 3 years of cases in the numerator, and the population is the midyear population of the middle year (for example, the population size for 1998-2000 is that on 1 July 1999), we divided the rates by 3 to get the yearly rates. There are other choices for the population size, for example, we can use the average population size over 3 years, or simply use the sum of 3 years' population sizes without dividing by 3 . Based on our experience, there is no big difference among the choices of population size in terms of model fitting results. In Figure 2.1 there are four subfigures corresponding to the incidence rates of people $50-64,65+$ and mortality rates of people $50-64$ and $65+$. Within each sub figure, the upper five maps stand for males and the lower ones stand for females, with 5 time periods going from left to right. Notice that the spatial aggregation results are different between incidence and mortality data. Moreover, the partitions are different between males and females. However, for each age-gender combination, the spatial aggregation remains the same across the 5 time periods. In 


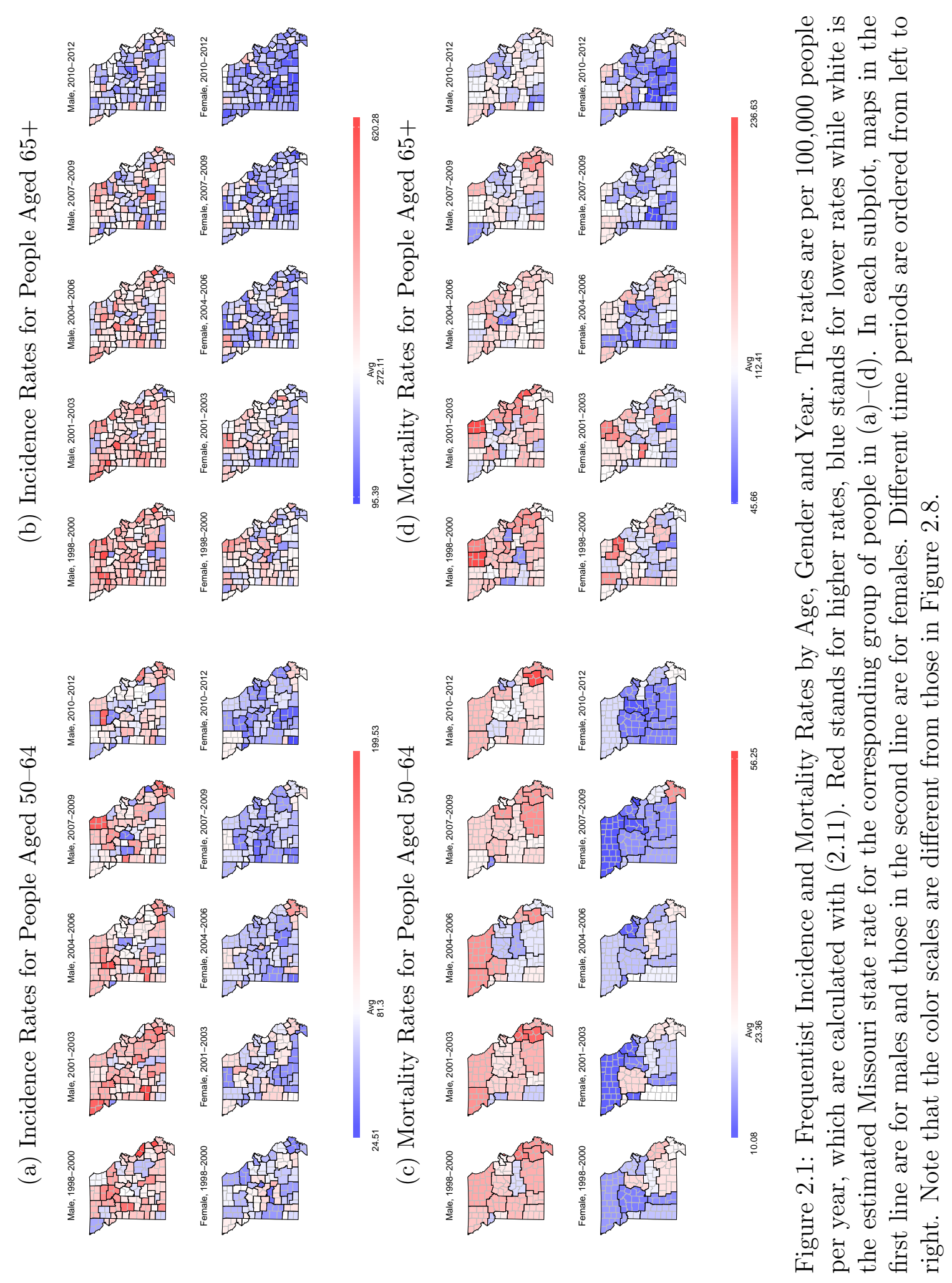


the end, we have a different number of regions for each data-age-gender category as shown in Table 2.1. Given the smaller number of cases of mortality data compared Table 2.1: Number of Regions for Each Data Category

\begin{tabular}{llllc}
\hline & Data & Gender & Age & Number of regions \\
\hline 1 & Incidence & Male & $50-64$ & 58 \\
2 & Incidence & Female & $50-64$ & 48 \\
3 & Incidence & Male & $65+$ & 92 \\
4 & Incidence & Female & $65+$ & 91 \\
5 & Mortality & Male & $50-64$ & 19 \\
6 & Mortality & Female & $50-64$ & 15 \\
7 & Mortality & Male & $65+$ & 39 \\
8 & Mortality & Female & $65+$ & 42 \\
\hline
\end{tabular}

to incidence data and a stricter suppression rule, the resulting regions are fewer in number but larger in size than those from the incidence data. Our goal of this analysis is to find the patterns in both space and time for the 8 categories of data in Table 2.1 .

The rest of the chapter is as follows. Section 2.2 discusses the model structure of the Independent CAR, MarCAR and ConCAR models specifically for our application to Missouri CRC data. Section 2.3 contains the computation strategy to fit those models as well as the model fitting results, emphasized on the comparison between Independent CAR and ConCAR models. Section 5.6 include discussions and comments on our paper. 


\subsection{Model Setup}

\subsubsection{Independent CAR Model}

The 8 categories of data in Table 2.1 are denoted as variable 1 to 8 . Variable 1 to 4 stand for the incidence data while variable 5 to 8 stand for the mortality data. Since we are interested in studying the spatio-temporal patterns for all 8 variables, an easy solution is to build 8 independent disease mapping models with spatial and temporal effects as covariates. Let $y_{i t j}$ be the incidence or mortality case count for the $i^{\text {th }}(i=1, \ldots, I)$ variable during time period $t(t=1, \ldots, T)$ in region $j\left(j=1, \ldots, J_{i}\right)$, where $I$ is number of variables of interest, $T$ is number of time periods and $J_{i}$ is the number of regions for variable $i$. In our case, we have $I=8$ variables, $T=5$ time periods, $\left(J_{1}, J_{2}, \ldots, J_{I}\right)$ are the number of regions in Table 2.1 and we let $J_{+}=\sum_{i=1}^{I} J_{i}$ be the total number of regions. For vector notation, we use $\boldsymbol{y}_{i t}=\left(y_{i t 1}, y_{i t 2}, \ldots, y_{i t J_{i}}\right)^{\prime}$, $\boldsymbol{y}_{i}=\left(\boldsymbol{y}_{i 1}^{\prime}, \boldsymbol{y}_{i 2}^{\prime}, \ldots, \boldsymbol{y}_{i T}^{\prime}\right)^{\prime}$ and $\boldsymbol{y}=\left(\boldsymbol{y}_{1}^{\prime}, \boldsymbol{y}_{2}^{\prime}, \ldots, \boldsymbol{y}_{I}^{\prime}\right)^{\prime}$. Also, we use $n_{i t j}$ as the population size associated with $i^{\text {th }}$ variable during time period $t$ in region $j$.

\section{A spatio-temporal model for count data.}

Following the disease mapping model (2.1), we assumed the case counts following a Poisson distribution:

$$
y_{i t j} \mid p_{i t j} \sim \operatorname{Poisson}\left(n_{i t j} p_{i t j}\right)
$$

and we used a linear mixed model:

$$
\eta_{i t j}=\log \left(p_{i t j}\right)=\alpha_{i}+\beta_{i} s_{t}+u_{i j}+\epsilon_{i t j},
$$


where $n_{i t j}$ is the population size for variable $i$, time $t$ and region $j, p_{i t j}$ is the corresponding incidence/mortality rate, $\alpha_{i}$ stands for the variable specific intercept which accounts for the effect of different age-gender combinations. In the linear mixed models (2.13), we assumed linear time trends for the log-rate $\eta_{i t j}$, so $\beta_{i}$ is the slope parameter for variable $i$ and $s_{t}$ is the time covariates generated as $s_{t}=(t-3) / \sqrt{2.5}$, $t=1, \ldots, 5$, which is standardized from $1,2, \ldots, 5$ since there are 5 time periods. Flat priors were used for $\alpha_{i}$ and $\beta_{i}$ as

$$
\alpha \sim N\left(0,10^{4}\right), \quad \text { and } \quad \beta \sim N\left(0,10^{4}\right)
$$

since we have little information about those parameters. The random effect $u_{i j}$ was used to account for the spatial dependence, so the vector $\boldsymbol{u}_{i}=\left(u_{i 1}, \ldots, u_{i J_{i}}\right)^{\prime}$ collects the spatial effects for variable $\boldsymbol{y}_{i}$, which are assumed to stay constant over time and independent with $\alpha_{i}$ and $\beta_{i}$. We used the same CAR prior (2.3) for the spatial effect $\boldsymbol{u}_{i}$

$$
\boldsymbol{u}_{i} \mid \delta_{i}, \boldsymbol{B}_{i} \sim N_{J_{i}}\left(\mathbf{0}, \delta_{i} \boldsymbol{B}_{i}^{-1}\right) \quad \text { and } \quad \boldsymbol{B}_{i}=\boldsymbol{D}_{i}-\rho_{i} \boldsymbol{W}_{i}
$$

where $\delta_{i}$ is the spatial variance parameter for variable $i, \boldsymbol{D}_{i}$ and $\boldsymbol{W}_{i}$ are the $J_{i} \times J_{i}$ diagonal matrix with number of neighbors on diagonals and the $J_{i} \times J_{i}$ adjacency matrix, which are defined the same as in (2.3) other than adding subscript $i$ to denote variable $i$. The difference between our specification (2.13) and the common form (2.2) is the additional unstructured over-dispersion (error) term $\epsilon_{i t j}$, which measures any other left-over variation. This idea is similar to the convolution prior (Besag et al., 1991) for the spatial effect. In our analysis, we put random normal priors on $\epsilon_{i t j}$ with 
variance $\sigma_{i}^{2}$ for variable $i$ :

$$
\epsilon_{i t j} \sim N\left(0, \sigma_{i}^{2}\right)
$$

Note that in our analysis, we used the simplest linear time trends assumption, as well as independence between space and time. That is because our data only have 5 time periods and by inspecting the plots of frequentist estimates for $\log \left(\eta_{i t j}\right)$ aggregated at the state level for each variable, a linear relationship is enough to describe the change over time. However, in general we can employ non-linear time series models as well as build structured interactions between space and time. For example, we can use $\left(\beta_{i}+v_{i j}\right) s_{t}$ instead of just $\beta_{i} s_{t}$, where $v_{i j}$ is another spatial effect which affects the slope parameter. Doing such will add space-time interactions. See Sun et al. (2000) for details. To release the linear assumption, we can put autoregressive priors on the time effect. For example, Zhang et al. (2006) used a second order intrinsic autoregressive prior $\operatorname{IAR}(2)$ for the time effects, which is equivalent to a second order smoothing spline. Ugarte et al. (2014) mentioned that first or second order random walk process can be applied. Moreover, the applications in Ugarte et al. (2014) gave structured covariance matrix to the over dispersion term ( $\epsilon_{i t j}$ in our case) by the Kronecker product between the precision matrices from space and time effects. Such structure will also introduce the interactions between space and time.

\section{Choice of hyper priors.}

To conduct full Bayesian analysis, we need to assign prior distributions to the rest hyper parameters. To ensure positive definiteness of $\boldsymbol{B}_{i}$ in (2.15), we need the spatial autocorrelation parameter $\rho_{i}$ to be in the range of $\left(1 / \lambda_{i}^{(\min )}, 1\right)$, where $\lambda_{i}^{(\min )}$ is the

smallest eigenvalue of $\boldsymbol{D}_{i}^{-1 / 2} \boldsymbol{W}_{i} \boldsymbol{D}_{i}^{-1 / 2}$. It can be proved that $\lambda_{i}^{(m i n)}$ is always negative 
(Sun et al., 1999). Since in practice we expect positive correlations with neighbors in the context of cancer incidence and mortality, we restrict $\rho$ to be positive. Thus one good choice for the prior of $\rho$ is

$$
\rho \sim \operatorname{Unif}(0,1) .
$$

The prior distributions for the variance component $\sigma_{i}^{2}$ and $\delta_{i}$ need to be chosen with care. In the literate of disease mapping, Inverse Gamma (IG) priors for those variance parameters have been used extensively (Schmaltz (2012), Sun et al. (2000) and Ugarte et al. (2014) for example). However, in most cases the shape and rate parameters of IG priors are chosen rather ad hoc and need to be tuned in different applications. In situations where weakly spatial information presented, the choice of the shape and rate parameter may affect the posterior inference for the variance parameter a lot. Our experience on fitting models in this paper has shown that the posterior means of $\sigma_{i}^{2}$ and $\delta_{i}$ can be quite sensitivity to difference choices of the IG priors. Riebler et al. (2016) recommended assign hyper priors after scaling the graph represented by the neighborhood structure. By scaling the graph, the priors specifications can be chosen more intuitively and they recommended the penalisedcomplexity priors (Simpson et al., 2017). However, our graph (precision matrix) $\boldsymbol{B}_{i}$ in (2.15) cannot be scaled since it is dependent on $\rho_{i}$. In the end, we followed the recommendations in Gelman et al. (2006) and chose Half-Cauchy (HC) priors for the standard deviation $\sigma_{i}$ :

$$
\left[\sigma_{i} \mid \zeta\right] \propto \frac{1}{\sigma_{i}^{2}+\zeta^{2}},
$$

where $\zeta$ is the scale parameter and also the median of $\mathrm{HC}(\zeta)$ distribution. The 
parameter $\zeta$ can be chosen relatively large in order to provide weakly informative priors. For example, if we use the frequentist estimates of $p_{i t j}$ and calculate the standard deviations of $\eta_{i t j}=\log \left(p_{i t j}\right)$ for each variable $i$, the largest values is around 0.31 for our data. Thus a choice $\zeta=1$ would be sensible since it not only covers most of the possible values for $\sigma_{i}$, but also exclude unrealistic large standard deviations. Note that Half-t distributions with 3 or 4 degrees of freedom and carefully chosen scale parameter can also be used if one thinks the $\mathrm{HC}$ prior is too weak.

When choosing the prior distribution for $\delta_{i}$, we incorporate the noise-to-signal ratio idea. Let

$$
\omega_{i}=\frac{\sigma_{i}^{2}}{\delta_{i}} .
$$

be the ratio between noise variance $\sigma_{i}^{2}$ and signal variance $\delta_{i}$. Sun and Speckman (2008) proved that in linear mixed models, a proper prior is need for $\omega_{i}$ if we use the improper $1 / \sigma_{i}^{2}$ on the noise variance $\sigma_{i}^{2}$, while it is unclear if the propriety still hold for generalized linear mixed models as in our case. However, since we used proper HC priors on $\sigma_{i}$, posterior propriety can be guaranteed as long as we use a proper prior on $\omega_{i}$. We adapted the idea from Cheng and Speckman (2012), where they used a scaled Pareto prior on $\omega_{i}$ in spline settings and is given by

$$
\left[\omega_{i} \mid a\right]=\frac{a}{\left(a+\omega_{i}\right)^{2}}, \quad \omega_{i}>0 .
$$

The use of noise-to-signal ratio has the benefit of introducing dependence between unstructured error $\boldsymbol{\epsilon}$ and spatially structured random effect $\boldsymbol{u}$ through their variance 


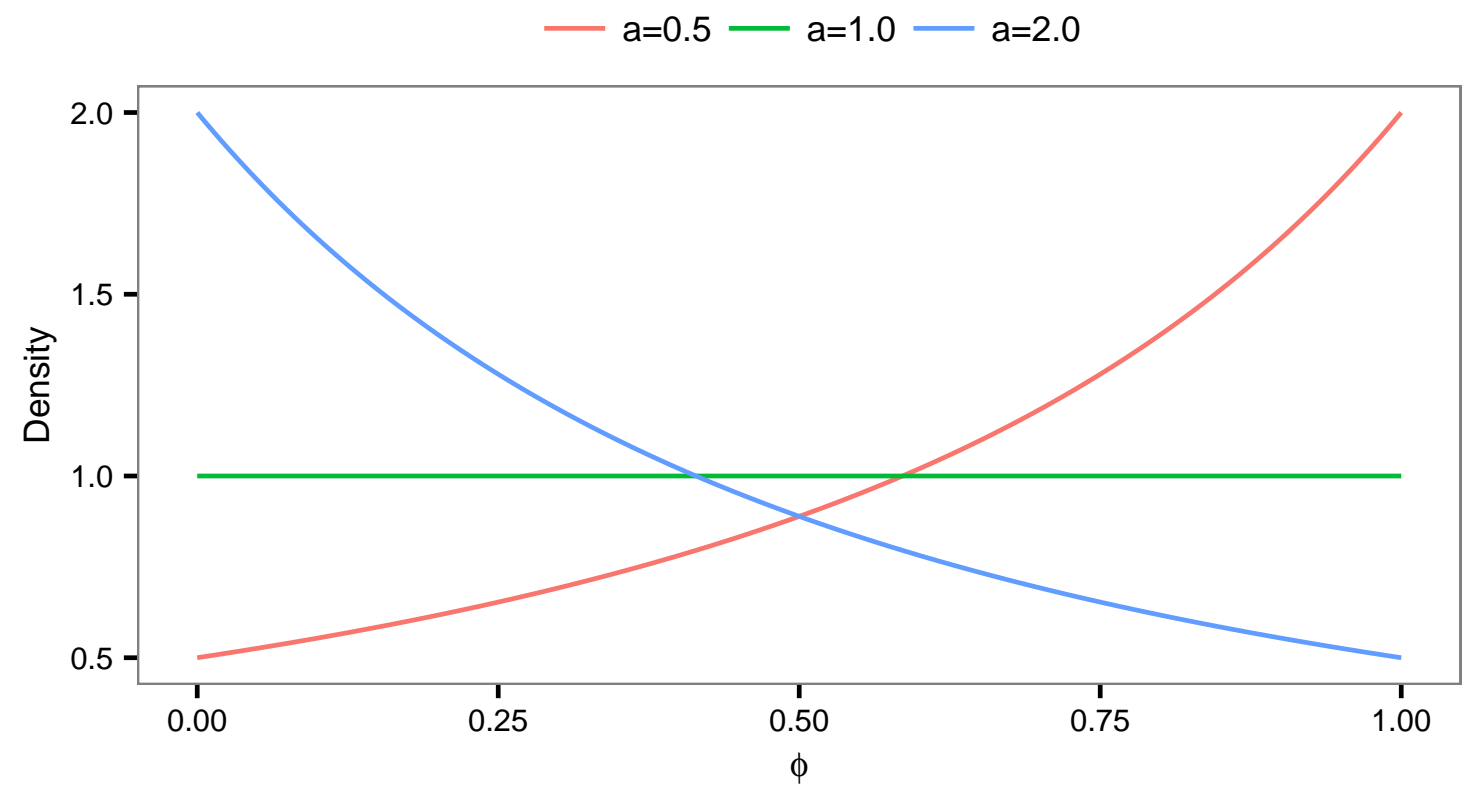

Figure 2.2: Prior Distribution of Spatial Variance Proportion $\phi$ for Different Values of $a$.

parameter. If we define the proportion of spatial variance as

$$
\phi_{i}=\frac{\delta_{i}}{\delta_{i}+\sigma_{i}^{2}}
$$

then the $\phi_{i}$ is distributed as

$$
\left[\phi_{i} \mid a\right]=\frac{a}{\left[(a-1) \phi_{i}+1\right]^{2}}
$$

In this sense, the total variation is separated into the structured spatial variation with proportion $\phi_{i}$ and the unstructured random noise with proportion $1-\phi_{i}$. This idea is similar to the Dean et al. (2001) prior in (2.6). Figure 5.4 shows the density plot of $\phi$ with $a=0.5,1$ and, 2. When $a=1, \phi \sim \operatorname{Uniform}(0,1)$; when $a<1$, more 
weight is put on the spatial random effect; when $a>1$, more weight is put on the unstructured random effects. In our application, we choose $a=1$ and sensitivity analysis are provided in later section.

\subsubsection{Joint Model}

The independent model in Section 2.2.1 is easy to implement, but it fails to consider possible correlations among variables. For example, for either incidence or mortality data, we may expect correlations between males and females within each age group, since people in the same household may share similar diet habit. Moreover, there may be correlations between incidence and mortality data for people in the same category. Building a model which is able to incorporate such kinds of correlations may help us get better estimation and stronger inferential ability. In Schmaltz and Sun (2010) and Schmaltz (2012), there are two models which can address the issue. One is the MarCAR model, which assumes all variables marginally follow regular CAR models. The other one is the ConCAR model, which assumes each variable conditionally follows a regular CAR model given the remaining variables. Both models include parameters accounting for correlations among variables. Before we introduce the models, it is useful to rewrite the independent CAR model in matrix notation. We use the same notations as in Section 2.2.1 for $\boldsymbol{y}, \boldsymbol{\eta}$ and etc. Let $\mathbf{1}_{m}$ be a length $m$ vector with all ones, $\boldsymbol{s}=\left(s_{1}, \ldots, s_{T}\right)^{\prime}$ and $\boldsymbol{\epsilon}_{i}$ is the lack-of-fit error corresponding to $\boldsymbol{y}_{i}$. Then (2.13) can be written as

$$
\boldsymbol{\eta}_{i}=\alpha_{i} \mathbf{1}_{J_{i} T}+\beta_{i} \boldsymbol{s} \otimes \mathbf{1}_{J_{i}}+\mathbf{1}_{T} \otimes \boldsymbol{u}_{i}+\boldsymbol{\epsilon}_{i} .
$$


The prior distributions for $\alpha_{i}, \beta_{i}, \epsilon_{i t j}$ are the same as in (2.14) and (2.16). If we write $\boldsymbol{u}=\left(\boldsymbol{u}_{1}, \ldots, \boldsymbol{u}_{I}\right)^{\prime}$, then the prior for the spatial effect $\boldsymbol{u}$ will be

$$
\boldsymbol{u} \mid \rho_{1}, \ldots, \rho_{I}, \delta_{1}, \ldots, \delta_{I} \sim N_{J_{+}}(\mathbf{0}, \boldsymbol{\Sigma})
$$

where

$$
\boldsymbol{\Sigma}=\boldsymbol{\Delta}^{\frac{1}{2}}\left(\begin{array}{cccc}
\boldsymbol{B}_{1}^{-1} & \mathbf{0} & \cdots & \mathbf{0} \\
\mathbf{0} & \boldsymbol{B}_{2}^{-1} & \cdots & \vdots \\
\vdots & \vdots & \ddots & \mathbf{0} \\
\mathbf{0} & \cdots & \mathbf{0} & \boldsymbol{B}_{p}^{-1}
\end{array}\right) \boldsymbol{\Delta}^{\frac{1}{2}}
$$

and $\boldsymbol{\Delta}=\operatorname{diag}\left(\delta_{1} \boldsymbol{I}_{J_{1}}, \ldots, \delta_{I} \boldsymbol{I}_{J_{I}}\right)$. Inverting $\boldsymbol{\Sigma}$ we can have the precision matrix

$$
\boldsymbol{\Omega}=\boldsymbol{\Delta}^{-\frac{1}{2}}\left(\begin{array}{cccc}
\boldsymbol{B}_{1} & \mathbf{0} & \cdots & \mathbf{0} \\
\mathbf{0} & \boldsymbol{B}_{2} & \cdots & \vdots \\
\vdots & \vdots & \ddots & 0 \\
0 & \cdots & 0 & \boldsymbol{B}_{p}
\end{array}\right) \boldsymbol{\Delta}^{-\frac{1}{2}}
$$

The key idea for the MarCAR and ConCAR model is to introduce the variable level correlations by filling the off diagonal blocks which account for different spatial correlation among variables.

\section{Overlapping matrices.}

For variables defined on the same field with different region partitions, the spatial correlations among those variables can be expressed through the way they are overlapping with each other. Suppose we have two variables $i_{1}$ and $i_{2}$ with $J_{i_{1}}$ and $J_{i_{2}}$ 
number of regions respectively. Let $\boldsymbol{\Psi}_{i_{1}, i_{2}}$ be a size $J_{i_{1}} \times J_{i_{2}}$ matrix to indicate how each region in variable $i_{1}$ is overlapped with those regions in variable $i_{2}$. To be specific, let

$$
\boldsymbol{\Psi}_{i_{1}, i_{2}}=\left(\psi_{i_{1}, i_{2} \mid j_{1}, j_{2}}\right)_{J_{i_{1}} \times J_{i_{2}}},
$$

where $\psi_{i_{1}, i_{2} \mid j_{1}, j_{2}}$ describes the correlation between region $j_{1}$ of variable $i_{1}$ and region $j_{2}$ of variable $i_{2}$. If these two regions $j_{1}$ and $j_{2}$ are not overlapped, then $\psi_{i_{1}, i_{2} \mid j_{1}, j_{2}}=$ 0 . But if they are overlapped, $\psi_{i_{1}, i_{2} \mid j_{1}, j_{2}}$ can be constructed in a number of ways. Schmaltz (2012) discussed several options based on indicators of overlapping regions (indicator similarity), numbers of overlapped atoms (atom similarity) and the area of overlapped regions (area similarity). We will list those optional above together with a new population based overlapping matrix.

\section{Indicator Similarity}

Analogous to the adjacency matrix in CAR models, let $\boldsymbol{\Psi}_{i_{1}, i_{2}}$ be the overlapping matrix to indicate if two regions from two different variables are overlapped or not. Then we have

$$
\psi_{i_{1}, i_{2} \mid j_{1}, j_{2}}=1
$$

when the two regions are overlapped.

\section{Atom Similarity}

Since the regions are created by grouping different counties, the overlapped regions must contain some common counties. The number of counties overlapped may indicate how strongly the variables are correlated. To be specific, let $n_{i_{1} \mid j_{1}}$ be the number of counties in region $j_{1}$ of variable $i_{1}, n_{i_{2} \mid j_{2}}$ be the number of counties in region $j_{2}$ of variable $i_{2}$, and $n_{i_{1}, i_{2} \mid j_{1}, j_{2}}$ be the number of overlapped 
counties between region $j_{1}$ of variable $i_{1}$ and region $j_{2}$ of variable $i_{2}$, then

$$
\psi_{i_{1}, i_{2} \mid j_{1}, j_{2}}=\frac{n_{i_{1}, i_{2} \mid j_{1}, j_{2}}}{\sqrt{n_{i_{1} \mid j_{1}}} \sqrt{n_{i_{2} \mid j_{2}}}} .
$$

\section{Area Similarity}

Similar with the atom similarity, but rather than using the number of overlapped counties to indicate the strength of correlation, we can instead use area. Let $a_{i_{1} \mid j_{1}}$ be the area of region $j_{1}$ of variable $i_{1}, a_{i_{2} \mid j_{2}}$ be the area of region $j_{2}$ of variable $i_{2}$, and $a_{i_{1}, i_{2} \mid j_{1}, j_{2}}$ be the area of the intersection of region $j_{1}$ of variable $i_{1}$ and region $j_{2}$ of variable $i_{2}$, then

$$
\psi_{i_{1}, i_{2} \mid j_{1}, j_{2}}=\frac{a_{i_{1}, i_{2} \mid j_{1}, j_{2}}}{\sqrt{a_{i_{1} \mid j_{1}}} \sqrt{a_{i_{2} \mid j_{2}}}} .
$$

\section{Population Similarity}

For cancer incidence and mortality data, creating a similarity measure using population sizes makes more sense than on geography alone. Let $p_{i_{1} \mid j_{1}}$ be the population size of region $j_{1}$ of variable $i_{1}, p_{i_{2} \mid j_{2}}$ be the population size of region $j_{2}$ of variable $i_{2}$. For different variables, the population sizes are not necessary the same on the overlapped part. Therefore, let $p_{i_{1}\left|j_{1}, i_{2}\right| j_{2}}$ and $p_{i_{2}\left|j_{2}, i_{1}\right| j_{1}}$ be the population sizes on the overlapped part for variables $i_{1}$ and $i_{2}$, then

$$
\psi_{i_{1}, i_{2} \mid j_{1}, j_{2}}=\frac{p_{i_{1}\left|j_{1}, i_{2}\right| j_{2}}+p_{i_{2}\left|j_{2}, i_{1}\right| j_{1}}}{p_{i_{1} \mid j_{1}}+p_{i_{2} \mid j_{2}}}
$$

The construction of $\psi_{i_{1}, i_{2} \mid j_{1}, j_{2}}$ under this population similarity may not be unique. 
For example, it may also be reasonable to use

$$
\psi_{i_{1}, i_{2} \mid j_{1}, j_{2}}=\frac{p_{i_{1}\left|j_{1}, i_{2}\right| j_{2}} * p_{i_{2}\left|j_{2}, i_{1}\right| j_{1}}}{p_{i_{1} \mid j_{1}} * p_{i_{2} \mid j_{2}}} .
$$

The denominators in atom, area and population similarities are used to scale the quantity to match the range in a typical adjacency matrix in CAR model settings. All the entries of the matrices range from 0 to 1, with 0 indicating non-overlap. Except for the indicator similarity, a larger value of $\psi_{i_{1}, i_{2} \mid j_{1}, j_{2}}$ indicate a stronger prior belief of spatial correlation. When two regions are fully overlapped, $\psi_{i_{1}, i_{2} \mid j_{1}, j_{2}}=1$. In that sense, the spatial correlations among neighbors within one variable constructed from adjacency matrix will comparable to the correlations among overlapped regions across variables. Note that others choices of overlapping matrices can be made based on different applications and users' flavors.

\section{MarCAR and ConCAR.}

The construction of overlapping matrices enables us to fill the off diagonal blocks of the covariance matrix $\boldsymbol{\Sigma}$ or precision matrix $\Omega$ and introduce marginal or conditional cross correlations among variables. Let

$$
\boldsymbol{z}=\boldsymbol{\Delta}^{-\frac{1}{2}} \boldsymbol{A}^{\prime} \boldsymbol{u}
$$

be the standardizing transformation of the spatial effect $\boldsymbol{u}$ in (2.24), where $\boldsymbol{A}=$ $\operatorname{diag}\left(\boldsymbol{A}_{1}, \ldots, \boldsymbol{A}_{I}\right)$ and $\boldsymbol{B}_{i}=\boldsymbol{A}_{i} \boldsymbol{A}_{i}^{\prime}, i=1, \ldots, I$. The choice of square root matrices $\boldsymbol{A}_{i}$ is not unique. The Cholesky decomposition was used in our analysis. Then this standardization (2.26) gives $\boldsymbol{z}$ a $J_{+}$(total number of regions for all variables) dimensional 
standard multivariate normal distribution when $\boldsymbol{u}$ is distributed as in (2.24). Such transformation removes the spatial correlation within each variable and enable us to focus on the between-variable correlations only. Let $\boldsymbol{M}=\operatorname{diag}\left(\boldsymbol{I}_{J_{1}}, \boldsymbol{I}_{J_{2}}, \ldots, \boldsymbol{I}_{J_{I}}\right)$ be the identity covariance or precision matrix of $\boldsymbol{z}$ partitioned respect to each $\boldsymbol{z}_{i}$. Its off diagonal blocks can be filled with the overlapping matrices to introduce correlations among all $\boldsymbol{z}_{i}$ s. Thus cross correlations for $\boldsymbol{u}$ can then be introduced by transferring $\boldsymbol{z}$ back to $\boldsymbol{u}$ :

$$
\boldsymbol{u}=\boldsymbol{\Delta}^{\frac{1}{2}}\left(\boldsymbol{A}^{\prime}\right)^{-1} \boldsymbol{z}
$$

This idea is similar to linear coregionalization. However, the idea for filling off diagonal blocks with overlapping matrices, which are constructed based on outside information, is unique for the misaligned problem. With the off diagonal blocks of $\boldsymbol{M}$ filled by overlapping matrices, we construct a new matrix $\boldsymbol{M}_{\boldsymbol{z}}$

$$
\boldsymbol{M}_{\boldsymbol{z}}=\left(\begin{array}{cccc}
\boldsymbol{I}_{J_{1}} & \boldsymbol{\Phi}_{12} & \ldots & \boldsymbol{\Phi}_{1 I} \\
\boldsymbol{\Phi}_{12}^{\prime} & \boldsymbol{I}_{J_{2}} & \ldots & \vdots \\
\vdots & \vdots & \ddots & \boldsymbol{\Phi}_{I-1, I} \\
\boldsymbol{\Phi}_{1, I}^{\prime} & \cdots & \boldsymbol{\Phi}_{I-1, I}^{\prime} & \boldsymbol{I}_{J_{I}}
\end{array}\right)
$$

where $\boldsymbol{\Phi}_{i_{1}, i_{2}}=\gamma_{i_{1} i_{2}} \boldsymbol{\Psi}_{i_{1}, i_{2}}$. The effect of the overlapping matrix $\boldsymbol{\Psi}_{i_{1}, i_{2}}$ is controlled by $\gamma_{i_{1} i_{2}}$, which we denote as the cross correlation parameter. Then the MarCAR model is obtained by treating $\boldsymbol{M}_{\boldsymbol{z}}$ as the covariance matrix of $\boldsymbol{z}$ and the distribution of $\boldsymbol{u}$ is:

$$
\boldsymbol{u} \mid \rho_{1}, \ldots, \rho_{I}, \gamma_{12}, \ldots, \gamma_{I-1, I}, \delta_{1}, \ldots, \delta_{I} \sim N_{J_{+}}\left(\mathbf{0}, \boldsymbol{\Sigma}_{\boldsymbol{u}}\right)
$$


where

$$
\begin{aligned}
\boldsymbol{\Sigma}_{\boldsymbol{u}} & =\boldsymbol{\Delta}^{\frac{1}{2}}\left(\boldsymbol{A}^{-1}\right)^{\prime} \boldsymbol{M}_{\boldsymbol{z}} \boldsymbol{A}^{-1} \boldsymbol{\Delta}^{\frac{1}{2}} \\
& =\left(\begin{array}{cccc}
\delta_{1} \boldsymbol{B}_{1}^{-1} & \gamma_{12} \sqrt{\delta_{1} \delta_{2}}\left(\boldsymbol{A}_{1}^{-1}\right)^{\prime} \boldsymbol{\Psi}_{12} \boldsymbol{A}_{2}^{-1} & \ldots & \gamma_{1 I} \sqrt{\delta_{1} \delta_{I}}\left(\boldsymbol{A}_{1}^{-1}\right)^{\prime} \boldsymbol{\Psi}_{1 I} \boldsymbol{A}_{I}^{-1} \\
\delta_{2} \boldsymbol{B}_{2}^{-1} & \cdots & \vdots \\
& & \ddots & \gamma_{I-1, I} \sqrt{\delta_{I-1} \delta_{I}}\left(\boldsymbol{A}_{I-1}^{-1}\right)^{\prime} \boldsymbol{\Psi}_{I-1, I} \boldsymbol{A}_{I}^{-1} \\
\star & & \delta_{I} \boldsymbol{B}_{I}^{-1}
\end{array}\right) .
\end{aligned}
$$

Here we omitted the lower triangular part for space limitation. This model is called Marginal CAR (MarCAR) since the marginal distribution of $\boldsymbol{u}_{i}$ has a CAR prior (2.3):

$$
\boldsymbol{u}_{i} \mid \delta_{i}, \boldsymbol{B}_{i} \sim N_{J_{i}}\left(\mathbf{0}, \delta_{i} \boldsymbol{B}_{i}^{-1}\right)
$$

On the other hand, the ConCAR model is obtained by treating $\boldsymbol{M}_{\boldsymbol{z}}$ as the precision matrix of $\boldsymbol{z}$ and the distribution of $\boldsymbol{u}$ is:

$$
\boldsymbol{u} \mid \rho_{1}, \ldots, \rho_{I}, \gamma_{12}, \ldots, \gamma_{I-1, I}, \delta_{1}, \ldots, \delta_{I} \sim N_{J_{+}}\left(\mathbf{0}, \Omega_{\boldsymbol{u}}^{-1}\right)
$$


where

$$
\begin{aligned}
\Omega_{\boldsymbol{u}} & =\boldsymbol{\Delta}^{-\frac{1}{2}} \boldsymbol{A} \boldsymbol{M}_{\boldsymbol{z}} \boldsymbol{A}^{\prime} \boldsymbol{\Delta}^{-\frac{1}{2}} \\
& =\left(\begin{array}{cccc}
\delta_{1}^{-1} \boldsymbol{B}_{1} & \gamma_{12}\left(\delta_{1} \delta_{2}\right)^{-\frac{1}{2}} \boldsymbol{A}_{1} \boldsymbol{\Psi}_{12} \boldsymbol{A}_{2}^{\prime} & \cdots & \gamma_{1 I}\left(\delta_{1} \delta_{I}\right)^{-\frac{1}{2}} \boldsymbol{A}_{1} \boldsymbol{\Psi}_{1 I} \boldsymbol{A}_{I}^{\prime} \\
& \delta_{2}^{-1} \boldsymbol{B}_{2} & \cdots & \vdots \\
& & \ddots & \gamma_{I-1, I}\left(\delta_{I-1} \delta_{I}\right)^{-\frac{1}{2}} \boldsymbol{A}_{I-1} \boldsymbol{\Psi}_{I-1, I} \boldsymbol{A}_{I}^{\prime} \\
\star & & & \delta_{I}^{-1} \boldsymbol{B}_{I}
\end{array}\right) .
\end{aligned}
$$

In this case, the conditional distribution of $\boldsymbol{u}_{i}$ given all the other variables is a regular CAR model (2.3):

$$
\boldsymbol{u}_{i} \mid \boldsymbol{u}_{1}, \ldots, \boldsymbol{u}_{i-1}, \boldsymbol{u}_{i+1}, \ldots, \boldsymbol{u}_{I} \sim N_{J_{i}}\left(\mathbf{0}, \delta_{i} \boldsymbol{B}_{i}^{-1}\right),
$$

in which sense it is named as Conditional CAR (ConCAR) model.

The general idea behind the MarCAR or ConCAR model is that we want to retain the CAR prior (2.3) for each variable when those variables are independent of each other. That is desirable since we would simply use the CAR prior if we only have a single variable to work on. When given more variables, we want to enable correlations among the variables while keeping the CAR prior for each variable. The key process of introducing cross correlations is the build of overlapping matrices, which is based on other information. This could help the model fitting when the data itself is not rich enough to show such correlations. 


\section{Interpretation of cross variance parameter $\gamma_{i_{1} i_{2}} \mathbf{s}$.}

Borrowing the concept from linear coregionalization, from the transformation (2.27) we can view variable $\boldsymbol{z}$ as our underlying process which follows a multivariate normal distribution with mean zero and covariance or precision matrix equal to $\boldsymbol{M}_{\boldsymbol{z}}(2.28)$, depending on if we are talking about MarCAR (2.29) model or ConCAR model (2.31).

\section{Identical regions.}

In the case of identical regions for all variables, all the regions are perfectly aligned so that the overlapping matrices $\boldsymbol{\Psi}_{i_{1}, i_{2}}=\boldsymbol{I}_{J_{1}}$. Notice that in this case, we have $J_{1}=J_{2}=\cdots=J_{I}$. Thus we can write

$$
M_{\boldsymbol{z}}=\boldsymbol{\Lambda} \otimes \boldsymbol{I}_{J_{1}},
$$

where

$$
\boldsymbol{\Lambda}=\left(\begin{array}{cccc}
1 & \gamma_{12} & \cdots & \gamma_{1 I} \\
\gamma_{12} & 1 & \cdots & \vdots \\
\vdots & \vdots & \ddots & \gamma_{I-1, I} \\
\gamma_{1, I} & \cdots & \gamma_{I-1, I} & 1
\end{array}\right)
$$

For MarCAR model, $\boldsymbol{\Lambda}$ can be interpreted as cross correlation matrix and $\gamma_{i_{1} i_{2}}$ is the correlation parameter between $\boldsymbol{z}_{i_{1}}$ and $\boldsymbol{z}_{i_{2}}$. For ConCAR model, $\boldsymbol{\Lambda}$ will be the cross precision matrix which actually gives us the $\operatorname{MCAR}\left(\rho_{1}, \ldots, \rho_{I}, \boldsymbol{\Lambda}\right)$ model (2.8). So we can interpret $-\gamma_{i_{1} i_{2}}$ as the partial correlation parameter between $\boldsymbol{z}_{i_{1}}$ and $\boldsymbol{z}_{i_{2}}$ conditional on the rest $\boldsymbol{z}_{i}$ s. Notice the negative sign before $\gamma_{i_{1} i_{2}}$, which gives us positive partial correlation when $\gamma_{i_{1} i_{2}}<0$. Details for interpreting precision matrix in terms of partial correlation can be found in 
Lauritzen (1996).

2. Misaligned regions.

When the regions are misaligned like in our application, we can not separate the covariance matrix in terms of cross variable part and within variable part anymore. Our interpretation can only be at the level

$$
\boldsymbol{M}_{\boldsymbol{z}}=\left(\begin{array}{cccc}
\boldsymbol{I}_{J_{1}} & \gamma_{12} \boldsymbol{\Psi}_{12} & \ldots & \gamma_{1 I} \boldsymbol{\Psi}_{1 I} \\
\gamma_{12} \boldsymbol{\Psi}_{12}^{\prime} & \boldsymbol{I}_{J_{2}} & \ldots & \vdots \\
\vdots & \vdots & \ddots & \gamma_{I-1, I} \boldsymbol{\Psi}_{I-1, I} \\
\gamma_{1 I} \boldsymbol{\Psi}_{1, I}^{\prime} & \ldots & \gamma_{I-1, I} \boldsymbol{\Psi}_{I-1, I}^{\prime} & \boldsymbol{I}_{J_{I}}
\end{array}\right)
$$

When $\boldsymbol{z}_{i_{1}}$ and $\boldsymbol{z}_{i_{2}}$ are two vectors of different length, there is no former definition for covariance matrix between them, since a covariance matrix need to be symmetric. However, we can view $\gamma_{i_{1} i_{2}}$ as some general correlation or partial correlation parameter used to model the correlation among variables. When $\gamma_{i_{1} i_{2}}$ is close to zero, we may conclude $\boldsymbol{z}_{i_{1}}$ and $\boldsymbol{z}_{i_{2}}$ be independent or conditionally independent. On the other hand, if $\gamma_{i_{1} i_{2}}$ is far away from zero, then it may indicate possible strong correlation or partial correlation in the context of MarCAR or ConCAR model.

\section{Positive definiteness conditions for $\Sigma_{u}$ and $\Omega_{u}$.}

For both the MarCAR and ConCAR models, the covariance matrix $\boldsymbol{\Sigma}_{\boldsymbol{u}}$ or precision matrix $\boldsymbol{\Omega}_{u}$ involves many parameters, i.e., $\boldsymbol{\rho}=\left(\rho_{1}, \ldots, \rho_{I}\right)$ and $\boldsymbol{\gamma}=\left(\gamma_{12}, \ldots, \gamma_{I-1, I}\right)$. Restrictions are needed in order to have a valid covariance or precision matrix (Schmaltz, 
2012). To be specific, $\boldsymbol{\rho}$ is restricted the same as the CAR models in the independent model specifications. Conditions for positive-definiteness can be obtained for the bivariate case, but their derivation quickly becomes tedious as the number of variables increases. For the bivariate cases, $\gamma_{12}$ is the only cross variable correlation parameter and should be restricted as

$$
\left|\gamma_{12}\right|<\sqrt{\lambda_{12, J_{2}}^{-1}},
$$

where $\lambda_{12, J_{2}}$ is the largest eigenvalue of $\left(\boldsymbol{\Psi}_{12}\right)^{\prime} \boldsymbol{\Psi}_{12}$. Note that the dimensions of $\boldsymbol{\Psi}_{12}$ are $J_{1} \times J_{2}$. When we have more than two variables, no explicit range for $\gamma$ can be obtained. However, the inequality (2.36) is a necessary condition which can be applied to each element in $\gamma$ accordingly. Please see Appendix A for related proofs.

Constraints on $\gamma$ in multivariate case (more than two) can be solved numerically during our MCMC. Adaptive Rejection Metropolis Sampling (ARMS) (Gilks et al., 1995) can be used to sample $\boldsymbol{\gamma}$. We can first use (2.36) to get the initial range for $\boldsymbol{\gamma}$, then if non-positive-definiteness in the prior distribution of $\boldsymbol{u}$ is detected during the sampling, we can define the posterior density to be zero. In such case only valid $\gamma$ will be sampled, which ensures us always having positive definite matrices.

\subsection{Model Fitting Results}

In this section, we use the independent, MarCAR and ConCAR models to fit CRC incidence and mortality rates in Missouri with different choices of overlapping matrices. 


\subsubsection{Computation and convergence}

We used Gibbs sampling to simulate the posterior samples for all parameters. Based on the model specification in Section 2.2, the full conditional distributions for all the parameters are listed in Appendix B.1. Note that the full conditional distributions are derived only for the MarCAR model, but can be easily extended to the independent and ConCAR models. The independent model is equivalent to setting $\boldsymbol{\gamma}=\mathbf{0}$. For the ConCAR model, we only need to replace $\boldsymbol{\Sigma}_{\boldsymbol{u}}$ with $\boldsymbol{\Omega}_{\boldsymbol{u}}^{-1}$. The four different overlapping matrices described in Section 2.2.2 were tested for the MarCAR and ConCAR models. For all the Gibbs sampling procedure, we used 20,000 posterior samples after the first 10,000 iterations. To speed up our computation, we wrote all the sampling procedure in $\mathrm{C}++$ and called in Microsoft $\mathrm{R}$ open, which is the same as $\mathrm{R}$ but utilizes Intel ${ }^{\circledR}$ Math Kernel Library for faster computations. On our desktop with i7-2600 CPU @ 3.40GHz, the computation time for MarCAR models is around 42.3 hours, while for ConCAR models the time is around 31.7 hours.

To check the convergences of our Gibbs sampling procedures, we monitored the trace plots and histograms for all the parameters (for $\boldsymbol{\eta}$ and $\boldsymbol{u}$ we only randomly selected ten of them). For the ConCAR model with population similarity overlapping matrix, which was used for analysis in later section, we ran four parallel chains and calculated the estimated scale reduction factor $\hat{R}$ as proposed in Gelman and Rubin (1992). Using coda package in $\mathrm{R}$, the $\hat{R}$ statistics were obtained for all the parameters. The results were shown in Figure 2.3a and Figure 2.3b. The parameters $\boldsymbol{\eta}, \boldsymbol{\alpha}, \boldsymbol{\beta}, \boldsymbol{\rho}$ and $\boldsymbol{u}$ have better convergence as their $\hat{R}_{\mathrm{s}}$ are quite close to 1 , while parameters involved in variance estimation, i.e., $\boldsymbol{\gamma}, \boldsymbol{\sigma}^{2}$ and $\boldsymbol{\delta}$ have slightly higher $\hat{R}$. Note that all point estimates of $\hat{R}$ are less than 1.1. 
(a)

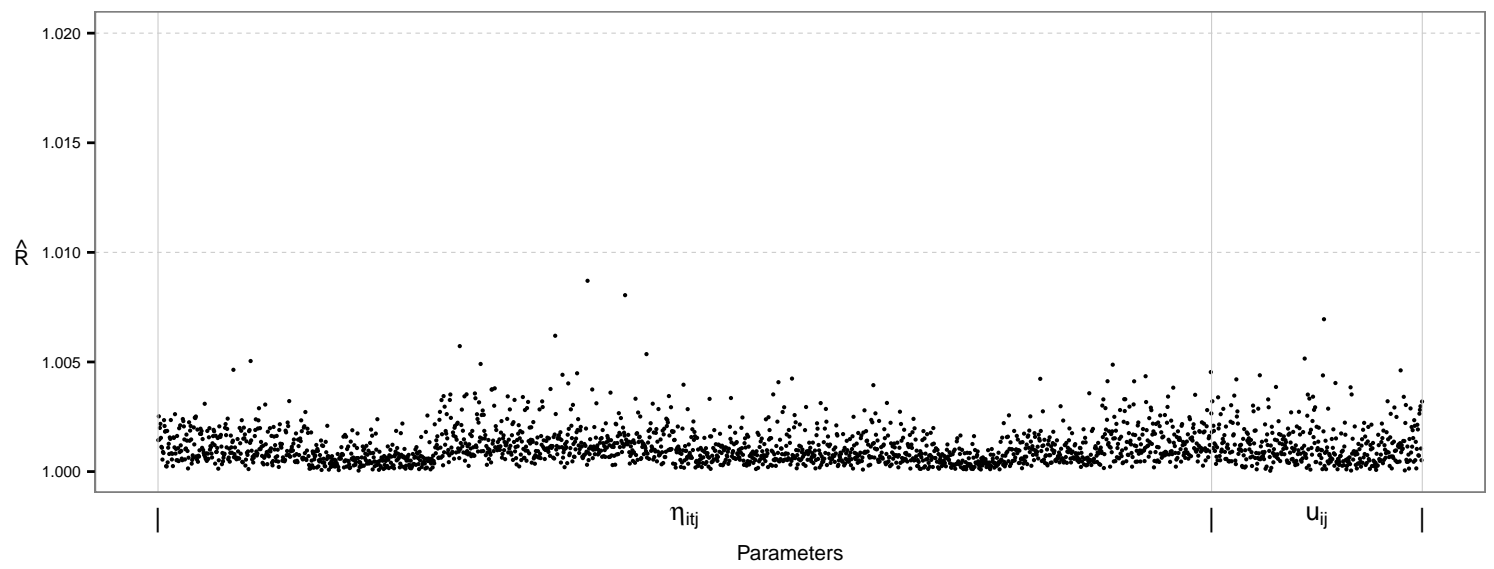

(b)

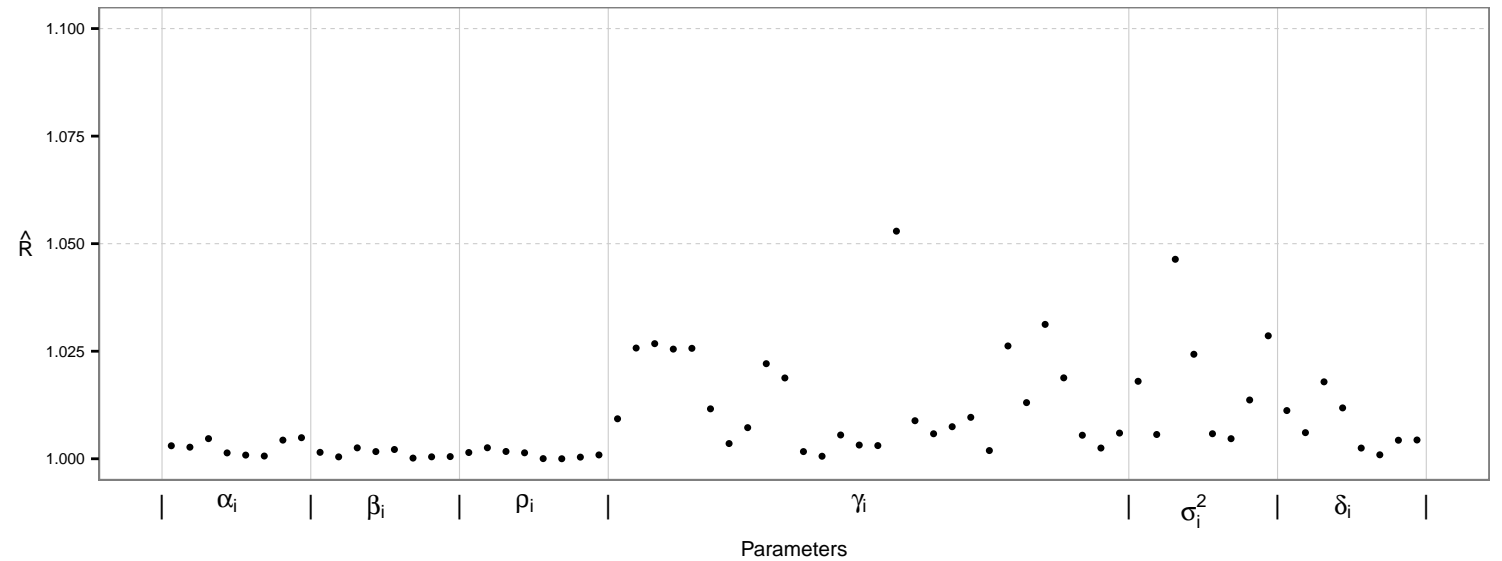

Figure 2.3: Estimated Scale Reduction Factor $\hat{R}$ (a) for $\boldsymbol{\eta}$ and $\boldsymbol{u}$; (b) for $\boldsymbol{\alpha}, \boldsymbol{\beta}, \boldsymbol{\rho}, \boldsymbol{\gamma}, \boldsymbol{\sigma}^{2}, \boldsymbol{\delta}$. Four parallel chains were used for calculation, with each having 20,000 posterior samples after discarding the first 10,000 ones.

\subsubsection{Sensitivity analysis of priors for variance parameters}

A sensitivity analysis was conducted mainly focused on the choice of hyper parameters for the variance parameters $\sigma_{i}^{2}$ and $\delta_{i}$ (or $\omega_{i}$ as the ratio). As in section 5.3.5, we assign $\mathrm{HC}(\zeta)$ distribution on standard deviation parameter $\sigma_{i}$ and the prior of $\delta_{i}$ is specified through the noise-to-signal ratio $\omega_{i}$, which has a $\operatorname{Pareto}(a)$ distribution. In our application, $\zeta=1$ and $a=1$ were used. To check the robustness of those 
two parameters, we reran the ConCAR model with population similarity under six different settings. We choose $\zeta=0.3,1,2,5$ while fixing $a=1$ to investigate the effect of $\zeta$. Later on, we fix $\zeta=1$ and set $a=0.5,2$ to check the effect of $a$. Table 2.2 contains the posterior mean and standard deviations for $\sigma_{i}^{2}$ and $\delta_{i}$ based on 40,000 posterior samples after discarding first 10,000 iterations. When we increase the value of $\zeta$ from 0.3 to 5 , the estimated variances did not change a lot. No clear relationship between the estimates and $\zeta$ was found during our analysis. If we compare the posterior means under $a=1$ and $a=0.5,2$, we can see that the estimates of $\sigma_{i}^{2}$ is generally smaller when $a=0.5$ and larger when $a=2$, while the estimates of $\delta_{i}$ show the opposite reaction. This is expected as $a>1$ puts more weight on structured spatial effect while $a<1$ puts more weight on the unstructured random error. Comparing the potential impacts of choices on $\zeta$ and $a$, the value of $a$ seems to have relatively larger effect on the posterior inference for the variance parameters.

Despite the possible impacts on the variance parameters, the rate estimates are rather robust across all scenarios. If we treat $\zeta=1$ and $a=1$ as the reference model, then the maximum changes in terms of rate estimates are 1.20, 0.82, 4.01, $2.62,0.43,0.46,0.98$ and 1.36 cases per 100,000 people per year for those 8 variables, respectively. 


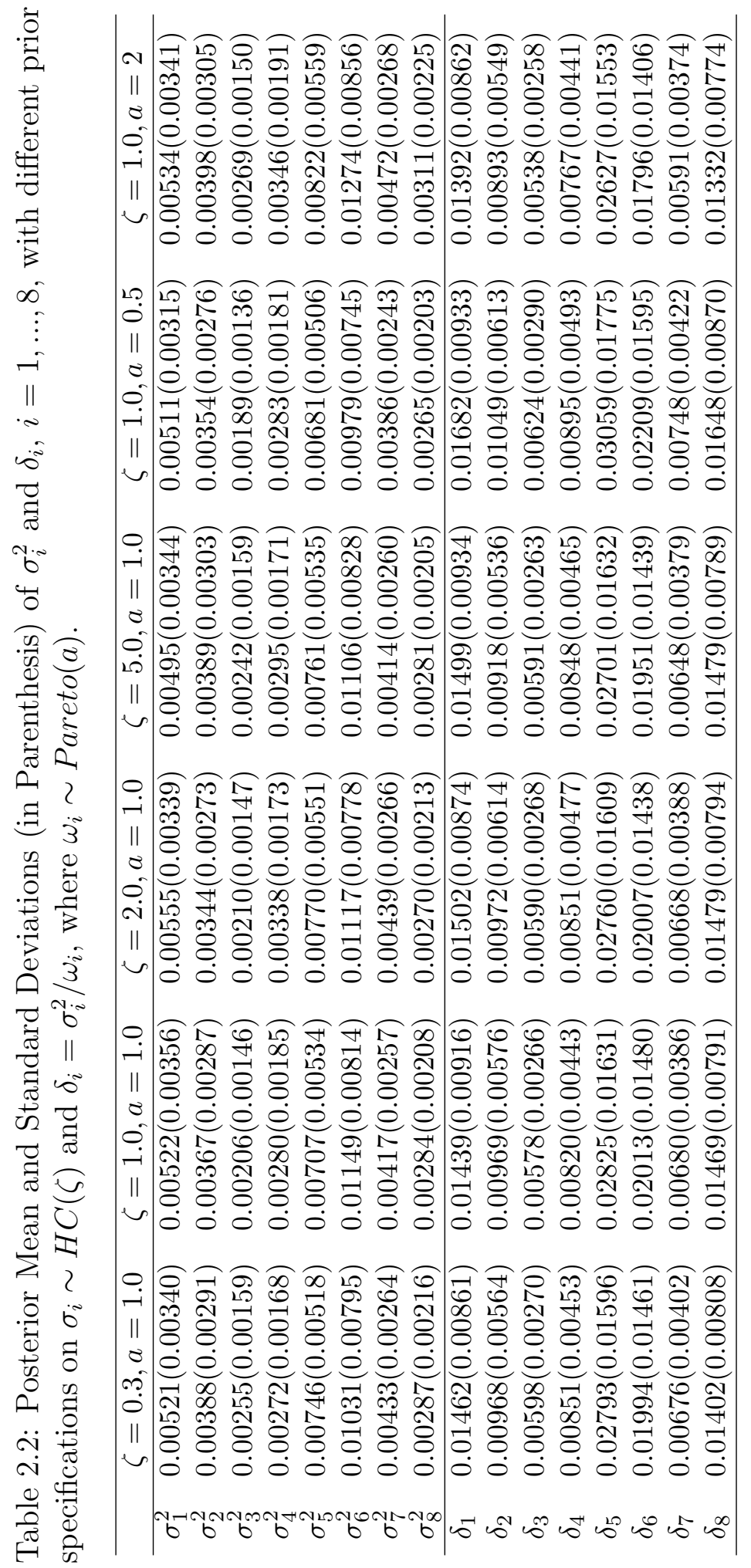




\subsubsection{Model selection}

\section{Comparison among different models.}

We fitted the independent, MarCAR and ConCAR models with different choices of overlapping matrices. Note that we used (2.25) for the population similarity. Based on posterior samples of parameters, we calculated the Deviance Information Criterion (DIC) (Spiegelhalter et al., 2002) and the log-pseudo marginal likelihood (LPML) for model comparison. LPML was calculated as the sum of log conditional predictive ordinate (CPO) (Box, 1980; Chen et al., 2012; Gelfand et al., 1992) for each observation. Table 2.3 shows the DICs and LPMLs, together with the estimated number

Table 2.3: DIC for the Independent, MarCAR and ConCAR models

\begin{tabular}{llrrrr}
\hline Model & Choice of $\boldsymbol{\Psi}$ & Deviance & $p_{D}$ & DIC & LPML \\
\hline Independent & ZERO & 11686.08 & 361.09 & 12047.17 & -6041.85 \\
\hline \multirow{4}{*}{ MarCAR } & Indicator Similarity & 11670.01 & 337.87 & 12007.88 & -6017.47 \\
& Atom Similarity & 11672.55 & 317.22 & 11989.77 & -6006.94 \\
& Area Similarity & 11665.80 & 322.17 & 11987.97 & -6005.30 \\
& Population Similarity & 11672.11 & 313.15 & 11985.26 & -6003.66 \\
\hline \multirow{5}{*}{ ConCAR } & Indicator Similarity & 11682.91 & 331.80 & 12014.71 & -6021.30 \\
& Atom Similarity & 11712.67 & 273.41 & 11986.08 & -6005.09 \\
& Area Similarity & 11703.96 & 281.69 & 11985.65 & -6004.88 \\
& Population Similarity & 11695.23 & 287.35 & 11982.58 & -6003.56 \\
\hline
\end{tabular}

of effective parameters $\left(p_{D}\right)$ and the deviance. Models with smaller DICs are preferred. However, for LPML we prefer larger values. First, we notice that DIC is consistent with LPML in our case. When one model has a smaller DIC, it has a larger LPML. Thus conclusions based on DIC will be the same as looking at LPML. The independent model has the largest DIC, which can be treated as our baseline while all the other models have much smaller DICs. For each type of overlapping 
matrices, the MarCAR and ConCAR models are generally comparable with similar DICs. However, MarCAR models provide better fits with smaller deviances, while ConCAR models have stronger shrinkage effects on the number of parameters (smaller $\left.p_{D}\right)$. The population similarity provides the best fit for both MarCAR and ConCAR models, where they both have the smallest DICs and the almost the same LPML. There is no clear evidence which one is better. But since ConCAR model is faster to fit than MarCAR model, we decided to focus on our analysis on ConCAR model with population similarity.

\section{Simplification of cross variable correlations.}

The ConCAR model is used in our data analysis based on DICs. Even with the use of precision matrix, there is still a large computational burden. The ARMS algorithm is needed for every posterior sample of $\gamma$ and the evaluation of the log-density is expensive. From Section 2.3.3 we know the joint model is much better than the independent model. However, it is not necessary that every pair of variables should be partially correlated given the presence of other variables. To set two variables $i_{1}$ and $i_{2}$ conditionally independent given all the other variables in a ConCAR model, we simply set their corresponding $\gamma_{i_{1} i_{2}}=0$. We used posterior means of $\boldsymbol{\gamma}$ as their estimates. If the estimated $\gamma_{i_{1} i_{2}}$ is close to zero, then the variables $i_{1}$ and $i_{2}$ may be conditional independent, in which case we can set $\gamma_{i_{1} i_{2}}=0$ for a simplified model. In Figure 2.4, the posterior means and $95 \%$ credible intervals for $\gamma$ are list in the format of the matrix $\boldsymbol{\Lambda}$ (2.34). The values are based on 80,000 posterior samples mixed from the four parallel chains during our convergence diagnosis. Although $\boldsymbol{\Lambda}$ can not be interpreted as cross precision matrix, the scales of $\gamma$ can indicate if two variables 


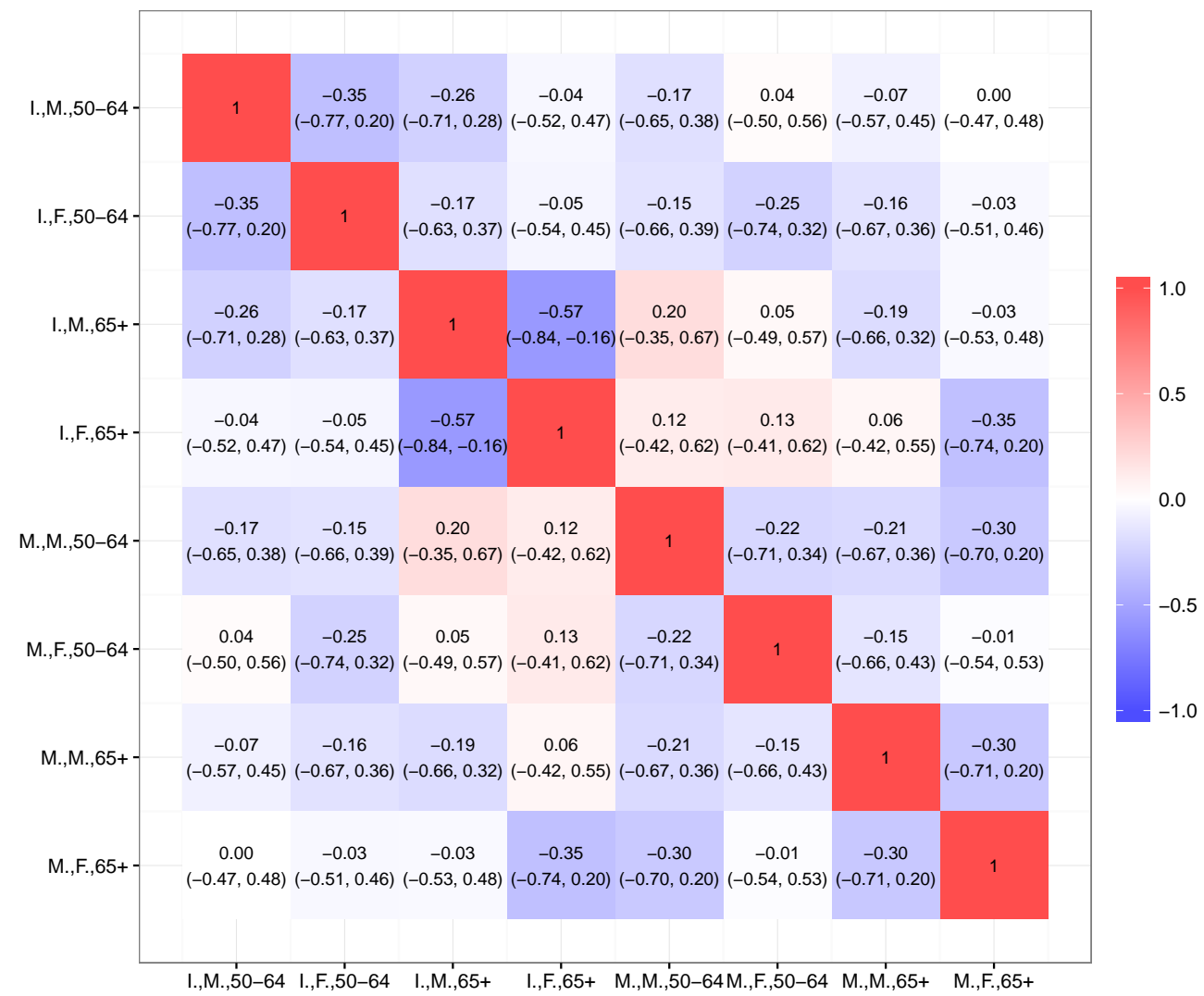

Figure 2.4: Posterior Mean of Cross Variable Precision Matrix for the Full ConCAR Model. This matrix is constructed by posterior means of $\gamma_{i_{1} i_{2}}$ s from the full ConCAR model. The 8 variables are ordered 1 to 8 in the matrix from left to right, or up and down.

should be conditionally independent or not. The further $\gamma_{i_{1} i_{2}}$ is away from zero, the less likely variables $i_{1}$ and $i_{2}$ are conditionally independent. Based on the absolute value of the estimated $\gamma$, we can conclude the following partial relationships:

(i) Males and females within the same age group are partially correlated for both incidence and mortality data $\left(\gamma_{12}=-0.35, \gamma_{34}=-0.57, \gamma_{56}=-0.22, \gamma_{78}=\right.$ $-0.30)$

(ii) Incidence and mortality are partially correlated for people in same age-gender 


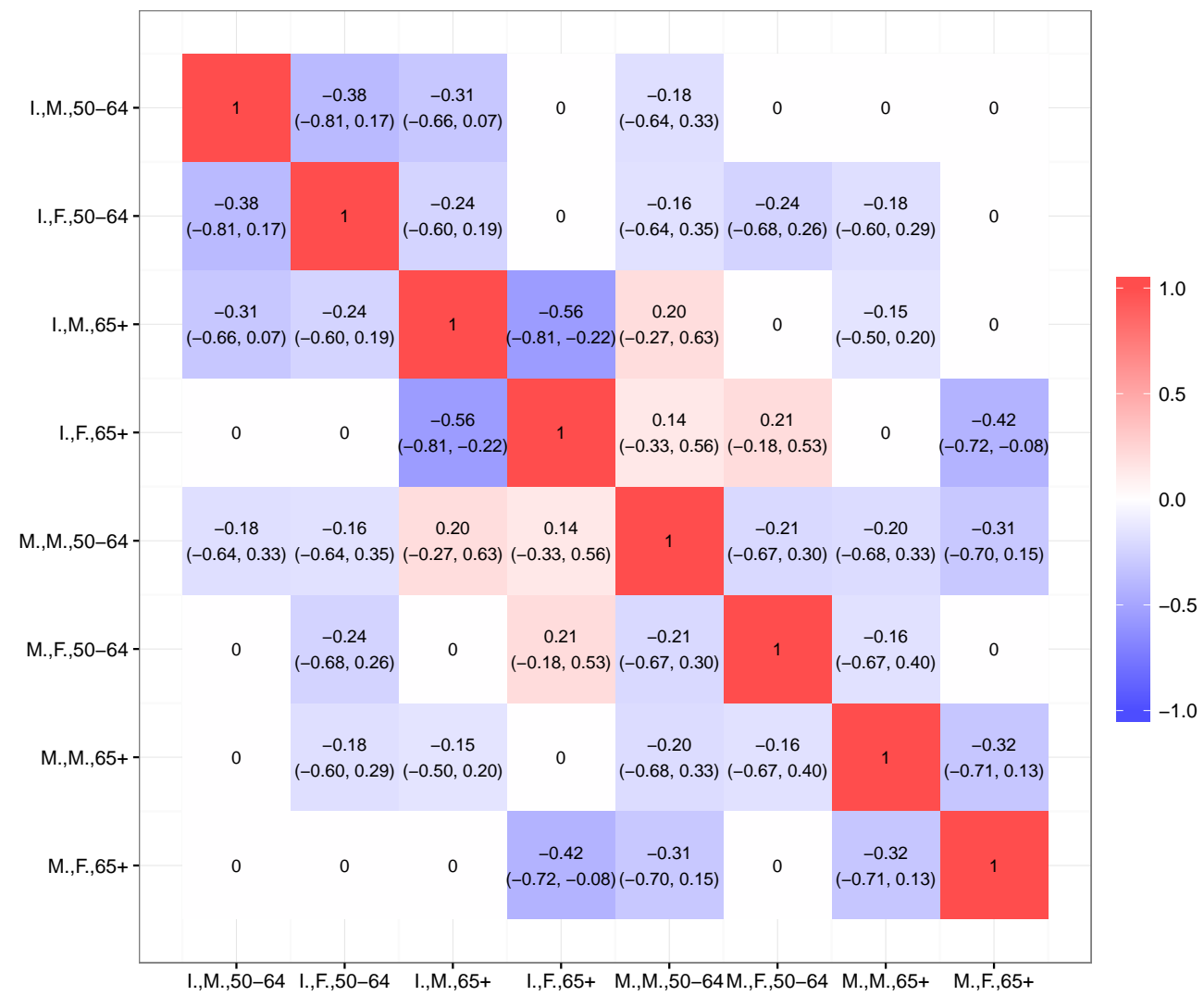

Figure 2.5: Posterior Mean of Cross Variable Precision Matrix for the Simplified ConCAR Model. This matrix is constructed by posterior means of $\gamma_{i_{1} i_{2}}$ s from the Simplified ConCAR model, where $\gamma_{14}, \gamma_{16}, \gamma_{17}, \gamma_{18}, \gamma_{24}, \gamma_{28}, \gamma_{36}, \gamma_{38}, \gamma_{47}, \gamma_{68}$ are set to zeros as shown in the matrix. The 8 variables are ordered 1 to 8 in the matrix from left to right, or up and down.

groups $\left(\gamma_{15}=-0.17, \gamma_{26}=-0.25, \gamma_{37}=-0.19, \gamma_{48}=-0.35\right)$;

(iii) Males aged 50-64 and 65+ are partially correlated for both incidence and mortality data $\left(\gamma_{13}=-0.26, \gamma_{57}=-0.21\right)$, while the correlation is not strong for females $\left(\gamma_{24}=-0.05, \gamma_{38}=-0.01\right)$.

(iv) For mortality data, females aged 65+ are partially correlated with males aged 50-64 $\left(\gamma_{58}=-0.30\right)$, while it is not the case for incidence data $\left(\gamma_{14}=-0.04\right)$. 
(v) The incidence rate for males and females aged 65+ are partially correlated with the mortality rate for those aged 50-64, respectively $\left(\gamma_{35}=0.20, \gamma_{46}=0.13\right)$.

(vi) For both incidence and mortality, females aged 50-64 has partial correlation with males aged $65+\left(\gamma_{23}=-0.21, \gamma_{67}=-0.18\right)$.

(vii) The incidence rate for females aged 50-64 is partially correlated with the mortality rate for males aged $65+\left(\gamma_{27}=-0.16\right)$. The incidence rate for females aged $65+$ is partially correlated with the mortality rate for males aged 50-64 $\left(\gamma_{45}=0.21\right)$.

Findings of (i), (ii) and (iii) are more intuitive than the rest since for the cancergender-age combination, they only vary in one aspect. Findings of (iv), (v), (vi) and (vii) are less straightforward to come up with. However, since the corresponding $\gamma$ elements are relatively large (more than 0.1 in absolute value), we decided to list them here. The cut off value 0.1 was used in our analysis. However, there is no specific definition or guidance of choosing the cut off value. For the rest of unmentioned $\gamma$ elements, their absolute values are all less than 0.1 . Thus we simplify our model by restricting

$$
\gamma_{14}=\gamma_{16}=\gamma_{17}=\gamma_{18}=\gamma_{24}=\gamma_{28}=\gamma_{36}=\gamma_{47}=\gamma_{68}=0
$$

We call this simplified mode as the Simplified ConCAR with population similarity. To check if the Simplified ConCAR model is better than the full ConCAR model, we calculated the DIC and LPML. The Simplified ConCAR has Deviance=11713.07, $p_{D}=265.91, \mathrm{DIC}=11978.98$ and $\mathrm{LMPL}=-6000.856$. Compare with the full ConCAR model in Table 2.3, the simplified ConCAR model has slightly worse fit but reduced 
number of effective parameters and reduced DIC. Based on this result, we used the Simplified ConCAR model for the following analysis. The estimated $\boldsymbol{\gamma}$ for Simplified ConCAR model are listed in Figure 2.5. By setting the $\gamma$ elements in (2.37) to be zero, the scales of the other free $\gamma$ elements were enlarged.

\subsubsection{Numerical analysis}

\section{Parameter estimation}

Table 2.4 summarizes the posterior means, standard deviations and quantiles for $\boldsymbol{\alpha}, \boldsymbol{\beta}, \boldsymbol{\sigma}^{2}, \boldsymbol{\rho} \boldsymbol{\delta}$ and $\boldsymbol{\rho}$ based on posterior samples from the independent and Simplified ConCAR models. The posterior summaries for $\boldsymbol{\alpha}$ and $\boldsymbol{\beta}$ are identical for both models. However, the estimates for the $\boldsymbol{\sigma}^{2}, \boldsymbol{\delta}$ and $\boldsymbol{\rho}$ vary a lot between independent and simplified ConCAR models. The independent model has larger estimates for $\boldsymbol{\sigma}^{2}, \boldsymbol{\delta}$ and $\boldsymbol{\rho}$ compared to the Simplified ConCAR model. It is expected since part of the variation in each independent model will be shared by cross variable correlation when we jointly model all variables.

The posterior densities of the spatial correlation parameter $\boldsymbol{\rho}$ are shown in Figure 2.6. Only the incidence rates for males and females aged $65+$ and the mortality rates for females aged $65+$ indicate strong spatial associations. 
Table 2.4: Posterior Quantities for Independent and Simplified ConCAR Models. The subscripts for all the parameters are consistent with the variable order in Table 2.1. Sd: standard deviation.

\begin{tabular}{|c|c|c|c|c|c|c|c|c|c|c|}
\hline & \multicolumn{5}{|c|}{ Independent Model } & \multicolumn{5}{|c|}{ Simplified ConCAR Model } \\
\hline & Mean & $\mathrm{Sd}$ & $2.5 \%$ & Median & $97.5 \%$ & Mean & $\mathrm{Sd}$ & $2.5 \%$ & Median & $97.5 \%$ \\
\hline$\alpha_{1}$ & -5.8052 & 0.0150 & -5.8342 & -5.8053 & -5.7757 & -5.8112 & 0.0156 & -5.8417 & -5.8112 & -5.7806 \\
\hline$\alpha_{2}$ & -6.1937 & 0.0159 & -6.2250 & -6.1934 & -6.1630 & -6.2031 & 0.0177 & -6.2381 & -6.2031 & -6.1677 \\
\hline$\alpha_{3}$ & -4.6809 & 0.0108 & -4.7019 & -4.6811 & -4.6595 & -4.6754 & 0.0107 & -4.6964 & -4.6755 & -4.6550 \\
\hline$\alpha_{4}$ & -4.9362 & 0.0105 & -4.9563 & -4.9361 & -4.9151 & -4.9306 & 0.0109 & -4.9520 & -4.9306 & -4.9094 \\
\hline$\alpha_{5}$ & -7.0167 & 0.0254 & -7.0672 & -7.0162 & -6.9680 & -7.0189 & 0.0252 & -7.0671 & -7.0193 & -6.9690 \\
\hline$\alpha_{6}$ & -7.4783 & 0.0300 & -7.5386 & -7.4779 & -7.4198 & -7.4807 & 0.0305 & -7.5413 & -7.4807 & -7.4212 \\
\hline$\alpha_{7}$ & -5.5658 & 0.0148 & -5.5952 & -5.5657 & -5.5371 & -5.5612 & 0.0149 & -5.5907 & -5.5610 & -5.5328 \\
\hline$\alpha_{8}$ & -5.7935 & 0.0152 & -5.8232 & -5.7935 & -5.7647 & -5.7965 & 0.0146 & -5.8257 & -5.7965 & -5.7685 \\
\hline$\beta_{1}$ & -0.0994 & 0.0155 & -0.1299 & -0.0995 & -0.0682 & -0.0989 & 0.0153 & -0.1289 & -0.0989 & -0.0689 \\
\hline$\beta_{2}$ & -0.0832 & 0.0171 & -0.1166 & -0.0834 & -0.0488 & -0.0819 & 0.0170 & -0.1150 & -0.0818 & -0.0488 \\
\hline$\beta_{3}$ & -0.1758 & 0.0108 & -0.1970 & -0.1758 & -0.1545 & -0.1763 & 0.0104 & -0.1961 & -0.1766 & -0.1553 \\
\hline$\beta_{4}$ & -0.1613 & 0.0104 & -0.1820 & -0.1611 & -0.1415 & -0.1608 & 0.0101 & -0.1809 & -0.1608 & -0.1409 \\
\hline$\beta_{5}$ & -0.0402 & 0.0269 & -0.0927 & -0.0404 & 0.0126 & -0.0412 & 0.0271 & -0.0937 & -0.0417 & 0.0128 \\
\hline$\beta_{6}$ & -0.0901 & 0.0339 & -0.1563 & -0.0899 & -0.0238 & -0.0889 & 0.0332 & -0.1535 & -0.0891 & -0.0233 \\
\hline$\beta_{7}$ & -0.1277 & 0.0158 & -0.1585 & -0.1276 & -0.0970 & -0.1270 & 0.0160 & -0.1573 & -0.1273 & -0.0952 \\
\hline$\beta_{8}$ & -0.1531 & 0.0150 & -0.1822 & -0.1533 & -0.1236 & -0.1518 & 0.0143 & -0.1796 & -0.1518 & -0.1237 \\
\hline$\sigma_{1}^{2}$ & 0.0069 & 0.0040 & 0.0007 & 0.0064 & 0.0158 & 0.0061 & 0.0036 & 0.0007 & 0.0055 & 0.0143 \\
\hline$\sigma_{2}^{2}$ & 0.0056 & 0.0036 & 0.0011 & 0.0047 & 0.0147 & 0.0037 & 0.0029 & 0.0004 & 0.0029 & 0.0111 \\
\hline$\sigma_{3}^{2}$ & 0.0034 & 0.0021 & 0.0007 & 0.0030 & 0.0084 & 0.0026 & 0.0017 & 0.0002 & 0.0023 & 0.0067 \\
\hline$\sigma_{4}^{2}$ & 0.0038 & 0.0022 & 0.0004 & 0.0035 & 0.0086 & 0.0032 & 0.0018 & 0.0005 & 0.0029 & 0.0075 \\
\hline$\sigma_{5}^{2}$ & 0.0094 & 0.0063 & 0.0012 & 0.0081 & 0.0245 & 0.0075 & 0.0054 & 0.0006 & 0.0065 & 0.0207 \\
\hline$\sigma_{6}^{2}$ & 0.0144 & 0.0097 & 0.0020 & 0.0123 & 0.0379 & 0.0114 & 0.0083 & 0.0010 & 0.0095 & 0.0325 \\
\hline$\sigma_{7}^{2}$ & 0.0055 & 0.0031 & 0.0004 & 0.0052 & 0.0127 & 0.0042 & 0.0025 & 0.0006 & 0.0038 & 0.0101 \\
\hline$\sigma_{8}^{2}$ & 0.0036 & 0.0026 & 0.0003 & 0.0030 & 0.0101 & 0.0026 & 0.0020 & 0.0003 & 0.0021 & 0.0078 \\
\hline$\delta_{1}$ & 0.0484 & 0.0211 & 0.0161 & 0.0454 & 0.0979 & 0.0151 & 0.0097 & 0.0033 & 0.0128 & 0.0408 \\
\hline$\delta_{2}$ & 0.0222 & 0.0137 & 0.0032 & 0.0196 & 0.0561 & 0.0071 & 0.0048 & 0.0015 & 0.0059 & 0.0195 \\
\hline$\delta_{3}$ & 0.0201 & 0.0085 & 0.0076 & 0.0189 & 0.0402 & 0.0 & 0.0028 & 0.0022 & 0.0052 & 0.0128 \\
\hline$\delta_{4}$ & 0.0231 & 0.0098 & 0.0082 & 0.0215 & 0.0459 & 0.0085 & 0.0048 & 0.0025 & 0.0074 & 0.0205 \\
\hline$\delta_{5}$ & 0.0800 & 0.0409 & 0.0260 & 0.0719 & 0.1838 & & 0.0163 & 0.0076 & 0.0233 & 0.0684 \\
\hline$\delta_{6}$ & 0.0431 & 0.0323 & 0.0029 & 0.0361 & 41 & 0. & 0.0159 & 0.0040 & 0.0178 & 0.0633 \\
\hline$\delta_{7}$ & 0.0166 & 0.0088 & 0.0043 & 0.0150 & 0.0383 & 0.0063 & 0.0038 & 0.0016 & 0.0055 & 0.0159 \\
\hline$\delta_{8}$ & 0.0442 & 0.0193 & 0.0169 & 0.0410 & 0.0907 & 0.0142 & 0.0082 & 0.0038 & 0.0125 & 0.0351 \\
\hline$\rho_{1}$ & 0.5504 & 0.2593 & 0.0430 & 0.5803 & 0.9465 & 0.4628 & 0.2602 & 0.0260 & 0.4628 & 0.9236 \\
\hline$\rho_{2}$ & 0.4645 & 0.2730 & 0.0219 & 0.4609 & 0.9429 & 0.3702 & 0.2440 & 0.0160 & 0.3396 & 0.8680 \\
\hline$\rho_{3}$ & 0.7571 & 0.2139 & 0.1682 & 0.8251 & 0.9860 & 0.6406 & 0.2321 & 0.1066 & 0.6869 & 0.9596 \\
\hline$\rho_{4}$ & 0.8524 & 0.1534 & 0.4046 & 0.9029 & 0.9925 & 0.7306 & 0.2013 & 0.2065 & 0.7843 & 0.9705 \\
\hline$\rho_{5}$ & 0.4828 & 0.2636 & 0.0283 & 0.4894 & 0.9374 & 0.3870 & 0.2487 & 0.0167 & 0.3630 & 0.8905 \\
\hline$\rho_{6}$ & 0.4896 & 0.2714 & 0.0280 & 0.4937 & 0.9472 & 0.4859 & 0.2642 & 0.0297 & 0.4925 & 0.9357 \\
\hline$\rho_{7}$ & 0.6085 & 0.2650 & 0.0527 & 0.6602 & 0.9714 & 0.5001 & 0.2599 & 0.0340 & 0.5124 & 0.9355 \\
\hline$\rho_{8}$ & 0.7413 & 0.2120 & 0.1773 & 0.8021 & 0.9834 & 0.5966 & 0.2469 & 0.0684 & 0.6366 & 0.9535 \\
\hline
\end{tabular}




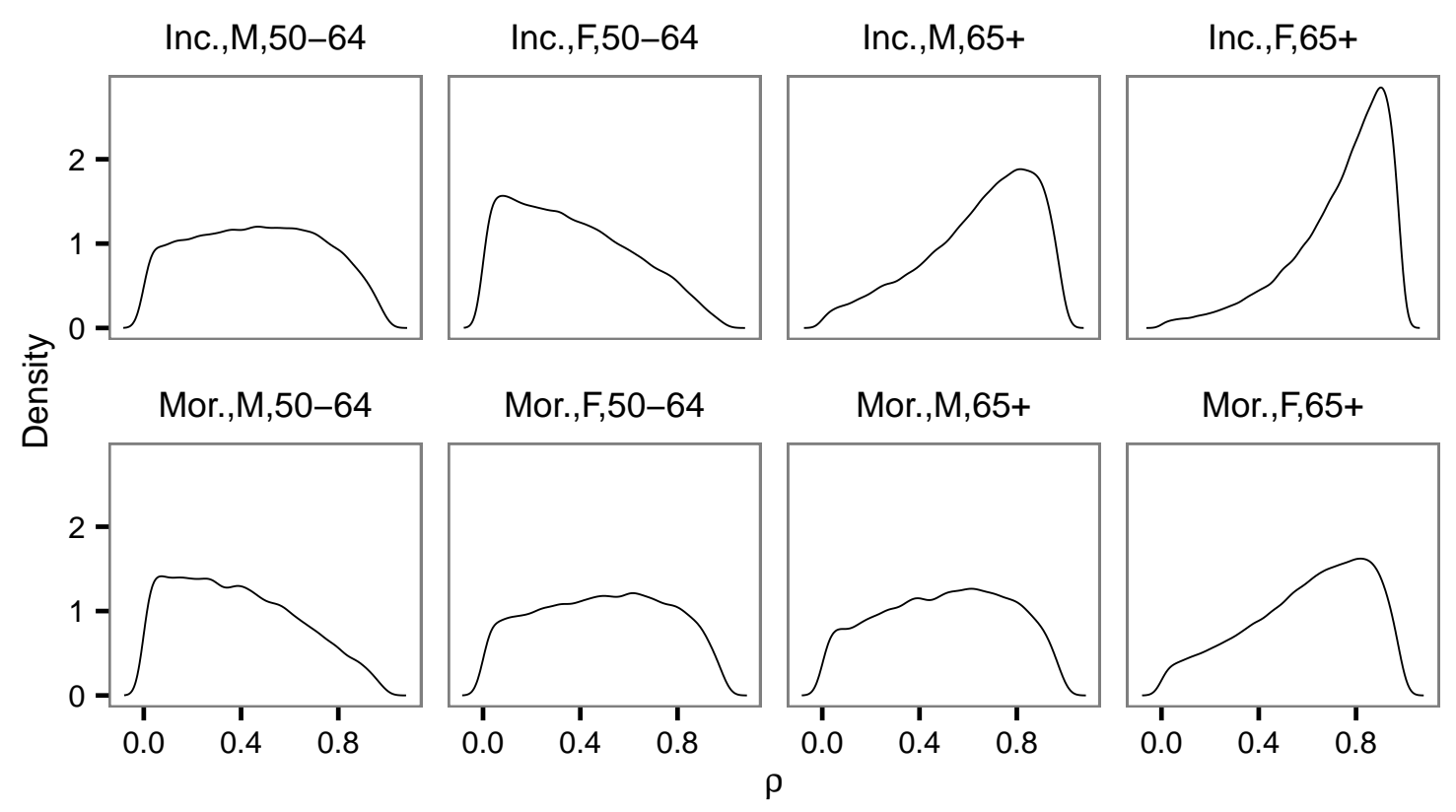

Figure 2.6: Posterior Densities for Spatial Correlation Parameter $\rho_{i}, i=1, \ldots, 8$.

\section{Incidence and mortality rates estimation}

Rates comparison between Independent and ConCAR models. With the use of the ConCAR models, the correlations among our 8 variables have been established in Section 2.3.3. The DIC for the Simplified ConCAR is much smaller than that for the independent model. To investigate the effects of fitting Simplified ConCAR models more closely, we plotted the posterior means and standard deviations of rate estimates in Figure 2.7. Points of different colors stand for different variables. We can see that there is almost no difference between the Independent and Simplified ConCAR models in terms of posterior means of rates, with all points being around the diagonal line. However, the rates from the Simplified ConCAR models have smaller standard deviations than rates from independent models. Averaged over all posterior samples of rates, there is a $15.6 \%$ reduction in standard deviation relative to the 
(a) Posterior Mean

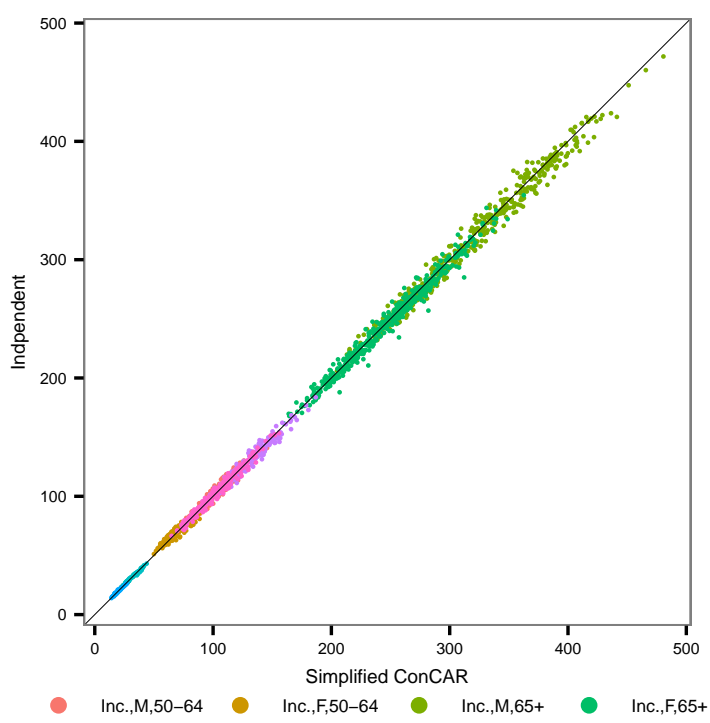

(b) Posterior Standard Deviation

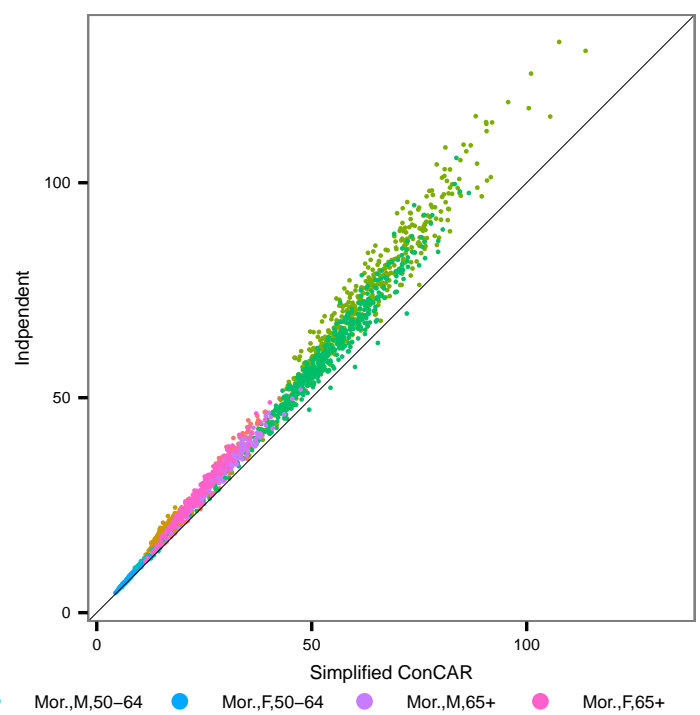

Figure 2.7: Scatterplots of Posterior Means and Standard Deviations of Rates Based on the Independent and Simplified ConCAR Model for 8 Different Variables.

independent model.

Rates Mapping. Based on the posterior samples of the log-rates $\boldsymbol{\eta}$, the posterior samples of rates per 100,000 people per year can be obtained as $\exp (\boldsymbol{\eta}) \times 100000$. The posterior means and standard deviations can be calculated and we used the posterior means as our estimates. We plotted the posterior rate estimates (smoothed rates) in Figure 2.8. Compared with Figure 2.1 which shows the frequentist rate estimates, the spatial smoothing effects are quite obvious with our smoothed rates. Note that the color legend scales are different among panels (a)-(d), and also different from those in Figure 2.1. Red colors stand for higher rates, blue colors stand for lower rates, and white is used for the statewide incidence (mortality) rates for the corresponding age groups. The smoothed rates have narrower ranges than the frequentist rates, which means the spatial effects in our ConCAR model shrank the rates towards their mean values. All panels in Figure 2.8 indicate males have higher rates than females and 
the rates are decreasing over time. In general, the north and-to a lesser extentthe southeast regions of Missouri have higher rates than the others. The smoothed mortality rates for females aged 50-64 appear to be distributed slightly differently than the others, with the rates fairly low across the state except in the Missouri Bootheel (southeast) region.

Statewide rates. Given the estimated rates for each region, the statewide rate can be calculated by incorporating the population size for each region. Figure 2.9 shows the statewide incidence rates on the left and mortality rates on the right. The solid and dashed lines are the smoothed results based on posterior means of statewide rates from the Simplified ConCAR model. The solid and empty points are the statewide rates calculated based on (2.11), which are referred as frequentist rates in our analysis. The two results are consistent with each other. From the plots, we can see people aged $65+$ had higher rates than people aged 50-64, and males had higher rates than females with substantial differences. Also, all rates have declined over time.

\subsection{Discussion}

In this chapter, we analyzed $\mathrm{CRC}$ incidence and mortality rates in Missouri with focus on people aged over 50 years old, with gender, time and location as our covariates. We build upon previous results from the MarCAR and ConCAR models by introducing a new overlapping matrix (Population Similarity). In most epidemiological studies, the population sizes will be more informative to help incorporating correlations among

different variables. In addition, the previous models were extended to the spatio- 


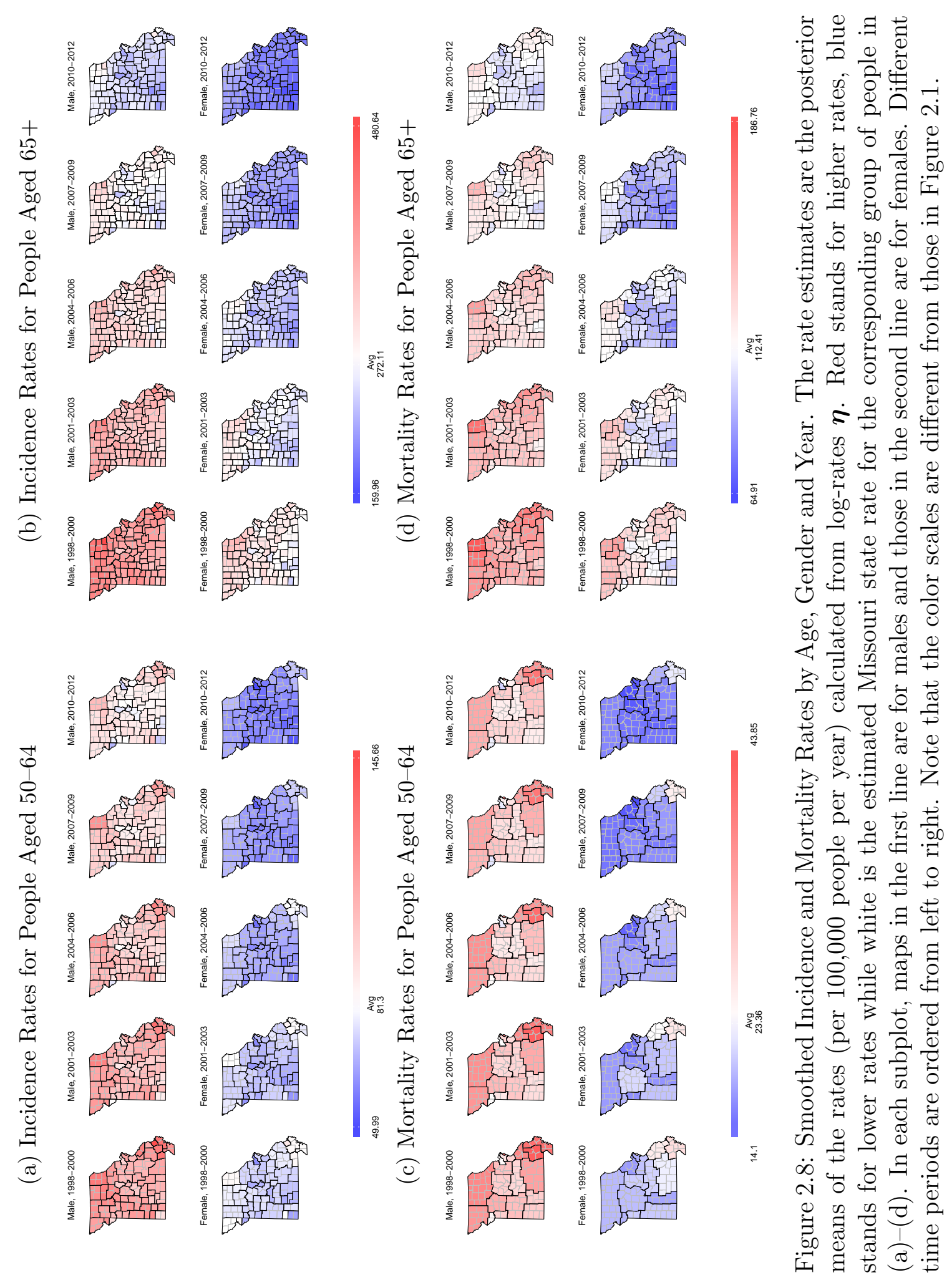


(a) Incidence Rate

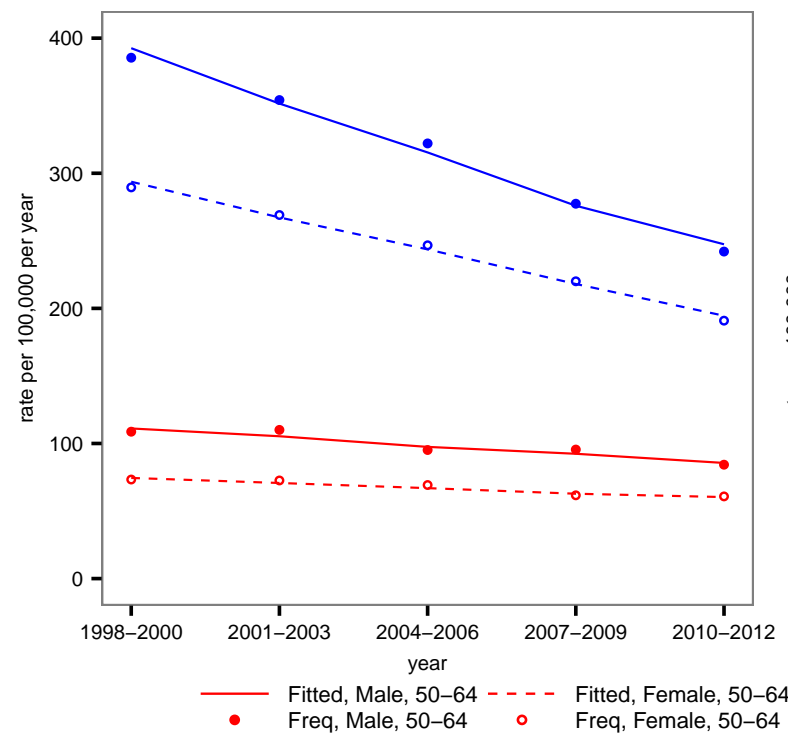

(b) Mortality Rate

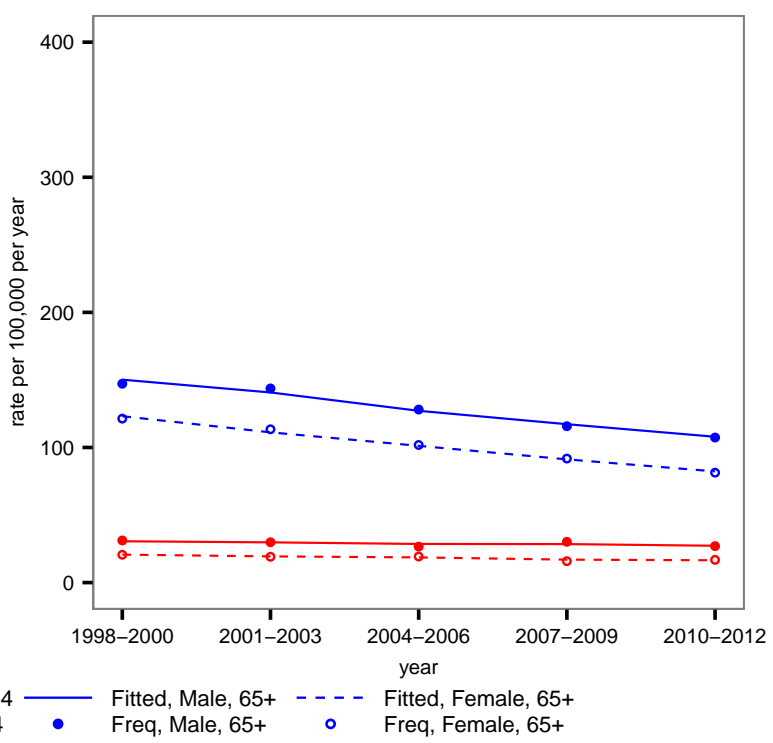

Figure 2.9: Estimated Statewide Incidence and Mortality Rates for Each Year. The points are frequentist rates based on (2.11), while the lines are the smoothed rates from the Simplified ConCAR model.

temporal setting, which allows us to study rate changes over time. Furthermore, we explored the use of problem-specific fine-tuning of the model by setting some crossvariable correlations to exactly zero (Simplified ConCAR model). This provide us a way to establish certain correlations among different variables as well as simplifying the calculation. In the end, the rate estimates are smoothed rates which can provide us good overviews about spatio-temporal patterns by plotting them on maps.

As mentioned in previous sections, there are many possibilities to extend and improve the MarCAR/ConCAR framework. For example, the precision matrix in a CAR prior (2.3) can be changed to the convolution prior (2.5), the linear time assumption can be replaced with $\mathrm{AR}(1)$ or $\mathrm{AR}(2)$ process and the error term $\boldsymbol{\epsilon}$ can have a structured covariance matrix to account for spatial temporal interaction. A drawback for the MarCAR or ConCAR model is that it requires longer computation 
time compared to recent multivariate disease mapping models. The specific relationship can be studied between the multivariate disease mapping models and the MarCAR/ConCAR models. If exist, one can potentially take advantage of the computation resources from the multivariate disease mapping models and apply them to the misaligned region settings, while incorporating outside information like area, population, etc. 


\section{Chapter 3}

\section{Using BRFSS data to estimate County-level Colorectal Cancer Screening Prevalence in Missouri}

The colorectal cancer screening (CRCS) plays an important role in the early detection of CRC, which can provide patients higher chance of survival. When screening identifies a colorectal tumor in its early stages, the cost of treatment is often much less expensive than if the tumor is detected later in the course of the disease. In this chapter, we applied small area estimation (SAE) techniques to produce county-level CRCS prevalence in Missour based on the state-level BRFSS data. 


\subsection{Introduction}

\subsubsection{Existing surveys covered CRCS in Missouri}

There are two kinds of regularly conducted surveys in Missouri which contain information about CRCS prevalence. One is the Behavioral Risk Factor Surveillance System (BRFSS) and the other one is Missouri County-Level Study.

The Behavioral Risk Factor Surveillance System (BRFSS) is the nation's premier system of health-related telephone surveys that collect state data about U.S. residents regarding their health-related risk behaviors, chronic health conditions, and use of preventive services. Since its creation by the U.S. Centers for Disease Control and Prevention (CDC) in 1984, the BRFSS has been conducted annually in all 50 states as well as the District of Columbia and three U.S. territories. The Health and Behavioral Risk Research Center at the University of Missouri-Columbia collects BRFSS data for Missouri state. To adjust the sampling bias and make sure the data collected are representative of the population for the state, raking (iterative proportional fitting) was used to accomplish this goal. The sample weight for a particular sample generated by raking can be interpreted as the inverse probability of a "likely" or "unlikely" the person been selected. For every two years, questions related to CRCS prevalence are asked in Missouri. For interviewers aged 50 and older, they were asked if they never had a sigmoidoscopy or colonoscopy, which are two major types of CRCS. Based on responses to this question, CRCS prevalence for Missouri can be obtained. We denote the Missouri BRFSS data as MO-BRFSS data. In 2012, there were 5,310 adults interviewed by randomly selected household landline tele-

phone numbers. Additionally, 1,403 randomly selected adult cellphone-mostly users 
participated in the interview. A CRCS prevalence of $66.5 \%$ was reported for Missouri.

The Missouri County-level Study, which we denote as MO-CLS, followed standard CDC BRFSS methods and techniques. The questionnaire used for the interviews contains questions from the BRFSS and CDC Adult Tobacco Survey (ATS). Different from MO-BRFSS which only aims state-level estimates, MO-CLS aims at producing accurate county-level estimates by collecting much more data for each county. For example, the 2011 CLS goal was to complete 47,200 landline interviews with Missouri adults ages 18 and older. The goals were as follows:

- 800 interviews in Jackson County and St. Louis County with 400 interviews among African Americans and other races and 400 interviews among whites.

- 800 interviews in St Louis City with 400 interviews among African Americans and 400 interviews among whites and other races.

- 400 completed interviews in the rest of 112 counties.

Additionally, a goal was established to obtain 4,720 interviews with adult cellphoneonly users. Data from cell phone interviews were combined with landline data for analysis at the state and regional levels. Between January and December 2011, interviews were completed with 47,261 landline and 4,828 cell phone only users, totaling 52,089 completed interviews. The same raking method was used for MO-CLS to produce weighting variables. Note that the weighting variable in MO-BRFSS is to make survey sample represent the whole Missouri State, while the weighting variable in MO-CLS makes the survey sample represent each county in Missouri, which produce feasible ways to conduct county-level estimates. For interviewers aged 50 and older, they were asked if they never had a sigmoidoscopy or colonoscopy, which 
is the same as the question asked in MO-BRFSS. At the state level, 2011 MO-CLS reported CRCS prevalence as $66.2 \%$, which is close to 2012 MO-BRFSS result. The MO-CLS is ideal for producing county-level prevalence estimates, but it is conducted less frequently as MO-BRFSS. Currently, MO-CLS were only conducted for years 2003, 2007, 2011 and 2016.

\subsubsection{Aim of the study}

It is useful to get county-level CRCS prevalence estimates and MO-CLS can directly serve the purpose. However, the discontinuity in conducting years limits us from monitoring changes over time. MO-BRFSS can give us the state-level estimates every two years, but not for the county-level estimates. There are many counties in Missouri have zero or rather small sample sizes in MO-BRFSS data, which prevent us from getting county-level estimates directly. In this paper, we want to examine the possibilities of using MO-BRFSS data to produce county-level estimates. The process of estimating prevalences for counties with no samples falls into the category of small area estimation (SAE) problems. Rao (2015) is a classical text contains various methods for SAE problems. SAE techniques have been widely used in survey context when one tries to estimate the quantity of interest for an area with no sample available. When applying SAE methods, the survey sampling design should be considered to reduce biases in the estimates.

Once county-level estimates were obtained from MO-BRFSS data, we will use MOCLS to evaluate the results. The county information is no longer publicly available for MO-BRFSS data after 2012. In addition, the 2016 MO-CLS data is not available when the study was conducted. Due to these limitations, we decided to use 2011 MO- 
CLS data and 2012 MO-BRFSS data as two sources to produce county-level CRCS prevalence estimates. The state-level estimate in Section 3.1.1 shows little difference between 2011 MO-CLS and 2012 MO-BRFSS. Thus we assume the prevalence stay unchanged from 2011 to 2012. Due to much large sample sizes in 2011 MO-CLS, its estimates are treated as "true" values. Estimates from MO-BRFSS with SAE methods will be compared to those "true" values and results will be evaluated.

\subsubsection{Review of SAE methods for surveys}

We briefly review some SAE methods used for survey analysis which have already been proposed in the literature. As in a typical survey analysis, population totals or

population proportions (prevalences) are often quantities of interest. Our purpose is to use survey samples to estimate population truth. There are two types of approaches used in practice. The first one is the design-based approach and the other one is the model-based approach.

The design-based approach is carried out based on distributions of the individuals that could appear in the survey. Weighting variables described in Section 3.1.1 can be interpreted as the inverse of the inclusion probability for each individual. In MO-BRFSS or MO-CLS, the weights obtained from raking are normalized so that the sum of the weights equals the true population size. In other words, the weights for one individual means how many people he (she) is representing for in the whole population. Design-consistent estimators can be generated by the weighted average/sum of sample quantities based on weighting variables. The Horvitz-Thompson (HT) estimator (Horvitz and Thompson, 1952) is commonly used in practice. Let $Y_{i k}$ be a binary health outcome for individual $k$ in county $i\left(i=1, \ldots, I\right.$ and $\left.k=1, \ldots, N_{i}\right)$, 
where $N_{i}$ is the population size for county $i$ and usually known. In a survey, a sample of size $n_{i}$ is drawn from each county $i$ with sample values $y_{i k}$. The true prevalence for each county $i$, denoted as $P_{i}$, can be calculated as

$$
P_{i}=\frac{1}{N_{i}} \sum_{k=1}^{N_{i}} Y_{i k} .
$$

Suppose the calculated inclusion probability for individual $k$ in county $i$ is $\pi_{i k}$ and the weight is $w_{i k}=1 / \pi_{i k}$. For normalized weights, $\sum_{k=1}^{N_{i}} w_{i k}=N_{i}$. Then the HT estimator of $P_{i}$ is given by

$$
\hat{p}_{i}^{H T}=\frac{1}{N_{i}} \sum_{k=1}^{N_{i}} \frac{y_{i k}}{\pi_{i k}},
$$

with variance

$$
\widehat{\operatorname{Var}}\left(\hat{p}_{i}^{H T}\right)=\frac{1}{N_{i}^{2}}\left[\sum_{k=1}^{N_{k}}\left(\frac{1-\pi_{i k}}{\pi_{i k}^{2}}\right) y_{i k}^{2}+\sum_{k=1}^{N_{k}} \sum_{k \neq k^{\prime}}\left(\frac{\pi_{i k, i k^{\prime}}-\pi_{i k} \pi_{i k^{\prime}}}{\pi_{i k} \pi_{i k^{\prime}}}\right) \frac{y_{i k} y_{i k^{\prime}}}{\pi_{i k, i k^{\prime}}}\right],
$$

where $\pi_{i k, i k^{\prime}}$ is the sampling probability for the pair of individuals $i k$ and $i k^{\prime}$. For a description of constructing $\pi_{i k, i k^{\prime}}$, see Lumley (2011). The HT estimator is a designunbiased estimator of $P_{i}$ and often called the direct estimator since it only uses the responses from the area of interest. One limitation for HT method is that the estimator can not be calculated for area with no samples. Other design-based methods use a model for construction of the estimators. Pfeffermann et al. (2013) contains an overview of recent developed design-based methods which can produce estimates for non-sampled areas.

The model-based approach assumes a hypothetical infinite population from which the responses are drawn. The model-based approach is appealing since standard 
statistical modeling machinery can be applied. However, it is difficult to implement since the sampling mechanism needs to be modeled. One needs to include all variables used in the sampling process, which are often not available. Even if available, the complex survey itself is hard to model. Gelman (2007) describes the issues. Despite of the issues, developing model-based approaches has been an active research area. Mercer et al. (2014) compares several existing methods. The simplest model is the binomial regression model which assumes

$$
y_{i} \mid P_{i} \sim \operatorname{Binomial}\left(n_{i}, P_{i}\right) \text { and } \operatorname{logit}\left(P_{i}\right)=\beta_{0}+u_{i}+\nu_{i}
$$

where $y_{i}=\sum_{k=1}^{n_{k}} y_{i k}, \beta_{0}$ is the overall intercept, $u_{i}$ is the spatial effect for county $i$ and $\nu_{i}$ is random error, which is similar to a BYM model (Besag et al., 1991). However, such model completely ignores the survey design. Many methods has been proposed for SAE methods which account for survey design by either include the variance estimate $\widehat{\operatorname{Var}}\left(\hat{p}_{i}^{H T}\right)$ as part of the model, for example the logit-normal model (Mercer et al., 2014), the arcsine model (Raghunathan et al., 2007), or directly modeling the sample weighting variable, for example using penalized splines (Vandendijck et al., 2016), or combining pseudo-likelihood with the effective sample size calculated by weighting variable (Chen et al., 2014), or just regressing the response variable on a Gaussian process function of the weighting variable (Si et al., 2015). There are still many other approaches that we have not mentioned.

Unfortunately, the weighting variable is not available in MO-BRFSS data in terms of producing county-level estimates. Thus the model-based approaches mentioned above with weights being part of the model can not be applied. Similar to our situation, Cadwell et al. (2010) used Bayesian multilevel models to estimate diabetes 
prevalences for 3,141 counties in the US using BRFSS data. Estimating MO countylevel CRCS prevalences is essentially the same problem. Survey respondents were classified into $J$ categories based on age, race and sex. Because of low prevalences of diabetes (5.5\% national wide), they assume the number of sample people who have diabetes for county $i$ and category $j$, namely $y_{i j}$ follows a Poisson distribution

$$
y_{i j} \mid P_{i j} \sim \operatorname{Poisson}\left(n_{i j} P_{i j}\right) ; \quad i=1, \ldots, I \quad \text { and } \quad j=1, \ldots, J
$$

where $I$ is the number of counties, $n_{i j}$ is the sample size and $P_{i j}$ is the prevalence for category $j$ of county $i$. They further model $P_{i j}$ through

$$
\log \left(P_{i j}\right)=\beta_{i j}
$$

where $\boldsymbol{\beta}_{i}=\left(\beta_{i 1}, \ldots, \beta_{i J}\right)^{\prime}$ follows a multivariate normal distribution with mean $\mu_{s[i]}$

and covariance matrix $\boldsymbol{\Sigma}$. Here $s[i]$ denote the state where county $i$ belongs to. They assumed independent normal priors on $\mu_{s[i]} \mathrm{s}$ and Inverse-Wishart prior on $\boldsymbol{\Sigma}$. From the posterior samples, the posterior predictive distributions of county-level prevalence $P_{i}$ was obtained with the use of true population sizes from Census data. The mean of the posterior predictive distribution was used as the estimate of $P_{i}$.

\subsubsection{Outline of the chapter}

We used Cadwell et al. (2010) as our main reference with modifications to address our problem. Firstly, we assumed binomial distribution for $y_{i j}$ since the CRCS prevalence is around $60 \%$ in our case, where Poisson approximation may not work well. Secondly, we avoided modeling the dependence between different categories of people 
and assigning an Inverse-Wishart prior on the covariance matrix. Compared to their data, we only have 115 counties with relatively much smaller sample size. Thus the covariances between different categories will be hard to estimate. For simplicity, we assumed independence among categories. Lastly, we added county-level covariates: the percentage of people below high school education level, the percentage of people below 9th-grade education level, the percentage of people above bachelor education level and the median house income. The inclusion of these variables improved our estimates.

In Section 3.2, we provide details of the 2012 MO-BRFSS data and the process of getting county-level estimates from a Bayesian hierarchical model. The results are evaluated in Section 3.3 and followed by discussions in Section 3.4.

\subsection{Methods}

\subsubsection{Data}

Missouri is comprised of 114 counties and the City of St. Louis. For the 2012 MOBRFSS data, we only selected people over 50 years old as 50 is the recommended age to start CRCS, which left us with 4,605 samples. After removing unknown counties and unknown responses about CRCS, we had a total of 3,807 samples for analysis. In MO-BRFSS study, the sample size from each county was too small to make conclusive estimates by county. Therefore, those areas were clustered into seven MO-BRFSS regions shown in Figure 3.1. Table 3.1 contains the sample size for each region. These seven regions are the smallest area units where survey results are reported. 


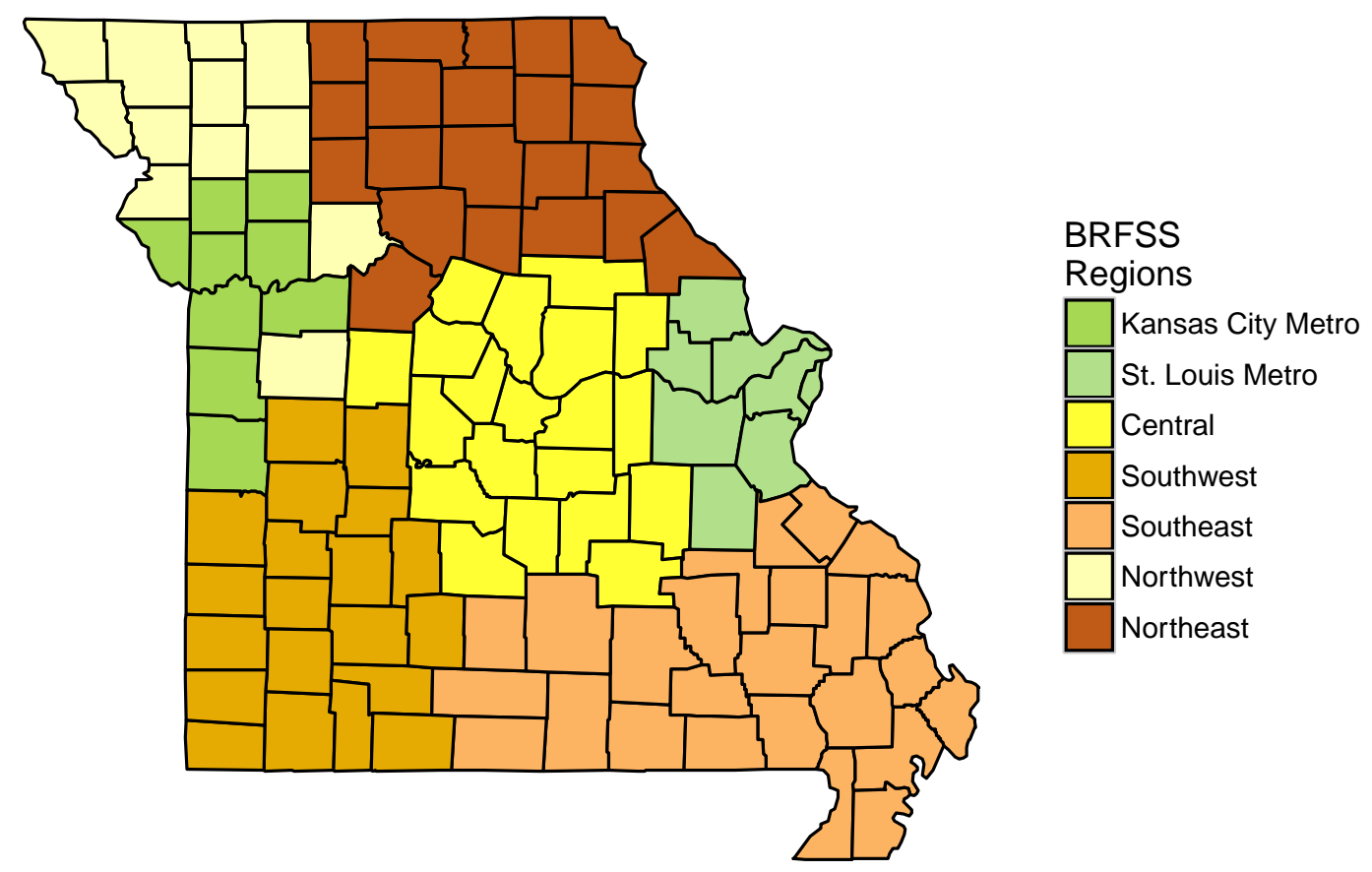

Figure 3.1: 2012 MO-BRFSS Regions.

Figure 3.2 shows the sample sizes for each county. Although 3,807 observations seem quite a lot at the state level, it is not enough to get estimates for every county since most samples were from a highly populated urban areas, in which case we have highly unbalanced distribution of survey samples across all counties. For example, there were 37 counties in MO have zero sample (white color) and only 15 counties had more than 50 samples, where 50 is the minimum sample size that CDC will report the prevalence for a Chronic Disease indicator. Therefore, getting accurate county-level CRCS prevalence estimates is quite challenging.

For 2011 MO-CLS data, more samples were obtained for each county. After cleaning the data, we have 35,590 samples for people over 50 years old with valid 


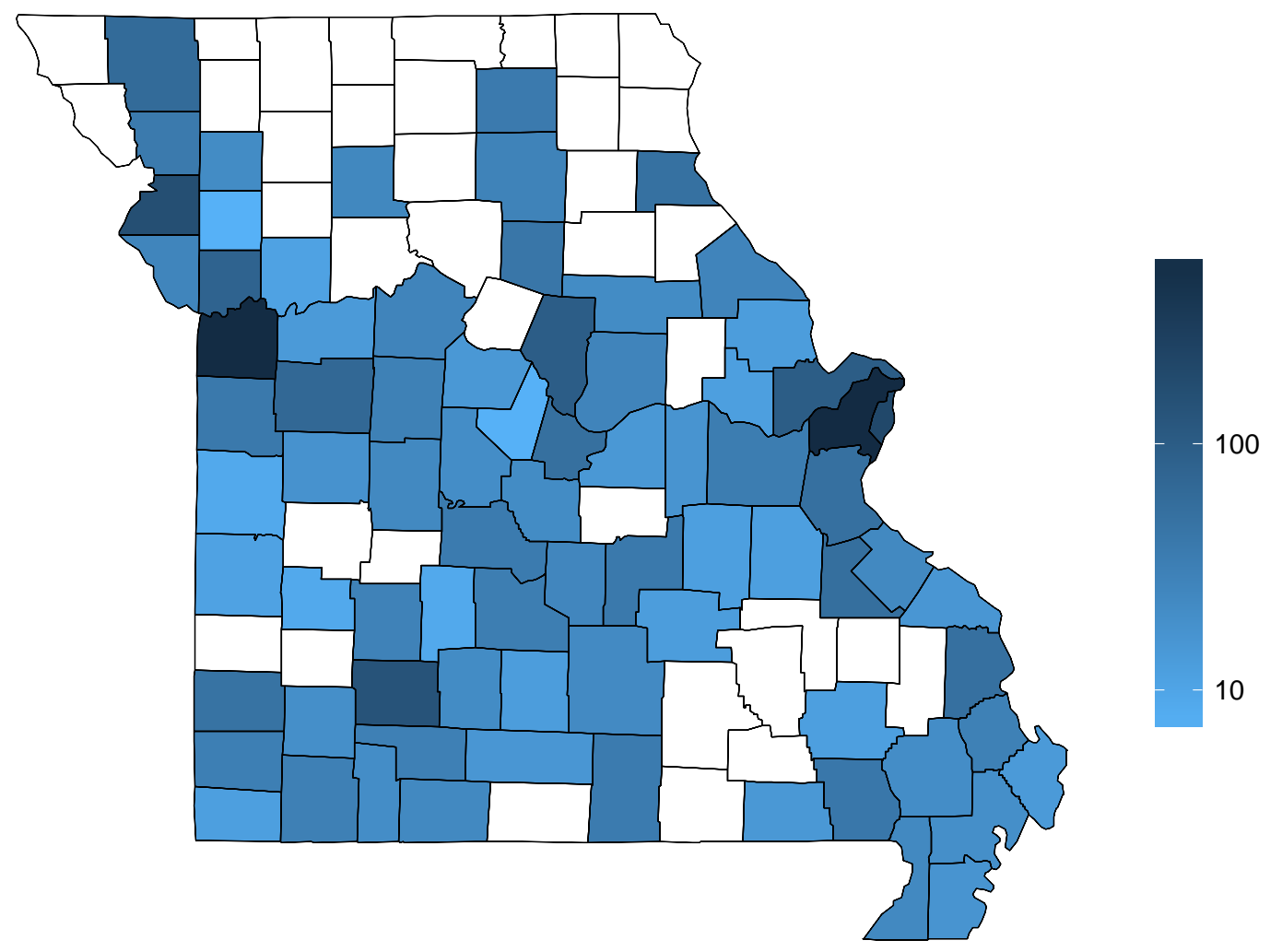

Figure 3.2: 2012 MO-BRFSS Sample Sizes Counties in Missouri 
Table 3.1: 2012 MO-BRFSS Regions in MO with Sample Sizes

\begin{tabular}{lr}
\hline Region & Sample size \\
\hline Kansas City Metro & 767 \\
St. Louis Metro & 1030 \\
Central & 493 \\
Southwest & 485 \\
Southeast & 433 \\
Northwest & 355 \\
Northeast & 244 \\
\hline
\end{tabular}

county and demographic information. The sample sizes for all counties ranged from 209 to 706, with a median 300. Weighting variables were also created for countylevel estimates in this survey. The availability of the weighting variable allows us to get direct county-level estimates using the HT estimator. With large sample size in MO-CLS, we may assume the direct estimates are accurate.

The U.S. Census Bureau publishes population estimates by demographic characteristics for all counties. We used 2012 Census county projections to obtain the estimates for the population size for different demographic categories in each county in Missouri, which were used for cross-classify 2012 MO-BRFSS data later on. Here we ignore the uncertainty of the population sizes. These population size information will be used to adjust our estimates to match the population distribution for each county.

The county attribute variables can be obtained using SEER*Stat software. It provides a convenient, intuitive mechanism for the analysis of Surveillance, Epidemiology, and End Results Program (SEER) and other cancer-related databases. The SEER*Stat calculated the county attribute variables for 2010-2014 based on the Census American Community Survey (ACS) 5-year files. We used the five years 2010 to 2014 as the year 2012 is the middle year of the span, which we would expect less bias. 
Note that year 2012 is the one we want to study.

\subsubsection{Prevalence estimator}

Respondents in MO-BRFSS were classified into different categories based on their county at diagnosis, age $(50-64,65-74,75+)$, gender (male, female) and race (white, non-white). For each county $i, i=1, \ldots, I$ where $I=115$ in Missouri, the three age groups, two genders and two races yield twelve different categories. Let $J=12$ be the total number of categories and we use the following notations for each category $j, j=1, \ldots, J$, in county $i$ :

- $n_{i j}$ : the MO-BRFSS sample size, which is the number of respondents;

- $y_{i j}$ : the number of respondents who have had CRCS out of all $n_{i j}$ respondents;

- $N_{i j}$ : the true population size based on 2010 Census data;

- $Y_{i j}$ : the true population total who have had CRCS out of $N_{i j}$ people;

- $P_{i j}$ : the true proportion of people who have had CRCS.

In the variables above, $n_{i j}$ and $y_{i j}$ are known through our MO-BRFSS data. If $n_{i j}=0$, we set $y_{i j}=0 . N_{i j}$ is also known from 2010 Census data. The other two quantities $Y_{i j}$ and $P_{i j}$ are unobserved. For county $i$, the unobserved prevalence is:

$$
P_{i}=\frac{\sum_{j=1}^{J} Y_{i j}}{\sum_{j=1}^{J} N_{i j}},
$$

which is the quantity we are interested in. 
We define $Z_{i j}=Y_{i j}-y_{i j}$ to be the total number of people in 2012 who have had CRCS in county $i$ category $j$ but not included in the survey. Then (3.7) is equivalent to

$$
P_{i}=\frac{\sum_{j=1}^{J}\left(Z_{i j}+y_{i j}\right)}{\sum_{j=1}^{J} N_{i j}} .
$$

\subsubsection{Bayesian binomial regression}

We used a Bayesian binomial regression framework to estimate $P_{i j}$. To be specific, we assume $y_{i j}$ follows a Binomial distribution with number of trials $n_{i j}$ and success probability $P_{i j}$ :

$$
y_{i j} \sim \operatorname{Binomial}\left(n_{i j}, P_{i j}\right) .
$$

A logit transformation $v_{i j}=\log \left(P_{i j} /\left(1-P_{i j}\right)\right)$ was used in the second level regression. With all available covariates, $v_{i j}$ can be modeled as:

$$
v_{i j}=\mu+\alpha_{r(i)}+\beta_{j}+\phi_{1} x_{1 i}+\phi_{2} x_{2 i}+\phi_{3} x_{3 i}+\phi_{4} x_{4 i}+u_{i}+\epsilon_{i j},
$$

where

- $\mu$ is the overall intercept;

- $\alpha_{r(i)}$ is the regional effect of region $r(i) \in\{1,2, \ldots, 7\}$ (recall that we have seven BRFSS regions for 2012 MO-BRFSS);

- $\beta_{j}$ is effect for the $j$ th demographic category;

- $x_{1 i}$ is the percentage of people below high school education level, with coefficient $\phi_{1}$ 
- $x_{2 i}$ is the percentage of people below 9th-grade education level, with coefficient $\phi_{2}$

- $x_{3 i}$ is the percentage of people above bachelor education level, with coefficient $\phi_{3}$

- $x_{4 i}$ is the median house income, with coefficient $\phi_{4}$;

- $u_{i}$ is a random spatial effect which accounts for extra county level variability not included in our model if exist;

- $\epsilon_{i j k l}$ is the overdispersion term accounting for extra variability not included in our model.

For $\alpha_{r(i)} \mathrm{s}$ and $\beta_{j} \mathrm{~s}$, sum-to-zero constraints are added for identifiability issues.

It is helpful to write our model in vector notation. We define $\boldsymbol{y}=\left(\boldsymbol{y}_{1}^{\prime}, \ldots, \boldsymbol{y}_{I}^{\prime}\right)^{\prime}$ where $\boldsymbol{y}_{i}=\left(y_{i 1}, \ldots, y_{i J}\right)^{\prime}$. The vectors $\boldsymbol{n}, \boldsymbol{N}, \boldsymbol{P}$ and $\boldsymbol{v}$ are defined in the same way as $\boldsymbol{y}$. The likelihood function (3.9) can then be rewritten as:

$$
\boldsymbol{y} \sim \operatorname{Binomial}(\boldsymbol{n}, \boldsymbol{P}) .
$$

For the regression (3.10), we define a $I J \times 7$ design matrix $\boldsymbol{X}_{\alpha}$ with each row being an indicator where only the $r(i)$ th element is one and all remaining elements are zeros. We collect $\boldsymbol{\alpha}=\left(\alpha_{1}, \ldots, \alpha_{7}\right)^{\prime}$. Then $\boldsymbol{X}_{\alpha} \boldsymbol{\alpha}$ gives a vector to indicate the region each element in $\boldsymbol{v}$ belongs to. By the same methods, we create a $I J \times J$ design matrix $\boldsymbol{X}_{\beta}=\boldsymbol{I}_{J} \otimes \mathbf{1}_{I}$ with vector $\boldsymbol{\beta}=\left(\beta_{1}, \ldots, \beta_{J}\right)^{\prime}$ where $\boldsymbol{I}_{J}$ is an identity matrix of size $J, \mathbf{1}_{I}$ is a vector with all ones of size $I$ and $\otimes$ is the Kronecker product. For the county attributes, let $\boldsymbol{\phi}=\left(\phi_{1}, \phi_{2}, \phi_{3}, \phi_{4}\right)^{\prime}$ and $\boldsymbol{x}_{i}=\left(x_{1 i}, x_{2 i}, x_{3 i}, x_{4 i}\right)^{\prime}$, then 
$\phi_{1} x_{1 i}+\phi_{2} x_{2 i}+\phi_{3} x_{3 i}+\phi_{4} x_{4 i}=\boldsymbol{x}_{i}^{\prime} \boldsymbol{\phi}$. The design matrix for $\boldsymbol{\phi}$ can be written as $\boldsymbol{X}_{c}=\mathbf{1}_{J} \otimes\left(\boldsymbol{x}_{1}, \ldots, \boldsymbol{x}_{I}\right)^{\prime}$. For the random spatial effect $u_{i}$, let $\boldsymbol{u}=\left(u_{1}, \ldots, u_{I}\right)^{\prime}$. The design matrix for $\boldsymbol{u}$ is $\boldsymbol{X}_{u}=\mathbf{1}_{J} \otimes \boldsymbol{I}_{I}$. Under all these definitions, model (3.10) can be rewritten as

$$
\boldsymbol{v}=\mu \mathbf{1}_{I J}+\boldsymbol{X}_{\alpha} \boldsymbol{\alpha}+\boldsymbol{X}_{\beta} \boldsymbol{\beta}+\boldsymbol{X}_{c} \boldsymbol{\phi}+\boldsymbol{X}_{u} \boldsymbol{u}+\boldsymbol{\epsilon}
$$

where $\boldsymbol{\epsilon}$ a size $I J$ vector collects all $\epsilon_{i j}$ in the same way as $\boldsymbol{v}$.

\subsubsection{Prior distributions on the regression parameters}

Flat priors are used for parameters $\mu, \boldsymbol{\alpha}, \boldsymbol{\beta}$, and $\boldsymbol{\phi}$. We assume independence among all these parameters. Normal distributions with large variances are used as their prior distributions:

$$
\mu \sim N(0, \gamma), \quad \boldsymbol{\alpha} \sim N_{7}\left(\mathbf{0}_{7}, \gamma \boldsymbol{I}_{7}\right), \quad \boldsymbol{\beta} \sim N_{J}\left(\mathbf{0}_{J}, \gamma \boldsymbol{I}_{J}\right), \quad \boldsymbol{\phi} \sim N_{4}\left(\mathbf{0}_{4}, \gamma \boldsymbol{I}_{4}\right)
$$

where $N_{m}(\cdot, \cdot)$ indicate a multivariate normal distribution of dimension $m, \mathbf{0}_{m}$ is a length $m$ vector of zeros and $\boldsymbol{I}_{m}$ is an $m$ dimensional identity matrix. Here we use a large variance $\gamma$ for the purpose of non-informative priors.

For the over-dispersion parameter $\boldsymbol{\epsilon}$, we assume

$$
\boldsymbol{\epsilon} \sim N_{I J}\left(\mathbf{0}_{I J}, \delta_{0} \boldsymbol{I}_{I J}\right)
$$

where $\delta_{0}$ is the variance parameter for $\boldsymbol{\epsilon}$.

We used a CAR model for the spatial effect $\boldsymbol{u}=\left(u_{1}, \ldots, u_{I}\right)^{\prime}$ in our analysis. We have a brief review here. For each $u_{i}$ defined at county $i$, the CAR model assumes it 
has at least one neighboring county. We use $i^{\prime} \sim i$ to denote that county $i^{\prime}$ is adjacent to $i$. Based on the locations for all the counties, let $\boldsymbol{C}=\left(c_{i^{\prime} i}\right)_{I \times I}$ be an adjacency matrix describes the neighboring structure of the counties in MO. The element $c_{i^{\prime} i}=1$ if county $i^{\prime}$ adjacent to county $i$ and $c_{i^{\prime} i}=0$ otherwise. By convention, a county will not be the neighbor of itself so we define $c_{i i}=0$. Then the CAR model specifies the conditional densities of $u_{i}$ given all the other variables to be:

$$
\left(u_{i} \mid u_{i^{\prime}}, i^{\prime} \neq i\right) \sim N\left(\rho \sum_{i^{\prime} \sim i} u_{i^{\prime}}, \delta\right)
$$

where $\delta>0$ specifies the conditional variance, $\sum_{i^{\prime} \sim i} u_{i^{\prime}}$ is the sum of neighboring variables around county $i$, and $\rho$ specifies the strength of the relationship between county $i$ and its neighbors. It has been shown that (3.15) is equivalent to the multivariate normal distribution (Sun et al., 1999)

$$
[\boldsymbol{u} \mid \delta, \rho] \sim N_{I}\left(\mathbf{0}, \delta(\boldsymbol{I}-\rho \boldsymbol{C})^{-1}\right) .
$$

The matrix $\delta(\boldsymbol{I}-\rho \boldsymbol{C})^{-1}$ need to be positive definite, which requires $\rho$ in the range:

$$
\rho \in\left(\frac{1}{\min \nu_{i}}, \frac{1}{\max \nu_{i}}\right),
$$

where the $\nu_{i}$ s are eigenvalues of the adjacency matrix $\boldsymbol{C}$. For counties in Missouri, the range for $\rho$ is $\left(\rho_{\min }, \rho_{\max }\right)$, where $\rho_{\min }=-0.347$ and $\rho_{\max }=0.173$. 


\subsubsection{Selection of hyperpriors}

Parameters in the priors distribution in Section 3.2.4 need to be specified for a full Bayesian analysis. The variance parameter $\delta_{0}$ in (3.14) was given an Inverse$\operatorname{Gamma}\left(a_{0}, b_{0}\right)$ prior distribution with density proportional to

$$
\left[\delta_{0} \mid a_{0}, b_{0}\right] \propto \frac{1}{\tau_{1}^{a_{0}-1}} \exp \left(-\frac{b_{0}}{\delta_{0}}\right), \quad \text { for } \delta_{0}>0
$$

Here we use $\left[\delta_{0} \mid a_{0}, b_{0}\right]$ to denote the probability density function of $\delta_{0}$ conditional on $a_{0}$ and $b_{0}$.

The parameter $\rho$ controls the strength of spatial association and is restricted in (3.17). Thus we used an uniform prior distribution

$$
\rho \sim \operatorname{Unif}\left(\rho_{\min }, \rho_{\max }\right) .
$$

To complete our hierarchical model, we still need a prior on the variance parameter $\delta$ in (3.16). Instead of assigning a prior distribution on $\delta$ independently from $\delta_{0}$, we connect $\delta_{0}$ with $\delta$ by the noise-to-signal ratio $\eta=\delta_{0} / \delta$ in the same way as in (2.19) and (2.20). To be specific, a scaled Pareto prior on $\eta$ was used,

$$
[\eta \mid a]=\frac{a}{(a+\eta)^{2}}, \quad \eta>0 .
$$

The benefit of using noise-to-signal ratio has been discussed in Chapter 5.3.5. In this application, we choose $a=1$ so that

$$
[\phi \mid a=1] \sim \operatorname{Unif}(0,1)
$$


which treats the spatial variation and unstructured error variation equally likely.

\subsubsection{Estimates of CRCS prevalence}

Recall in (3.8) $Z_{i j}$ is needed for county-level CRCS prevalence estimates. From our hierarchical model, the posterior predictive distribution of $Z_{i j}$ is

$$
Z_{i j} \mid \boldsymbol{y}, \boldsymbol{n}, \boldsymbol{N} \sim \operatorname{Binomial}\left(N_{i j}-n_{i j}, P_{i j}\right)
$$

with expected value $E\left[Z_{i j} \mid \boldsymbol{y}, \boldsymbol{n}, \boldsymbol{N}\right]=\left(N_{i j}-n_{i j}\right) P_{i j}$. Then the prevalence can be estimated as

$$
\begin{aligned}
\hat{P}_{i}=E\left(P_{i} \mid \boldsymbol{y}, \boldsymbol{n}, \boldsymbol{N}\right) & =E\left(\frac{\sum_{j=1}^{J}\left(Z_{i j}+Y_{i j}\right)}{\sum_{j=1}^{J} N_{i j}} \mid \boldsymbol{y}, \boldsymbol{n}, \boldsymbol{N}\right) \\
& =\frac{\sum_{j=1}^{J}\left[E\left[Z_{i j} \mid \boldsymbol{y}, \boldsymbol{n}, \boldsymbol{N}\right]+Y_{i j}\right]}{\sum_{j=1}^{J} N_{i j}} .
\end{aligned}
$$

Posterior distributions of $P_{i j}$ can be obtained from our Bayesian hierarchical models, thus posterior predictive distribution of $\hat{P}_{i}$ can also be obtained.

\subsection{Results}

We fit the model described in Section 3.2 with 2012 MO-BRFSS data. We treat the regression model (3.12) for the linear predictor $\boldsymbol{v}$ as our full model, which contains all covariates available in our analysis: the demographic information, county attributes and random spatial effects. However, not all covariates may be needed to produce reasonable estimates. We want to know how good the estimates are with the absence 
of some covariates. Three additional models with fewer covariates were fitted. Table 3.2 contains the forms of all different models we checked. Model 1 is our full model contains all covariates. In Model 2 we remove the random county effect. This is considered because with the presence of county attributes and the sparsity of our data, the random county effect can be hard to estimate. Model 3 only contains the demographic covariates and the random county effect, which mimics the situation where county attributes are not available. In the end, Model 4 is the simplest model including only the demographic covariates. Note that the regional effects were retained for all models.

Table 3.2: Models with different covariates

\begin{tabular}{llr}
\hline & Covariates & $\boldsymbol{v}$ \\
\hline Model 1 & demographic + county attributes + spatial effect & $\mu \mathbf{1}_{I J}+\boldsymbol{X}_{\alpha} \boldsymbol{\alpha}+\boldsymbol{X}_{\beta} \boldsymbol{\beta}+\boldsymbol{X}_{c} \boldsymbol{\phi}+\boldsymbol{X}_{3} \boldsymbol{u}+\boldsymbol{\epsilon}$ \\
Model 2 & demographic + county attributes & $\mu \mathbf{1}_{I J}+\boldsymbol{X}_{\alpha} \boldsymbol{\alpha}+\boldsymbol{X}_{\beta} \boldsymbol{\beta}+\boldsymbol{X}_{c} \boldsymbol{\phi}+\boldsymbol{\epsilon}$ \\
Model 3 & demographic + spatial effect & $\mu \mathbf{1}_{I J}+\boldsymbol{X}_{\alpha} \boldsymbol{\alpha}+\boldsymbol{X}_{\beta} \boldsymbol{\beta}+\boldsymbol{X}_{3} \boldsymbol{u}+\boldsymbol{\epsilon}$ \\
Model 4 & demographic & $\mu \mathbf{1}_{I J}+\boldsymbol{X}_{\alpha} \boldsymbol{\alpha}+\boldsymbol{X}_{\beta} \boldsymbol{\beta}+\boldsymbol{\epsilon}$ \\
\hline
\end{tabular}

\subsubsection{Computation}

An Markov Chain Monte Carlo (MCMC) algorithm was used to generate the posterior distributions of parameters in our model. A Gibbs sampling algorithm was implemented in $\mathrm{R}$ and $\mathrm{C}++$ with the full conditional densities derived in Appendix B.2. The full conditional distribution for $\boldsymbol{\psi}=\left(\mu, \boldsymbol{\alpha}^{\prime}, \boldsymbol{\beta}^{\prime}, \boldsymbol{\phi}^{\prime}\right)^{\prime}$ is multivariate normal distributions. The full conditional distribution for $\delta_{0}$ is inverse-gamma distributions. These random variables can be sampled directly with available softwares. For all the other parameters, Adaptive Rejection Metropolis Sampling (ARMS) introduced in Gilks et al. (1995) was used to get the posterior samples via a $\mathrm{C}++$. In our 
application, the following hyperparameter values were used:

$$
a_{0}=b_{0}=a=1, \quad \gamma=10^{4} .
$$

We used 20,000 posterior samples for all models in Table 3.2 after discarding the first 10,000 ones. Convergences were monitored via trace plots and posterior density plots.

\subsubsection{Model evaluation}

In our application, 20,000 posterior samples of $\hat{P}_{i}$ were obtained for each county $i$ based on the posterior predictive distribution in (3.23). The mean $\hat{p}_{i}$ of the posterior predictive distribution was used as the estimate of $P_{i}$. We define $\hat{\boldsymbol{p}}=\left(\hat{p}_{1}, \ldots, \hat{p}_{I}\right)^{\prime}$ to be the estimates for all counties from MO-BRFSS. The direct HT estimates of $P_{i}$ s from MO-CLS were also obtained as our "true" values for comparison and we denote them as $\boldsymbol{p}^{C L S}=\left(p_{1}^{C L S}, \ldots, p_{I}^{C L S}\right)^{\prime}$. Figure 3.3 contains the scatter plots of estimates from MO-BRFSS against CLS for Models 1, 2, 3 and 4. The closer are the points from the diagonal line, the better our estimates are.

To evaluate the performance of different models in Table 3.2, we measure the closeness between the estimates from one of the four models and the estimates from CLS. We used the mean absolute difference (MAD), the Pearson correlation and Spearman correlation as three metrics. Table 3.3 contains the results for all model. Smaller values of MAD and larger values of Pearson/Spearman correlation will indicate $\hat{\boldsymbol{p}}$ is closer to $\boldsymbol{p}^{C L S}$. Firstly, we notice that the MAD and Pearson Correlations are consistent with each other. A model with a smaller MAD value will have a Pearson larger correlation value. However, the Spearman correlation shows a different trend 
when comparing Models 1 and 2, or Models 3 and 4. Secondly, by comparing Models 1 and 2 with Models 3 and 4, we notice the presence of county attributes covariates improved our estimates. Thirdly, by comparing Model 1 with Model 2, or Model 3 with Model 4, we notice that the inclusion of the random county effect performs slightly worse in terms of MAD and Pearson correlation. However, the random county effect slightly increases the Spearman correlation. This can be noticed from Figure 3.3 as the points for Models 1 and 3 spread more evenly along the diagonal line than Models 2 and 4. But the improvement is rather small. In situations where a large amount of counties with zero or small sample sizes, the spatial effects are hard to make a difference. However, it does not hurt much to include random spatial effect when prediction is our main interest. Finally, Models 1 and 2 performs the best based on our criteria and the differences between them is negligible. For illustration, we mapped the CRCS prevalences estimates from Model 1 in Figure 3.4, which also contains the spatial plots of the estimates from CLS for compression. The white color represents the statewide CRCS prevalence estimated from CLS, which is around 0.66. In general, estimates from Model 1 differer from CLS by 0.05118 (or 5.118\%) on average. Accurate county prevalence estimates may not be obtained solely from MO-BRFSS data. However, the spatial variation of CRCS prevalence for all counties can be reflected by MO-BRFSS data if we comparing the two spatial plots in Figure 3.4 .

Table 3.3: Model Evaluation

\begin{tabular}{llrr}
\hline & MAD & Pearson & Spearman \\
\hline Model 1 & 0.05118 & 0.582 & 0.606 \\
Model 2 & 0.04949 & 0.591 & 0.603 \\
Model 3 & 0.05894 & 0.487 & 0.514 \\
Model 4 & 0.05887 & 0.491 & 0.507 \\
\hline
\end{tabular}


(a) Model 1

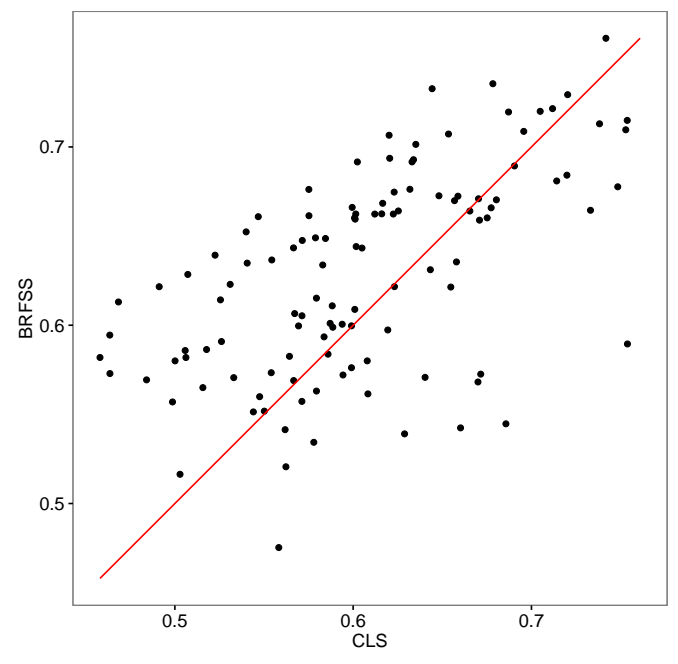

(c) Model 3

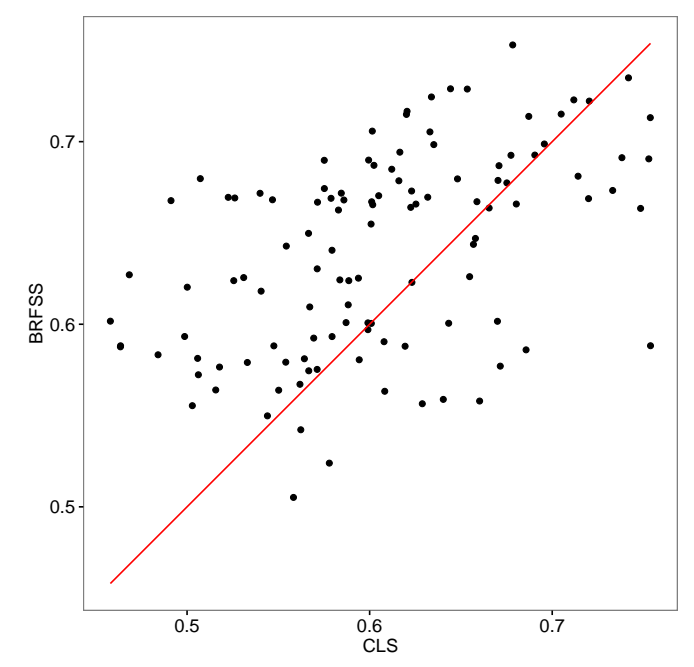

(b) Model 2

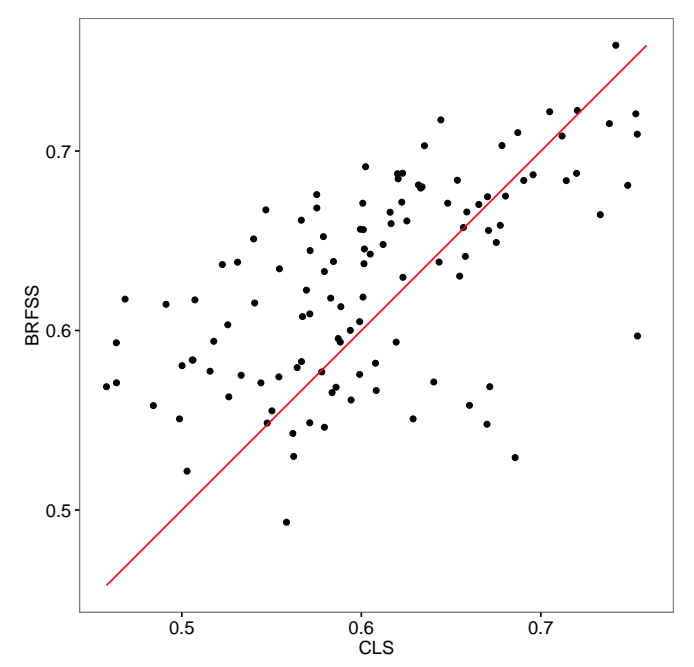

(d) Model 4

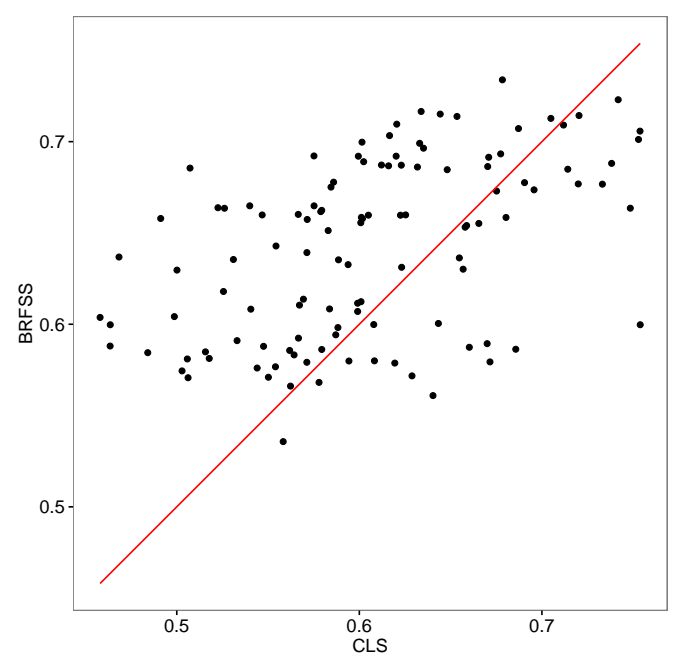

Figure 3.3: Scatter Plots of Estimates from MO-BRFSS Versus CLS for Models 1, 2, 3 and 4 . 
CLS

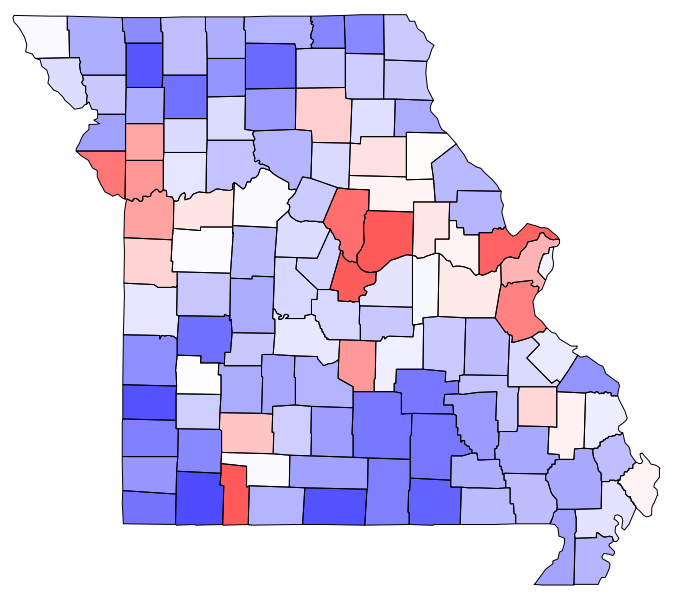

0.46
BRFSS
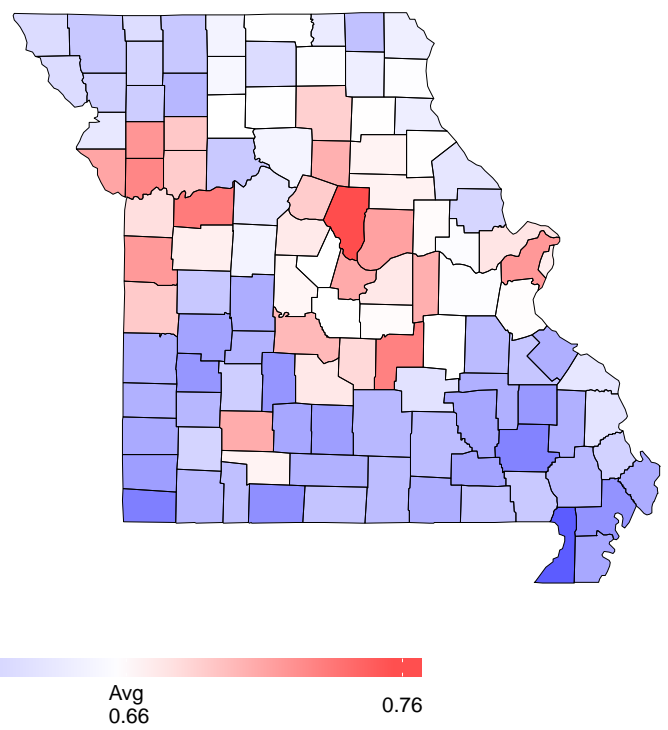

Figure 3.4: Spatial Plots for CRCS Prevalence Estimated from CLS and MO-BRFSS Model 2

\subsection{Discussion}

In this chapter, we explored the possibilities of utilizing MO-BRFSS data for countylevel CRCS prevalence study. Model-based survey analysis methods were combined with small area techniques to produce county CRCS prevalence in Missouri. Adjustments based on 2010 Census population data were used to correct the bias from MO-BRFSS data, which has no weighting variable for a regular county-level survey analysis. Besides the demographic covariates, we include county attributes to improve our estimates. However, due to zero or small sample sizes for many counties in MO-BRFSS data, our attempt of including random county effect did not bring us any significant improvements in our estimates.

We classified people into 12 categories based on their age groups, gender and race. 
Ideally, finer classification with more demographic variables can provide more accurate estimates. However, our MO-BRFSS data does not support such fine categories. In general, our model based on MO-BRFSS data can provide similar but less accurate point estimates compared to CLS and spatial variation can be effectively investigated.

The estimates from CLS were treated as "true" values for comparison. However, the variance of the HT estimator was not considered. In addition, we assumed the prevalences of CRCS are the same between the year 2011 and 2012. The state-level prevalence estimates between them are very close. However, we don't know if the similarity holds at county-level.

We only studied CRCS prevalence in our paper. In general, BRFSS data contain many health-related questions so that similar studies can be conducted. However, zero or small sample sizes for some counties may still be a block when one tries to produce accurate county-level estimates. 


\section{Chapter 4}

\section{Hierarchical Models for Breast Cancer Survival in Missouri with Spatial, Age, and Stage Effects}

The breast cancer survival data in Missouri was analyzed in this chapter by considering its relationships with age, stage and county at diagnosis. A Bayesian hierarchical model was used to model the survival time, which was assumed following a Weibull distribution. The rate parameter in Weibull distribution was further modeled by age, stage and county effects. The age effect was modeled non-linearly using a one dimensional smoothing spline. The county effect was modeled by Conditional Autoregressive (CAR) distribution. Several models were studied by considering different sets of parameter combinations. 


\subsection{Introduction}

The relationship between cancer survival and individual characteristics has been mostly studied. For example, the effects of age, race, tumor size, tumor grade and disease stage were used when modeling breast cancer survival in Rosenberg et al. (2005). Those individual characteristics show significant effects when estimating breast cancer survival rates. However, some factors aside from the disease itself may also be important to the survival, such as the patient's income level, insurance status and quality of cancer treatments the patient receives. Those factors may be more or less represented by the difference among the regions in which the patient lives in. Our study is motivated by the importance of assessing small-area variation of breast cancer survival, which in turn is useful to detect if disparities exists in different areas so that proper medical and education interventions can be implemented. We used the breast cancer survival data provided by the Missouri Cancer Registry and Research Center (MCR-ARC) which maintains the state's population-based registry of all reportable tumors diagnosed among Missouri residents. These data were used to decide if there are significant differences in county-level survival rates. For our study, we selected cases diagnosed during a 10-year span from 2001 to 2010 with follow-up through 2011. Only malignant cases were selected; cases diagnosed as in situ stage were excluded. We also limited our analysis to patients whose first or only reportable primary tumor was breast cancer: women who were diagnosed with a prior non-breast tumor were excluded and survival time was measured only from the first breast cancer if multiple breast cancer were diagnosed. We included age at diagnosis, county at diagnosis and stage (localized, regional, distant and unknown/unstaged) as our variables in the analysis. We were only interested in cause-specific survival 
rates, so only deaths caused by breast cancer within our study period were treated as events, deaths due to other causes and women alive through 2011 were right censored. A total of 35,956 patients were included in our study. A Bayesian hierarchical model can be used for our survival analysis. The survival time can be modeled with a Weibull distribution and its rate parameter can be modeled with all the covariates after log transformation. The frequentist method with maximum likelihood estimates in a Weibull model can also be used, but is difficult with small sample size (Smith and Naylor, 1987). In our analysis, although we have 35,956 patients, the cell sizes would be small or even zero after classify all the patients into county-age-stage categories. A similar Bayesian hierarchical model was proposed in Dai et al. (2007), which used breast cancer survival data from Iowa Cancer Registry to study the survival rates for women over 65 years of age. The model only incorporated age at diagnosis as a linear effect together with county effects to study geographical variation of breast cancer survival. The county effects were modeled using a conditional autoregressive (CAR) model introduced in Besag (1974) and further explained in Banerjee et al. (2014).

In this chapter, we extend the model in Dai et al. (2007) to detect if breast cancer survival varies among counties in Missouri. The linear age effect might be valid in Dai et al. (2007) since they only considered patients over 65 years old. However, since we included all patients at different ages in our study, the linear assumption for age effect might not hold any more based on Rosenberg et al. (2005), Wingo et al. (1998) and Yancik et al. (2001). We proposed a new model directly extended from Dai et al. (2007) by treating age effect smoothly and non-linearly as well as including stage effects. Patients' ages were classified into 12 age groups and modeled nonparametrically using a one dimensional smoothing spline method as in Zhang et al. 
(2006). Stage at diagnosis was treated as categorical variable and the county effect retains a CAR prior. This chapter is organized as follows: Section 4.2 tells the data structure used in our analysis and introduces the models used; Section 4.3 shows the model fitting result and in the end, Section 4.4 concludes the whole chapter.

\subsection{Data and Models}

\subsubsection{Data and notation}

We had $N=35,956$ female patients in our analysis with their age, stage and county at diagnosis information included. All patients were diagnosed with invasive breast cancer.

Since we only had invasive female breast cancer cases in our analysis, the stage at diagnosis included localized, regional, distant and unknown/unstaged stages. We used three dummy variables $x_{1, i, 1}, x_{1, i, 2}$ and $x_{1, i, 3}$ to represent the four stages for the $i^{t h}$ patient. If we define $\boldsymbol{x}_{1, i}=\left(x_{1, i, 1}, x_{1, i, 2}, x_{1, i, 3}\right)^{\prime}$, then the localized stage was used as the reference stage with $\boldsymbol{x}_{1, i}=(0,0,0)^{\prime}$, while the regional, distant and unknown/unstaged stages corresponded to $(1,0,0)^{\prime},(0,1,0)^{\prime}$ and $(0,0,1)^{\prime}$, respectively.

For all the patients, ages ranged from late teens to over 90 years old. To be consistent with most SEER data base, we separate ages into 5-year intervals. There were only $1.83 \%$ (657 cases) patients less than 35 years old, which is not enough for finer classification considering different stages and counties. Thus patients aged 34 or less were combined into the first age category, followed by 35-39, 40-44, $\ldots, 80-84$ and 85 years old or over. Using the same idea of dummy variable, let 
$\boldsymbol{x}_{2, i}=\left(x_{2, i, 1}, \ldots, x_{2, t, 2}, \ldots, x_{2, i, T}\right)^{\prime}$ be an indicator variable where only $x_{2, t, 2}=1$ with rest elements equal to zero. Thus $\boldsymbol{x}_{2, i}$ will indicate patient $i$ belongs to age category $t, t=1, \ldots, T$.

The geographical effects for patient $i$ is coded in the same way. We used county at diagnosis for our analysis. There are 114 counties in Missouri plus the City of St. Louis, a county-equivalent independent city. So we used an indicator vector $\boldsymbol{x}_{3, i}=\left(x_{3, i, 1}, \ldots, x_{3, k, 2}, \ldots, x_{3, i, K}\right)^{\prime}$ with $x_{3, k, 2}=1$ to indicate the county at diagnosis for patient $i$ is the county $k$.

The variable of interest is the survival time of each patient measured in years. Let $t_{i}$ be the survival time for the $i^{\text {th }}$ patient in our data set. Since we wanted to model the cause-specific survival rates, only patients who died of breast cancer within our study time period are counted as an event in our survival analysis. The survival time for patients alive or died of other causes at the end of our study time period were treated as right censored and denoted as $c_{i}$ for the $i^{\text {th }}$ patient. Thus the data in this model were represented using pairs of random variables $\left(y_{i}, \Delta_{i}\right)$, where $y_{i}=\min \left(t_{i}, c_{i}\right)$ and $\Delta_{i}=1$ if $t_{i} \leq c_{i}, \Delta_{i}=1$ otherwise. Note that if $\Delta_{i}$ contained the censoring information of the $i^{\text {th }}$ patient.

Several figures are provided to look at the data more closely. Figure 4.1 shows the age distribution for 4 different stages. Patients' ages were distributed fairly similarly across localized, regional and distant stages, with peaks around 50 to 64 years old, and heavy tail on the right for older women. The unknown/unstaged stages were mostly concentrated around older ages, especially 85 years old and over. As indicated by the range of the y-axis for each sub-figure, most patients were diagnosed with localized stage, followed by regional and then distant stages. Conversely, Figure 4.2 shows the 


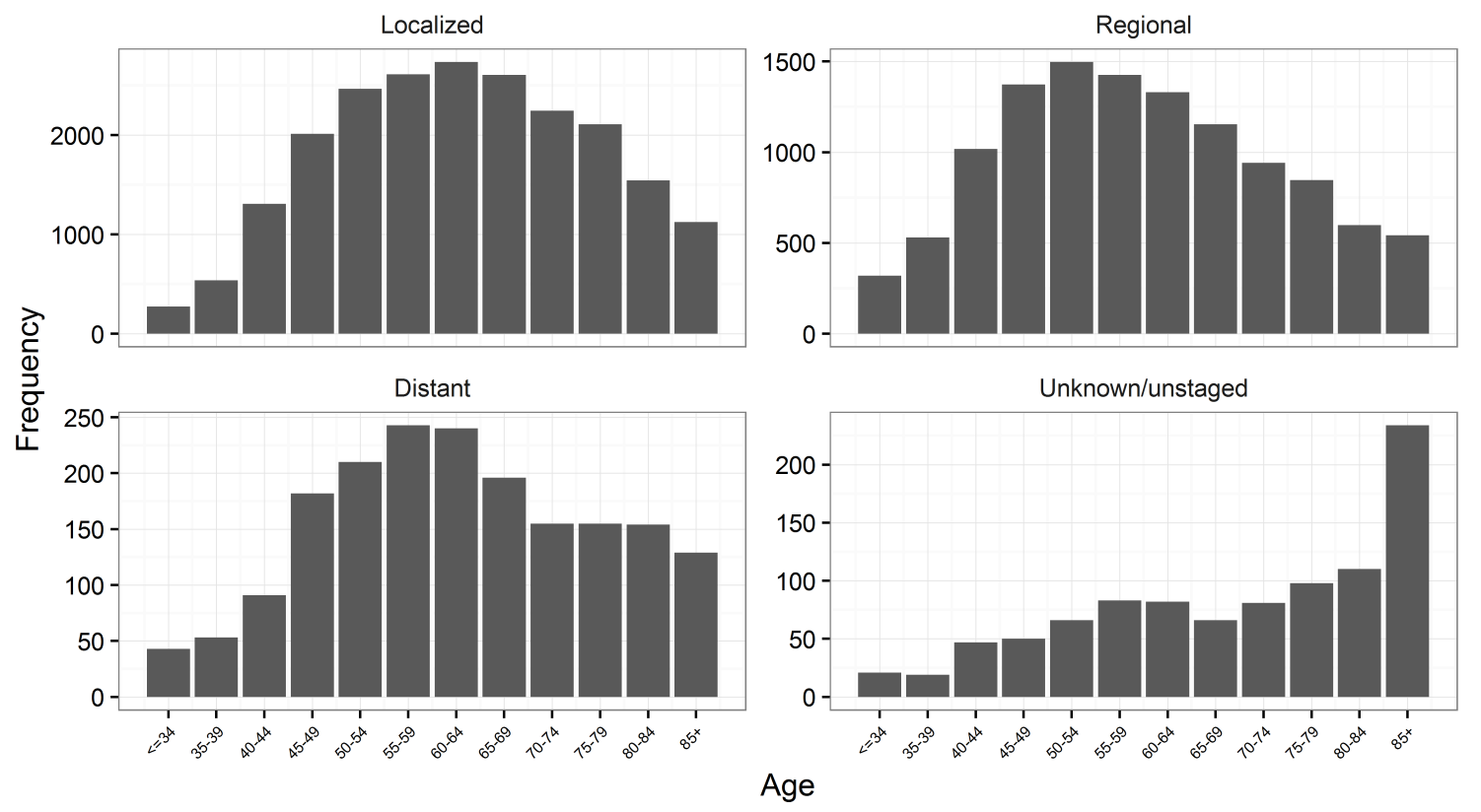

Figure 4.1: Age Distribution Across Stages.

stage distribution for each age group in percentage. For each age group along the $\mathrm{x}$-axis, there are four points representing the proportion of the corresponding four stages which sum to $100 \%$. For women less than 35 years old, a relatively larger proportion of these patients were diagnosed with distant stage compared to women aged between 35 and 69 . For the localized stage cases, the proportion was increasing when we are looking at older women. However, there was a drop for women over 85 years old. In addition, Figure 4.3 shows the proportion of women who died of breast cancer for each group. We noticed that young women (less than 45) and older women (75 and over) generally had worse survival than middle-aged women. The U-shape relationship between the death proportion and ages also suggested the non-linear relationship between survival time and age. In the end, we examined the cancer stage distribution across counties in Missouri with Figure 4.4. We only included localized, 
regional and distant stages for the maps. We noticed that the patients' stages were not uniformly distributed across Missouri.

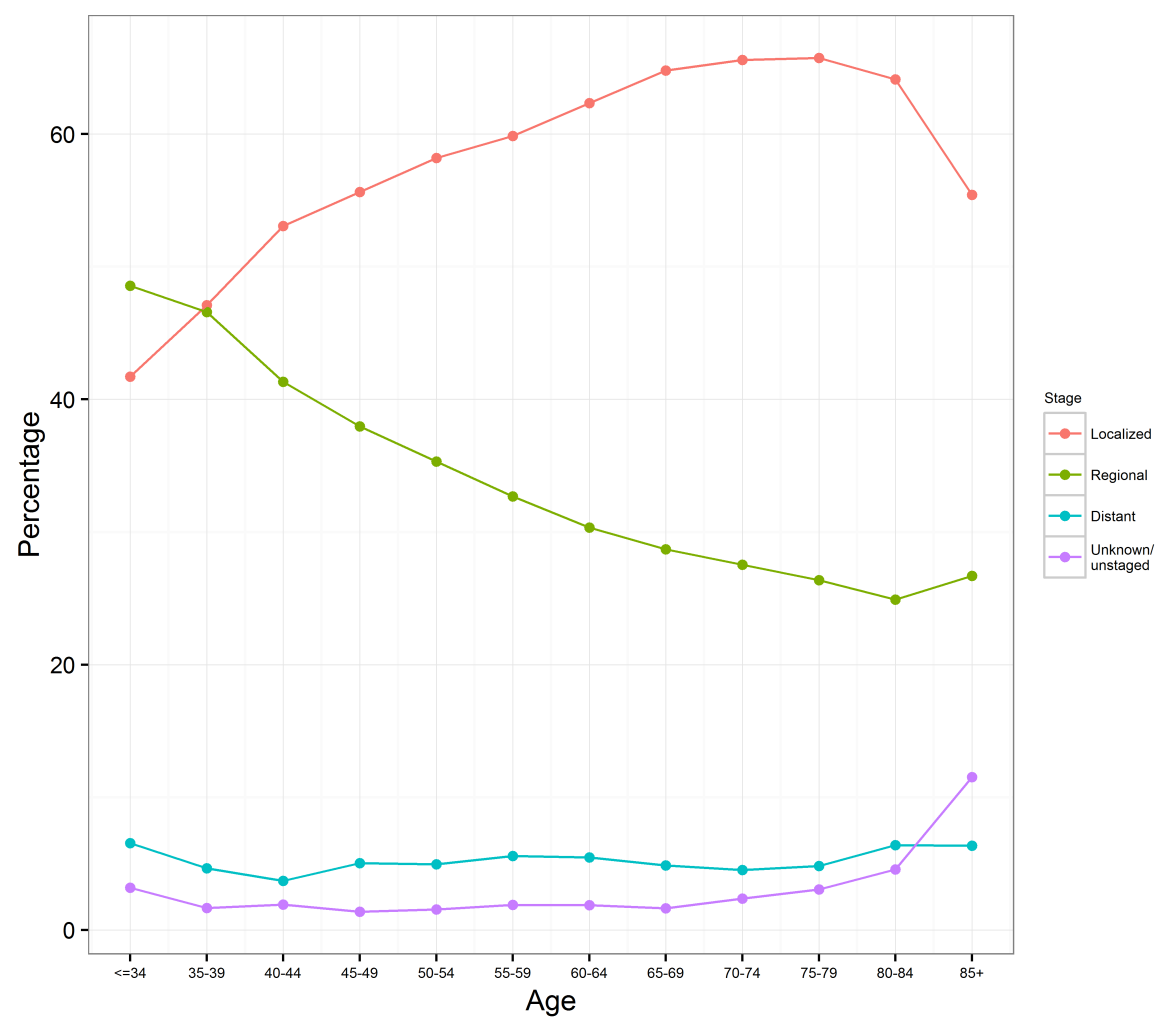

Figure 4.2: Stage Percentages for All Age Groups. For each age group, the percentages for four stages sum to $100 \%$.

\subsubsection{Models}

In this section, we used Bayesian hierarchical models to fit our survival data, with age, stage and county effects. 


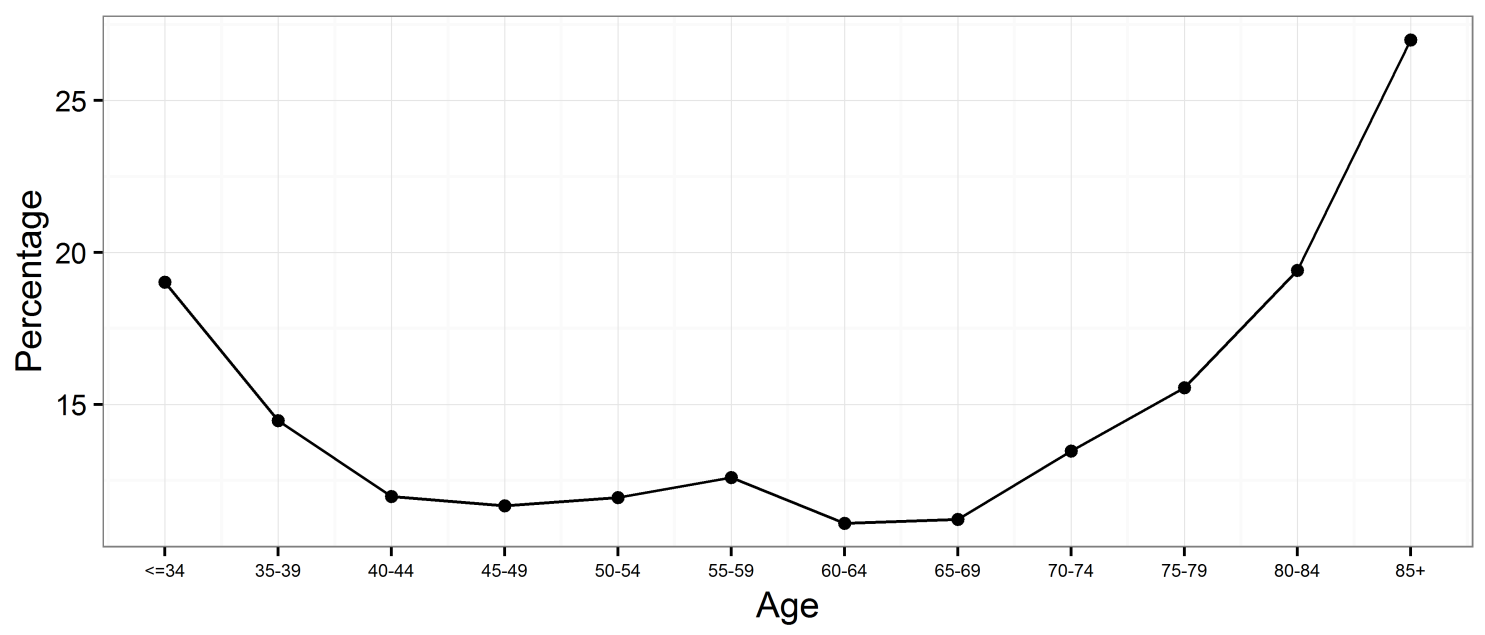

Figure 4.3: Proportion of $\Delta=1$. The percentage represents the proportion of patients who died of breast cancer within the study period.

(a) Localized
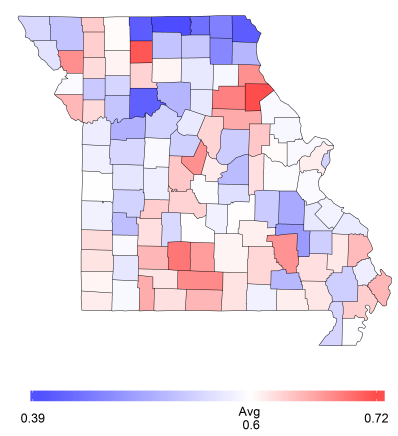

(b) Regional
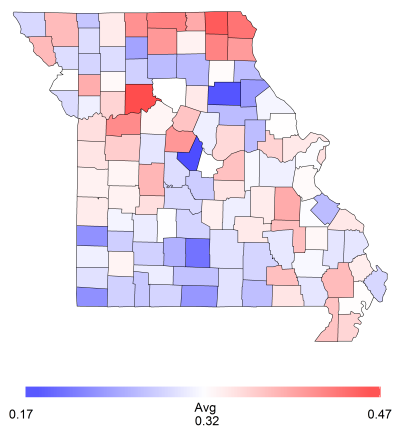

(c) Distant
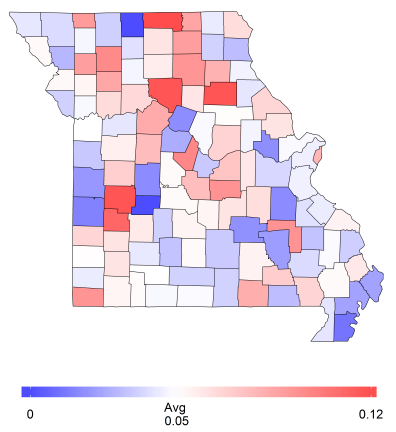

Figure 4.4: Proportion of Localized, Regional and Distant Staged Patients within Each County. White represents the average proportion in Missouri for the corresponding stages. 


\section{Likelihood function}

We assumed the survival time $t_{i}$ for the $i^{\text {th }}$ patient follows a Weibull distribution $W\left(\alpha, \lambda_{i}\right)$, whose density and survival function are given by

$$
f\left(t \mid \alpha, \lambda_{i}\right)=\alpha \lambda_{i} t^{\alpha-1} \exp \left(-\lambda_{i} t^{\alpha}\right)
$$

and

$$
S\left(t \mid \alpha, \lambda_{i}\right)=\exp \left(-\lambda_{i} t^{\alpha}\right), \quad t>0
$$

respectively. The exact survival time $t_{i}$ will be observed only if $\Delta_{i}=1$. As convention in generalized Weibull regression, we used covariates to model the rate parameter $\lambda_{i}$ after logarithm transformation. Let $v_{i}=\log \left(\lambda_{i}\right)$, then the survival function can be written as

$$
f\left(t \mid \alpha, v_{i}\right)=\alpha t^{\alpha-1} \exp \left[v_{i}-\exp \left(v_{i}\right) t^{\alpha}\right]
$$

Based on $(\boldsymbol{y}, \boldsymbol{\Delta})$, the likelihood function of $\boldsymbol{v}=\left(v_{1}, \ldots, v_{N}\right)^{\prime}$ and $\alpha$ is

$$
\begin{aligned}
L(\alpha, \boldsymbol{v} \mid \boldsymbol{y}, \boldsymbol{\Delta}) & =\prod_{i=1}^{i=N}\left\{\left[f\left(y_{i} \mid \alpha, v_{i}\right)\right]^{\Delta_{i}} \times\left[S\left(y_{i} \mid \alpha, v_{i}\right)\right]^{1-\Delta_{i}}\right\} \\
& =\exp \left\{\sum_{i=1}^{N}\left(\Delta_{i}\left[\log (\alpha)+v_{i}+\alpha \log \left(y_{i}\right)-\log \left(y_{i}\right)\right]-e^{v_{i}} y_{i}^{\alpha}\right)\right\}
\end{aligned}
$$

\section{Models for the linear predictor}

For transformed rates $\boldsymbol{v}$, a linear mixed model was used with stage effects $\boldsymbol{\beta}=$ $\left(\beta_{1}, \ldots, \beta_{S}\right)^{\prime}$, age effects $\boldsymbol{\theta}=\left(\theta_{1}, \ldots, \theta_{T}\right)^{\prime}$ and county effects $\boldsymbol{w}=\left(w_{1}, \ldots, w_{k}\right)^{\prime}$, where

$S=3, T=12$ and $K=115$ in our study. Several models were considered in our 
analysis. Using the variable notation in section $4.2 .1, \boldsymbol{x}_{1, i}, \boldsymbol{x}_{2, i}$ and $\boldsymbol{x}_{3, i}$ indicate the cancer stage, age group and county at diagnosis for patient $i$, respectively. Then for patient $i$, the product $\boldsymbol{x}_{1, i}^{\prime} \boldsymbol{\beta}, \boldsymbol{x}_{2, i}^{\prime} \boldsymbol{\theta}$ and $\boldsymbol{x}_{3, i}^{\prime} \boldsymbol{w}$ represent the corresponding stage, age and county effects. For example, if patient $i$ is diagnosed as stage $s \in(1,2,3)$, belongs to age category $t \in(1, \ldots, T)$ and county $k \in(1, \ldots, K)$, then $\boldsymbol{x}_{1, i}^{\prime} \boldsymbol{\beta}=\beta_{s}, \boldsymbol{x}_{2, i}^{\prime} \boldsymbol{\theta}=\theta_{t}$ and $\boldsymbol{x}_{3, i}^{\prime} \boldsymbol{w}=w_{k}$. With these notations, we model $\boldsymbol{v}$ as

$$
v_{i}=\beta_{0}+\boldsymbol{x}_{1, i}^{\prime} \boldsymbol{\beta}+\boldsymbol{x}_{2, i}^{\prime} \boldsymbol{\theta}+\boldsymbol{x}_{3, i}^{\prime} \boldsymbol{w}+\epsilon_{i}, \quad i=1, \ldots, N,
$$

where $\beta_{0}$ is an intercept term and $\epsilon_{i}$ captures additional patient-level errors information not included in our model. If we define $\boldsymbol{X}_{1}=\left(\boldsymbol{x}_{1,1}, \ldots, \boldsymbol{x}_{1, N}\right)^{\prime}, \boldsymbol{X}_{2}=$ $\left(\boldsymbol{x}_{2,1}, \ldots, \boldsymbol{x}_{2, N}\right)^{\prime}, \boldsymbol{X}_{3}=\left(\boldsymbol{x}_{3,1}, \ldots, \boldsymbol{x}_{3, N}\right)^{\prime}$ to be the design matrix for all observations to indicate the corresponding age, stage and counties, and $\boldsymbol{\epsilon}=\left(\epsilon_{1}, \ldots, \epsilon_{N}\right), \mathbf{1}_{N}=$ $(1, \ldots, 1)_{N \times 1}^{\prime}$, then model (4.6) can be rewritten in matrix from,

$$
\boldsymbol{v}=\beta_{0} \mathbf{1}_{N}+\boldsymbol{X}_{1} \boldsymbol{\beta}+\boldsymbol{X}_{2} \boldsymbol{\theta}+\boldsymbol{X}_{3} \boldsymbol{w}+\boldsymbol{\epsilon} .
$$

The unstructured error term $\boldsymbol{\epsilon}$ follows a normal prior $\boldsymbol{\epsilon} \sim N_{N}\left(\mathbf{0}, \delta_{0} \boldsymbol{I}\right)$, where $\delta_{0}$ is the variance parameter. If we collect all the covariates and parameters above by defining $\boldsymbol{X}_{N \times(1+S+T+K)}=\left(\mathbf{1}_{N}, \boldsymbol{X}_{1}, \boldsymbol{X}_{2}, \boldsymbol{X}_{3}\right)$ and $\boldsymbol{\psi}_{(1+S+T+K) \times 1}=\left(\beta_{0}, \boldsymbol{\beta}^{\prime}, \boldsymbol{\theta}^{\prime}, \boldsymbol{w}^{\prime}\right)^{\prime}$, then the distribution of $\boldsymbol{v}$ is

$$
\left[\boldsymbol{v} \mid \boldsymbol{\psi}, \delta_{0}\right] \sim N_{N}\left(\boldsymbol{X} \boldsymbol{\psi}, \delta_{0} \boldsymbol{I}\right)
$$

The model above included all covariates in our data, however one may wonder if all covariates are actually needed. Different structures of $\boldsymbol{X}$ and $\boldsymbol{\psi}$ can be constructed based on the covariates included. In our analysis, we considered the following models: 
- Model 1: Linear age effect + spatial effect, as used in Dai et al. (2007):

$$
\boldsymbol{X}_{N \times(2+K)}=\left(\mathbf{1}_{N}, \boldsymbol{x}_{\text {age }}, \boldsymbol{X}_{3}\right), \quad \boldsymbol{\psi}_{(2+K) \times 1}=\left(\beta_{0}, \gamma, \boldsymbol{w}^{\prime}\right)^{\prime},
$$

where $\boldsymbol{x}_{\text {age }}$ is an $N \times 1$ vector contains the standardized ages for all patients, which treated as a continuous variable and $\gamma$ is the slope parameter.

- Model 2: stage effect + linear age effect + spatial effect :

$$
\boldsymbol{X}_{N \times(2+S+K)}=\left(\mathbf{1}_{N}, \boldsymbol{X}_{1}, \boldsymbol{x}_{a g e}, \boldsymbol{X}_{3}\right), \quad \boldsymbol{\psi}_{(2+S+K) \times 1}=\left(\beta_{0}, \boldsymbol{\beta}^{\prime}, \gamma, \boldsymbol{w}^{\prime}\right)^{\prime},
$$

- Model 3: stage effect + categorical age effect + spatial effect:

$$
\boldsymbol{X}_{N \times(1+S+T+K)}=\left(\mathbf{1}_{N}, \boldsymbol{X}_{1}, \boldsymbol{X}_{2}, \boldsymbol{X}_{3}\right), \quad \boldsymbol{\psi}_{(1+S+T+K) \times 1}=\left(\beta_{0}, \boldsymbol{\beta}^{\prime}, \boldsymbol{\theta}^{\prime}, \boldsymbol{w}^{\prime}\right)^{\prime},
$$

which is our full model above.

- Model 4: stage effect + categorical age effect:

$$
\boldsymbol{X}_{N \times(1+S+T)}=\left(\mathbf{1}_{N}, \boldsymbol{X}_{1}, \boldsymbol{X}_{2}\right), \quad \boldsymbol{\psi}_{(1+S+T) \times 1}=\left(\beta_{0}, \boldsymbol{\beta}^{\prime}, \boldsymbol{\theta}^{\prime}\right)^{\prime},
$$

where we removed the spatial effect.

- Model 5: stage effect + categorical age effect + spatial effect with stage-specific shape parameter $\boldsymbol{\alpha}=\left(\alpha_{1}, \ldots, \alpha_{4}\right)^{\prime}$. The covariates $\boldsymbol{X}$ and parameter $\boldsymbol{\psi}$ are the same as those in Model 3, but the shape parameter varies in (4.3) according to the stage which the patient was diagnosed with. To be specific, let $\boldsymbol{X}_{\alpha}$ be 
an $N \times 4$ indicator matrix to indicate the stages for all patients. Then the likelihood function (4.5) will be

$$
\begin{aligned}
& L(\boldsymbol{\alpha}, \boldsymbol{v} \mid \boldsymbol{y}, \boldsymbol{\Delta}) \\
& =\exp \left\{\sum_{i=1}^{N}\left(\Delta_{i}\left[\log \left(\boldsymbol{x}_{\alpha, i} \boldsymbol{\alpha}\right)+v_{i}+\boldsymbol{x}_{\alpha, i} \boldsymbol{\alpha} \log \left(y_{i}\right)-\log \left(y_{i}\right)\right]-e^{v_{i}} y_{i}^{\boldsymbol{x}_{\alpha, i} \boldsymbol{\alpha}}\right)\right\},
\end{aligned}
$$

where $\boldsymbol{x}_{\alpha, i}$ is the $i^{\text {th }}$ row of $\boldsymbol{X}_{\alpha}$.

\section{Priors for the parameters of the linear predictors}

Different priors were given to parameters of the linear predictors. For the intercept $\beta_{0}$ and stage effect $\boldsymbol{\beta}$, vague normal priors were used:

$$
\beta_{0} \sim N(0, \tau), \quad \boldsymbol{\beta} \sim N_{3}(\mathbf{0}, \tau \boldsymbol{I})
$$

where $\boldsymbol{I}$ is identity matrix. In Models 1 and 2 where a linear age effect presented, we also use $N(0, \tau)$ as the prior for the slope parameter $\gamma$.

We used a CAR model for the spatial effect $\boldsymbol{w}=\left(w_{1}, \ldots, w_{K}\right)^{\prime}$ in our analysis, as described in Chapter 3.2.4. With the notations used in this Chapter, let $j \sim k$ to denote that county $j$ is adjacent to $k$ and $\boldsymbol{C}=\left(c_{k j}\right)_{K \times K}$ be the adjacency matrix. Then the CAR prior for $\boldsymbol{w}$ is

$$
[\boldsymbol{w} \mid \delta, \rho] \sim N_{K}\left(\mathbf{0}, \delta(\boldsymbol{I}-\rho \boldsymbol{C})^{-1}\right)
$$

where $\delta>0$ specifies the conditional variance, $\sum_{j \sim i} w_{j}$ is the sum of neighboring variables around county $k$, and $\rho$ specifies the strength of the relationship between 
county $k$ and its neighbors. Restrictions on $\rho$ is the same as (3.17).

Instead of assuming a linear trend in age, we would like to identify a smooth non-linear or non-parametric trend, which was used in Models 3, 4 and 5. In this study, a one dimensional smoothing spline model was used as the prior distribution for the categorical age effects, which we briefly review here. Consider a one variable regression on data $\boldsymbol{Y}=\left(y_{1}, \ldots, y_{T}\right)^{\prime}$ :

$$
y_{i}=\theta\left(t_{i}\right)+e_{i}, \quad e_{1}, \ldots, e_{T} \stackrel{i . i . d}{\sim} N\left(0, \tau_{0}\right)
$$

where $\tau_{0}$ is the error variance. The solution to a $q^{\text {th }}$ order smoothing spline is the solution to the minimization problem

$$
\hat{\boldsymbol{\theta}}=\arg \min _{g \in \mathcal{G}}\left[\sum_{i=1}^{T} y_{i}-g\left(t_{i}\right)^{2}+\eta \int g^{(q)}(t)^{2} \mathrm{~d} t\right],
$$

where $\mathcal{G}$ is the class of functions with $q$ derivatives. There exist correspondence between the smoothing spline problems and Bayesian estimation problems as shown in Kimeldorf and Wahba (1970). To be specific, if we assign $\boldsymbol{\theta}$ a prior distribution

$$
\left[\boldsymbol{\theta} \mid \tau_{1}\right] \propto \tau_{1}^{-(T-p) / 2} \exp \left(-\frac{1}{2 \tau_{1}} \boldsymbol{\theta}^{\prime} \boldsymbol{A} \boldsymbol{\theta}\right)
$$

where $\boldsymbol{A}$ is a $T \times T$ non-negative matrix of $\operatorname{rank} T-q$, and $\tau_{1}$ is a variance component, then the posterior distribution of $\boldsymbol{\theta}$ given $\left(\tau_{0}, \tau_{1}, \boldsymbol{Y}\right)$ is $N\left(\boldsymbol{S}_{\eta} \boldsymbol{Y}, \tau_{1} \boldsymbol{S}_{\eta}\right)$, where $\eta=\tau_{0} / \tau_{1}$ and $\boldsymbol{S}_{\eta}=(\boldsymbol{I}+\eta \boldsymbol{A})^{-1}$ is the smoother matrix. It is shown that $\boldsymbol{A}$ can be chosen as an intrinsic autoregressive precision matrix of order $q$, IAR(q), so that the posterior mean $\boldsymbol{S}_{\eta} \boldsymbol{Y}$ for fixed $(\eta, \boldsymbol{Y})$ is the solution to the $q^{\text {th }}$ order smoothing spline problem. 
An IAR(2) process was used in Zhang et al. (2006) to get smoothed estimates of cancer incidence and mortality rates over time. We used the same method to model the age effects in our analysis smoothly. Since our age groups could be treated equally spaced except for the first and last ones, the elements of an IAR(q) precision matrix $\boldsymbol{A}_{q}=\left(a_{i j}\right)_{T \times T}$, except the edge effects, are defined as

$$
a_{i j}= \begin{cases}(-1)^{|i-j|}\left(\begin{array}{c}
2 p \\
p-|i-j|
\end{array}\right), & \text { if }|i-j| \leq q \\
0, & \text { otherwise }\end{cases}
$$

In our study, the same second order smoothing matrix was used with $q=2$. Then the smoothing matrix is

$$
\boldsymbol{A}_{2}=\left(\begin{array}{ccccccccccc}
1 & -2 & 1 & 0 & 0 & \cdots & 0 & 0 & 0 & 0 & 0 \\
-2 & 5 & -4 & 1 & 0 & \cdots & 0 & 0 & 0 & 0 & 0 \\
1 & -4 & 6 & -4 & 1 & \cdots & 0 & 0 & 0 & 0 & 0 \\
\vdots & \vdots & \vdots & \vdots & \vdots & \ddots & \vdots & \vdots & \vdots & \vdots & \vdots \\
0 & 0 & 0 & 0 & 0 & \cdots & 1 & -4 & 6 & -4 & 1 \\
0 & 0 & 0 & 0 & 0 & \cdots & 0 & 1 & -4 & 5 & 2 \\
0 & 0 & 0 & 0 & 0 & \cdots & 0 & 0 & 1 & -2 & 1
\end{array}\right)_{T \times T}
$$

Note that the rank of $\boldsymbol{A}_{2}$ is $T-2$. In our analysis, $T=12$. Assuming (4.17) and (4.19) as the prior for $\boldsymbol{\theta}$ is equivalent to applying a non-parametric regression. In order to properly identify $\beta_{0}$ and $\boldsymbol{\theta}$, we added sum-to-zero restrictions to $\boldsymbol{\theta}$ by subtracting its mean, and adjusting the overall intercept $\beta_{0}$ by adding the same quantity. 


\section{Priors for the remaining parameters}

Since the shape parameter $\alpha$ in the Weibull model is often quite stable, we assigned a gamma prior for it:

$$
\alpha \sim \operatorname{Gamma}\left(a_{\alpha}, b_{\alpha}\right) .
$$

The same prior distributions were used when we have different $\boldsymbol{\alpha}$ in Model 5. In this paper, an $x$ random variable following a $\operatorname{Gamma}(a, b)$ distribution has the density function

$$
f(x)=\frac{b^{a}}{\Gamma(a)} x^{a-1} \exp (-b x), \quad \text { for } x>0 .
$$

The gamma distributions was chosen because of its flexibility and convenience.

The variance parameter $\tau_{1}$ in (4.17) was given an Inverse-Gamma $\left(a_{1}, b_{1}\right)$ prior distribution with density proportional to

$$
f\left(\tau_{1}\right) \propto \frac{1}{\tau_{1}^{a_{1}-1}} \exp \left(-\frac{b_{1}}{\tau_{1}}\right), \quad \text { for } \tau_{1}>0 .
$$

The parameter $\rho$ controls the strength of spatial association and is restricted in (3.17). Thus we used a uniform prior distribution

$$
\rho \sim \operatorname{Unif}\left(\frac{1}{\min \nu_{i}}, \frac{1}{\max \nu_{i}}\right) .
$$

To complete our hierarchical model, we still need a prior on the variance parameter $\delta_{0}$ in (4.8) and $\delta$ in (4.14). We used the same strategy as in Chapter 3.2.5. To be specific, an inverse gamma type prior on $\delta_{0}$ :

$$
\left[\delta_{0}\right] \propto \frac{1}{\delta_{0}^{a_{0}+1}} \exp \left(\frac{b_{0}}{\delta_{0}}\right)
$$


and linking $\delta_{0}$ with $\delta$ by the noise-to-signal ratio $\eta=\delta_{0} / \delta$. A scaled Pareto prior on $\eta$ is used

$$
[\eta \mid a]=\frac{a}{(a+\eta)^{2}}, \quad \eta>0
$$

with $a=1$. One benefit of using this set up is that the prior specifications for $\delta_{0}$ and $\delta$ is equivalent to assume

$$
\frac{\delta}{\delta_{0}+\delta} \sim \operatorname{Unif}(0,1)
$$

\subsection{Model Comparison and Results}

\subsubsection{Computation}

An Markov Chain Monte Carlo (MCMC) algorithm was used to generate the posterior distributions of parameters in our model. Based on (4.5), (4.8), (4.13), (4.14), (4.17), (4.20), (4.22), (4.24) and (4.25), the joint posterior density of $\left(\boldsymbol{v}, \alpha, \boldsymbol{\psi}, \delta_{0}, \rho, \eta\right)$ given $(\boldsymbol{y}, \boldsymbol{\Delta})$ is

$$
\left[\boldsymbol{v}, \alpha, \boldsymbol{\psi}, \delta_{0}, \rho, \eta \mid \boldsymbol{y}, \boldsymbol{\Delta}\right] \propto L(\alpha, \boldsymbol{v} \mid \boldsymbol{y}, \boldsymbol{\Delta})\left[\boldsymbol{v} \mid \boldsymbol{\psi}, \delta_{0}\right]\left[\beta_{0}\right][\boldsymbol{\beta}]\left[\boldsymbol{w} \mid \delta_{0}, \rho, \delta\right]\left[\delta_{0}\right][\eta][\alpha]
$$

where $\boldsymbol{\psi}$ included parameters for the linear predictors, which were different across Models 1 to 5. A Gibbs sampling algorithm was implemented in $\mathrm{R}$ and $\mathrm{C}++$ with the full conditional densities derived in Appendix B.3. The conditional posterior distributions for $\boldsymbol{\theta}, \delta_{0}$ and $\tau_{1}$ are multivariate normal and inverse-gamma distributions, which could be sampled directly. For all the other parameters, Adaptive Rejection Metropolis Sampling (ARMS) introduced in Gilks et al. (1995) was used to get the 
posterior samples via a $\mathrm{C}++$. For the Bayesian computation, the following hyper parameter values were used:

$$
a_{\alpha}=b_{\alpha}=2, \quad a_{0}=b_{0}=a_{1}=b_{1}=1, \quad \tau=1000
$$

\subsubsection{Model comparison}

All five models in Section 4.2.2 were fitted. The posterior analysis was based on 20,000 posterior samples after discarding the first 10,000 iterations. The DIC (Chapter 2.3.3) was used to compare Model 1 to 5 to select the best one. Table 4.1 summarizes the result. The column "Dim." shows the dimension of $\boldsymbol{\psi}$ and $p_{D}$ is the number of effective parameters. Model 3 has the smallest DIC which suggests the best fit. Smaller DIC for Model 2 compared to Models 1 indicates the importance of including stage information. The comparison between Model 3 and Model 2 shows the huge improvement by treating age effect non-linearly. Model 4 omits the spatial effect and has worse fit than Model 3, which suggests it is important to keep the spatial term. The increment in DIC from Model 3 to Model 5 suggests that there is no need of having different shape parameter $\alpha$ s for different stages.

Table 4.1: DIC for competing survival models

\begin{tabular}{lccc}
\hline Model & Dim. & $P_{D}$ & DIC \\
\hline Model 1 & 117 & 3425.970 & 44456.70 \\
Model 2 & 120 & 2207.593 & 38762.35 \\
Model 3 & 131 & 2211.672 & 38507.54 \\
Model 4 & 16 & 2221.050 & 38540.64 \\
Model 5 & 134 & 1835.766 & 38681.68 \\
\hline
\end{tabular}




\subsubsection{Results}

We used posterior samples from Model 3 for the following analysis since it provided the best fit with the smallest DIC.

\section{Parameter estimation and interpretation}

Table 4.2 summarizes the posterior means and standard deviations for some selected parameters of interest. Some selected trace plots and histograms based on those posterior samples are shown in Figure 4.5 and Figure 4.6. From both figures we see no divergence issues and the mixing is quite good. We now examine some variables' partial effects given all the other fixed. For example, when considering stage effects $\boldsymbol{\beta}=\left(\beta_{1}, \beta_{2}, \beta_{3}\right)$ which stands for the regional, distant and unknown/unstaged stages comparing to the reference (localized) stage, we notice that more advanced stages have negative effect on patient's survival time (recall the survival function in (4.2)). The shape parameter $\alpha$ is estimated to be close to 1 , which indicate the Weibull distribution is acting similarly to the exponential distribution. The unstructured error variance $\delta_{0}$ dominates the structured spatial variance $\delta_{1}$ (posterior means of 0.615 vs 0.016 ), indicating the main source of variation comes from random errors. The estimated $\rho$ is skewed to its right boundary which shows positive spatial dependence. Considering the age effects by the estimates of $\boldsymbol{\theta}$, we noticed that from $\theta_{1}$ to $\theta_{12}$ their values first decreased then increased with the change point at $\theta_{4}$, which stands for women aged 45 to 49 years old. This indicates younger and older women have relatively worse breast cancer survival compared to middle-aged women. 


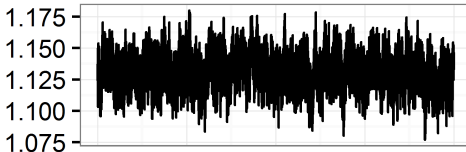

$\delta_{0}$

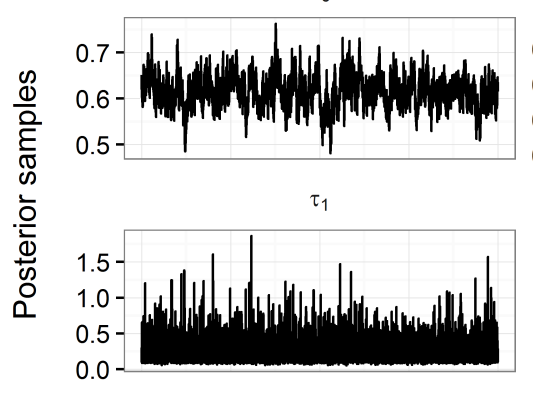

$V_{1000}$

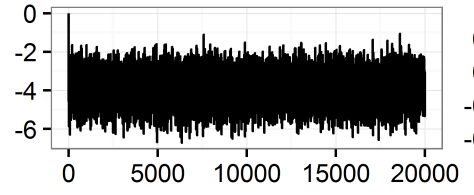

$\beta_{1}$

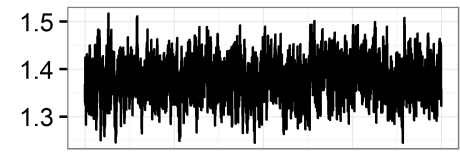

$\delta_{1}$

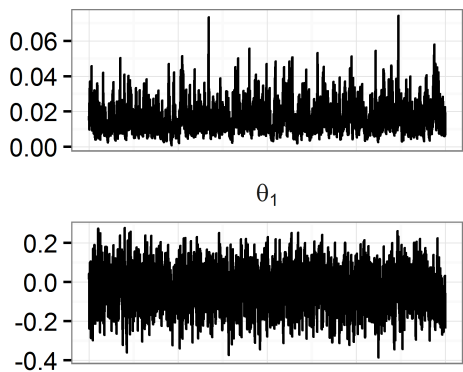

$\mathrm{w}_{2}$

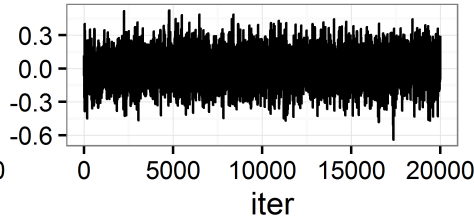

$\beta_{2}$

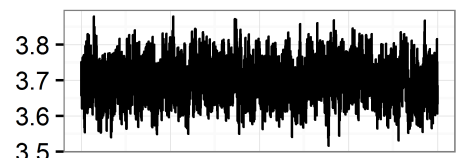

$\rho$

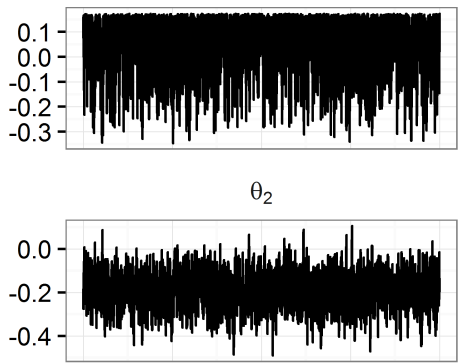

deviance

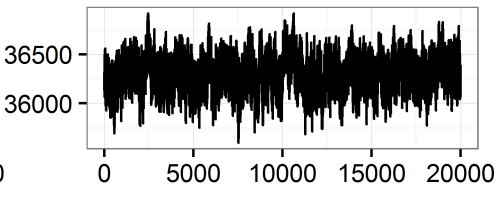

Figure 4.5: Trace Plots of Some Selected Parameters of Interest. We include the shape parameter $(\alpha)$ for the Weibull distribution, two examples $\left(\beta_{1}, \beta_{2}\right)$ for stage effect, all variance parameters $\left(\delta_{0}, \delta_{1}, \tau_{1}\right)$, spatial correlation parameters $(\rho)$, two examples $\left(\theta_{1}, \theta_{2}\right)$ for age effect, one example $\left(v_{1000}\right)$ for the log-link quantity $\boldsymbol{v}$, one example $\left(w_{2}\right)$ for spatial effect and the deviance of the model fitted. The remaining parameters had similar plots but are not shown for brevity. 
$\alpha$

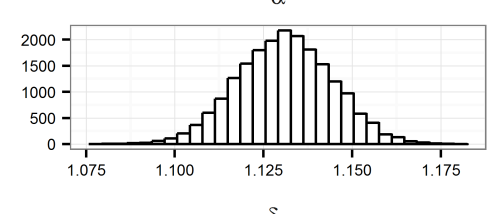

$\delta_{0}$
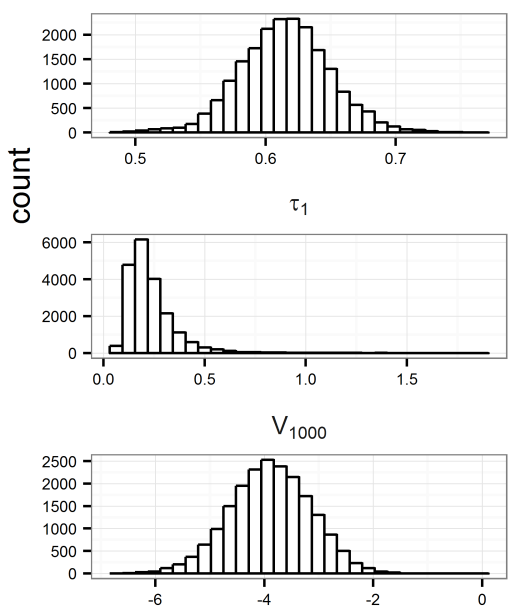

$\beta_{1}$

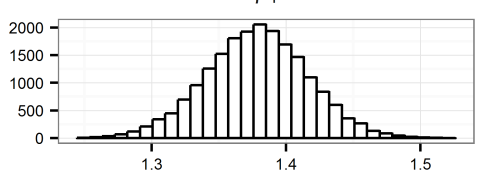

$\delta_{1}$
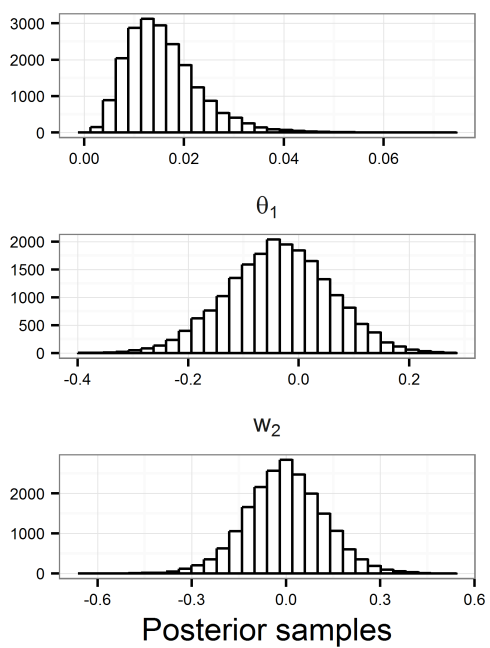

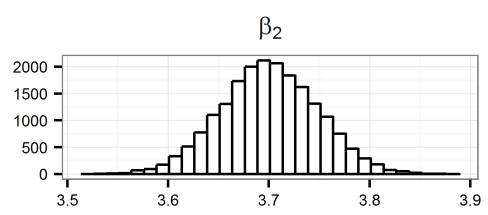

$\rho$
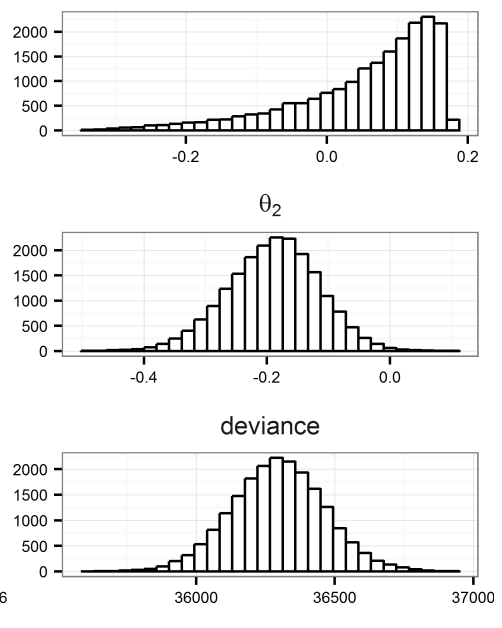

Figure 4.6: Posterior Histograms of the Parameters as in Figure 4.5. 
Table 4.2: Posterior Means and Standard Deviations (SD) for Selected Parameters.

\begin{tabular}{ccc|ccc}
\hline & Mean & SD & & Mean & SD \\
\hline$\beta_{0}$ & -5.055 & 0.046 & $\theta_{1}$ & -0.036 & 0.092 \\
$\beta_{1}$ & 1.378 & 0.038 & $\theta_{2}$ & -0.190 & 0.074 \\
$\beta_{2}$ & 3.700 & 0.048 & $\theta_{3}$ & -0.321 & 0.055 \\
$\beta_{3}$ & 2.591 & 0.064 & $\theta_{4}$ & -0.349 & 0.050 \\
$\alpha$ & 1.131 & 0.013 & $\theta_{5}$ & -0.268 & 0.046 \\
$\delta_{0}$ & 0.615 & 0.035 & $\theta_{6}$ & -0.214 & 0.044 \\
$\delta_{1}$ & 0.016 & 0.007 & $\theta_{7}$ & -0.283 & 0.047 \\
$\rho$ & 0.055 & 0.101 & $\theta_{8}$ & -0.221 & 0.046 \\
$\tau_{1}$ & 0.231 & 0.118 & $\theta_{9}$ & 0.031 & 0.047 \\
& & & $\theta_{10}$ & 0.274 & 0.047 \\
& & & $\theta_{11}$ & 0.548 & 0.051 \\
& & & $\theta_{12}$ & 1.028 & 0.049 \\
\hline
\end{tabular}

\section{Spatial effects}

In Table 2.3, the larger DIC for Model 4 compared to Model 3 suggests including the random county (spatial) effect improves our model fitting result. The spatial effects can be treated as structured errors while $\boldsymbol{\epsilon}$ are unstructured. Recall in (4.26) we assumed a uniform prior on the proportion of spatial variance relative to the whole variance arising in the second stage. The posterior means and standard deviations of this proportion are 0.025 and 0.011 , which suggest the spatial variance only took part in around $2.5 \%$ in terms of the whole variation. Although the strength of spatial correlation parameter $\rho$ has a skewed posterior distribution to its right boundary in Figure 4.6, which shows strong positive spatial correlation, the overall effect of the spatial term is weak when other variables (age and stage) are presented. Figure 4.7 shows the posterior means of the spatial effect. As only for these detected weak spatial effects, the southeast part shows relatively worse breast cancer survival given the same stage and age. 


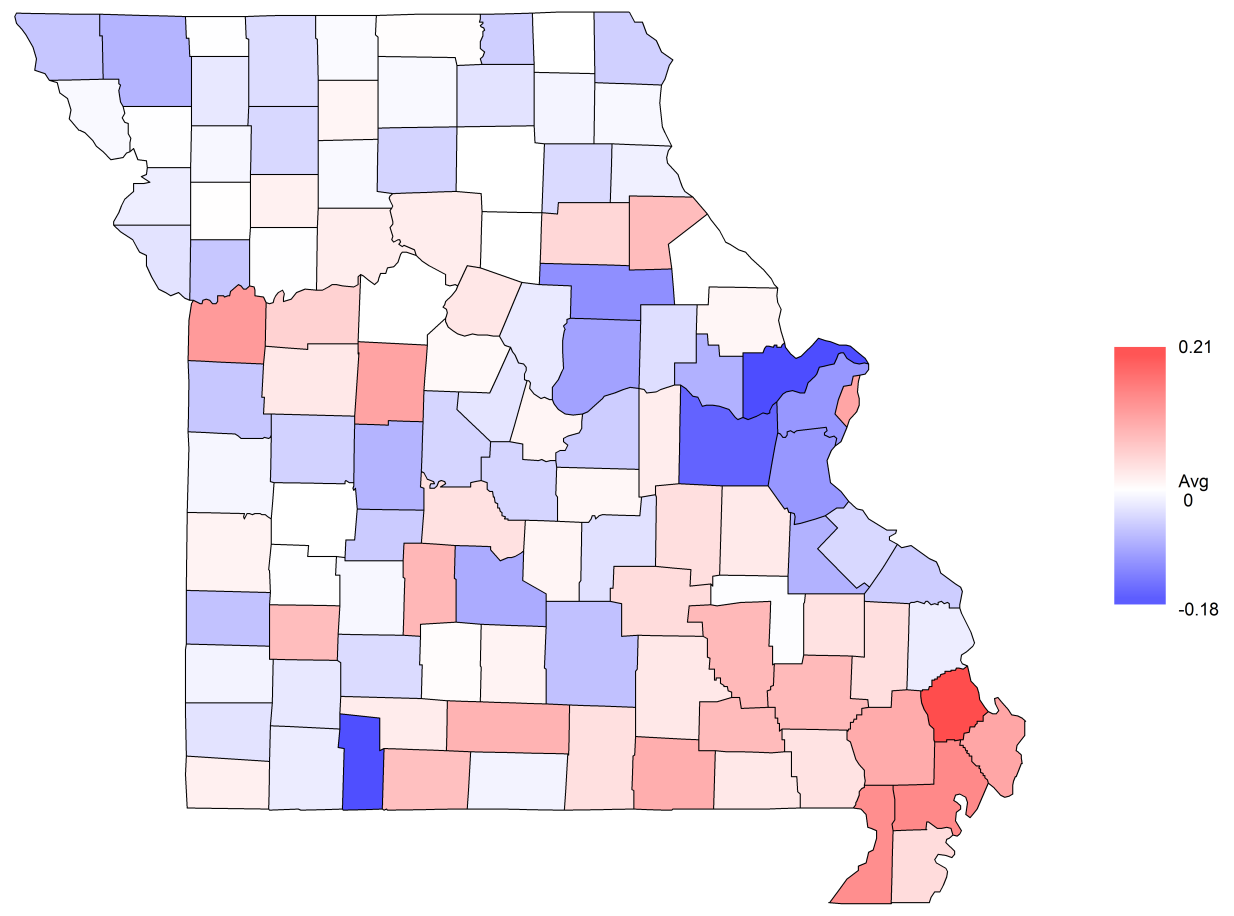

Figure 4.7: Posterior Means of Spatial Effects. White represents the average effect over all counties. Large values correspond to worse survival. 


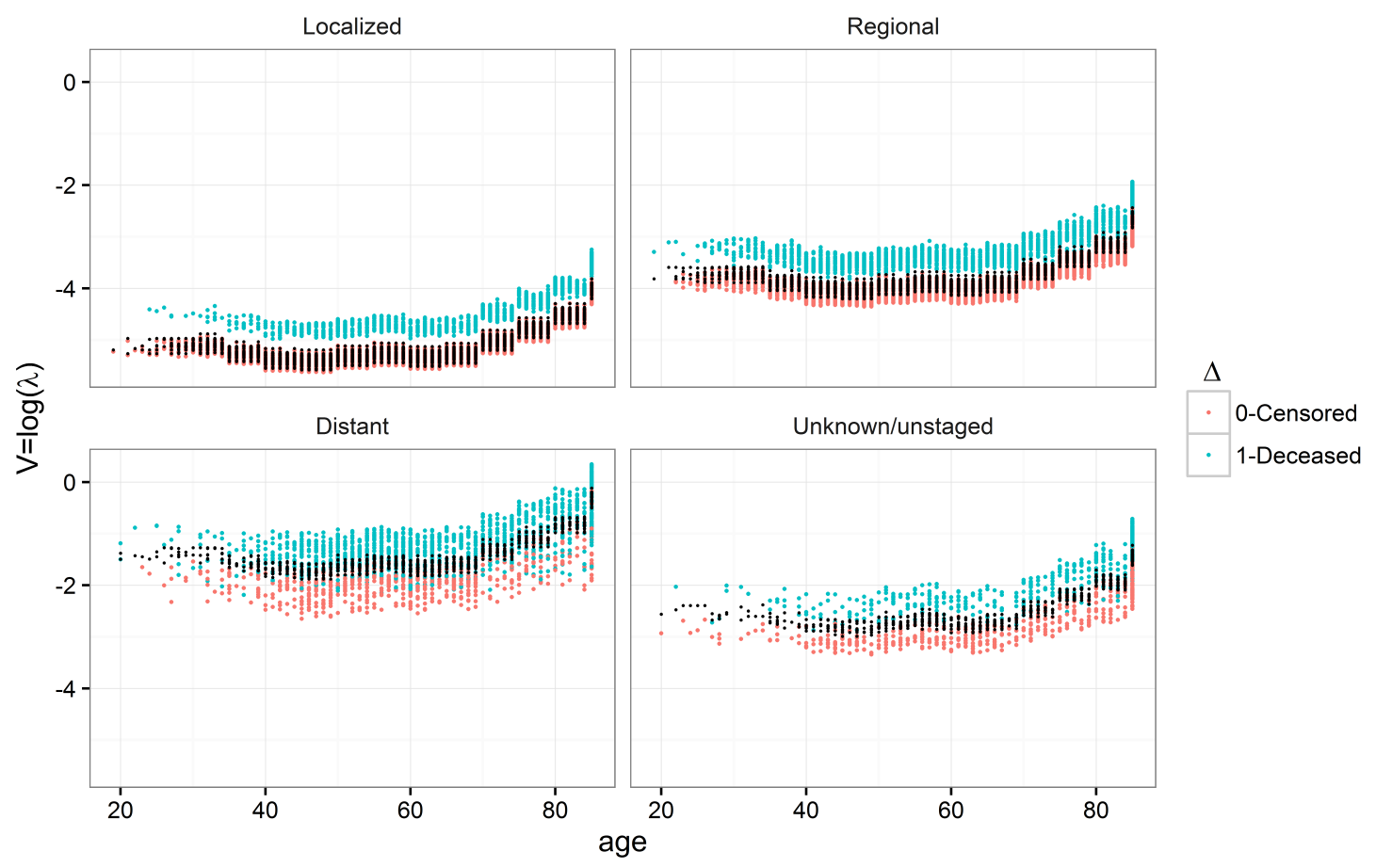

Figure 4.8: Scatter Plots of $v_{i}=\log \left(\lambda_{i}\right)$. The cyan points stand for the patients who died of breast cancer within our study period, the orange points stand for the right censored cases, and the black points are the means of $\boldsymbol{v}$, which are $\boldsymbol{X} \boldsymbol{\psi}$.

\section{Estimates of $v_{i}=\log \left(\lambda_{i}\right)$}

We also obtained the posterior means of $v_{i}=\log \left(\lambda_{i}\right)$. Figure 4.8 shows the scatter plots of $v_{i}$ s against patients' ages for different cancer stages. The plots we reach the same conclusion that the survival is worse for advanced stages. In addition, the non-linear age effects shows clear "U" shape patterns across all age groups. Mixing all the patients within counties, we plotted the average $v_{i}$ for each county in Figure 4.9. First we notice that the spatial association is not obvious given the map is not very smooth. Secondly, the overall pattern is similar to the late stage percentage distribution in Figure 4.4c. 


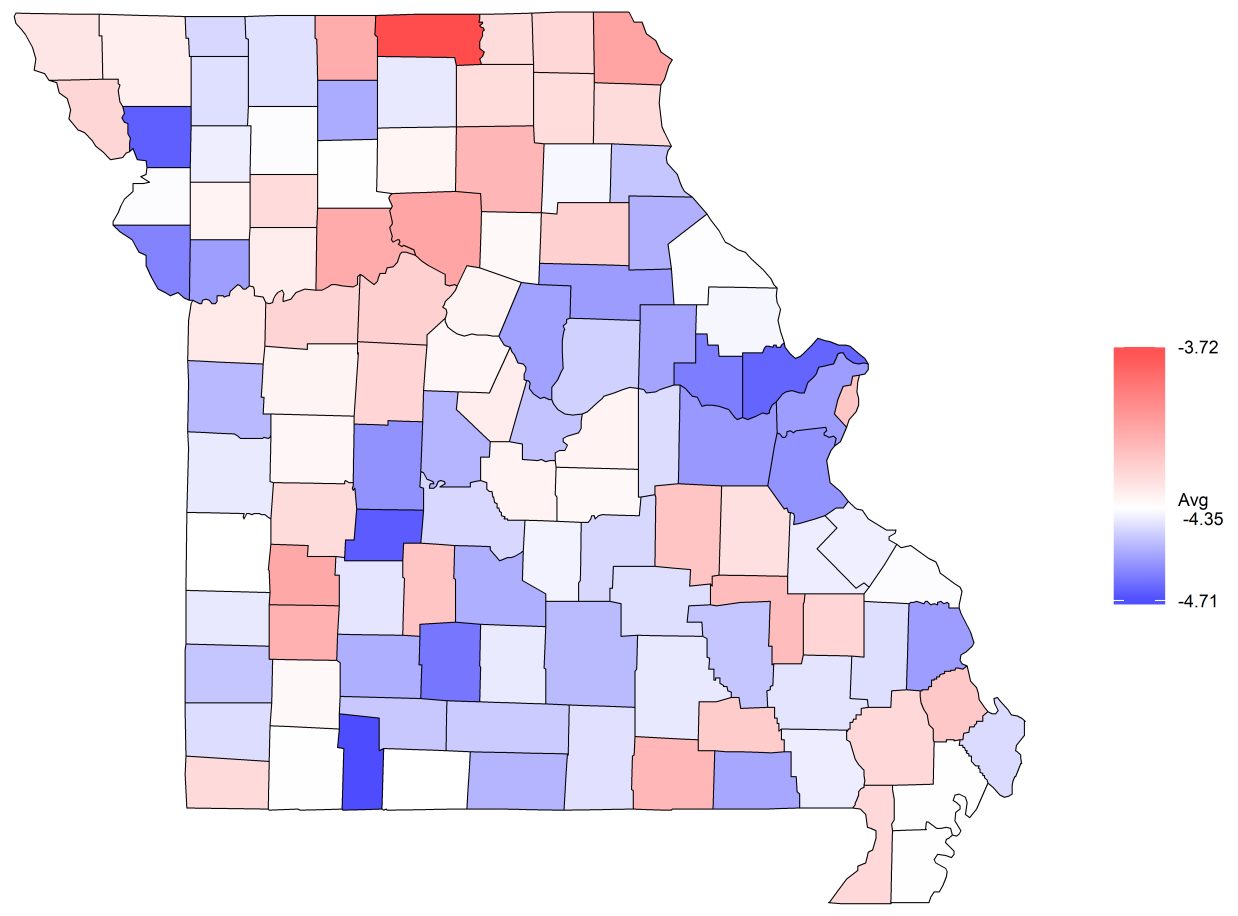

Figure 4.9: Map of Average $v_{i}=\log \left(\lambda_{i}\right)$ for Each County with All Stages and Ages Combined. White represents the state average. 


\section{Estimates of survival function}

Recall that (4.2) is the survival function based on parameter $\alpha$ and $\lambda_{i}$. We used posterior mean of $\alpha$ as its estimate. For the parameter $\lambda_{i}$, we wanted to examine the average value across all counties in Missouri. To achieve that, $\lambda_{i}$ is estimated as the posterior mean of the state average $\lambda_{i}$ calculated from posterior samples of $v_{i}$. For illustration purpose, we plotted the survival curve for women aged 34 or less, between 60 and 64 and 85 or above for all four different stages in Figure 4.10. Generally, advanced staged patients had worse survival. In addition, patients aged $i=34$ and $85+$ had worse survival than patients aged $60-64$.

\subsection{Discussion}

In this chapter, we extended the model in Dai et al. (2007) by assuming non-linear age effects and adding stage at diagnosis into our model. The model fitting results confirmed our non-linear modification and the importance of stage information. The breast cancer screening guideline for women in different age groups can be one of the reasons for the non-linear age effect. The American Cancer Society (ACS) recommends that average-risk women should undergo annual screening mammography beginning at 45 years of age; at age 55 women may transition to biennial screening, or continue with annual screening. Additionally, women 40 to 44 years should have the choice to begin annual screening. Women should continue screening as long as their overall health is good and they have a life expectancy of 10 years or more (Atlanta: American Cancer Society, Inc. 2015, 2014). Note that there are also different rec-

ommendations for breast cancer screening. The U.S. Preventive Services Task Force 


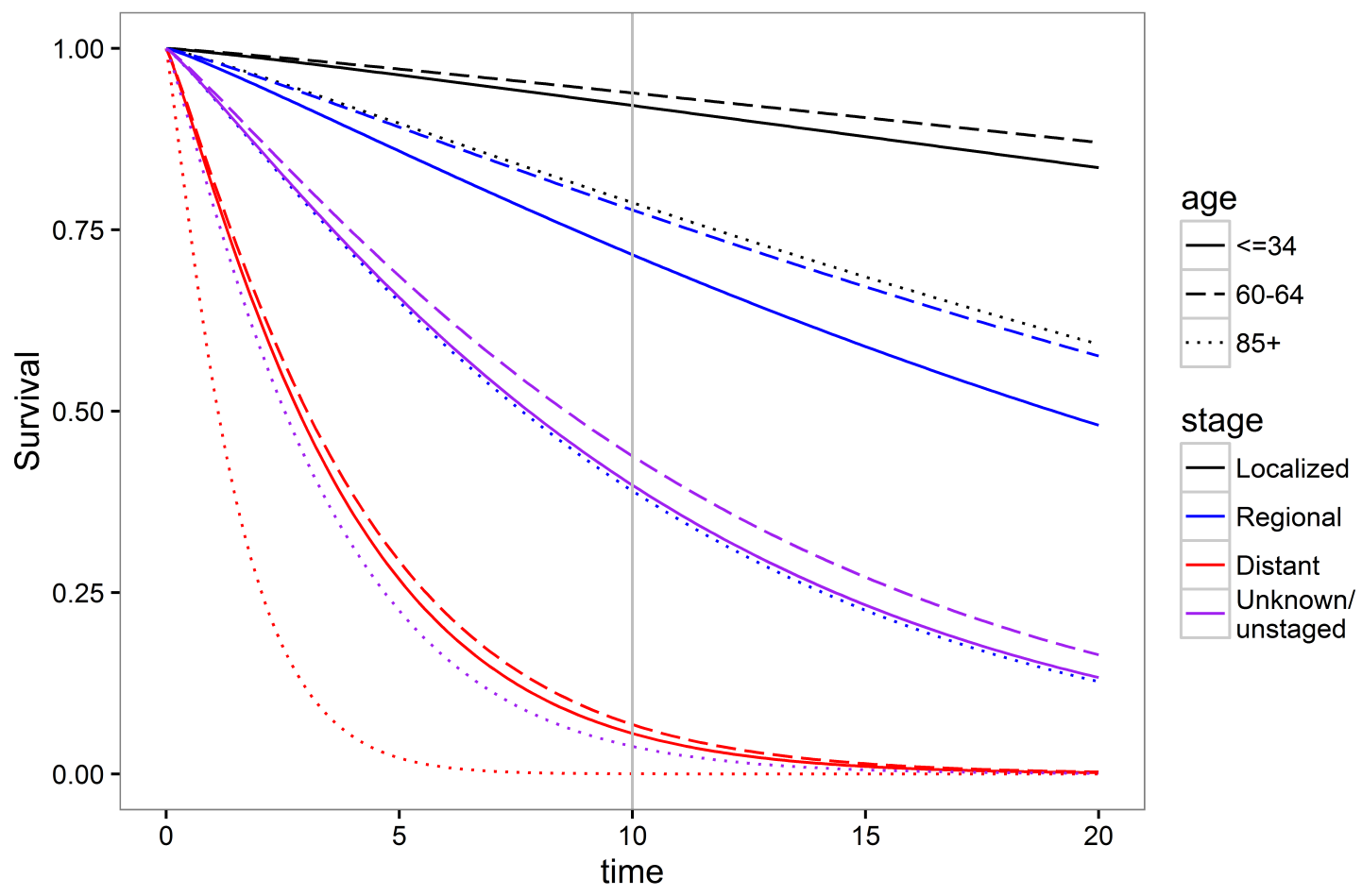

Figure 4.10: Estimated Survival Functions for Patients Aged $\leq 34,60-64$, and 85+ in Years. Parameter $\lambda_{i}$ in the survival function is based on posterior means of average $\lambda_{i} \mathrm{~s}$ across all the counties. Parameter $\alpha$ is the posterior mean of $\alpha$. Ten-year survival is fitted based on the observed data from 2001 to 2010. Note that the survival functions are being extrapolated out to 20 years. 
(USPSTF) recommends biennial screening mammography for women aged 50 to 74 years old, while the decision to start screening mammography in women prior to age 50 years should be an individual one. It also concludes that the current evidence is insufficient to assess the benefits and harms of screening mammography in women aged 75 years or older. Although the specific age to start/end breast cancer screening differs, ACS and USPSTF reach the same conclusion that younger and older women would not be expected to undergo regular screening. The lack of programmatic screening can be the reason for more advanced stages diagnosed for young women. Also, the regular screening for mid-aged women explains the increasing percentage of localized disease while the reduced screening practice for women aged 85 and over explains the percentage drop.

We also used random county effects as the spatial effects, which fitted the data well. The small variance of the spatial effects indicated weak spatial effect when estimating breast cancer survival in Missouri, which is a good phenomenon. For example, it may imply relatively uniform medical resources across Missouri. A more complicated model can be built by considering different spatial effects for different stages with interaction among them. However, it is still possible to only detect weak spatial effect. The large variance of the unstructured error term suggests that some other important variables are missing during our analysis of breast cancer survival, for example, it may help if we include race, tumor grade and tumor size information. Ideally, individual-level socioeconomic data such as education, income, etc. would be included. Unfortunately these data items are not in the Missouri Cancer Registry database. But an alternative potential future direction is by incorporating ecological analysis. 


\section{Chapter 5}

\section{A Scaled Spatio-temporal Model for Breast Cancer Treatment Delay}

Breast cancer is the most commonly diagnosed type of cancer and the second leading cause of cancer death among women in the United States. We call the waiting time between date of diagnosis and date of first treatment as treatment delay. The association between breast cancer survival and treatment delay has been established, but the disparities of treatment delay across different demographics, regions and years have not been studied much. In this chapter, we used the breast cancer treatment data to investigate the relationship between treatment delay and several covariates including race, cancer stage, age, county and year at diagnosis. A Hurdle Poisson likelihood was used for our Bayesian hierarchical models. The random effects were standardized by scaling their precision matrices. By scaling, the choice of hyper priors became more intuitive and the penalised-complexity (PC) priors were used in our study. Integrated nested laplace approximations (INLA) was used for Bayesian inference. 


\section{$5.1 \quad$ Introduction}

In our study, we analyzed the breast cancer treatment data obtained from Missouri Cancer Registry and Research Center. We define the treatment delay as the number of days between the date of diagnosis and the date of first treatment. The first treatment can be surgery, radiation, hormone therapy or biological response modifiers (BRM) therapy, whichever comes first. The data include all female breast cancer cases diagnosed from 1997 to 2014 in Missouri. We focused on investigating the relationship between treatment delay and several covariates. The covariates include patients' race, age at diagnosis, stage at diagnosis, year at diagnosis and county at diagnosis. Since the treatment delay is measured in days as non-negative integers, a Poisson regression framework can be a potential fit to our problem. However, we noticed that there are $27.37 \%$ of zeros for the treatment delay, which means some patients got same-day treatment. With the large amount of zeros, the Poisson distribution is not suitable anymore. The Zero-inflated Poisson (ZIP) and Hurdle Poisson (HP) models are often used to deal with excessive zeros. With a ZIP model, the response variable is modeled as a mixture of a Bernoulli distribution and a Poisson distribution. The zeros may come from either the Bernoulli process or the Poisson process. On the other hand, with a HP model, the Bernoulli process governs the binary outcome whether a count variate is zero or not. If the count is positive, the hurdle is crossed and the conditional distribution of the positives is governed by a truncated-at-zero Poisson distribution. Thus a HP model assumes the zeros all come from the Bernoulli process. In our case, our response variable is the delayed days. There can be multiple reasons for a patient to be treated on the same day or not. Once the patients didn't/couldn't receive the treatment on the day of diagnosis, their treatment would be delayed and our 
response variables would be positive. Therefore, an HP model fits better in our case. It enables us not only to model the delayed days through a Poisson regression, but also to model the probability of getting same-day treatment (zero-delay) through a logistic regression. We are interested in studying the changes of zero-delay probability and positive-delay days across patients' age, race, stage, year of diagnosis (time) and county at diagnosis (space).

A Bayesian hierarchical model was built for our treatment delay data with the HP distribution being on the top level. With the presence of spatial (county) and temporal (year) covariates, a spatio-temporal model was included in our framework. Spatiotemporal models have been widely used in the disease mapping literature. There are many choices to model the spatial effect, including the Besag model (Besag et al., 1991), the Leroux model (Leroux et al., 2000), the Dean model (Dean et al., 2001), etc. Those models assumes completely structured spatial effect. The BYM model (Besag et al., 1991) includes an unstructured spatial effect to make the model more flexible. Recently, Riebler et al. (2016) improved the BYM model by scaling the spatial effect based on the underlying graph structure, which is called BYM2 model. By scaling, the hyper parameters in the BYM2 model are easier to interpret and the choice of hyper priors are more sensible. For the temporal effect, the easiest way is to assume a linear model. If we want to model the nonlinearity, a second order random walk (RW2) (Rue and Held, 2005) model can be used. A RW2 model has the same structure as a second order smoothing spline (Kimeldorf and Wahba, 1970). One may also want to model the interaction effect between space and time. Knorr-Held (2000) proposed four types of spatio-temporal interactions: the Type I (unstructured spatial with unstructured temporal interaction), the Type II (structured spatial with unstructured temporal 
interaction), the Type III (unstructured spatial with structured temporal interaction) and the Type IV (structured spatial with structured temporal interaction). In our application, we adapted the idea of scaling from BYM2 model to scale all random effects in our model. The scaled random effects all have their generalized variance (Riebler et al., 2016) eqaul to one and then scaled by their corresponding precision parameters. The precision parameters are thus comparable and the choice of hyper priors are easier.

To get the parameter estimates in our Bayesian hierarchical model, we used integrated nested laplace approximations (INLA) for approximate Bayesian inference (Rue et al., 2009). This method is promising since it reduces the Bayesian computation substantially, especially like in our case with a large data set. Additionally, a practical advantage of INLA is that it can be used within $\mathbf{R}$ (Team et al., 2013) via the library R-INLA (Martino and Rue, 2009). Many latent Gaussian models have been implemented in R-INLA. With the use of INLA, we can get our Bayesian estimates without running long and complex MCMC algorithms.

In the remaining part of this chapter, we first introduce a scaled spatio-temporal model structure in Section 5.2. Then a Bayesian hierarchical model is built in Section 5.3 and the results are analyzed in Section 5.4. In the end, we conclude our paper in Section 5.6.

\subsection{A scaled spatio-temporal model}

Suppose $\eta_{k t}$ is the linear predictor from either the Logistic or the Poisson regression for area $k$ and time $t, \boldsymbol{\xi}=\left(\xi_{1}, \ldots, \xi_{K}\right)^{\prime}$ is the spatial effects for $K$ areas, $\gamma=\left(\gamma_{1}, \ldots, \gamma_{T}\right)^{\prime}$ 
is the temporal effects for $T$ time periods and $\boldsymbol{\delta}=\left(\delta_{11}, \ldots, \delta_{K 1}, \ldots, \delta_{1 T}, \ldots, \delta_{K T}\right)^{\prime}$ is the spatio-temporal interaction effects. We assumes the following linear model:

$$
\eta_{k t}=\alpha+\xi_{k}+\gamma_{t}+\delta_{k t}+\epsilon_{k t}
$$

where $\alpha$ is the intercept, $\xi_{k}$ is the spatial effect for area $k, \gamma_{t}$ is the the temporal effect for time $t, \delta_{k t}$ is the spatio-temporal interaction effect and $\epsilon_{k t}$ is the random error. In this section, we will review the BYM2 model used for our spatial effect, the RW2 model used for the temporal effect and the Type IV interaction effect.

\subsubsection{The BYM2 model}

The spatial effect $\boldsymbol{\xi}$ is defined on each area. For $K$ areas, recall that $\boldsymbol{\xi}=\left(\xi_{1}, \ldots, \xi_{K}\right)^{\prime}$. The spatial effect of area $k$ is $\xi_{k}$. It is reasonable to assume that the spatial effects are similar for areas close in space. Various spatial models has been developed to model such correlations. One of the most popular approaches is the Besag model (Besag et al., 1991). We use $k$ and $j$ stands for two areas. If they share common borders, we treat them as neighbors and denote it as $k \sim j$. For a county $k$, the collection of its neighbors is $d_{k}$ with size $n_{d_{k}}$. The Besag model assumes that the conditional distribution for the spatial effect $\xi_{k}$ is

$$
\xi_{k} \mid \boldsymbol{\xi}_{-k} \sim \mathrm{N}\left(\frac{1}{n_{d_{k}}} \sum_{j \in d_{k}} \xi_{j}, \frac{1}{n_{d_{k}} \tau_{\xi}}\right)
$$

where $\tau_{\xi}$ is the precision parameter and $\boldsymbol{\xi}_{-k}=\left(\xi_{1}, \ldots, \xi_{k-1}, \xi_{k+1}, \ldots, \xi_{K}\right)^{\prime}$. Thus the conditional mean of the area effect $\xi_{k}$ is the mean of the effects over its neighbors and 
the precision is proportional to the number of neighbors. The joint distribution for $\boldsymbol{\xi}$ is

$$
\boldsymbol{\xi} \propto \exp \left(-\frac{\tau_{\xi}}{2} \boldsymbol{\xi}^{\prime} \boldsymbol{Q}_{\xi} \boldsymbol{\xi}\right)
$$

where $\boldsymbol{Q}_{\xi}$ is the precision matrix with entries

$$
Q_{\xi, k j}= \begin{cases}n_{d_{k}} & k=j \\ -1 & k \sim j \\ 0 & \text { otherwise }\end{cases}
$$

Note that the rank of $\boldsymbol{Q}_{\xi}$ is $K-1$.

The limitation of the Besag model is that it only assumes spatially structured component which may give misleading parameter estimates when the true spatial component are just unstructured random errors. The BYM model (Besag et al., 1991) is often used to address such issue by decomposing the spatial effect into the unstructured and structured components, so that

$$
\xi=v+u
$$

Here $\boldsymbol{v} \sim \mathrm{N}_{K}\left(\mathbf{0}, \tau_{v}^{-1} \boldsymbol{I}\right)$ is the pure random noise and $\boldsymbol{u} \sim \mathrm{N}_{K}\left(\mathbf{0}, \tau_{u}^{-1} \boldsymbol{Q}_{\xi}^{-}\right)$is the Besag model (5.3), where $\boldsymbol{Q}_{\xi}^{-}$is the generalized inverse of $\boldsymbol{Q}_{\xi}$. Here we use $\mathrm{N}_{K}(\cdot, \cdot)$ to represent $K$ dimensional multivariate normal distribution and $\boldsymbol{I}$ is the identity matrix.

Riebler et al. (2016) introduced a modified BYM model (BYM2) by reparameterizing and scaling the BYM model. In the BYM2 model, the spatial random effect is 
modeled as

$$
\boldsymbol{\xi}=\frac{1}{\sqrt{\tau_{\xi}}}\left(\sqrt{1-\phi} \boldsymbol{v}+\sqrt{\phi} \boldsymbol{u}_{\star}\right)
$$

where $\tau_{\xi}$ is the overall precision parameter and $\phi \in[0,1]$ is the mixing parameter which distributes the variances between the unstructured random noise $\boldsymbol{v}$ and structured spatial component $\boldsymbol{u}_{\star}$. Here $\boldsymbol{v}$ follows the standard multivariate normal distribution $\mathrm{N}_{K}\left(\mathbf{0}, \boldsymbol{I}_{K}\right)$ and $\boldsymbol{u}_{\star}$ is the standardized spatial component after scaling the its precision matrix $\boldsymbol{Q}_{\xi}$, distributed as

$$
\boldsymbol{u}_{\star} \sim \mathrm{N}_{K}\left(\mathbf{0}, \boldsymbol{Q}_{\xi \star}^{-}\right)
$$

where $\boldsymbol{Q}_{\xi \star}$ is the scaled precision matrix such that the generalized variance of $\boldsymbol{u}_{\star}$ is 1. Note that there are no precision parameters in the distribution of $\boldsymbol{v}$ and $\boldsymbol{u}_{\star}$. For an unscaled random variable $\boldsymbol{u} \sim \mathrm{N}_{K}\left(\mathbf{0}, \boldsymbol{Q}_{\xi}^{-}\right)$, the marginal variances $\left[\boldsymbol{Q}_{\xi}^{-}\right]_{k k}$ depend on the graph structure $\boldsymbol{Q}_{\xi}$. Riebler et al. (2016) defines the generalized variance as the geometric mean of the marginal variances:

$$
\sigma_{G V}^{2}(\boldsymbol{u})=\frac{1}{K} \sum_{k=1}^{K} \log \left(\left[\boldsymbol{Q}_{\xi}^{-}\right]_{k k}\right) .
$$

To standardize $\boldsymbol{u}$, we may calculate $\boldsymbol{Q}_{\xi \star}$ by

$$
\boldsymbol{Q}_{\xi \star}=\sigma_{G V}^{2}(\boldsymbol{u}) \boldsymbol{Q}_{\xi}
$$

This scaling process removes the effect of graph structure on the generalized variance such that the variation contribution of the spatial component can be determined by $\phi / \tau_{\xi}$. Compared to BYM model, the benefit of the BYM2 model is that the 
hyper parameters $\tau_{\xi}$ and $\phi$ are more interpretable, which makes their prior choices more sensible. Recall in BYM model, the precision parameters $\tau_{v}$ and $\tau_{u}$ are not comparable since $\tau_{u}$ is the conditional precision given the graph structure and $\tau_{v}$ is the marginal precision. These two sources of variation can be confounded. In the BYM2 model, the variance

$$
\operatorname{Var}\left(\boldsymbol{\xi} \mid \tau_{\xi}, \phi\right)=\frac{1}{\sqrt{\tau_{\xi}}}\left((1-\phi) \boldsymbol{I}+\phi \boldsymbol{Q}_{\xi \star}^{-}\right)
$$

has a clear and intuitive interpretation as $\phi$ controls the variance distribution of the total variation. More importantly, by using the standardized $\boldsymbol{Q}_{\xi \star}^{-}$, the marginal variances will be approximately equal to $(1-\phi) / \tau_{\xi}+\phi / \tau_{\xi}$. In this sense, $\tau_{\xi}$ can be interpreted as the marginal precision for $\boldsymbol{\xi}$. It is easier to assign priors on $\tau_{\xi}$ when we are thinking about marginal precision or variance rather than the conditional version. In addition, when we have multiple random effects, standardizing them by scaling will enable us to compare their precision estimates to evaluate their importance.

\subsubsection{A scaled RW2 model}

A second order random walk (RW2) (Rue and Held, 2005) model can be used to model nonlinear relationship. For a length $n$ random vector $\boldsymbol{x}=\left(x_{1}, \ldots, x_{n}\right)^{\prime}$, a RW2 model defines the joint density of $\boldsymbol{x}$ :

$$
\begin{aligned}
\pi(\boldsymbol{x} \mid \kappa, \boldsymbol{Q}) & \propto \tau^{\left(n_{a}-2\right) / 2} \exp \left(-\frac{\tau}{2} \sum_{i=1}^{n-2}\left(x_{i}-2 x_{i+1}+x_{i+2}\right)^{2}\right) \\
& =\tau^{\left(n_{a}-2\right) / 2} \exp \left(-\frac{\tau}{2} \boldsymbol{x}^{\prime} \boldsymbol{Q} \boldsymbol{x}\right)
\end{aligned}
$$


where $\tau$ is the precision parameter and $\boldsymbol{Q}$ is the precision matrix with structure

$$
\boldsymbol{Q}=\left(\begin{array}{ccccccccc}
1 & -2 & 1 & & & & & & \\
-2 & 5 & -4 & 1 & & & & & \\
1 & -4 & 6 & -4 & 1 & & & & \\
& 1 & -4 & 6 & -4 & 1 & & & \\
& & \ddots & \ddots & \ddots & \ddots & \ddots & & \\
& & & 1 & -4 & 6 & -4 & 1 & \\
& & & & 1 & -4 & 6 & -4 & 1 \\
& & & & & 1 & -4 & 5 & -2 \\
& & & & & & 1 & -2 & 1
\end{array}\right)_{n \times n}
$$

Scaling can also be applied to the RW2 model. As in the BYM2 model, we first calculate the generalized variance and then scale the precision matrix.

For the temporal effect $\gamma=\left(\gamma_{1}, \ldots, \gamma_{T}\right)^{\prime}$, where $T$ is the number of time periods, we used the scaled RW2 model as:

$$
\boldsymbol{\gamma} \sim \mathrm{N}_{T}\left(\mathbf{0}, \tau_{\gamma}^{-1} \boldsymbol{Q}_{\gamma \star}^{-}\right)
$$

where $\tau_{\gamma}$ is the precision parameter and $\boldsymbol{Q}_{\gamma \star}$ is the scaled precision matrix based on $\boldsymbol{Q}_{\gamma}$, which has the same structure in (5.13) with dimension $T$.

\subsubsection{A scaled spatio-temporal interaction term}

With the format of $\boldsymbol{\xi}=\left(\xi_{1}, \ldots, \xi_{K}\right)^{\prime}$ and $\boldsymbol{\gamma}=\left(\gamma_{1}, \ldots, \gamma_{T}\right)^{\prime}$, the spatio-temporal interaction $\boldsymbol{\delta}$ is defined as $\boldsymbol{\delta}=\left(\delta_{11}, \ldots, \delta_{K 1}, \ldots, \delta_{1 T}, \ldots, \delta_{K T}\right)^{\prime}$. Assume a multivariate normal 
model on $\boldsymbol{\delta}$ with precision matrix $\boldsymbol{Q}_{\delta}$, Knorr-Held (2000) proposed four types of interactions. The Type I interaction assumes $\boldsymbol{Q}_{\delta}=\boldsymbol{I}_{T} \otimes \boldsymbol{I}_{K}$, Type II interaction assumes $\boldsymbol{Q}_{\delta}=\boldsymbol{I}_{T} \otimes \boldsymbol{Q}_{\xi}$, Type III interaction assumes $\boldsymbol{Q}_{\delta}=\boldsymbol{Q}_{\gamma} \otimes \boldsymbol{I}_{K}$ and Type IV interaction assumes $\boldsymbol{Q}_{\delta}=\boldsymbol{Q}_{\gamma} \otimes \boldsymbol{Q}_{\xi}$. The choice of the type of interaction term can be based on model selection (Ugarte et al., 2014). In our application, we only considered the Type IV interaction due to limited computation resources. In the end, we scale the precision matrix $\boldsymbol{Q}_{\delta}$ by its generalized variance to obtain the scaled $\boldsymbol{Q}_{\delta \star}$. Thus the prior for $\boldsymbol{\delta}$ is

$$
\boldsymbol{\delta} \sim \mathrm{N}_{K T}\left(\mathbf{0}, \tau_{\delta}^{-1} \boldsymbol{Q}_{\delta \star}^{-}\right),
$$

where $\tau_{\delta}$ is the precision parameter.

\subsection{Modeling the treatment delay}

\subsubsection{Data overview}

We obtained the breast cancer treatment data from Missouri Cancer Registry and Research Center. In our analysis, we only include female patients diagnosed from 1997 to 2014 in Missouri, with known race, stage, date at diagnosis, date of first treatment, age at diagnosis, county at diagnosis. In addition, we only consider cases where breast was the primary site or first one if multiple tumors detected. We calculated the delayed days as the difference between date of first treatment and date at diagnosis. There were less than $0.11 \%$ of cases with delayed days over 365 (a year). We treated them as outliers and removed them. In addition, since most of the patients are white $(66,347)$ and black $(7204)$, we decided to remove patients from all the other races 


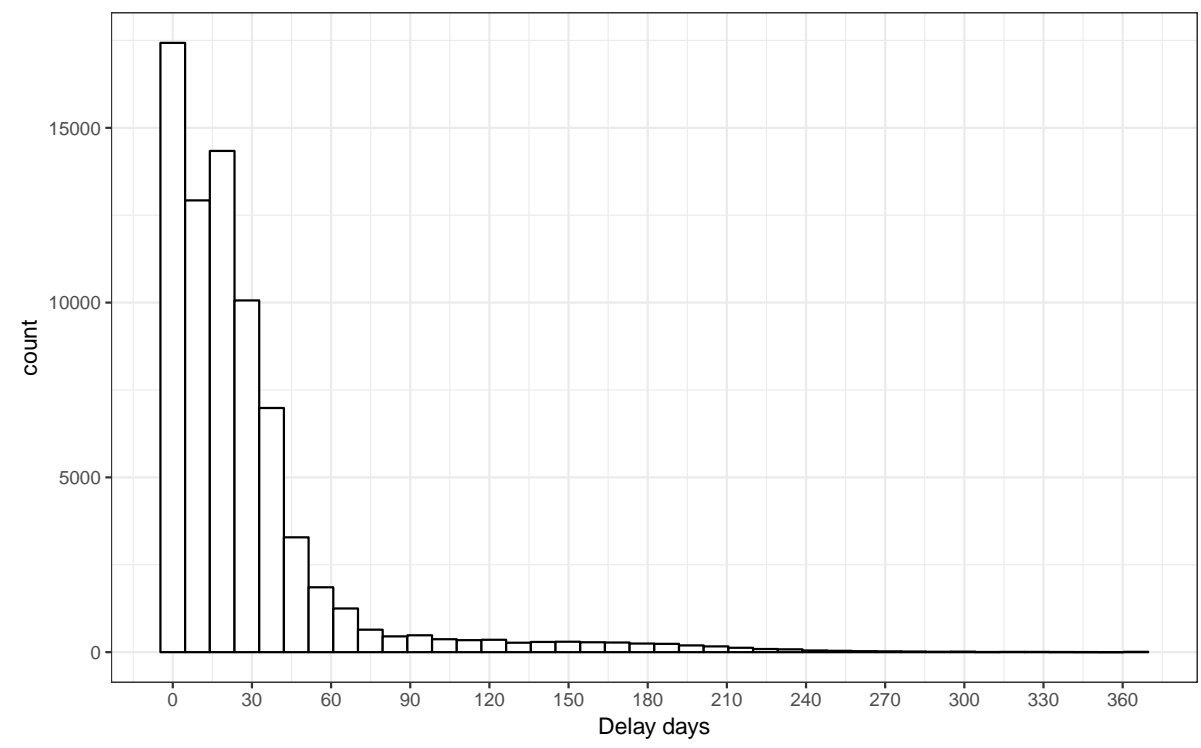

Figure 5.1: Histogram of Treatment Delay Measured in Days.

(959). In the end, there are 73,551 observations in our study. We noticed that there are $15,824(27.41 \%)$ cases has zero delayed days. The overall distribution for the delayed days are shown in Figure 5.1. The distribution is skewed to the left with a peak at zero. To visualize the delayed days for counties in Missouri over the years, we plotted the proportion of zero delays and the average positive delayed days in Figure 5.2. We aggregated data for each county over every two years time period. For each county in a time period, the proportion of zero delays was calculated as the proportion of zeros. The average positive delayed days was calculated as the mean of the delayed days after removing all zeros. From the spatio-temporal plot, we can see there may exist patterns in space and time for our treatment delay. 
(a) Proportion of Zero Delay
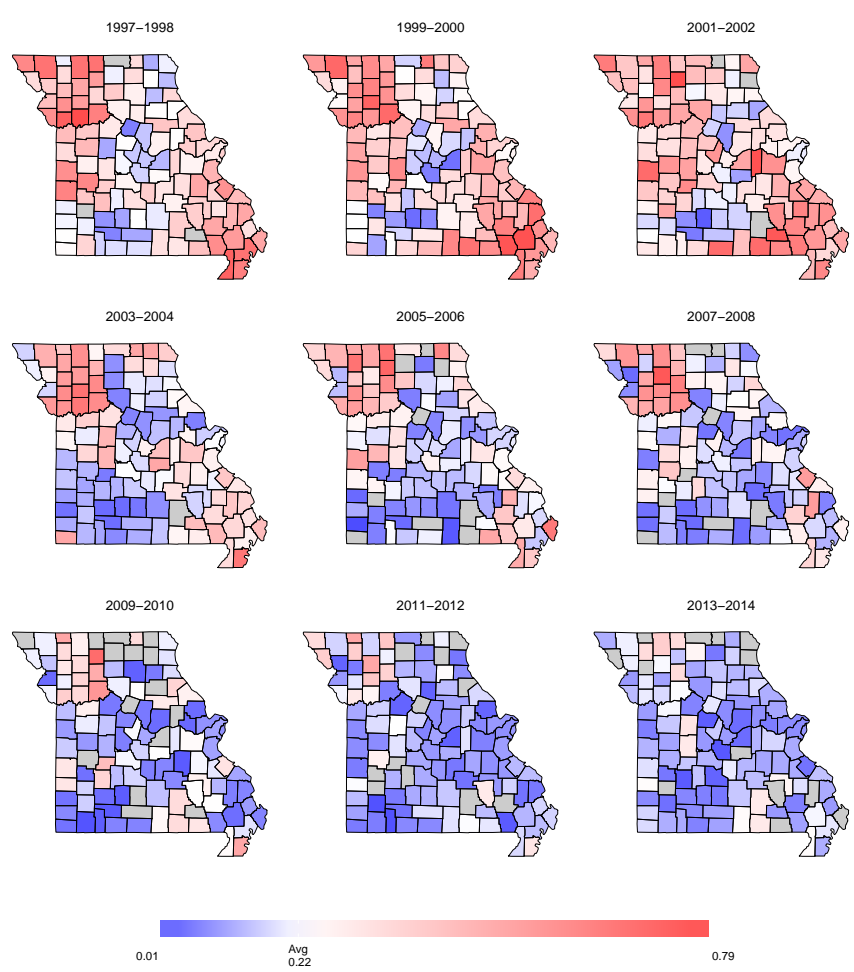

(b) Average Delayed Days
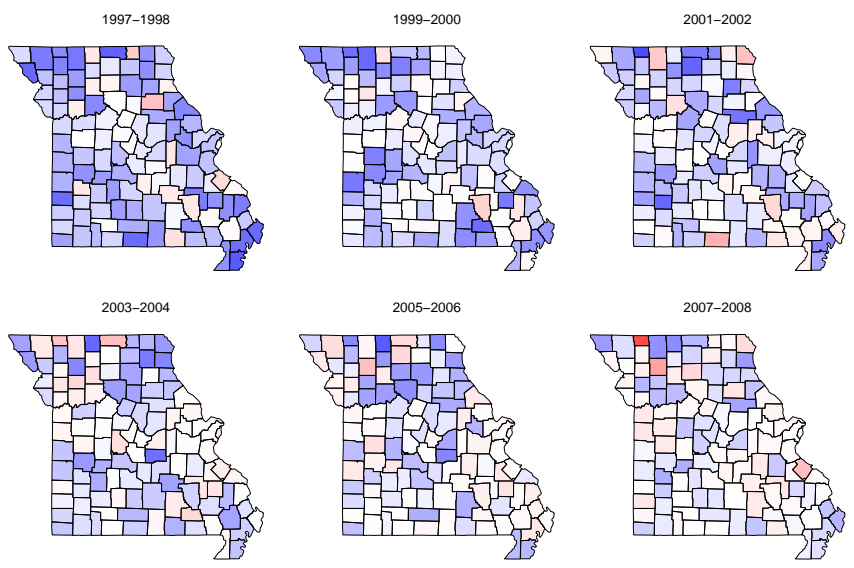

2009-2010
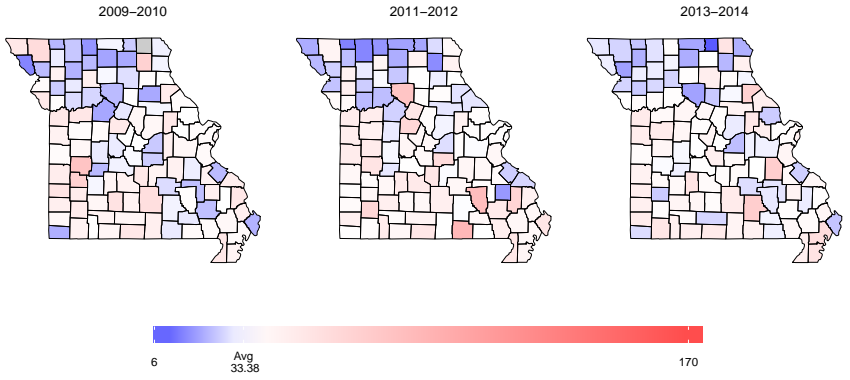

Figure 5.2: The Proportion of Zero Delay and the Average Positive Delayed Days for Each County in Missouri Over Years. The white color stands for the statewide average over all years. The gray color stands for missing data. 


\subsubsection{Likelihoods and linear models}

We are interested in studying the changes of treatment delay across patients' race, stage, age, county (space) and year (time) of diagnosis. For the race variable, we divided the patients into the white group and non-white group. For the stage variable, we have In Situ, Localized, Regional, Distant and Unknown stages. For the age variable, we used 8 age groups as ¡30, 30-39, 40-49, 50-59, 60-69, 70-79, 80-89 and $90+$. For the county variable, we labeled the 114 counties and City of St. Louis from 1 to 115. For the year variable, we group every two years together from 1997 to 2014, which gave us 12 time periods in total. As for the notation, let $y_{i}$ be the delayed days for patient $i$, which takes value 0 or a positive integer. For this patient $i$, the covariates are coded as:

- $r_{i}$ : race, equals either 1 (white) or 2 (black);

- $s_{i}$ : stage at diagnosis, which takes value among 1 (in Situ), 2 (Localized), 3 (Regional), 4 (Distant) and 5 (Unknown);

- $a_{i}$ : age at diagnosis, $a_{i} \in\left(1, \ldots, n_{a}=8\right)$;

- $c_{i}$ : county at diagnosis, $c_{i} \in(1, \ldots, K=115)$;

- $t_{i}$ : year at diagnosis, $t_{i} \in(1, \ldots, T=12)$.

Assume $y_{i}$ follows a HP model, then the likelihood function of $y_{i}$ is

$$
\begin{aligned}
L\left(y_{i} \mid p_{i}, \lambda_{i}\right) & =p_{i} \times 1_{\left[y_{i}=0\right]}+\left(1-p_{i}\right) \times \operatorname{Poisson}\left(y_{i} \mid \lambda_{i}, y_{i}>0\right) \\
& =p_{i} \times 1_{\left[y_{i}=0\right]}+\left(1-p_{i}\right) \times \frac{\lambda_{i}^{y_{i}}}{\left(\exp \left(\lambda_{i}\right)-1\right) y_{i} !} \times 1_{\left[y_{i}>0\right]}
\end{aligned}
$$


To model the probability of same-day treatment and number of delayed days, we used

$$
\eta_{i}^{(0)}=\log \left(\frac{p_{i}}{1-p_{i}}\right)=\alpha^{(0)}+\beta_{r_{i}}^{(0)}+\omega_{s_{i}}^{(0)}+\theta_{a_{i}}^{(0)}+\xi_{c_{i}}^{(0)}+\gamma_{t_{i}}^{(0)}+\delta_{c_{i} t_{i}}^{(0)}+\epsilon_{i}^{(0)}
$$

and

$$
\eta_{i}^{(1)}=\log \left(\lambda_{i}\right)=\alpha^{(1)}+\beta_{r_{i}}^{(1)}+\omega_{s_{i}}^{(1)}+\theta_{a_{i}}^{(1)}+\xi_{c_{i}}^{(1)}+\gamma_{t_{i}}^{(1)}+\delta_{c_{i} t_{i}}^{(1)}+\epsilon_{i}^{(1)},
$$

where the superscript $m \in(0,1)$ stands for the parameters for the logistic regression (0) or the Poisson regression (1). Thus for each regression model,

- $\boldsymbol{\eta}^{(m)}=\left(\eta_{1}^{(m)}, \ldots, \eta_{N}^{(m)}\right)^{\prime}$ is the linear predictor either from logit or log transformation;

- $\alpha^{(m)}$ is the intercept;

- $\boldsymbol{\beta}^{(m)}=\left(\beta_{1}^{(m)}, \beta_{2}^{(m)}\right)^{\prime}$ is the race effect for whites and not-whites;

- $\boldsymbol{\omega}^{(m)}=\left(\omega_{1}^{(m)}, \ldots, \omega_{5}^{(m)}\right)^{\prime}$ is the stage effect;

- $\boldsymbol{\theta}^{(m)}=\left(\theta_{1}^{(m)}, \ldots, \theta_{n_{a}}^{(m)}\right)^{\prime}$ is age effect;

- $\boldsymbol{\xi}^{(m)}=\left(\xi_{1}^{(m)}, \ldots, \xi_{K}^{(m)}\right)^{\prime}$ is the spatial (county) effect;

- $\gamma^{(m)}=\left(\gamma_{1}^{(m)}, \ldots, \gamma_{T}^{(m)}\right)^{\prime}$ is the temporal (year at diagnosis) effect;

- $\boldsymbol{\delta}^{(m)}=\left(\delta_{11}^{(m)}, \ldots, \delta_{K 1}^{(m)}, \ldots, \delta_{1 T}^{(m)}, \ldots, \delta_{K T}^{(m)}\right)^{\prime}$ is spatio-temporal interaction effect;

- $\boldsymbol{\epsilon}^{(m)}=\left(\epsilon_{1}^{(m)}, \ldots, \epsilon_{N}^{(m)}\right)^{\prime}$ is the patient-specific error term. 


\subsubsection{Priors on regression parameters}

\section{Priors for the fixed effects}

We treated the intercept $\alpha^{(m)}$, race effect $\boldsymbol{\beta}^{(m)}$ and stage effect $\boldsymbol{\omega}^{(m)}$ as fixed effects. Normal distributions with relative large variances were used as their prior distributions. To be specific,

$$
\alpha^{(m)} \sim \mathrm{N}\left(0, \sigma_{f}^{2}\right), \quad \boldsymbol{\beta}^{(m)} \sim \mathrm{N}_{2}\left(\mathbf{0}, \sigma_{f}^{2} \boldsymbol{I}\right), \quad \text { and } \quad \boldsymbol{\omega}^{(m)} \sim \mathrm{N}_{5}\left(\mathbf{0}, \sigma_{f}^{2} \boldsymbol{I}\right),
$$

where $\mathbf{0}$ is vector of zeros and $\sigma_{f}^{2}$ is a large fixed number to specify the variances. We set $\sigma_{f}^{2}=e^{4}$ in our application.

\section{Priors for the spatial effects}

For the spatial random effect $\boldsymbol{\xi}^{(m)}$ defined on counties in Missouri, we used the BYM2 model discussed in Section 5.2.1. To be specific, let $\boldsymbol{Q}_{\xi}$ be the precision matrix defined in (5.4) based on the neighborhood structure of counties in Missouri. Follow the structure of BYM2 model, we model $\boldsymbol{\xi}^{(m)}$ as

$$
\boldsymbol{\xi}^{(m)}=\frac{1}{\sqrt{\tau_{\xi}^{(m)}}}\left(\sqrt{1-\phi^{(m)}} \boldsymbol{v}^{(m)}+\sqrt{\phi^{(m)}} \boldsymbol{u}_{\star}^{(m)}\right)
$$

where $\tau_{\xi}^{(m)}$ is the overall precision parameter and $\phi^{(m)} \in[0,1]$ is the mixing parameter. Again, $\boldsymbol{v}^{(m)} \sim \mathrm{N}_{K}(\mathbf{0}, \boldsymbol{I})$ is the unstructured random noise and $\boldsymbol{u}_{\star}^{(m)}$ is the standardized spatial component after scaling the its precision matrix $\boldsymbol{Q}_{\xi}$, distributed as

$$
\boldsymbol{u}_{\star}^{(m)} \sim \mathrm{N}_{K}\left(\mathbf{0}, \boldsymbol{Q}_{\xi \star}^{-}\right),
$$


where $\boldsymbol{Q}_{\xi \star}$ is the scaled precision matrix such that the generalized variance of $\boldsymbol{u}_{\star}^{(m)}$ is 1.

\section{Priors for the age and temporal effects}

Since the relationship between the linear predictor $\boldsymbol{\eta}^{(m)}$ and age effect $\boldsymbol{\theta}^{(m)}$ or temporal effect $\gamma^{(m)}$ may not be linear, we used scaled RW2 model as in Section 5.2.2. Let $\boldsymbol{Q}_{\theta}$ and $\boldsymbol{Q}_{\gamma}$ be the precision matrices of dimension $n_{a}$ and $T$ as in (5.13), then we obtained the scaled version as $\boldsymbol{Q}_{\theta \star}$ and $\boldsymbol{Q}_{\gamma \star}$. A sclaed RW2 prior assigns the folowing prior distributions on $\boldsymbol{\eta}^{(m)}$ and $\boldsymbol{\theta}^{(m)}$ :

$$
\boldsymbol{\theta}^{(m)} \sim \mathrm{N}_{n_{a}}\left(\mathbf{0}, \frac{1}{\tau_{\theta}^{(m)}} \boldsymbol{Q}_{\theta \star}^{-}\right) \quad \text { and } \quad \boldsymbol{\gamma}^{(m)} \sim \mathrm{N}_{T}\left(\mathbf{0}, \frac{1}{\tau_{\gamma}^{(m)}} \boldsymbol{Q}_{\gamma \star}^{-}\right)
$$

where $\tau_{\theta}^{(m)}$ and $\tau_{\gamma}^{(m)}$ are the corresponding precision parameters.

\section{Prior for the spatio-temporal interaction effects}

We used the Type IV interaction model in Section 5.2.3 directly. Let $\boldsymbol{Q}_{\delta}=\boldsymbol{Q}_{\gamma} \otimes \boldsymbol{Q}_{\xi}$ be the unscaled precision matrix for $\boldsymbol{\theta}^{(m)}$. By multiplying the generalized variance, we obtained the scaled precision matrix $\boldsymbol{Q}_{\delta \star}$. Therefore, the prior distribution for $\boldsymbol{\theta}^{(m)}$ is

$$
\boldsymbol{\delta}^{(m)} \sim \mathrm{N}_{K T}\left(\mathbf{0}, \frac{1}{\tau_{\delta}^{(m)}} \boldsymbol{Q}_{\delta \star}^{-}\right)
$$

where $\tau_{\delta}^{(m)}$ is the precision parameter for $\boldsymbol{\delta}^{(m)}$. 


\section{Prior for the error term}

For the patient-specific error $\boldsymbol{\epsilon}^{(m)}$, we treated them as random noise and had Ndimentional multivariate normal distribution

$$
\boldsymbol{\epsilon}^{(m)} \sim \mathrm{N}_{N}\left(\mathbf{0}, \frac{1}{\tau_{\epsilon}^{(m)}} \boldsymbol{I}\right)
$$

where $\tau_{\epsilon}^{(m)}$ is the precision. Note that for a standardized multivariate normal distribution, the generalized variance is 1 . Thus we don't need to scale the error term.

\subsubsection{Identifiability constraints}

The race effect $\boldsymbol{\beta}^{(m)}$ and the stage effect $\boldsymbol{\omega}^{(m)}$ are confounded with the intercept $\alpha^{(m)}$. Thus, we added sum-to-zero constraints on $\boldsymbol{\beta}^{(m)}$ and $\boldsymbol{\omega}^{(m)}$. Specifically, for each $m=1$ or 2 we need:

$$
\mathbf{1}_{2} \boldsymbol{\beta}^{(m)}=0 \quad \text { and } \quad \mathbf{1}_{5} \boldsymbol{\omega}^{(m)}=0 .
$$

In addition to the fixed effects above, the random effects in our model should also be fitted with certain constrains to eliminate confounding issues. Goicoa et al. (2017) discussed the necessary constrains needed for commonly used random effect models in spatio-temporal disease mapping literature. For the spatial effect $\boldsymbol{\xi}^{(m)}$, the constrains should actually be placed on its intrinsic component $\boldsymbol{u}_{\star}^{(m)}$ :

$$
\mathbf{1}_{K} \boldsymbol{u}_{\star}^{(m)}=0, \quad \text { for } \quad m=1,2 .
$$


For the RW2 models used on the age effect $\boldsymbol{\theta}^{(m)}$ and temporal effect $\boldsymbol{\gamma}^{(m)}$, the constrains are:

$$
\mathbf{1}_{n_{a}} \boldsymbol{\theta}^{(m)}=0, \quad \text { for } \quad m=1,2 .
$$

and

$$
\mathbf{1}_{T} \boldsymbol{\gamma}^{(m)}=0, \quad \text { for } \quad m=1,2
$$

For the spatio-temporal interaction effect $\boldsymbol{\delta}^{(m)}$, the constrains are:

$$
\mathbf{1}_{T} \boldsymbol{\delta}_{k \cdot}^{(m)}=0 \quad \text { and } \quad \mathbf{1}_{K} \boldsymbol{\delta}_{\cdot t}^{(m)}=0 \quad \text { for } \quad m=1,2 ; \quad k=1, \ldots, K ; \quad t=1, \ldots, T
$$

where $\boldsymbol{\delta}_{k, \cdot}^{(m)}=\left(\delta_{k 1}^{(m)}, \delta_{k 2}^{(m)}, \ldots, \delta_{k T}^{(m)}\right)^{\prime}$ and $\boldsymbol{\delta}_{\cdot t}^{(m)}=\left(\delta_{1 t}^{(m)}, \delta_{2 t}^{(m)}, \ldots, \delta_{K t}^{(m)}\right)^{\prime}$. Additionally, the constraints

$$
\sum_{k=1}^{K} \sum_{t=1}^{T} t \delta_{k t}^{(m)}=0
$$

should be satisfied, but they are automatically placed with the constrains in (5.30).

\subsubsection{Choice of hyperpriors: the penalised-complexity priors}

In the spatio-temporal disease mapping literature, priors on the precision parameters are usually specified rather ad-hoc (Bernardinelli et al., 1995). In most cases, Gamma distributions are used, but how to choose the shape and rate parameters for the Gamma distribution is not clear. In addition, the priors from one study may not be transfered to another one due to different graph structure. The scaling procedure used in our model removed the effects of the graph structure and standardized all random variables. More importantly, the precision parameters $\tau_{\epsilon}^{(m)}, \tau_{\xi}^{(m)}, \tau_{\theta}^{(m)}, \tau_{\gamma}^{(m)}$ and $\tau_{\delta}^{(m)}$ can be interpreted as marginal precision and are comparable with each other. 
Therefore, it is easier to assign priors if we have a rough sense about the magnitude of those precisions or variances. Aside from Gamma distribution on the precision parameter, there are other methods to assign priors. For example, one can place uniform, half-Cauchy or half-t distributions on the standard deviation parameter (Gelman et al., 2006) and then transform to the precision level.

Here, we will use the framework of penalised-complexity $(\mathrm{PC})$ priors proposed by Simpson et al. (2017). The PC priors were also used in Riebler et al. (2016) for the BYM2 model in Section 5.2.1. In general, the PC prior framework uses the KullbackLeibler distance (KLD) to measure the increased complexity when assigning certain priors. The simpler models are preferred until a more complex model is supported by the data. In addition, the PC prior assumes the deviation from the simpler model is penalized using a constant decay rate. The parameters in the PC priors can be chosen when people has an idea of a sensible size of the parameter or any transformed variation of interest. In the following part, we will present the PC priors used in our model. We omit the derivations of those priors. Simpson et al. (2017) contains the full details.

\section{On the precision parameters}

For the precision parameters, Simpson et al. (2017) suggested a type-2 Gumbel distribution as the PC prior. Let $\tau$ be a precision parameter, then the type-2 Gumbel distribution has the density

$$
[\tau \mid b]=\frac{b}{2} \tau^{-3 / 2} \exp \left(-b \tau^{-1 / 2}\right) .
$$


This prior corresponds to an exponential prior with decay rate $b$ for the standard deviation $1 / \sqrt{\tau}$. The value of $b$ can be inferred by the probability statement $\operatorname{Prob}((1 / \sqrt{\tau})>$ $U)=a$, which gives $b=-\log (a) / U$. As a rule of thumb, for a random variable $\boldsymbol{x} \sim N\left(\mathbf{0}, \tau^{-1} \boldsymbol{I}\right)$, the marginal standard deviation, after the type-2 Gumbel distribution for $\tau$ is integrated out, is about $0.31 U$ when $a=0.01$ (Simpson et al., 2017). Thus we can use this rule to select the parameter $U$ if we have a sense of how large the marginal standard deviation could be.

In our model, we have $\tau_{\epsilon}^{(m)}, \tau_{\xi}^{(m)}, \tau_{\theta}^{(m)}, \tau_{\gamma}^{(m)}$ and $\tau_{\delta}^{(m)}$ as our precision parameters. Since the corresponding random effects have been scaled, we can place the same priors for the same $m$. For $m=0$ which corresponding to the logistic part, the predicted probabilities from the regression model might mostly be in the range $[0.05,0.95]$ leading to an interval $[2.94,2.94]$ on the linear predictor scale. Thus we assume there is only a small chance for the standard deviation to exceed 2.94/1.96, where 1.96 is the upper $5 \%$ quantile for a standard normal distribution. Applying the rule of $0.31 U$, we have the parameters $\left(U_{0}=4.84, a=0.01\right)$ for the precisions $\tau_{\epsilon}^{(0)}, \tau_{\xi}^{(0)}, \tau_{\theta}^{(0)}$, $\tau_{\gamma}^{(0)}$ and $\tau_{\delta}^{(0)}$. For $m=1$ which corresponding to the Poisson regression, since the number of delayed days ranges from 1 to 365 , the range on the linear predictor scale is $[0, \log (365)]$. Thus we assume the standard deviation only has a very small chance of greater than $\log (365) / 2 / 1.96$. Together with the rule of $0.31 U$, we end up with the parameters $\left(U_{1}=4.86, a=0.01\right)$ for the precisions $\tau_{\epsilon}^{(1)}, \tau_{\xi}^{(1)}, \tau_{\theta}^{(1)}, \tau_{\gamma}^{(1)}$ and $\tau_{\delta}^{(1)}$.

To visualize the effect of $U$ and $a$ on the PC prior for the precision parameter, we plotted the densities for three set of parameters in Figure 5.3. Keeping a constant, reducing $U$ will make the density less skewed to zero. Keeping $U$ constant, increasing $a$ makes the density more skewed to zero. 


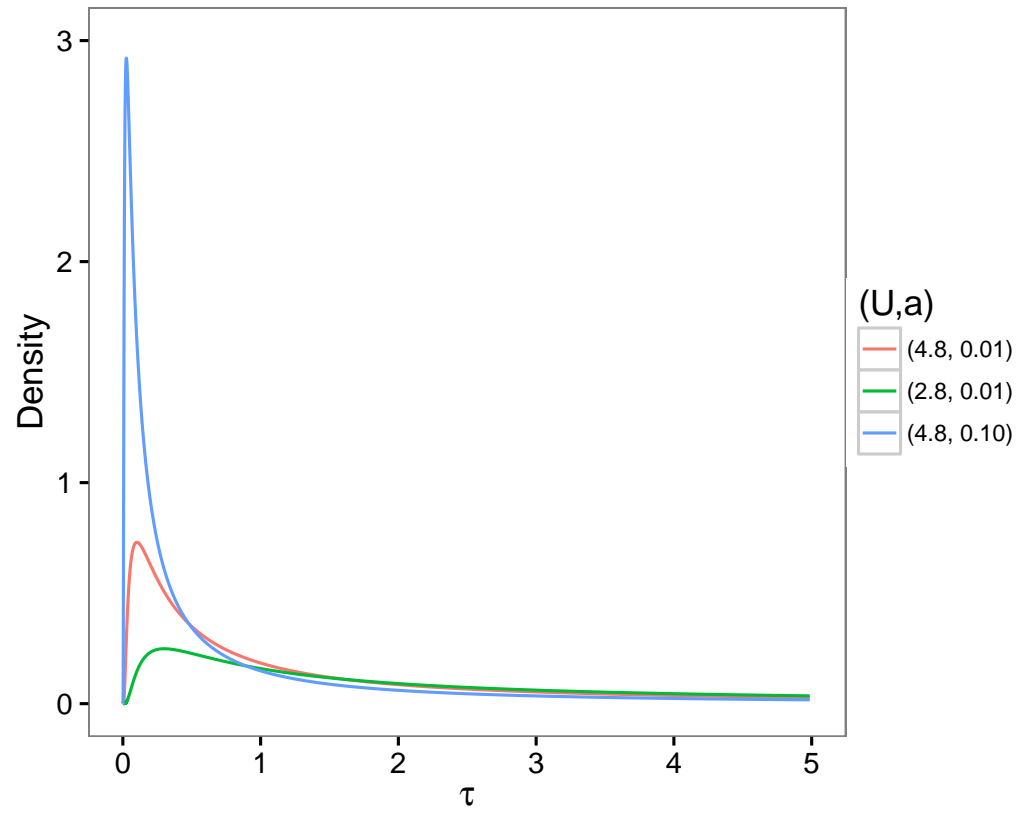

Figure 5.3: Density Plot for the PC Prior on Precision Parameter.

\section{On the mixing parameters}

Recall that in the BYM2 model (5.6), the mixing parameter $\phi^{(m)}$ measures how much variance can be accounted by the structured spatial component. The derivation of the PC prior on $\phi^{(m)}$ requires the computation of the KLD between the base model and the flexible model (Simpson et al., 2017). For the BYM2 model, the base model has covariance matrices $\boldsymbol{\Sigma}_{\text {base }}=\boldsymbol{I}$, while the flexible model has covariance matrix $\boldsymbol{\Sigma}_{f l e x}\left(\phi^{(m)}\right)=\left(1-\phi^{(m)}\right) \boldsymbol{I}+\phi^{(m)} \boldsymbol{Q}_{\xi \star}^{-}$. An exponential prior with parameter $h$ is assigned to the distance scale

$$
d\left(\phi^{(m)}\right)=\sqrt{2 \operatorname{KLD}\left[N\left(\mathbf{0}, \boldsymbol{\Sigma}_{\text {flex }}\left(\phi^{(m)}\right)\right) \mid N\left(\mathbf{0}, \boldsymbol{\Sigma}_{\text {base }}\right)\right]} .
$$




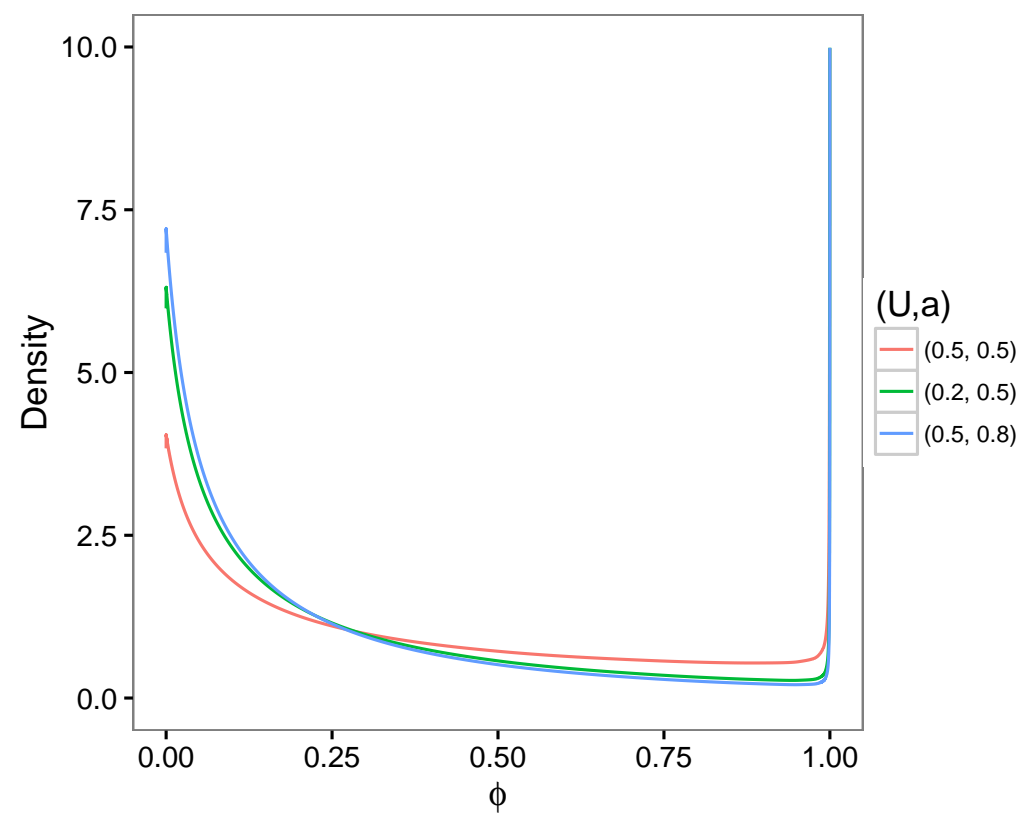

Figure 5.4: Density Plot for the PC Prior on Precision Parameter.

The value of the decay parameter $h$ can be obtained using the probability statement $\operatorname{Prob}\left(\left(\phi^{(m)}\right)<U\right)=a$. Different from the case for precision parameters, the interpretation for $\phi^{(m)}$ is more straightforward. A large value of $\phi^{(m)}$ puts more weights on the structured spatial effects. In our analysis, we used $\left(U_{\phi}=0.5, a_{\phi}=0.5\right)$ to specify the priors for $\phi^{(m)}, m=1$ and 2. Note that the resulting PC prior for $\phi^{(m)}$ has no closed form, which must be computed numerically. In addition, the choice of $U$ and $a$ for this mixing parameter can potentially have sensitive impact on the resulting posterior distributions (Riebler et al., 2016). Other than this PC prior, one can also use uniform distribution or beta distributions for the mixing parameters. Figure 5.4 shows the density plots for different values of $U$ and $a$. Generally, this PC prior put more weights on smaller values of $\phi^{(m)}$ towards to base model. The values of $U$ and a represent for different level of preference to the base model. 


\subsection{Result analysis}

\subsubsection{Computation}

Markov Chain Monte Carlo (MCMC) is often used in the context of Bayesian hierarchical modeling. In our application, there are 73,551 observations in total and 57,727 of them have positive delays. For a Hurdle Poisson model, we will have to estimate 131,278 linear predictors along with all other parameters in a single MCMC iteration. Building the MCMC algorithm is prolong and the computation time until convergence will be extreme long. To avoid such problem, we used the INLA package in $\mathrm{R}$ for approximate Bayesian inference. We ran the model on a computer cluster with 64 CPUs (Intel Xeon E5-2686 v4). The peak memory consumed was around 120 Gigabyte. In the end, the total running time is around 71 hours.

\subsubsection{Hyper parameter estimates}

Table 5.1 displays the summary statistics for the hyper parameters based on their posterior distribution. For our Hurdle Poisson regression, we separated our results into the Logistic and the Poisson parts. For each part, there are five precision parameters for the random error, age, temporal (year), spatial (county) and spatio-temporal interaction effects, along with the mixing parameter $\phi^{(m)}$ in the BYM2 model. Since all the random effects are standardized, their precision parameters are comparable. A smaller precision may indicate a major source of variation. For the Logistic part, the precision parameter for the spatial effect $\tau_{\xi}^{(0)}$ takes the smallest value, which means the spatial effect contributes most of the variation in our model. The large precision parameter for the error term in the Logistic part suggests we may have included 
Table 5.1: Summary Statistics for Posterior Distributions of Hyper Parameters

\begin{tabular}{lllrrrrrr}
\hline Model & Para. & Description & Mean & Sd & $2.5 \%$ & Median & $97.5 \%$ & Mode \\
\hline \multirow{5}{*}{ Logistic } & $\tau_{\epsilon}^{(0)}$ & error & 389.21 & 1263.77 & 27.97 & 134.84 & 2310.17 & 49.07 \\
& $\tau_{\theta}^{(0)}$ & age & 106.45 & 83.48 & 17.46 & 84.68 & 323.61 & 47.18 \\
& $\tau_{\gamma}^{(0)}$ & time & 20.50 & 13.88 & 4.45 & 17.17 & 56.53 & 11.25 \\
& $\tau_{\xi}^{(0)}$ & space & 4.06 & 0.65 & 2.86 & 4.04 & 5.41 & 4.03 \\
& $\tau_{\delta}^{(0)}$ & space-time & 10.81 & 2.21 & 7.21 & 10.56 & 15.85 & 10.04 \\
& $\phi^{(0)}$ & mixing & 0.94 & 0.06 & 0.79 & 0.96 & 1.00 & 0.99 \\
\hline \multirow{5}{*}{ Poisson } & $\tau_{\epsilon}^{(1)}$ & error & 1.66 & 0.01 & 1.64 & 1.66 & 1.68 & 1.65 \\
& $\tau_{\theta}^{(1)}$ & age & 147199 & 2346796 & 542.34 & 12478 & 919535 & 1052 \\
& $\tau_{\gamma}^{(1)}$ & time & 1633 & 1953 & 160.96 & 1047 & 6652 & 425.62 \\
& $\tau_{\xi}^{(1)}$ & space & 139.19 & 33.79 & 86.90 & 134.31 & 218.58 & 124.76 \\
& $\tau_{\delta}^{(1)}$ & space-time & 341.57 & 104.99 & 185.82 & 324.47 & 594.36 & 293.16 \\
& $\phi^{(1)}$ & mixing & 0.50 & 0.22 & 0.11 & 0.51 & 0.89 & 0.53 \\
\hline
\end{tabular}

enough predicators. On the other hand, the precision distribution for the Poisson part shows different patterns. There exist much patient level variations based on the small precision of the error term. The spatial effect still contributes much variation compared to others, but the variation for the temporal or the age effect is rather small. The extreme large precisions for the temporal and age effects suggest little deviation from linear models. In other words, linear models for the age and temporal effect could be sufficient. In the end, the mixing parameter $\phi^{(0)}$ for the spatial effect from the Logistic part is quite skewed to its upper bound 1, which indicate strong structured spatial correlation. However, $\phi^{(1)}$ for the Poisson part is centered around 0.5, which puts approximately equal weights between the random noise and the structured spatial component. 


\subsubsection{Covariate Effects}

\section{Race and stage effects}

To see the effect of race and stage on treatment delay, we generated the box-plot based on the posterior distributions of $\boldsymbol{\beta}^{(m)}$ and $\boldsymbol{\omega}^{(m)}$ in Figure 5.5. For the probability of getting same-day treatment, there is no much difference between white and black, while it varies a lot for patients in different stages. Generally, patients with more advanced stages tended to have smaller probability getting same-day treatment. As for the Poisson regression part, non-white patients had longer delay than whites. Patients with localized or distant stages have less delays than those with regional and In Situ stages.

\section{Age effects}

Recall that we assigned RW2 priors on the age effect $\boldsymbol{\theta}^{(m)}$, which is able to capture the non-linear relationship if exist. Figure 5.6 display the estimates of $\boldsymbol{\theta}^{(m)}$. For the Logistic part, the non-linear relationship is obvious. Patients aged between 50 and 70 have lower chance getting same-day treatment, while chances are higher for younger or older patients. For the Poisson part, a strong linear trend with age is shown. Older patients get treatment quicker than young ones. The strong linear relationship agrees with the extremely high precision estimate of $\tau_{\theta}^{(1)}$.

\section{Temporal effects}

To examine how treatment delay changes over time, we plotted the estimates of $\gamma^{(m)}$ in Figure 5.7. In general, the probability of same-day treatment decreased over time, 
(a) Logistic:Race

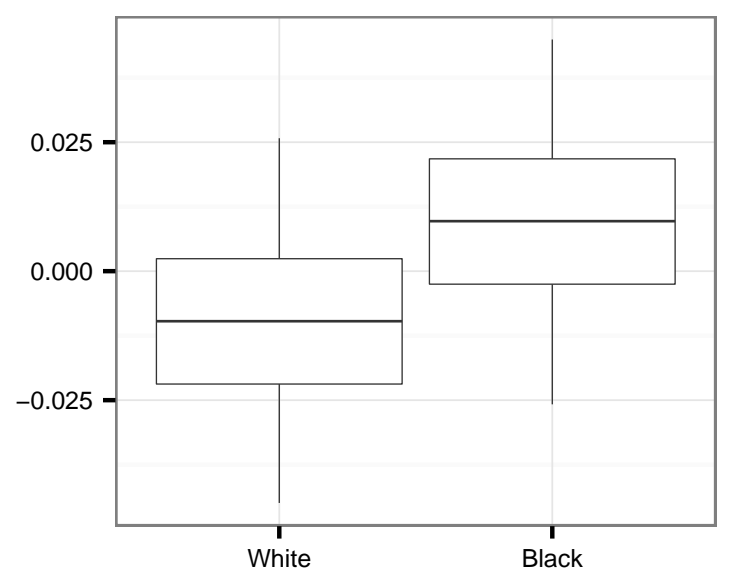

(c) Logistic:Stage

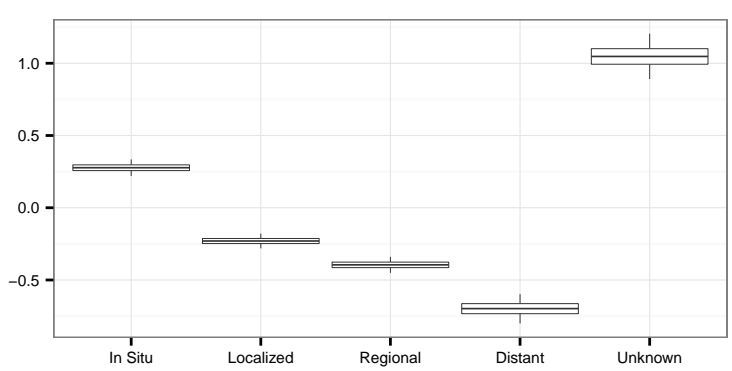

(b) Poisson:Race

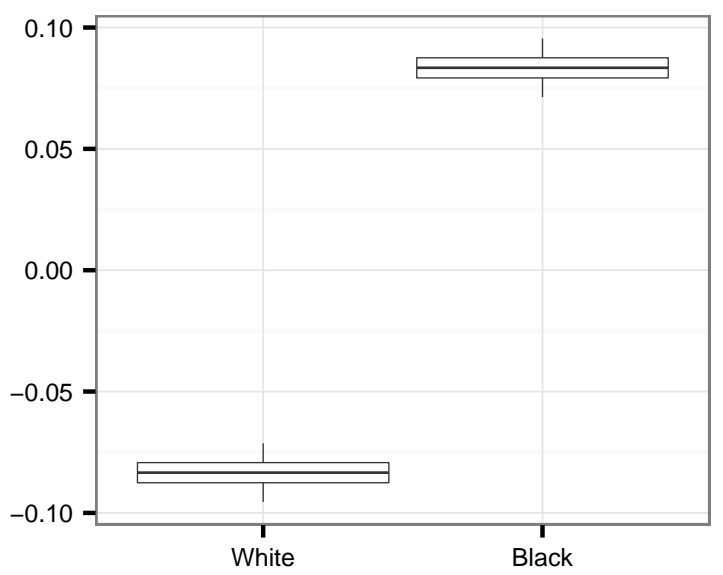

(d) Poisson:Stage

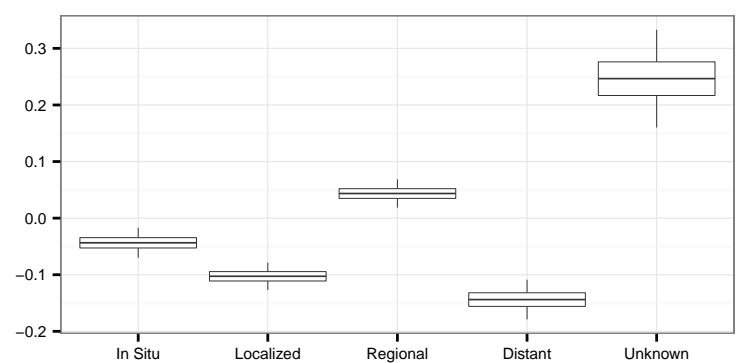

Figure 5.5: Box-plot for the Race and Stage Effects. Note that the lower and upper whisker correspond to the $2.5 \%$ and $97.5 \%$ posterior quantiles. 
(a) Logistic: $\boldsymbol{\theta}^{(0)}$

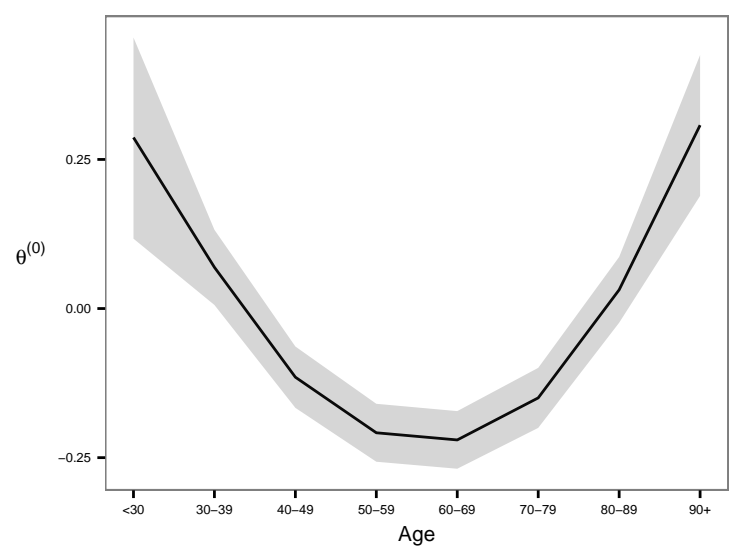

(b) Poisson: $\boldsymbol{\theta}^{(1)}$

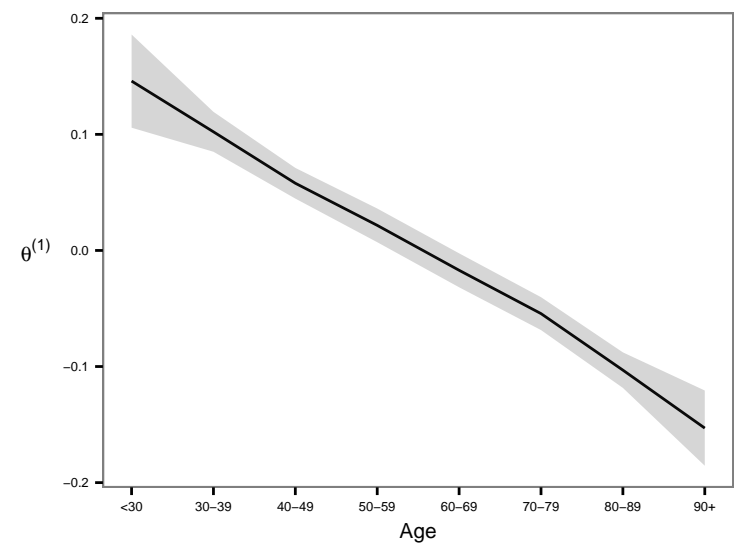

Figure 5.6: Estimates of the Age Effects. The black lines stand for the posterior means of $\boldsymbol{\theta}^{(m)}$ and the shaded gray areas represent the $95 \%$ credible interval.

while the number of delayed days increased over time. The trends were both close to linear, with a little curvature around the boundaries.

\section{Spatial effects}

We plotted the spatial effect $\boldsymbol{\xi}^{(m)}$ on the map of Missouri in Figure 5.8. The spatial pattern is more clear for the Logistic part than the Poisson part. Generally speaking, patients from northwest and southeast have higher probability getting same-day treatment. For the positive delays, patients from south, Kansas city and St. Louis city areas get more delayed.

\section{Spatio-temporal interaction}

Figure 5.9 shows the spatio-temporal interaction effects. We noticed the trends changed over time for both Logistic and Poisson parts. For the Logistic part, red color means higher probability of same-day treatment. The red color starts from the 
(a) Logistic: $\gamma^{(0)}$

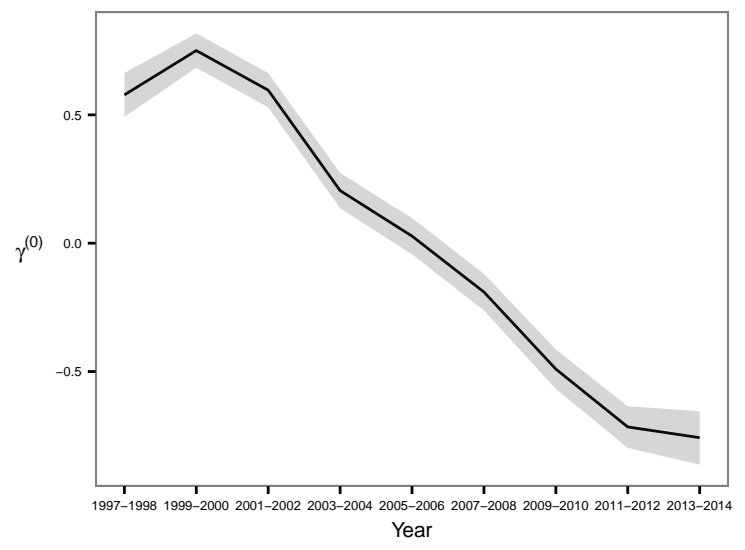

(b) Poisson: $\gamma^{(1)}$

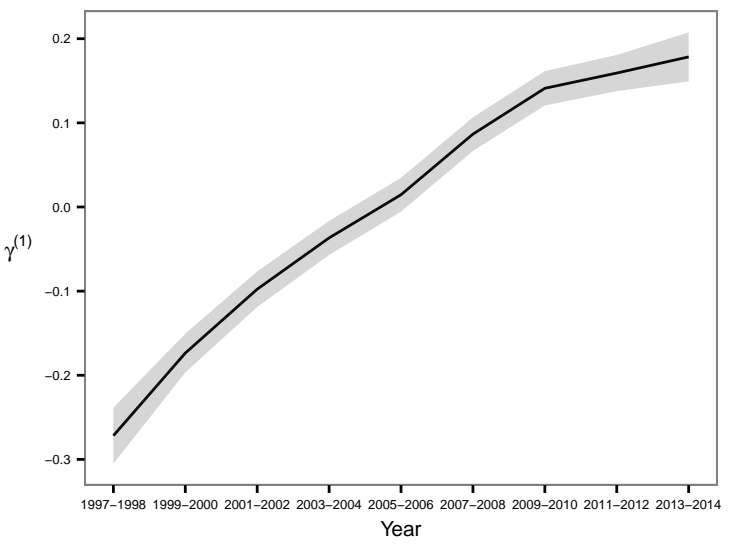

Figure 5.7: Estimates of the Temporal Effects. The black lines stand for the posterior means of $\gamma^{(m)}$ and the shaded gray areas represent the $95 \%$ credible interval.

(a) Logistic: $\boldsymbol{\xi}^{(0)}$

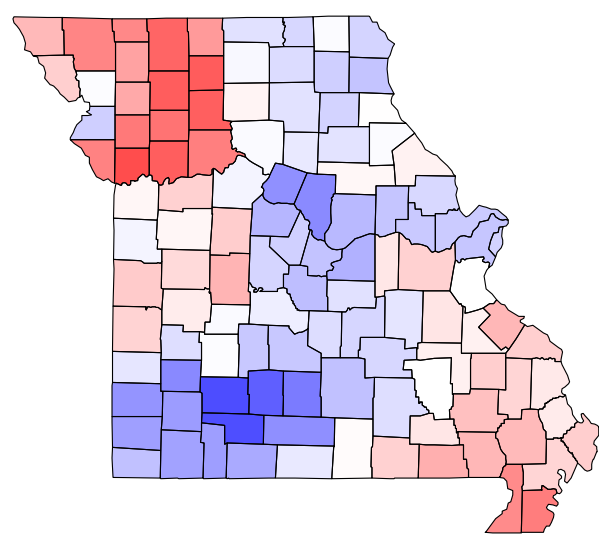

(b) Poisson: $\boldsymbol{\xi}^{(1)}$

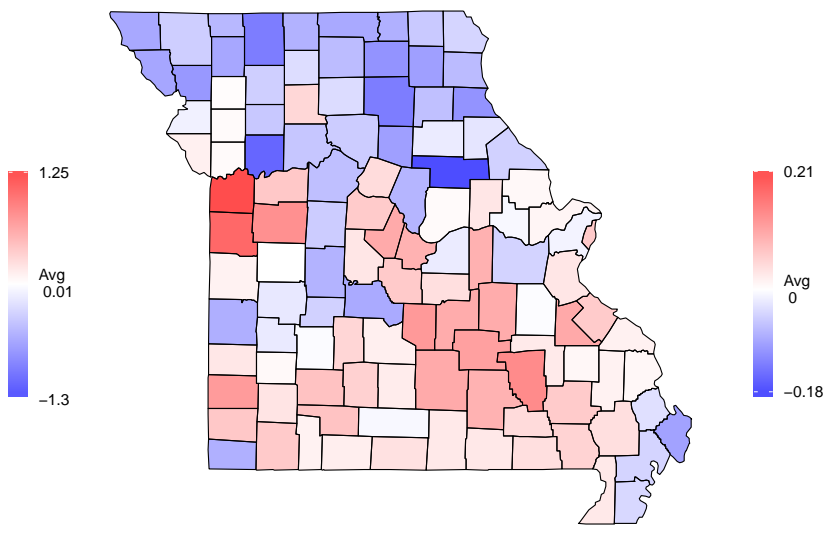

Figure 5.8: Posterior Means of $\boldsymbol{\xi}^{(m)}$. 
west and southeast, then gradually moved to the north and central parts. For the Poisson part, red color means more delayed days. It starts from north and central, then moved to northwest. For recent years, the red mainly shows up in the southwest part.

\subsubsection{Predicted values on the original scale}

In order to visualize the changes of same-day treatment probabilities and delayed days, we plotted the predicted values at the scale of probability and number of days in Figure 5.10. The predictions are only for white patients aged between 60 and 69, diagnosed with localized stage. The patterns in space and time are now easier to see. We can see the decrease of the probability of same-day treatment, along with the increase of delayed days over time. Most of the same-day treatment cases happened in early years and the northwest/southeast part of Missouri. As for the delayed days, the south part of Missouri has more delays than others.

\subsection{Simulation study}

With the huge dimension of our data, MCMC is impossible to give us a nice model fitting result within an acceptable time manner. With the help of INLA, we obtained our results in three days. While INLA is usually very accurate, some rather extreme cases of generalized linear mixed models with binomial or Poisson data have been seen to be problematic (Ferkingstad et al., 2015). Therefore, we conducted two simulations studies to investigate the accuracy of INLA under our model specifications. 
(a) Logistic: $\boldsymbol{\delta}^{(0)}$

NA

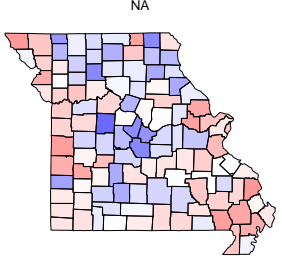

NA

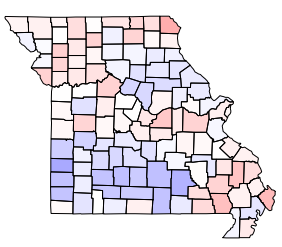

NA

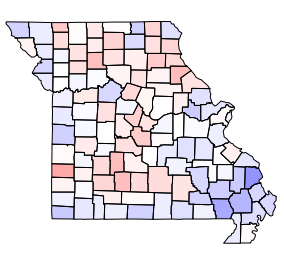

$-1.34$

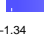

(b) Poisson: $\boldsymbol{\delta}^{(1)}$

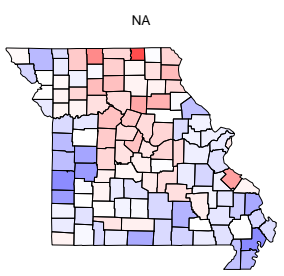

NA

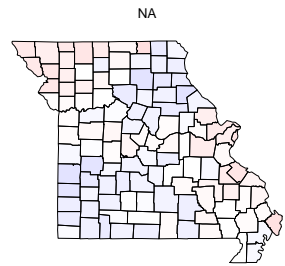

NA

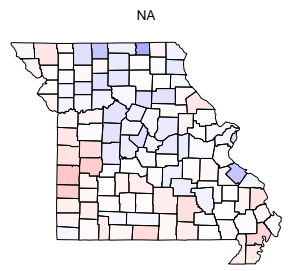

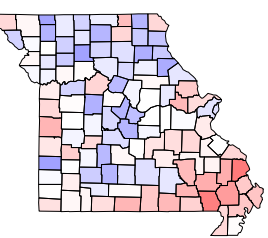

NA

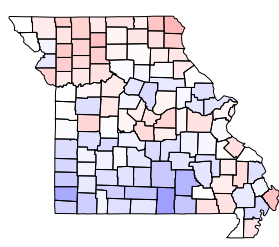

NA

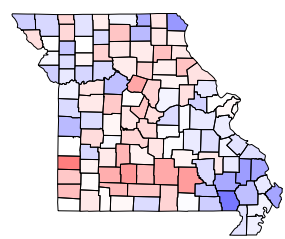

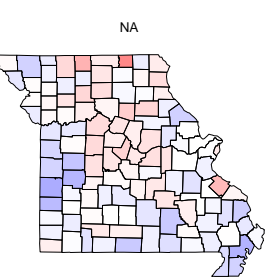
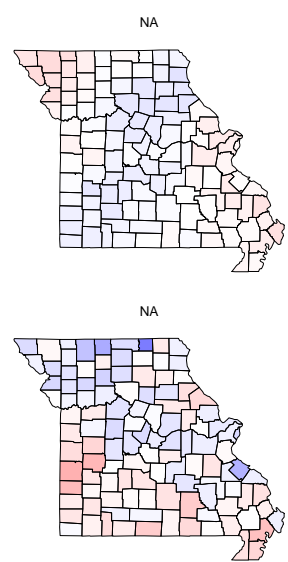

NA

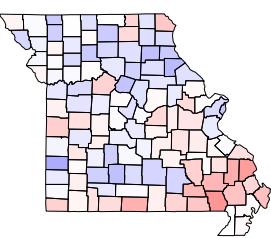

NA

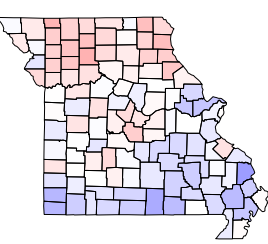

NA

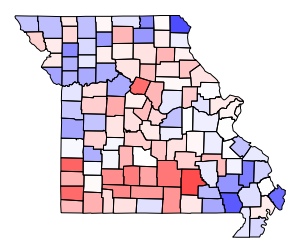

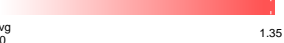

1.35
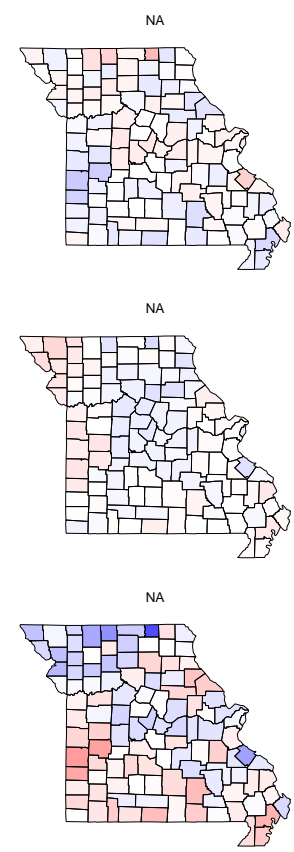

Avg

Figure 5.9: Posterior Means of $\boldsymbol{\delta}^{(m)}$. 
(a) Probability of Zero
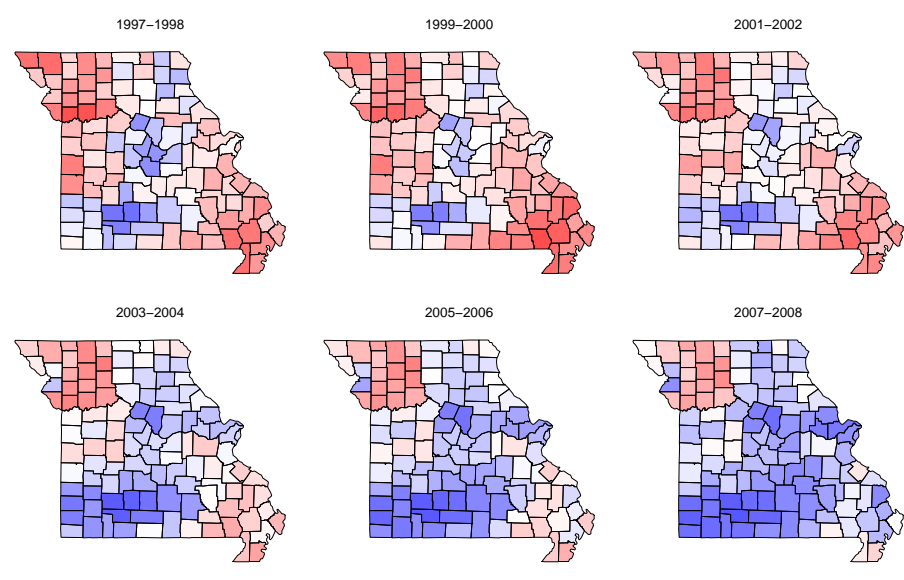

2007-2008
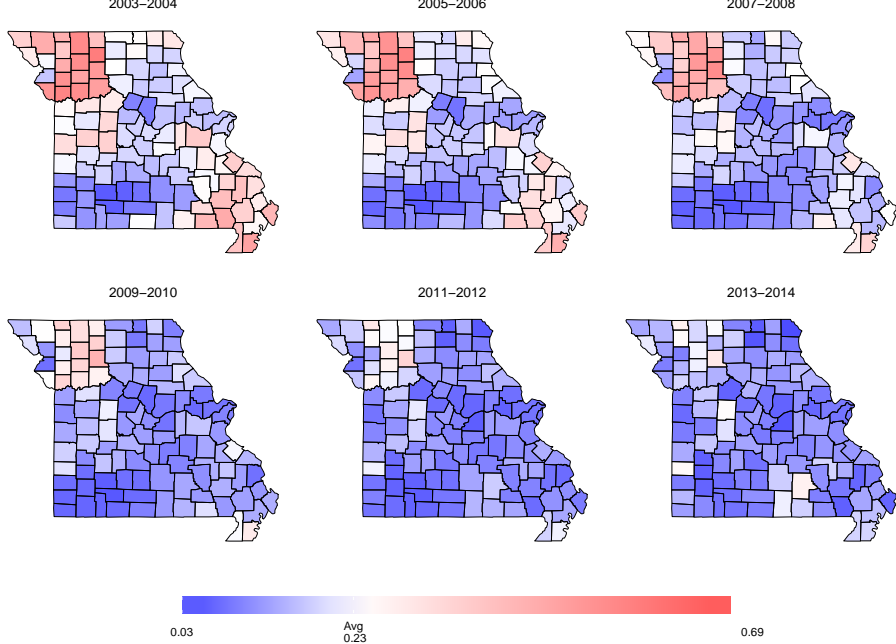

${ }_{0.23}^{\text {Aug }}$

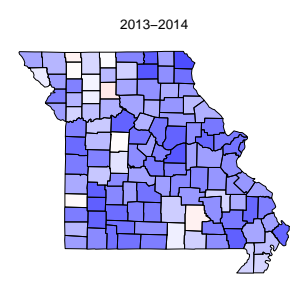

(b) Delayed Days
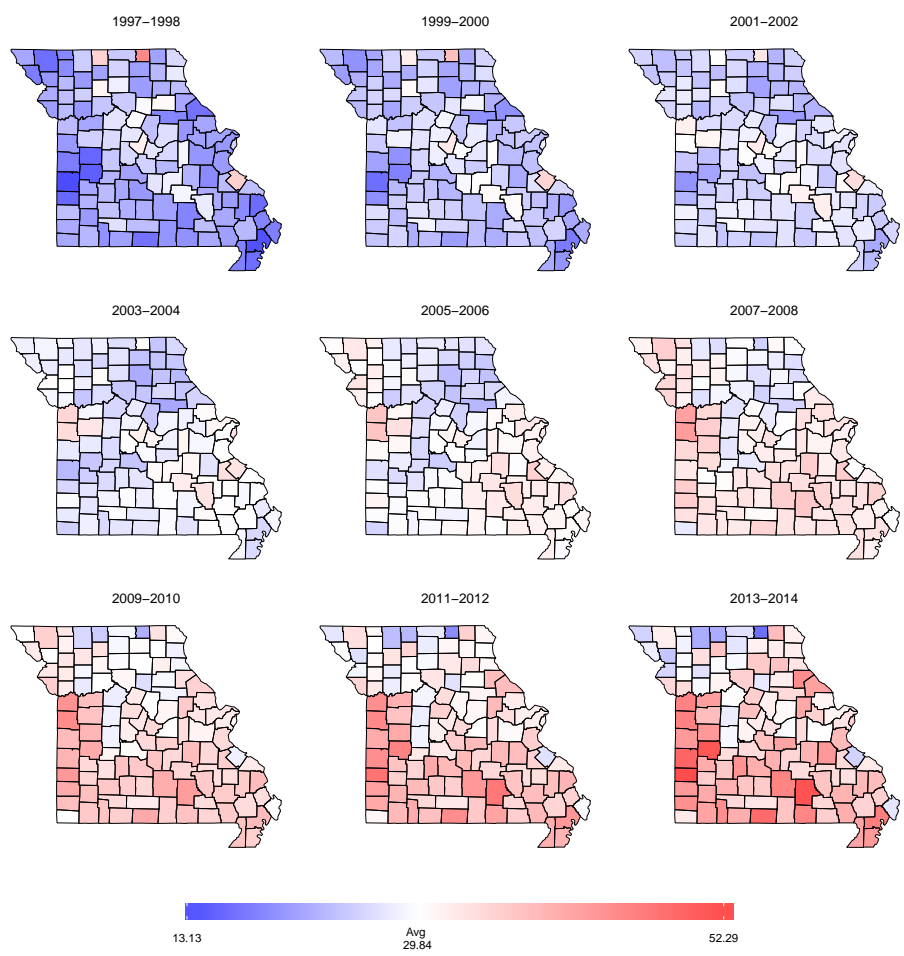

Figure 5.10: Posterior Means of Predicted Same-day Treatment Probabilities and Number of Delayed Days. 


\subsubsection{A balanced simulation}

We want to study if INLA is capable of recovering the true parameter values for fixed effects and random effects. As shown in (5.18) and (5.19), all the covariates in our linear models are categorical variables. We generated treatment delay data $y_{i}$ based on given parameter values for each cross categories. To reduce the model fitting time, we removed the stage effect $\boldsymbol{\omega}^{(m)}$ while only keeping the intercept $\alpha^{(m)}$ and race effect $\boldsymbol{\beta}^{(m)}$. To be specific, given values of hyper parameters $\tau_{\theta}^{(m)}, \tau_{\gamma}^{(m)}, \tau_{\xi}^{(m)}, \tau_{\delta}^{(m)}$ and $\phi^{(m)}$ and fixed effects $\alpha^{(m)}$

1. Form $=1$ and 2 :

- specify values for fixed effect parameters: $\alpha^{(m)}$ and $\boldsymbol{\beta}^{(m)}$.

- specify values for hyper parameters: $\tau_{\theta}^{(m)}, \tau_{\gamma}^{(m)}, \tau_{\xi}^{(m)}, \tau_{\delta}^{(m)}$ and $\phi^{(m)}$.

2. For $m=1$ and 2, simulate $\boldsymbol{\xi}^{(m)}, \boldsymbol{\theta}^{(m)}, \boldsymbol{\gamma}^{(m)}$, and $\boldsymbol{\delta}^{(m)}$ from (5.21), (5.23) and (5.24). Note that $\boldsymbol{\xi}^{(m)}, \boldsymbol{\theta}^{(m)}, \boldsymbol{\gamma}^{(m)}$ and $\boldsymbol{\delta}^{(m)}$ have intrinsic autoregressive (IAR) priors, which are improper. Speckman and Sun (2003) contains the method to simulate from IAR priors. Suppose

$$
\boldsymbol{\psi}_{n} \sim \mathrm{N}_{n}\left(\mathbf{0}, \tau_{\psi}^{-1} \boldsymbol{Q}_{\psi}^{-}\right)
$$

where $\boldsymbol{Q}_{\psi}$ is the precision matrix with $\operatorname{rank} q<n$. Suppose $\boldsymbol{\Gamma}_{n \times q}$ is a matrix with columns being eigenvectors corresponding to positive eigenvalues after the eigendecomposition of $\boldsymbol{Q}_{\psi}$. Let $\boldsymbol{\Lambda}_{q \times q}$ be a diagonal matrix with positive eigenvalues corresponding to $\boldsymbol{\Gamma}_{n \times q}$. Then we have $\boldsymbol{Q}_{\psi}=\boldsymbol{\Gamma}_{n \times q} \boldsymbol{\Lambda}_{q \times q} \boldsymbol{\Gamma}_{n \times q}^{\prime}$. To generate $\boldsymbol{\psi}_{n}$, we first simulate $\boldsymbol{e}_{q} \sim \mathrm{N}_{q}\left(\mathbf{0}, \tau_{\psi}^{-1} \boldsymbol{I}\right)$, then $\boldsymbol{\psi}_{n}=\boldsymbol{\Gamma}_{n \times q} \boldsymbol{\Lambda}_{q \times q}^{1 / 2} \boldsymbol{e}_{q}$ is the sample 
desired, where $\Lambda_{q \times q}^{1 / 2}$ is a diagonal matrix with square-root of the positive eigenvalues on its diagonal. In such way, samples of $\boldsymbol{\xi}^{(m)}, \boldsymbol{\theta}^{(m)}, \boldsymbol{\gamma}^{(m)}$, and $\boldsymbol{\delta}^{(m)}$ can be obtained.

3. For each combination of $q_{1} \in(1,2), q_{2} \in\left(1, \ldots, n_{a}\right), q_{3} \in(1, \ldots, K)$, and $q_{4} \in$ $(1, \ldots, T)$, calculate

$$
\eta_{q_{1} q_{2} q_{3} q_{4}}^{(m)}=\alpha^{(m)}+\beta_{q_{1}}^{(m)}+\theta_{q_{2}}^{(m)}+\xi_{q_{3}}^{(m 0)}+\gamma_{q_{4}}^{(m)}+\delta_{q_{3} q_{4}}^{(m)}+\epsilon_{q_{1} q_{2} q_{3} q_{4}}^{(m)}
$$

where $\epsilon_{q_{1} q_{2} q_{3} q_{4}}^{(m)} \sim \mathrm{N}_{N}\left(\mathbf{0}, \frac{1}{\tau_{\epsilon}^{(m)}} \boldsymbol{I}\right)$ and $m=1,2$.

4. Simulate $N_{s}$ copies of data from HP model. Repeat the following process for $N_{s}$ times.

- For each value of $q_{1} q_{2} q_{3} q_{4}$ :

- Generate $z \sim \operatorname{Bernoulli}\left(\frac{1}{1+\exp \left(-\eta_{q_{1} q_{2} q_{3} q_{4}}^{(0)}\right)}\right)$.

- If $z=1$, set $y_{q_{1} q_{2} q_{3} q_{4}}=0$. If $z=0$, sample $y_{q_{1} q_{2} q_{3} q_{4}} \sim \operatorname{Poisson}\left(\exp \left(\eta_{q_{1} q_{2} q_{3} q_{4}}^{(1)}\right)\right)$.

Notice that if zero was obtained from the Poisson distribution, we need to repeat the Poisson sampling process until we have $y_{q_{1} q_{2} q_{3} q_{4}}>0$.

In the simulation process above, we only generated one set of random effects $\boldsymbol{\xi}^{(m)}, \boldsymbol{\theta}^{(m)}, \boldsymbol{\gamma}^{(m)}$, and $\boldsymbol{\delta}^{(m)}$ as true values along with specified fixed effects $\alpha^{(m)}$ and $\boldsymbol{\beta}^{(m)}$. Using the same set of true parameter values, we generated $N_{s}$ copies of data. For each data set, we generated only one observation for each cross category, which gave us $2 \times n_{a} \times K \times T=2 \times 8 \times 115 \times 9=16560$ observations.

We simulated $N_{s}=30$ treatment delay data sets based on the following true 
values:

$$
\begin{aligned}
& \alpha^{(0)}=-0.9, \quad \boldsymbol{\beta}^{(0)}=(-0.03,0.03)^{\prime} \quad \alpha^{(1)}=3.3, \quad \boldsymbol{\beta}^{(1)}=(-0.1,0.1)^{\prime}, \\
& \tau_{\epsilon}^{(0)}=60, \quad \tau_{\theta}^{(0)}=46, \quad \tau_{\gamma}^{(0)}=11, \quad \tau_{\xi}^{(0)}=4, \quad \tau_{\delta}^{(0)}=11, \quad \phi^{(0)}=0.6, \\
& \tau_{\epsilon}^{(1)}=40, \quad \tau_{\theta}^{(1)}=1100, \quad \tau_{\gamma}^{(1)}=420, \quad \tau_{\xi}^{1)}=125, \quad \tau_{\delta}^{(1)}=270, \quad \phi^{(1)}=0.3 .
\end{aligned}
$$

We used INLA to fit the simulated data with the same computation environment as in Section 5.4. We used the same $\left(U_{0}, a\right)$ and $\left(U_{\phi}, a\right)$ values in Section 5.3.5 for the PC priors of precision parameters in the Logistic regression and mixing parameters. However, for the precision parameters in the Poisson regression, we picked $\left(U_{1}, a=\right.$ $0.01)$ s.t.

$$
0.31 U_{1}=\log \left(y_{\max }\right) / 2 / 1.96
$$

followed by the strategy described in Section 5.4. Each run took approximately 90 minutes. Table 5.2 compares the fixed effects estimates with their true values. The average posterior means of the fixed effects from our 30 simulated data set are close to the true values with small standard deviation. It shows INLA did a good job to recover the true values for the fixed effects. Figure 5.11 shows the scatter plots of the true random effects and their average posterior means. In general, all points are close to the diagonal line, which shows the estimates are close to the true values. The random effects in the Poisson regression part had better estimates than the Logistic part, since points in Figure 5.11b are more concentrated around the diagonal line than those in Figure 5.11a. For both Logistic and Poisson regressions, the spatio-temporal interaction effects $\boldsymbol{\delta}^{(m)}$ deviated from the diagonal lines most, with 
overestimates for smaller values and under estimates for larger values. Figure 5.12 displays the Mean Absolute Deviances (MAD) from the average posterior means to the true values for random effects. The MAD for both Logistic and Poisson regressions show the same patterns, where the age effect $\boldsymbol{\theta}^{(m)}$ and temporal effect $\boldsymbol{\gamma}^{(m)}$ have smaller MAD, followed by the spatial effect $\boldsymbol{\xi}^{(m)}$ and then the spatio-temporal interaction effect $\boldsymbol{\delta}^{(m)}$. Notice that both MAD for $\boldsymbol{\delta}^{(m)}$ have an "U" shape. Recall that $\boldsymbol{\delta}^{(m)}=\left(\delta_{11}^{(m)}, \ldots, \delta_{K 1}^{(m)}, \ldots, \delta_{1 T}^{(m)}, \ldots, \delta_{K T}^{(m)}\right)^{\prime}$ is arranged by year with counties nested in. It shows that the estimates for $\boldsymbol{\delta}^{(m)}$ have larger deviations from true values at the boundary years, i.e. the early years and late years. Table 5.3 contains the estimates for our hyper parameters. For each generated data set, we calculated the mean, standard deviation, $2.5 \%$ quantile, median, $97.5 \%$ quantile and the mode for each hyper parameters. The statistics were averaged across all 30 results, as displayed in the table. Numbers in parenthesis are the standard deviations for the corresponding statistics. Since posterior distribution for precision parameters were highly skewed, it is hard to define a good point estimate. Overall, INLA gave reasonable posterior distributions around our true precision parameter values. There is a little overestimate for the mixing parameter $\phi^{(0)}$, while $\phi^{(1)}$ has better estimates.

Table 5.2: Truth vs. Estimates on Fixed Effects. The mean is calculated as the average estimates from our generated $N_{s}=30$ data sets, while $\mathrm{Sd}$ is the standard deviation of those 30 estimates.

\begin{tabular}{l|c|c|cc}
\hline Model & Para. & Truth & Mean & Sd \\
\hline \multirow{4}{*}{ Logistic } & $\alpha^{(0)}$ & -0.9 & -0.910 & 0.023 \\
& $\beta_{1}^{(0)}$ & -0.03 & -0.038 & 0.016 \\
& $\beta_{2}^{(0)}$ & 0.03 & 0.038 & 0.016 \\
\hline \multirow{3}{*}{ Poisson } & $\alpha^{(1)}$ & 3.300 & 3.300 & 0.002 \\
& $\beta_{1}^{(1)}$ & -0.1 & -0.100 & 0.002 \\
& $\beta_{2}^{(1)}$ & 0.1 & 0.100 & 0.002 \\
\hline
\end{tabular}


(a) Logistic

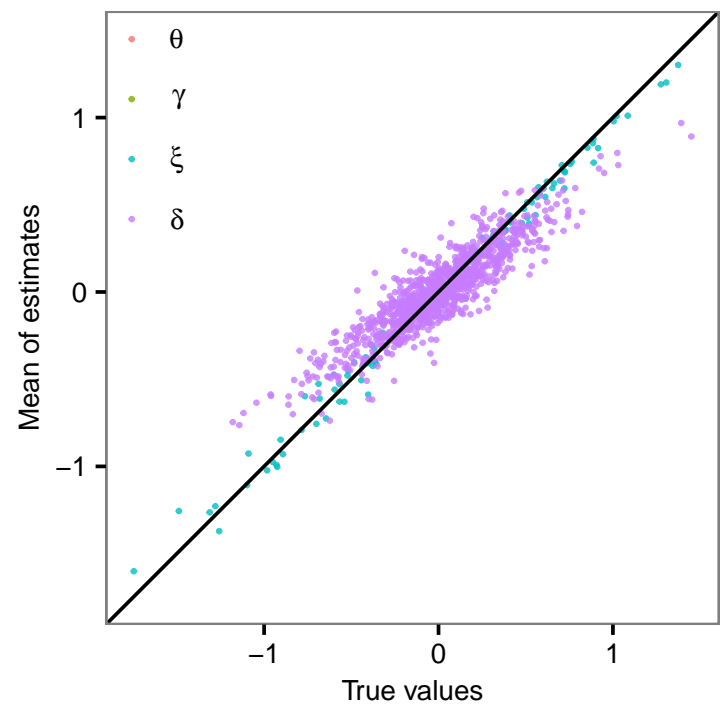

(b) Poisson

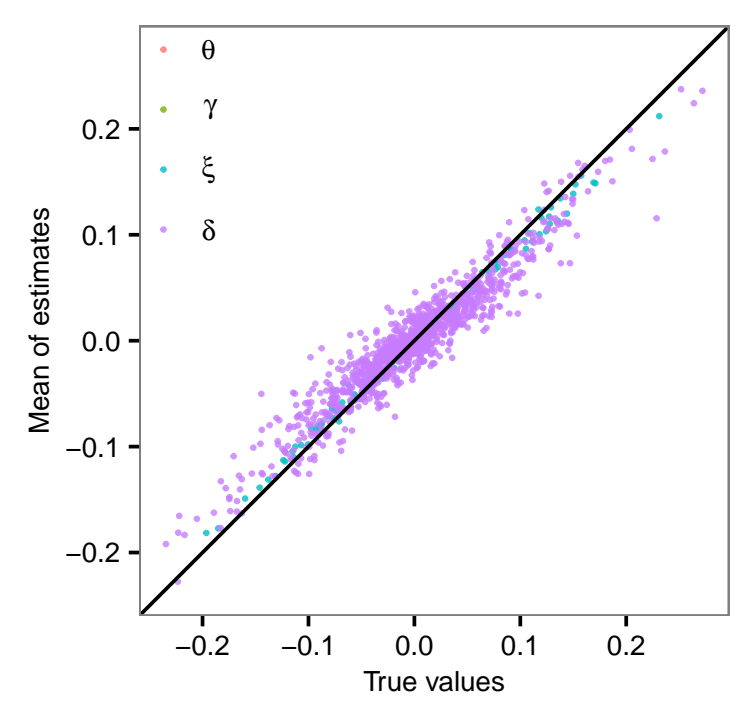

Figure 5.11: Scatter Plots for True vs. Estimates for Random Effects.

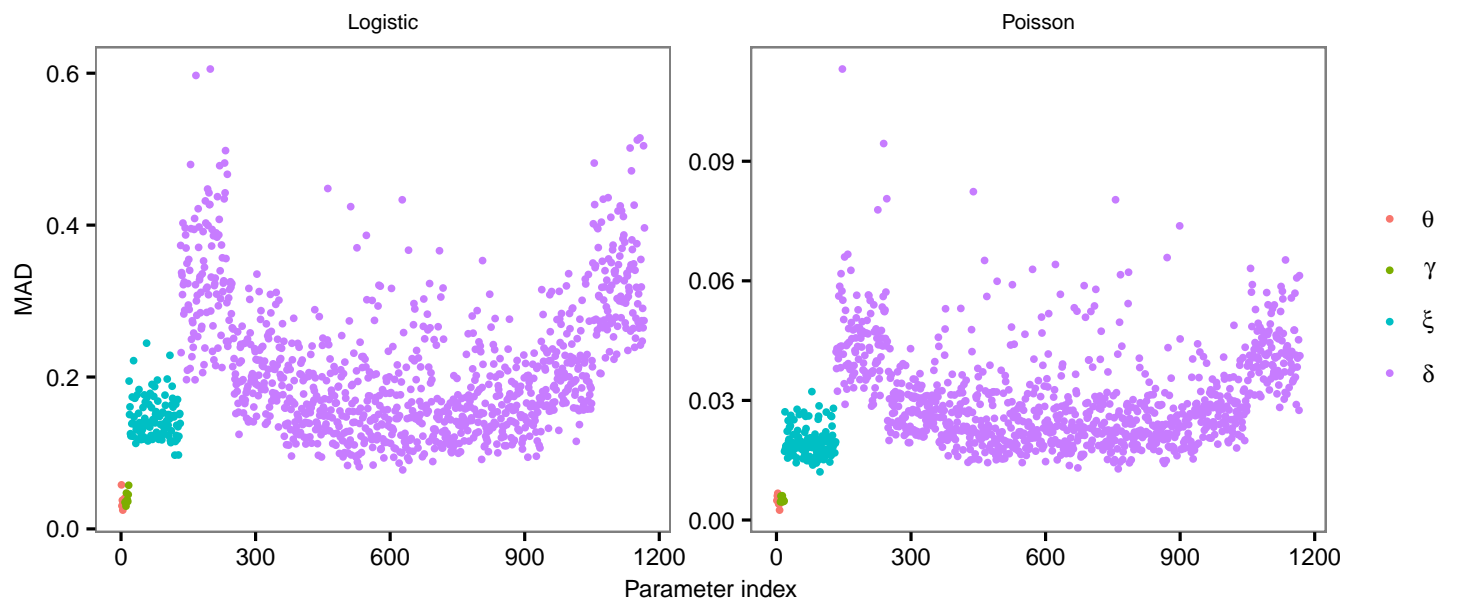

Figure 5.12: Mean Absolute Deviance (MAD) from Estimates to True Values for Random Effects. 


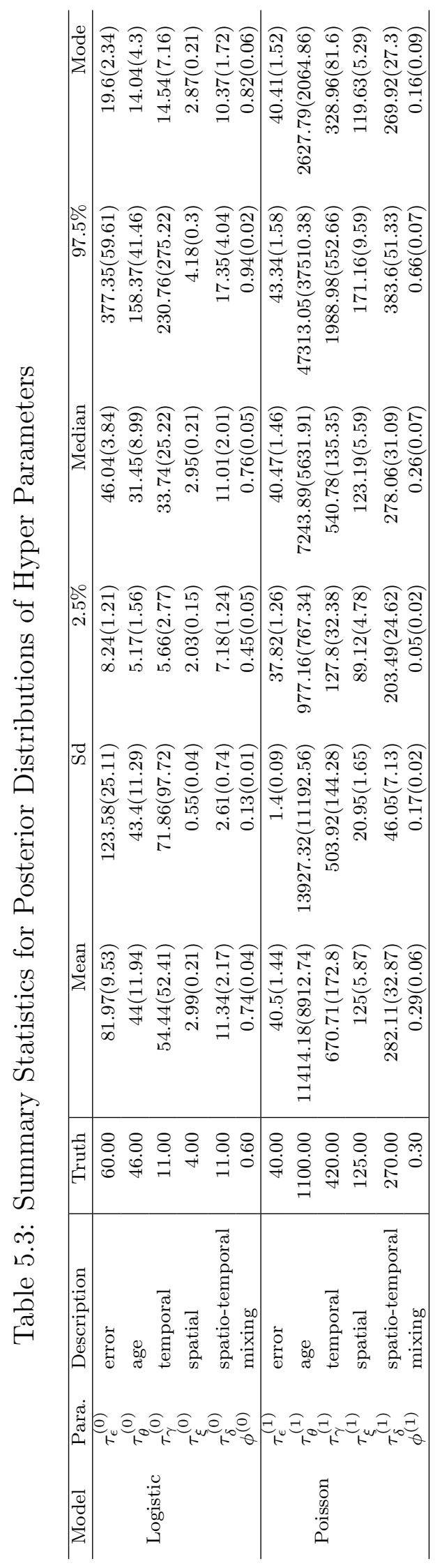




\subsubsection{A matched simulation}

The results in Section 5.5.1 indicate INLA can recover the true parameters values under the balanced setting where every cross category has at one observation. However, this is not the case in our read data analysis. For the read data analysis, there were 73,551 observations. On the other hand, we have 2 categories for race, 5 categories for stage, 8 categories for age, 115 categories for county and 9 categories for year. Therefore, we have $2 \times 5 \times 8 \times 115 \times 9=82800$ cross categories in total. Our 73,551 observations only make up $18.2 \%$ of those categories. In addition, most observations were came from city areas, like St. Louis and Kansas City areas. With such unbalanced data set, we are interested in if INLA can still recover the true parameter values. We simulate our data using the procedure below.

1. For $m=1$ and 2 :

- specify values for fixed effect parameters: $\alpha^{(m)}$ and $\boldsymbol{\beta}^{(m)}$.

- specify values for hyper parameters: $\tau_{\theta}^{(m)}, \tau_{\gamma}^{(m)}, \tau_{\xi}^{(m)}, \tau_{\delta}^{(m)}$ and $\phi^{(m)}$.

2. For $m=1$ and 2, simulate $\boldsymbol{\xi}^{(m)}, \boldsymbol{\theta}^{(m)}, \boldsymbol{\gamma}^{(m)}$, and $\boldsymbol{\delta}^{(m)}$ from (5.21), (5.23) and $(5.24)$.

3. For $i=1, \ldots, N$, where $N$ is the total number of observations in the read data and $i$ refers to the $i^{\text {th }}$ patient, calculate $\eta_{i}^{(0)}$ and $\eta_{i}^{(1)}$ based on (5.18) and (5.19). Notice that for observation $i, r_{i}, s_{i}, a_{i}, c_{i}$ and $t_{i}$ are the corresponding race, stage, age, county and year category.

4. Simulate treatment delay $\boldsymbol{y}=\left(y_{1}, \ldots, y_{N}\right)^{\prime}$ : for $i=1, \ldots, N$ :

- Generate $z \sim \operatorname{Bernoulli}\left(\frac{1}{1+\exp \left(-\eta_{i}^{(0)}\right)}\right)$. 
- If $z=1$, set $y_{i}=0$. If $z=0$, sample $\left.y_{i} \sim \operatorname{Poisson}\left(\exp \left(\eta_{i}^{(1)}\right)\right)\right)$. Notice that if zero was obtained from the Poisson distribution, we need to repeat the Poisson sampling process until we have $y_{i}>0$.

In the end, we obtained 73,551 observations having exactly the same data structure as our read data set. The following true values were used in our simulation:

$$
\begin{aligned}
& \alpha^{(0)}=-0.9, \quad \boldsymbol{\beta}^{(0)}=(-0.01,0.01)^{\prime} \quad \boldsymbol{\omega}^{(0)}=(0.27,-0.23,-0.39,-0.71,1.06)^{\prime}, \\
& \alpha^{(1)}=3.3, \quad \boldsymbol{\beta}^{(1)}=(-0.08,0.08)^{\prime}, \quad \boldsymbol{\omega}^{(0)}=(-0.04,-0.10,0.05,-0.14,0.23)^{\prime}, \\
& \tau_{\epsilon}^{(0)}=40, \quad \tau_{\theta}^{(0)}=46, \quad \tau_{\gamma}^{(0)}=11, \quad \tau_{\xi}^{(0)}=4, \quad \tau_{\delta}^{(0)}=11, \quad \phi^{(0)}=0.9, \\
& \tau_{\epsilon}^{(1)}=100, \quad \tau_{\theta}^{(1)}=1100, \quad \tau_{\gamma}^{(1)}=420, \quad \tau_{\xi}^{1)}=125, \quad \tau_{\delta}^{(1)}=270, \quad \phi^{(1)}=0.6 .
\end{aligned}
$$

We used INLA to get the model fitting result, which took around 71 hours. We chose the PC priors using the same rule as in Section 5.5.1. Table 5.4 contains the true values for fixed effects and the posterior summary statistics from INLA. Except for the race effects $\beta_{1}^{(0)}$ and $\beta_{2}^{(0)}$ in the Logistic regression, all other parameters have their true values nicely recovered. For $\beta_{1}^{(0)}$ and $\beta_{2}^{(0)}$, their true values -0.01 and 0.01 while the posterior means are -0.043 and 0.043 . The random noise with precision $\tau_{\epsilon}^{(0)}=40$ could be the reason to make small values $\beta_{1}^{(0)}$ and $\beta_{2}^{(0)}$ hard to estimate. Figure 5.13 plots the true and estimated values for age effects. The true values are in red color and highly nonlinear. The blue lines are estimates from INLA with gray ribbon standing for $95 \%$ credible interval. We can see INLA has discovered similar age patterns as the true values. Figure 5.14 shows the time effect. INLA was still capable of capturing the main trends. In addition, we plotted the spatial effect $\boldsymbol{\xi}^{(m)}$ and spatio-temporal 
interaction effect $\boldsymbol{\delta}^{(m)}$ from both Logistic and Poisson regressions in Figure 5.15, 5.16, 5.17 and 5.18. All figures suggest exactly the same patterns between true values and estimates from INLA based on posterior means. In the end, Table 5.5 shows the posterior summary statistics for our hyper parameters. In general, INLA gave us hyper parameter estimates reasonably close to the true values.

We conclude that INLA can produce fairly close estimates of both fixed and random effects to their true values. The deviations can be a result of randomness as well as the inclusion of random noises. It is easier to recover true parameter values from a Poisson regression than a Logistic regression. Overall, conclusion obtained from INLA when analyzing covariate effects are the same as suggested by the true values.

Table 5.4: Truth vs. Estimates on Fixed Effects for Matched Simulation.

\begin{tabular}{l|c|c|cccccc}
\hline Model & Para. & Truth & Mean & Sd & $2.5 \%$ & Median & $97.5 \%$ & Mode \\
\hline \multirow{5}{*}{ Logistic } & $\alpha^{(0)}$ & -0.9 & -0.870 & 0.038 & -0.945 & -0.870 & -0.796 & -0.870 \\
\cline { 2 - 9 } & $\beta_{1}^{(0)}$ & -0.01 & -0.043 & 0.018 & -0.079 & -0.043 & -0.008 & -0.043 \\
& $\beta_{2}^{(0)}$ & 0.01 & 0.043 & 0.018 & 0.007 & 0.043 & 0.079 & 0.043 \\
\cline { 2 - 9 } & $\omega_{1}^{(0)}$ & 0.27 & 0.284 & 0.029 & 0.228 & 0.284 & 0.340 & 0.284 \\
& $\omega_{2}^{(0)}$ & -0.23 & -0.211 & 0.025 & -0.260 & -0.211 & -0.161 & -0.211 \\
& $\omega_{3}^{(0)}$ & -0.39 & -0.382 & 0.028 & -0.436 & -0.382 & -0.328 & -0.382 \\
& $\omega_{4}^{(0)}$ & -0.71 & -0.718 & 0.050 & -0.818 & -0.718 & -0.621 & -0.718 \\
& $\omega_{5}^{(0)}$ & 1.06 & 1.028 & 0.078 & 0.875 & 1.028 & 1.182 & 1.028 \\
\hline \multirow{5}{*}{ Poisson } & $\alpha^{(1)}$ & 3.3 & 3.290 & 0.007 & 3.277 & 3.290 & 3.302 & 3.290 \\
\cline { 2 - 9 } & $\beta_{1}^{(1)}$ & -0.08 & -0.079 & 0.002 & -0.082 & -0.079 & -0.076 & -0.079 \\
& $\beta_{2}^{(1)}$ & 0.08 & 0.079 & 0.002 & 0.076 & 0.079 & 0.082 & 0.079 \\
\cline { 2 - 8 } & $\omega_{1}^{(1)}$ & -0.04 & -0.038 & 0.004 & -0.045 & -0.038 & -0.031 & -0.038 \\
& $\omega_{2}^{(1)}$ & -0.10 & -0.100 & 0.003 & -0.107 & -0.100 & -0.094 & -0.100 \\
& $\omega_{3}^{(1)}$ & 0.05 & 0.044 & 0.003 & 0.038 & 0.044 & 0.051 & 0.044 \\
& $\omega_{4}^{(1)}$ & -0.14 & -0.138 & 0.005 & -0.148 & -0.138 & -0.129 & -0.138 \\
& $\omega_{5}^{(1)}$ & 0.23 & 0.232 & 0.011 & 0.211 & 0.232 & 0.253 & 0.232 \\
\hline
\end{tabular}


(a) Logistic: $\boldsymbol{\theta}^{(0)}$

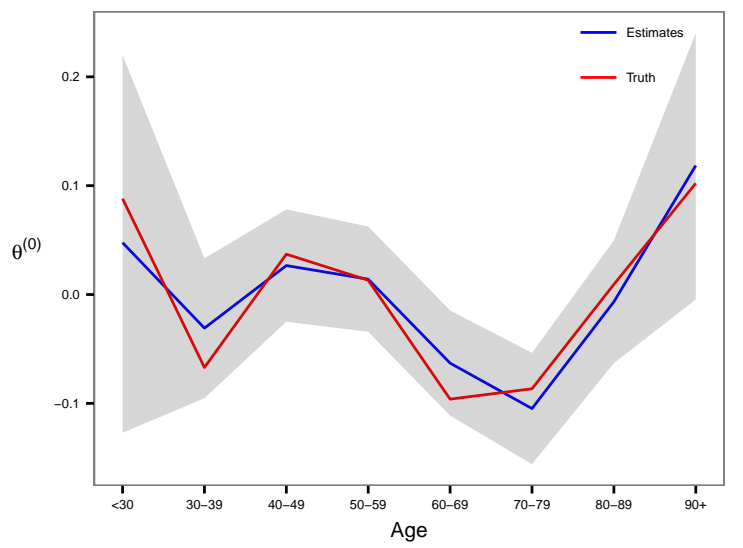

(b) Poisson: $\boldsymbol{\theta}^{(1)}$

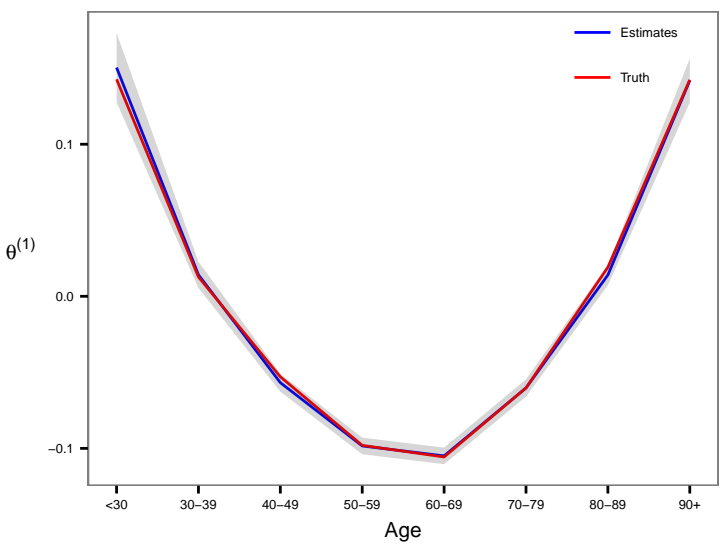

Figure 5.13: Age Effects: Truth vs Estimates. The blue lines stand for the posterior means of $\boldsymbol{\theta}^{(m)}$ and the shaded gray areas represent the $95 \%$ credible interval. The red lines are true values used for simulation.

(a) Logistic: $\gamma^{(0)}$

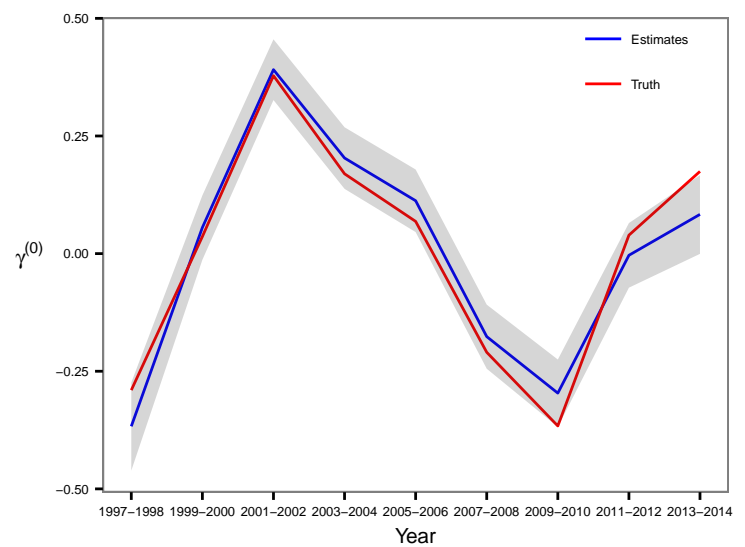

(b) Poisson: $\gamma^{(1)}$

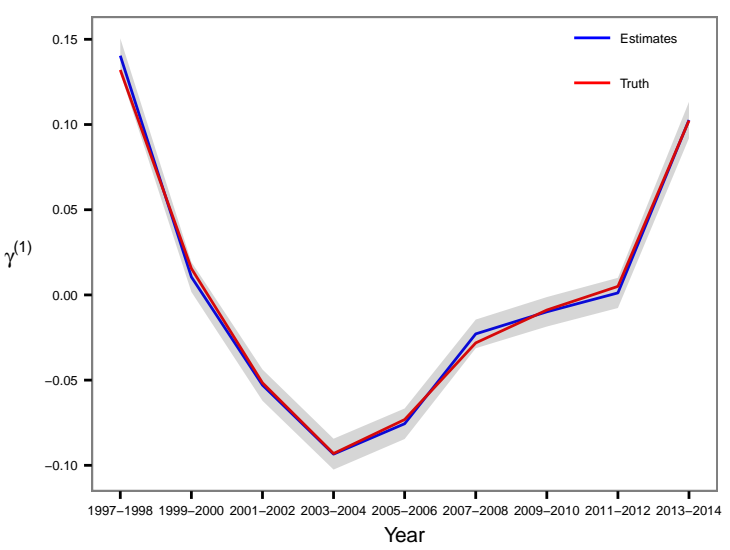

Figure 5.14: Temporal Effects: Truth vs Estimates. The blue lines stand for the posterior means of $\gamma^{(m)}$ and the shaded gray areas represent the $95 \%$ credible interval. The red lines are true values used for simulation. 
(a) Truth

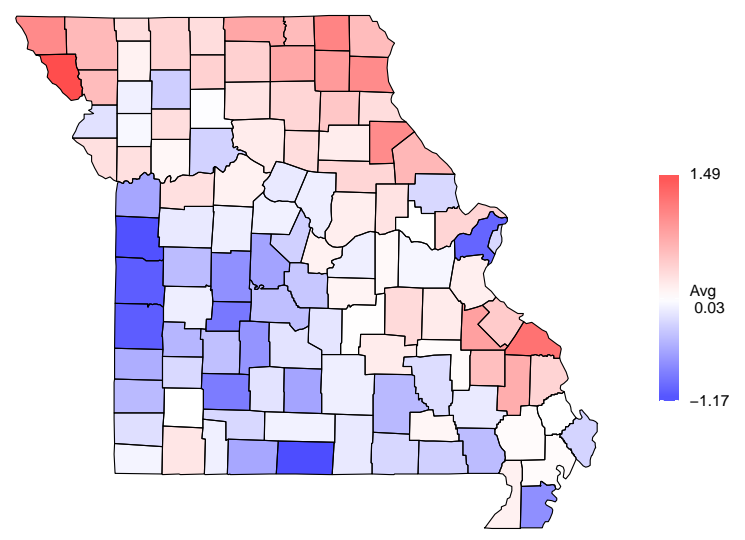

(b) Estimates

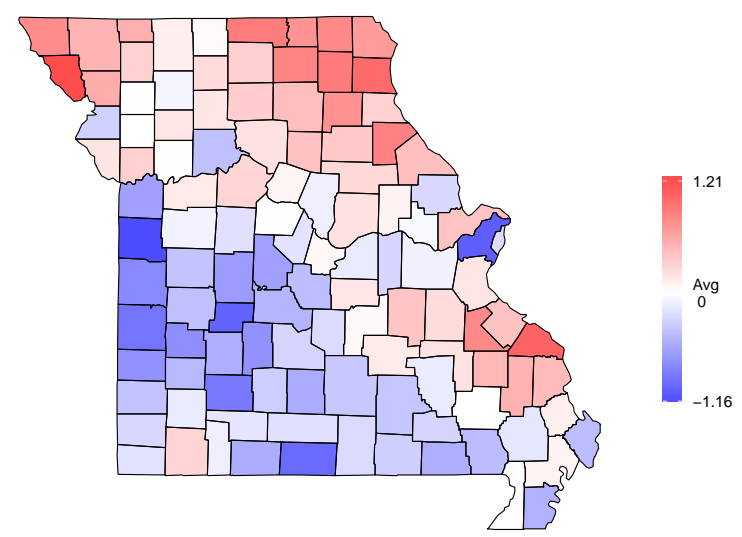

Figure 5.15: Comparison of Estimates for $\boldsymbol{\xi}^{(0)}$ and the True Values Used in Simulation.

(a) Truth

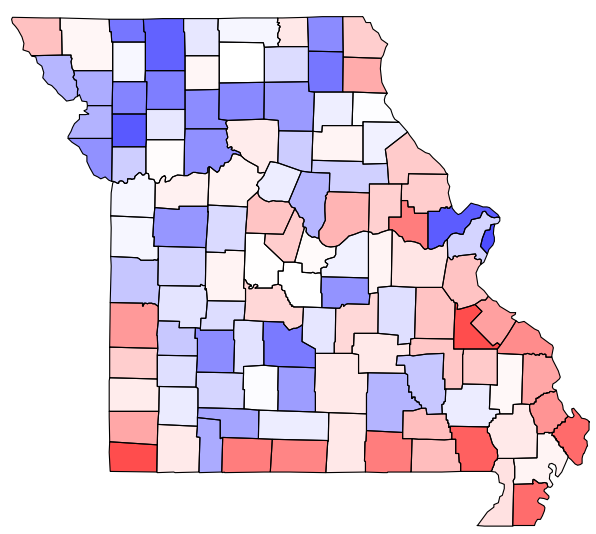

(b) Estimates

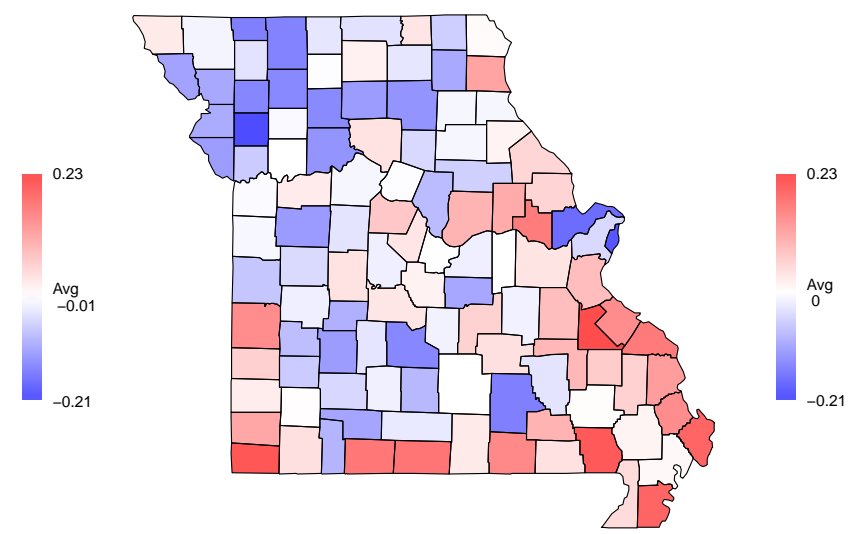

Figure 5.16: Comparison of Estimates for $\boldsymbol{\xi}^{(1)}$ and the True Values Used in Simulation. 
(a) Truth
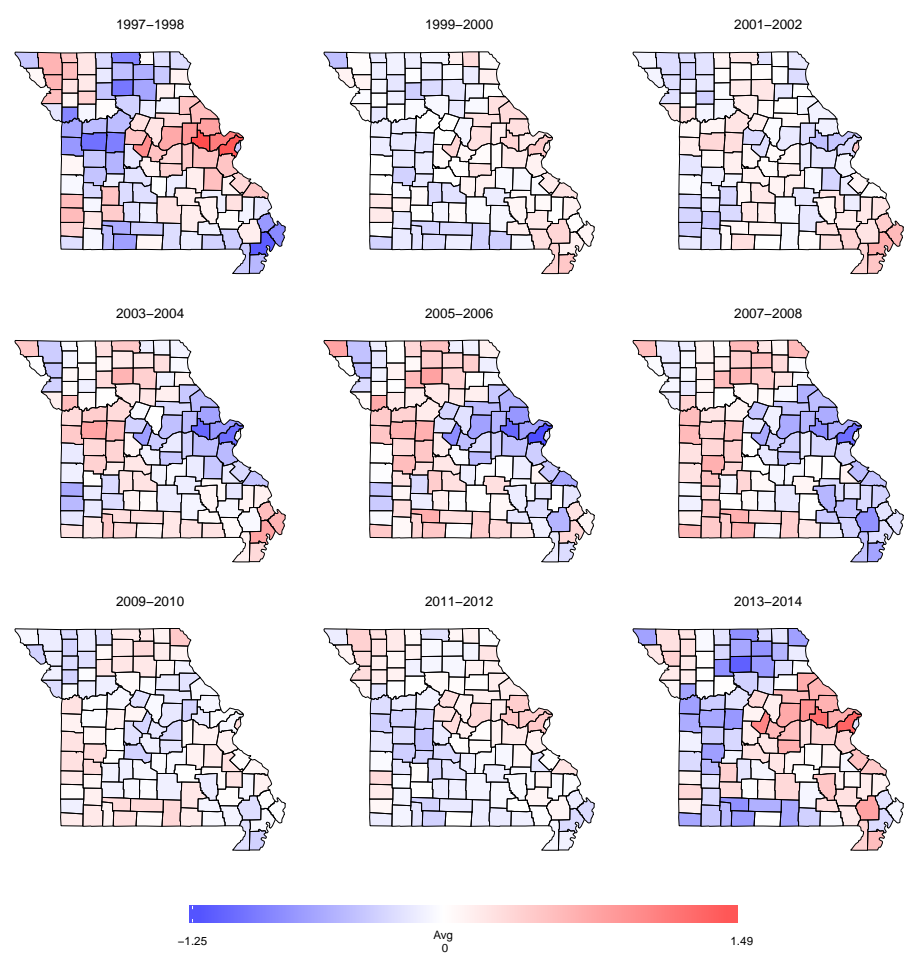

(b) Estimates
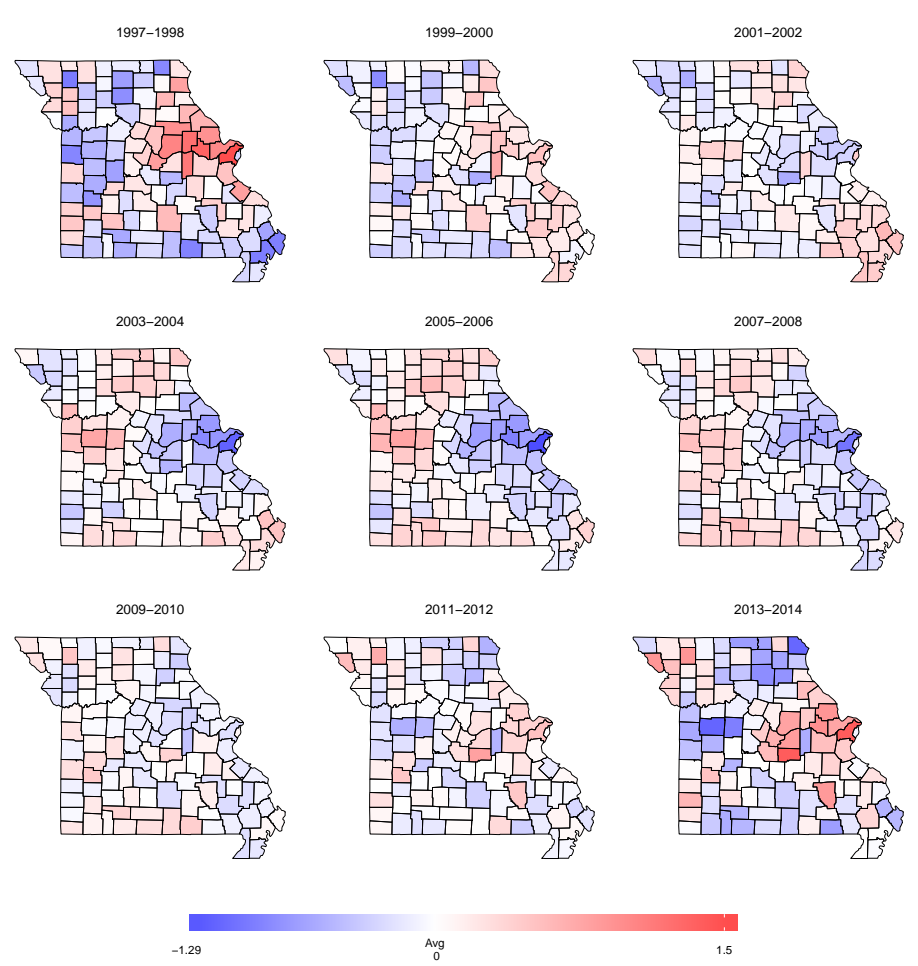

Figure 5.17: Comparison of Estimates for $\boldsymbol{\delta}^{(0)}$ and the True Values Used in Simulation. 
(a) Truth
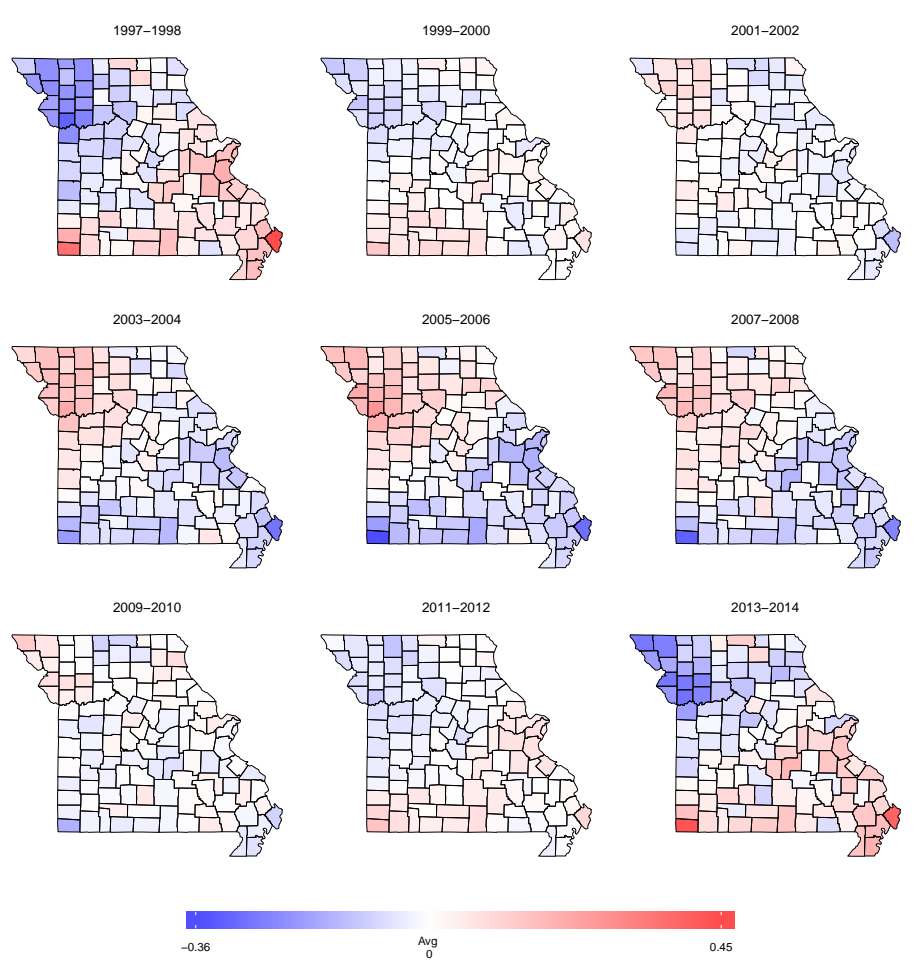

(b) Estimates
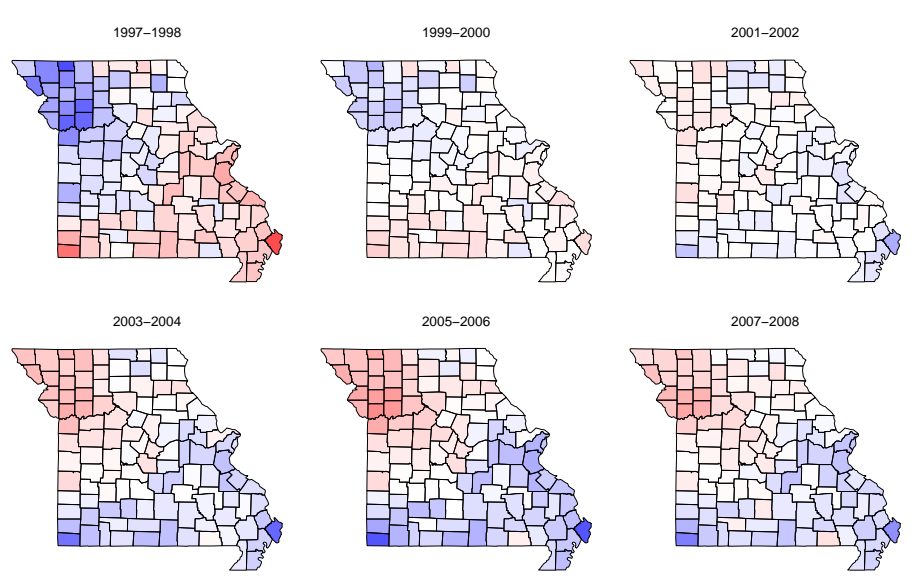

2009-2010
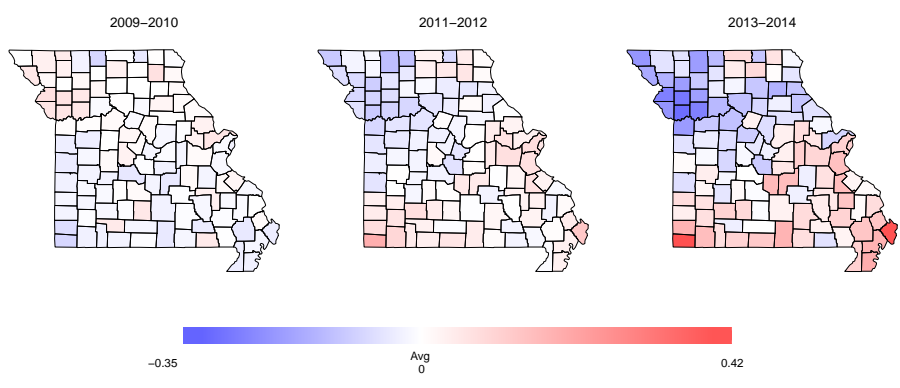

Figure 5.18: Comparison of Estimates for $\boldsymbol{\delta}^{(1)}$ and the True Values Used in Simulation. 
Table 5.5: Summary Statistics for Posterior Distributions of Hyper Parameters in Matched Simulation.

\begin{tabular}{cll|c|cccccc}
\hline Model & Para. & Description & Truth & Mean & Sd & $2.5 \%$ & Median & $97.5 \%$ & Mode \\
\hline \multirow{5}{*}{ Logistic } & $\tau_{\epsilon}^{(0)}$ & error & 40 & 125.24 & 175.62 & 15.77 & 73.50 & 550.51 & 35.18 \\
& $\tau_{\theta}^{(0)}$ & age & 46 & 107.42 & 102.93 & 11.71 & 77.91 & 378.88 & 32.86 \\
& $\tau_{\gamma}^{(0)}$ & time & 11 & 10.61 & 7.03 & 2.52 & 8.91 & 28.88 & 6.07 \\
& $\tau_{\xi}^{(0)}$ & space & 4 & 5.07 & 0.88 & 3.50 & 5.03 & 6.93 & 4.97 \\
& $\tau_{\delta}^{(0)}$ & space-time & 11 & 13.87 & 2.09 & 10.18 & 13.73 & 18.37 & 13.47 \\
& $\phi^{(0)}$ & mixing & 0.9 & 0.90 & 0.08 & 0.68 & 0.92 & 0.99 & 0.98 \\
\hline \multirow{5}{*}{ Poisson } & $\tau_{\epsilon}^{(1)}$ & error & 100 & 101.57 & 3.36 & 95.20 & 101.50 & 108.37 & 101.31 \\
& $\tau_{\theta}^{(1)}$ & age & 1100 & 585.17 & 425.65 & 113.92 & 478.01 & 1691.59 & 294.60 \\
& $\tau_{\gamma}^{(1)}$ & time & 420 & 279.07 & 167.84 & 68.64 & 243.01 & 702.32 & 171.32 \\
& $\tau_{\xi}^{(1)}$ & space & 125 & 108.69 & 19.81 & 74.46 & 107.13 & 152.18 & 104.18 \\
& $\tau_{\delta}^{(1)}$ & space-time & 270 & 310.99 & 37.23 & 243.98 & 308.87 & 390.35 & 304.78 \\
& $\phi^{(1)}$ & mixing & 0.6 & 0.59 & 0.17 & 0.26 & 0.60 & 0.88 & 0.62 \\
\hline
\end{tabular}

\subsection{Discussion}

In this chapter, we used a Hurdle Poisson(HP) model for the breast cancer treatment delay data obtained from Missouri Cancer Registry and Research Center. We investigated the relationship between treatment delay and several covariates, including patients race, age, stage, county and year at diagnosis. Inspired by the BYM2 model, we scaled all the random effects in our model. The scaling enabled us to interpret the hyper parameters more easily. More importantly, the choice of hyper priors were more sensible. We used the PC priors for our hyper parameters. However, one may choose other distributions. The use of INLA made it possible to fit our model. Even though it still took 71 hours to run, it finished a job which is nearly impossible for a regular MCMC algorithm.

In Section 5.4, we analyzed the effects for the covariates in our model. However, conclusions need to be draw with caution when interpreting the results. The model 
itself has no problem, but there exist flaws in the data. The large amount of same-day treatment cases is unbelievable at the first glance. The date of diagnosis is accurate. However, the date for the first treatment can be questionable. For most cases, the first treatment is surgery but the definition of surgery may have been changed. For several years, there had been inconsistencies in how registrars were instructed to handled core needle biopsies when the entire tumor apparently was removed in the procedure. That outcome was surmised when no residual tumor was found in the definitive resection specimen. In these scenarios, the date of the core needle biopsy was entered as the Date of First Surgery because it was the first date the tumor was removed. A separate field was added to record the date of the most definitive primary site surgical procedure. Because the way the dates of surgery were entered, it seemed like larger procedures were being used to diagnose breast cancers rather than core needle biopsies. There is only one data field registries use to report the type of surgical procedure, and the procedure code reflects the cumulative results of all surgeries. Registrars entered the earliest date the tumor was removed and the code for the most definitive primary site surgery. At the same time, core needle biopsies were not entered into the diagnostic procedures field because one procedure would represent 2 events, i.e. diagnostic and therapeutic surgeries. A few years ago the Commission on Cancer ( $\mathrm{CoC}$ ) clarified how the process of tissue diagnosis of breast primaries should be abstracted. Core needle biopsies are not to be used to determine the first date of surgical treatment, even when there is no residual tumor found in the resection specimen. The reason is because core needle biopsies don't have inked surgical margins to assess for tumor involvement. As registrars began to change their abstracting practices for breast primaries, the interval between diagnosis and 
treatment would increase for affected cases.

We have conducted simulation study in Section 5.5 to show that INLA can produce consistent results based on the given parameter values. Although there may exist flaws about the treatment data, the HP model with scaled random effects can still be useful in other fields of study. The scaling idea can be very useful when choosing hyper priors. 


\section{Chapter 6}

\section{Summary and concluding remarks}

In Chapter 2, we studied the CRC incidence and mortality rates, which fall under the broader category of disease mapping. Bayesian hierarchal models have been extensively used for disease mapping problems, from a single disease to multiple diseases. In the multivariate disease mapping literature, most of the models are built for data on the same geographical regions. However, the situation with misaligned regions can happen when there are confidential requirements on the release of the data. To our knowledge, Schmaltz (2012) is the only paper discussing such problems. Its MarCAR and ConCAR models solved our problems but limitations do exist in terms of easy implementation. For example, the parameters in the precision matrices do not have explicit ranges to guarantee positive definiteness of the matrices. Such problem prevent people from using modern Bayesian softwares (Stan, INLA) to implement the model, where the explicit ranges are required as inputs. In addition, although our implementation of MarCAR and ConCAR models was through $\mathrm{C}++$, the computation speed is still rather slow, which may require days for larger data set. This disser- 
tation brings up the misaligned regions problem again and hopefully more attention can be put onto this field. The MarCAR and ConCAR models are good start points but there may exist connections with the traditional multivariate disease mapping models. The most important idea in the MarCAR and ConCAR models is the building of overlapping matrices with outside information like population sizes, while the existing multivariate disease mapping models do not include such information. If the connections between MarCAR/ConCAR and multivariate disease mapping models can be studied and established, one could possibly solve the misaligned regions problem by adapting the rich computation resources in the multivariate disease mapping literature.

In Chapter 3, we focused on estimating county level CRCS prevalences in Missouri. The motivation of this project was trying to establish connections between CRCS and CRC incidence/mortality rates. Since the rates are usually analyzed at county-level, we need county-level CRCS prevalences. However, we don't have enough data solely based on MO-CLS. The use of MO-BRFSS data with SAE methods to produce county-level CRCS prevalence can be important to produce a continuous estimation of changes in CRCS prevalences, both spatially and temporally. The direct causality relationship between CRCS and CRC incidence/mortality rates is hard to study, since the effect of CRCS can be delayed for many years. However, one can compared the spatial-temporal changes between the prevalences and rates and study their correlations. Chapter 3 is an important first step to produce spatial-temporal estimates of CRCS for counties in Missouri, as later work can focus on combining the MO-BRFSS and MO-CLS data together, with the spatial variation borrowed from MO-CLS and the temporal changes borrowed from MO-BRFSS. 
In Chapter 4, we began to study breast cancer data in Missouri. The MCR-ARC maintains a high quality breast cancer survival data base, which is ideal for conducting various kinds of survival analysis. As a starting point, we studied the survival time for breast cancer patients in Missouri aiming to find out if geographical differences exist. Our results indicated the geographical differences is small compared to the patients demographic information and the stages of the cancer. For a more comprehensive study, one can include many other variables in the model. For example, the house income of the patient, the insurance type, etc. Those factors may also play important roles.

In Chapter 5, we studied the breast cancer treatment delay, which is the first comprehensive spatio-temporal analysis on that subject in the literature. The high lights at the technical side are the use of PC priors along with scaled random effect and the use of INLA. The scaling process enabled us to assign hyper priors more intuitively. However, not all models can be scaled. For example, those spatial models in Chapter 2, 3 and 4 contains an extra parameter $\rho$ controlling the strength of spatial autocorrelation. Its presence prevented us from scaling the spatial CAR models. Lastly, using INLA to solve Bayesian hierarchical models is promising. We didn't need to develop our own MCMC algorithm and INLA runs much faster than MCMC, since it can be parallelized. However, the current stage of INLA software not support many more advanced or self-defined models.

Of all the applied experience above, we were impressed by the capacity of Bayesian hierarchical models. The modeling framework is clear and the structure of hierarchical models build complicated correlations between variables in an easy way. However, as most Bayesian analysis, the prior distributions are important to conduct proper 
analysis. Care must be taken especially for the choice of hyper priors, like the prior distributions for variance parameters. Sensitivity analysis should be regularly performed. Although different choices of hyper priors may lead to different conclusions for hyper parameters, the estimates for parameters of interest are usually robust.

The future research may focus on the study of surgery types for breast cancer. As for breast cancer surgery, currently there are two types. One is Breast-conserving Surgery (BCS) and the other one is mastectomy, which removes the whole breast. For early stage breast cancer patients, BCS has be shown to be at least as good as mastectomy, or even better, in terms survival. However, the proportion of the use of mastectomy has been increased over recent years. This is counterintuitive and can be an interesting research topic. We can first check the geographical distribution for both surgeries across counties in Missouri to see if differences exist. Later on, the survival differences can also be studied. 


\section{Appendix A}

\section{Theorem and lemmas}

\section{A.1 Positive definiteness requirements for $\gamma$}

Lemma A.1.1. The matrix $\boldsymbol{M}_{\boldsymbol{z}}$ defined in (2.28) can be decomposed into

$$
\boldsymbol{M}_{\boldsymbol{z}}=\boldsymbol{C}_{I-1}^{-1} \cdots \boldsymbol{C}_{1}^{-1} \boldsymbol{M}\left(\boldsymbol{C}_{1}^{-1}\right)^{\prime} \cdots\left(\boldsymbol{C}_{I-1}^{-1}\right)^{\prime}
$$

where $\boldsymbol{C}_{1}, \boldsymbol{C}_{2}, \ldots, \boldsymbol{C}_{I-1}$ are a series of block lower-triangular matrices and

$$
\left|\boldsymbol{M}_{\boldsymbol{z}}\right|=|\boldsymbol{M}|=\prod_{i=1}^{I}\left|\boldsymbol{M}_{i i}\right|,
$$

where $\boldsymbol{M}_{i i}$ is the ith diagonal block in matrix $\boldsymbol{M}$.

Proof. Let $\boldsymbol{M}^{1}=\boldsymbol{M}_{\boldsymbol{z}}$. Then for $i=1, \ldots, I-1$,

1. Build the lower-triangular matrix $\boldsymbol{C}_{i}$ by placing $I_{J_{1}}$ through $I_{J_{I}}$ along the diagonal blocks and set the lower portion to zeros except the $i^{\text {th }}$ column which has 
blocks $\boldsymbol{N}_{j i}=-\boldsymbol{M}_{j i}^{(i)}\left(\boldsymbol{M}_{i i}^{(i)}\right)^{-1}$.

2. The matrix to use on the next step is then $\boldsymbol{M}^{(i+1)}=\boldsymbol{C}_{i} \boldsymbol{M}^{(i)}$. Rather than fully computing the multiplication we can make use of the fact that only elements $\left(\boldsymbol{M}_{i+1, i+1}^{(i+1)}\right)$ through $\left(\boldsymbol{M}_{I, i+1}^{(i+1)}\right)$ will be needed in the next step of the algorithm.

Finally, set $\boldsymbol{M}=\boldsymbol{M}^{(I)}$, which is a block diagonal matrix. Since $\boldsymbol{C}_{i}$ s are lower block diagonal matrices with ones on the diagonals, their determinants are all ones. Thus we have

$$
\left|\boldsymbol{M}_{\boldsymbol{z}}\right|=\left|\boldsymbol{C}_{I-1}\right|^{-1} \cdots\left|\boldsymbol{C}_{1}\right|^{-1}|\boldsymbol{M}|\left|\left(\boldsymbol{C}_{1}^{-1}\right)^{\prime}\right| \cdots\left|\left(\boldsymbol{C}_{I-1}^{-1}\right)^{\prime}\right|=\prod_{i=1}^{I}\left|\boldsymbol{M}_{i i}\right|
$$

Example A.1.1. A trivariate case.

We consider the case where we have only $I=3$ variables. Starting with the matrix $\boldsymbol{M}^{(1)}=\boldsymbol{M}_{\boldsymbol{z}}$, from Lemma A.1.1

$$
\begin{gathered}
\boldsymbol{M}^{(1)}=\left(\begin{array}{ccc}
\boldsymbol{I}_{J_{1}} & \boldsymbol{\Phi}_{12} & \boldsymbol{\Phi}_{13} \\
\boldsymbol{\Phi}_{12}^{\prime} & \boldsymbol{I}_{J_{2}} & \boldsymbol{\Phi}_{23} \\
\boldsymbol{\Phi}_{13}^{\prime} & \boldsymbol{\Phi}_{23}^{\prime} & \boldsymbol{I}_{J_{3}}
\end{array}\right) \\
\boldsymbol{C}_{1}=\left(\begin{array}{ccc}
\boldsymbol{I}_{J_{1}} & \mathbf{0} & \mathbf{0} \\
-\boldsymbol{\Phi}_{12} & \boldsymbol{I}_{J_{2}} & \mathbf{0} \\
-\boldsymbol{\Phi}_{13} & \mathbf{0} & \boldsymbol{I}_{J_{3}}
\end{array}\right)
\end{gathered}
$$




$$
\begin{gathered}
\boldsymbol{M}^{(2)}=\left(\begin{array}{ccc}
\boldsymbol{I}_{J_{1}} & \boldsymbol{\Phi}_{12} & \boldsymbol{\Phi}_{13} \\
\mathbf{0} & \boldsymbol{I}_{J_{2}}-\left(\boldsymbol{\Phi}_{12}\right)^{\prime} \boldsymbol{\Phi}_{12} & \boldsymbol{\Phi}_{23}-\left(\boldsymbol{\Phi}_{12}\right)^{\prime} \boldsymbol{\Phi}_{13} \\
\mathbf{0} & \left(\boldsymbol{\Phi}_{23}\right)^{\prime}-\left(\boldsymbol{\Phi}_{12}\right)^{\prime} \boldsymbol{\Phi}_{13} & \boldsymbol{I}_{J_{3}}-\left(\boldsymbol{\Phi}_{13}\right)^{\prime} \boldsymbol{\Phi}_{13}
\end{array}\right) \\
\boldsymbol{C}_{2}=\left(\begin{array}{ccc}
\boldsymbol{I}_{J_{1}} & \mathbf{0} & \mathbf{0} \\
\mathbf{0} & \boldsymbol{I}_{J_{2}} & \mathbf{0} \\
\mathbf{0} & \boldsymbol{N}_{23} & \boldsymbol{I}_{J_{3}}
\end{array}\right)
\end{gathered}
$$

where $\boldsymbol{N}_{23}=-\left[\left(\boldsymbol{\Phi}_{23}\right)^{\prime}-\left(\boldsymbol{\Phi}_{12}\right)^{\prime} \boldsymbol{\Phi}_{13}\right]\left[\boldsymbol{I}_{J_{2}}-\left(\boldsymbol{\Phi}_{12}\right)^{\prime} \boldsymbol{\Phi}_{12}\right]^{-1}$, then

$$
\boldsymbol{M}=\boldsymbol{C}_{2} \boldsymbol{C}_{1} \boldsymbol{M}_{\boldsymbol{z}}\left(\boldsymbol{C}_{1}\right)^{\prime}\left(\boldsymbol{C}_{2}\right)^{\prime}
$$

is a block diagonal matrix with diagonal blocks

$$
\left\{\begin{array}{l}
\boldsymbol{M}_{11}=\boldsymbol{I}_{J_{1}}, \\
\boldsymbol{M}_{22}=\boldsymbol{I}_{J_{2}}-\left(\boldsymbol{\Phi}_{12}\right)^{\prime} \boldsymbol{\Phi}_{12}, \\
\boldsymbol{M}_{33}=\boldsymbol{I}_{J_{3}}-\left(\boldsymbol{\Phi}_{13}\right)^{\prime} \boldsymbol{\Phi}_{13}-\left[\left(\boldsymbol{\Phi}_{23}\right)^{\prime}-\left(\boldsymbol{\Phi}_{13}\right)^{\prime} \boldsymbol{\Phi}_{12}\right]\left[\boldsymbol{I}_{J_{2}}-\left(\boldsymbol{\Phi}_{12}\right)^{\prime} \boldsymbol{\Phi}_{12}\right]^{-1}\left[\left(\boldsymbol{\Phi}_{23}\right)-\left(\boldsymbol{\Phi}_{12}\right)^{\prime}\left(\mathbf{\Phi}_{1} \cdot 5\right)\right.
\end{array}\right)
$$

Theorem A.1.2. For the covariance matrix $\boldsymbol{\Sigma}_{\boldsymbol{u}}$ (2.30) in the MarCAR model, or the precision matrix $\Omega_{u}$ (2.32) in the ConCAR mode, their positive definiteness requires

$$
\left|\boldsymbol{M}_{i i}\right|>0, \quad i=1, \ldots, I,
$$

where $\boldsymbol{M}_{i i}$ is defined in (A.2).

Proof. From the definitions of $\boldsymbol{\Delta}$ and $\boldsymbol{A}$, we know they are all positive definite. Thus from (2.30) and (2.32), we only requires $\left|\boldsymbol{M}_{\boldsymbol{z}}\right|>0$. Then the conclusion can be reached from (A.2). 
Example A.1.2. Positive definite conditions for bivariate case.

When $I=2$, the same process can be conducted as in Example A.1.1. We will only have matrix $\boldsymbol{M}$ (A.1) with two diagonal blocks (A.3) and (A.4). From Theorem A.1.2 we need

$$
\left|\boldsymbol{M}_{22}\right|=\left|\boldsymbol{I}_{J_{2}}-\left(\boldsymbol{\Phi}_{12}\right)^{\prime} \boldsymbol{\Phi}_{12}\right|=\left|\boldsymbol{I}_{J_{2}}-\gamma_{12}^{2}\left(\boldsymbol{\Psi}_{12}\right)^{\prime} \boldsymbol{\Psi}_{12}\right|>0 .
$$

To find the range for $\gamma_{12}$, solve

$$
\begin{aligned}
\tau^{*}= & \sup \left(\tau>0,\left|\boldsymbol{I}_{J_{2}}-\tau\left(\boldsymbol{\Psi}_{12}\right)^{\prime} \boldsymbol{\Psi}_{12}\right|>0\right) \\
= & \sup \left(\tau>0,\left|\boldsymbol{I}_{J_{2}}-\tau \boldsymbol{Q}_{21} \boldsymbol{V}_{21} \boldsymbol{Q}_{21}^{\prime}\right|>0\right) \\
& \quad \text { where } \boldsymbol{Q}_{21} \boldsymbol{V}_{21} \boldsymbol{Q}_{21}^{\prime} \text { is the eigen decomposition of }\left(\boldsymbol{\Psi}_{12}\right)^{\prime} \boldsymbol{\Psi}_{12} \\
= & \sup \left(\tau>0,\left|\boldsymbol{I}_{J_{2}}-\tau \boldsymbol{V}_{21}\right|>0\right) \\
= & \lambda_{12, J_{1}}^{-1}
\end{aligned}
$$

where $\lambda_{21, J_{1}}$ is the largest eigenvalue of $\left(\boldsymbol{\Psi}_{12}\right)^{\prime} \boldsymbol{\Psi}_{12}$. Thus positive definiteness will result if

$$
\left|\gamma_{12}\right|<\sqrt{\lambda_{12, J_{1}}^{-1}}
$$

Theorem A.1.3. For multivariate cases in MarCAR or ConCAR model, the condition

$$
\left|\gamma_{i_{1} i_{2}}\right|<\sqrt{\lambda_{i_{1} i_{2}, J_{i_{1}}}^{-1}}
$$

where $\lambda_{i_{1} i_{2}, J_{i_{1}}}$ is the largest eigenvalue of $\left(\boldsymbol{\Psi}_{i_{1} i_{2}}\right)^{\prime} \boldsymbol{\Psi}_{i_{1} i_{2}}$. is a necessary condition to ensure the positive definiteness of $\boldsymbol{\Sigma}_{\boldsymbol{u}}$ (2.30) in the MarCAR model, or the precision matrix $\Omega_{\boldsymbol{u}}$ (2.32) in the ConCAR mode. 
Proof. Theorem A.1.2 requires only matrix $\boldsymbol{M}$ (A.1) to be positive definite. For multiple variables, we can always reorder variable $i_{1}$ and $i_{2}$ to be the first and second variable. Then the second diagonal block $\boldsymbol{M}_{22}$ (A.4) needs to be positive definite, which give the condition in (A.7) as in bivariate cases. Since we have variable $i_{1}$ and $i_{2}$ reordered, the requirement is indeed for $\gamma_{i_{1} i_{2}}$. 


\section{Appendix B}

\section{Full conditional distributions}

\section{B.1 Full conditional distributions in Chapter 2}

First we will introduce the notations used in this section. Suppose $x_{1}$ and $x_{2}$ are two

random variables, we use $\left[x_{1}\right]$ to stand for the density function of $x_{1}$, and $\left[x_{2} \mid x_{1}\right]$ is the conditional density function of $x_{2}$ given $x_{1}$.

Given the model set up in Section 2.2, and the prior for $\omega_{i}$ in equation (2.20), together with

$$
[\boldsymbol{\rho}][\gamma] \propto 1
$$

on their support, the joint distribution of all the data and parameters is

$$
[\boldsymbol{y} \mid \boldsymbol{\eta}]\left[\boldsymbol{\eta} \mid \boldsymbol{\alpha}, \boldsymbol{\beta}, \boldsymbol{u}, \boldsymbol{\sigma}^{2}\right]\left[\boldsymbol{\alpha} \mid \mu_{\alpha}, \sigma_{\alpha}^{2}\right]\left[\boldsymbol{\beta} \mid \mu_{\beta}, \sigma_{\beta}^{2}\right]\left[\boldsymbol{u} \mid \boldsymbol{\omega}, \boldsymbol{\rho}, \boldsymbol{\gamma}, \boldsymbol{\sigma}^{2}\right][\boldsymbol{\omega}][\boldsymbol{\rho}][\boldsymbol{\gamma}]\left[\boldsymbol{\sigma}^{2}\right],
$$


where

$$
\begin{aligned}
& {[\boldsymbol{y} \mid \boldsymbol{\eta}]=\prod_{i=1}^{I} \prod_{t=1}^{T} \prod_{j=1}^{J_{i}}\left\{\left[n_{i t j} \exp \left(\eta_{i t j}\right)\right]^{y_{i t j}} \exp \left(-n_{i t j} \exp \left(\eta_{i t j}\right)\right)\right\}} \\
& {\left[\boldsymbol{\eta} \mid \boldsymbol{\alpha}, \boldsymbol{\beta}, \boldsymbol{u}, \boldsymbol{\sigma}^{2}\right]=\prod_{i=1}^{I} \prod_{t=1}^{T} \prod_{j=1}^{J_{i}}\left\{\frac{1}{\sqrt{2 \pi \sigma_{i}^{2}}} \exp \left(-\frac{\left[\eta_{i t j}-\left(\alpha_{i}+\beta_{i} s_{t}+u_{i j}\right)\right]^{2}}{2 \sigma_{i}^{2}}\right)\right\}} \\
& \boldsymbol{\alpha} \mid \mu_{\alpha}, \sigma_{\alpha}^{2} \sim N_{I}\left(\mu_{\alpha} \mathbf{1}_{I}, \sigma_{\alpha}^{2} \boldsymbol{I}_{I \times I}\right), \\
& \boldsymbol{\beta} \mid \mu_{\beta}, \sigma_{\beta}^{2} \sim N_{I}\left(\mu_{\beta} \mathbf{1}_{I}, \sigma_{\beta} \boldsymbol{I}_{I \times I}\right), \\
& {\left[\boldsymbol{u} \mid \boldsymbol{\omega}, \boldsymbol{\rho}, \boldsymbol{\gamma}, \boldsymbol{\sigma}^{2}\right]=(2 \pi)^{-\frac{J}{2}}|\boldsymbol{\Sigma}|^{\frac{1}{2}} \exp \left(-\frac{1}{2} \boldsymbol{u}^{\prime} \boldsymbol{\Sigma}^{-1} \boldsymbol{u}\right)} \\
& {[\boldsymbol{\omega}]=\prod_{i=1}^{I} \frac{a}{\left(a+\omega_{i}\right)^{2}}, \quad \omega_{i}>0} \\
& {[\boldsymbol{\rho}] \propto 1,} \\
& {[\gamma] \propto 1} \\
& {\left[\boldsymbol{\sigma}^{2}\right] \propto \prod_{i=1}^{I} \frac{1}{\left(\zeta^{2}+\sigma_{i}^{2}\right) \sigma_{i}^{-1 / 2}} .}
\end{aligned}
$$

Thus, the full conditional distributions are:

$$
\begin{gathered}
{[\boldsymbol{\eta} \mid \cdot] \propto \prod_{i=1}^{I} \prod_{t=1}^{T} \prod_{j=1}^{J_{i}}\left\{\exp \left(-\frac{\left[\eta_{i t j}-\left(\alpha_{i}+\beta_{i} s_{t}+u_{i j}\right)\right]^{2}}{2 \sigma_{i}^{2}}\right)+\eta_{i t j} y_{i t j}-n_{i t j} \exp \left(\eta_{i t j}\right)\right\} ;} \\
{[\boldsymbol{\alpha} \mid \cdot] \propto \prod_{i=1}^{I} \phi\left(\alpha_{i} \mid \frac{\tau_{\alpha} \mu_{\alpha}+\tau_{\alpha, i} \mu_{\alpha, i}}{\tau_{\alpha}+\tau_{\alpha, i}}, \frac{1}{\tau_{\alpha}+\tau_{\alpha, i}}\right),}
\end{gathered}
$$

where

$$
\tau_{\alpha}=\frac{1}{\sigma_{\alpha}^{2}}, \quad \sigma_{\alpha, i}^{2}=\frac{\sigma_{i}^{2}}{T J_{i}}, \quad \tau_{\alpha, i}=1 / \sigma_{\alpha, i}^{2}, \quad \mu_{\alpha, i}=\frac{\sum_{t=1}^{T} \sum_{j=1}^{J_{i}}\left(\eta_{i t j}-\beta_{i} s_{t}-u_{i j}\right)}{T J_{i}}
$$


and

$$
\begin{gathered}
\phi(x \mid m, v)=\frac{1}{\sqrt{2 \pi v}} \exp \left\{-\frac{(x-m)^{2}}{2 v}\right\} \\
{[\boldsymbol{\beta} \mid \cdot] \propto \prod_{i=1}^{I} \phi\left(\beta_{i} \mid \frac{\tau_{\beta} \mu_{\beta}+\tau_{\beta, i} \mu_{\beta, i}}{\tau_{\beta}+\tau_{\beta, i}}, \frac{1}{\tau_{\beta}+\tau_{\beta, i}}\right),}
\end{gathered}
$$

where

$$
\begin{gathered}
\tau_{\beta}=1 / \sigma_{\beta}^{2}, \quad \sigma_{\beta, i}^{2}=\frac{\sigma_{i}^{2}}{\sum_{t=1}^{T} J_{i} s_{t}^{2}}, \quad \tau_{\beta, i}=1 / \sigma_{\beta, i}^{2}, \quad \mu_{\beta, i}=\frac{\sum_{t=1}^{T} \sum_{j=1}^{J_{i}} s_{t}\left(\eta_{i t j}-\alpha_{i}-u_{i j}\right)}{\sum_{t=1}^{T} J_{i} s_{t}^{2}} \\
\boldsymbol{u} \mid \cdot \sim N\left(\left(\boldsymbol{\Omega}+\boldsymbol{\Omega}_{p}\right)^{-1} \boldsymbol{\Omega}_{p} \boldsymbol{u}_{p},\left(\boldsymbol{\Omega}+\boldsymbol{\Omega}_{p}\right)^{-1}\right),
\end{gathered}
$$

where

$$
\begin{aligned}
& \boldsymbol{\Sigma}_{p}=\left(\begin{array}{cccc}
\frac{\sigma_{1}^{2}}{T} \boldsymbol{I}_{J_{1}} & 0 & \cdots & 0 \\
0 & \frac{\sigma_{2}^{2}}{T} \boldsymbol{I}_{J_{2}} & \cdots & \vdots \\
\vdots & \vdots & \ddots & 0 \\
0 & \cdots & 0 & \frac{\sigma_{I}^{2}}{T} \boldsymbol{I}_{J_{I}}
\end{array}\right)_{J_{+} \times J_{+}}, \quad \boldsymbol{u}_{p}=\left(\begin{array}{c}
u_{p, 11} \\
u_{p, 12} \\
\vdots \\
u_{p, I J_{i}}
\end{array}\right)_{J_{+} \times 1}, \\
& u_{p, i j}=\frac{\sum_{t=1}^{T}\left(\eta_{i t j}-\alpha_{i}-\beta_{i} s_{t}\right)}{T}, \quad \Omega_{p}=\Sigma_{p}^{-1}, \quad \Omega=\Sigma^{-1} \\
& {\left[\boldsymbol{\sigma}^{2} \mid \cdot\right] \propto \prod_{i=1}^{I} \frac{1}{\left(\zeta^{2}+\sigma_{i}^{2}\right) \sigma_{i}^{-1 / 2}}\left\{g\left(\sigma_{i}^{2} \mid \frac{I J}{2}, \frac{\sum_{t=1}^{T} \sum_{j=1}^{J_{i}}\left[\eta_{i t j}-\left(\alpha_{i}+\beta_{i} s_{t}+u_{i j}\right)\right]^{2}}{2}\right)\right\}} \\
& \times \quad\left[\boldsymbol{u} \mid \boldsymbol{\omega}, \boldsymbol{\rho}, \boldsymbol{\gamma}, \boldsymbol{\sigma}^{2}\right],
\end{aligned}
$$

where $g(x \mid a, r)$ is the density of an inverse-gamma distribution with shape $a$ and rate (inverse-scale) $r$;

$$
[\gamma \mid \cdot] \propto\left[\boldsymbol{u} \mid \boldsymbol{\omega}, \boldsymbol{\rho}, \boldsymbol{\gamma}, \boldsymbol{\sigma}^{2}\right]
$$




$$
[\boldsymbol{\omega}, \boldsymbol{\rho} \mid \cdot] \propto \prod_{i=1}^{I} \frac{a}{\left(a+\omega_{i}\right)^{2}}\left[\boldsymbol{u} \mid \boldsymbol{\omega}, \boldsymbol{\rho}, \boldsymbol{\gamma}, \boldsymbol{\sigma}^{2}\right]
$$

Note that the above full conditionals for $\boldsymbol{\sigma}^{2}, \boldsymbol{\gamma}, \boldsymbol{\omega}, \boldsymbol{\rho}$ all depend on the full covariance matrix from the prior distribution of $\boldsymbol{u}$, which involves a matrix determinant and quadratic product. So it is difficult to simplify.

\section{B.2 Full conditional distributions in Chapter 3}

For convenience, we define $\boldsymbol{r}=\left(\psi^{\prime}, \boldsymbol{u}^{\prime}\right)^{\prime}$ and $\boldsymbol{X}=\left(\mathbf{1}_{I J}, \boldsymbol{X}_{\alpha}, \boldsymbol{X}_{\beta}, \boldsymbol{X}_{c}, \boldsymbol{X}_{u}\right)$. Let $q=$ $q_{1}+I$ be the dimension of $\boldsymbol{r}$ where $q_{1}$ is the dimension of $\boldsymbol{\psi}$ and $I$ is the dimension of $\boldsymbol{u}$. Then $\boldsymbol{X}$ is of dimension $D \times q$ where $D=I J$. Based on (3.11), (3.12), (3.13), (3.14), (3.16), (3.18), (3.19) and (3.20), the joint posterior density of $\left(\boldsymbol{v}, \boldsymbol{\psi}, \boldsymbol{\phi}, \boldsymbol{u}, \delta_{0}, \rho, \eta\right)$ given $(\boldsymbol{y}, \boldsymbol{n})$ is

$$
\left[\boldsymbol{v}, \boldsymbol{r}, \delta_{0}, \rho, \eta \mid \boldsymbol{y}, \boldsymbol{n}\right] \propto[\boldsymbol{y} \mid \boldsymbol{n}, \boldsymbol{v}]\left[\boldsymbol{v} \mid \boldsymbol{r}, \delta_{0}\right]\left[\boldsymbol{r} \mid \gamma, \delta_{0}, \rho, \eta\right]\left[\delta_{0}\right][\rho][\eta]
$$

where

$$
\begin{gathered}
{[\boldsymbol{y} \mid \boldsymbol{n}, \boldsymbol{v}] \propto \prod_{d=1}^{D}\left(\begin{array}{c}
n_{d} \\
y_{d}
\end{array}\right) \frac{e^{v_{d} y_{d}}}{\left(1+e^{v_{d}}\right)^{n_{d}}},} \\
{\left[\boldsymbol{v} \mid \boldsymbol{r}, \delta_{0}\right] \propto-\delta_{0}^{-D / 2} \exp \left(-\frac{1}{2 \delta_{0}}(\boldsymbol{v}-\boldsymbol{X} \boldsymbol{r})^{\prime}(\boldsymbol{v}-\boldsymbol{X} \boldsymbol{r})\right)} \\
{\left[\boldsymbol{r} \mid \gamma, \delta_{0}, \rho, \eta\right] \propto-\left|\boldsymbol{\Sigma}_{\boldsymbol{r}}\right|^{-1 / 2} \exp \left(-\frac{1}{2} \boldsymbol{r}^{\prime} \boldsymbol{\Sigma}_{\boldsymbol{r}}^{-1} \boldsymbol{r}\right)} \\
\boldsymbol{\Sigma}_{\boldsymbol{r}}=\left(\begin{array}{cc}
\gamma \boldsymbol{I}_{q_{1}} & 0 \\
0 & \delta(\boldsymbol{I}-\rho \boldsymbol{C})^{-1}
\end{array}\right)_{q \times q}
\end{gathered}
$$


and $\left[\delta_{0}\right],[\rho],[\eta]$ are the same as in (3.18), (3.19) and (3.20). The full conditional distributions used in Gibbs sampling are listed below.

- Let $v_{d}$ be the $d$ th element in vector $\boldsymbol{v}$, then the full conditional distribution of $v_{d}$ given all the other parameters is

$$
\left[v_{d} \mid \cdot\right] \propto \frac{e^{v_{d} y_{d}}}{\left(1+e^{v_{d}}\right)^{n_{d}}} \exp \left(-\frac{\left(v_{d}-\boldsymbol{x}_{d}^{\prime} \boldsymbol{r}\right)^{2}}{2 \delta_{0}}\right)
$$

where $\boldsymbol{x}_{d}^{\prime}$ is the $d$ th row in matrix $\boldsymbol{X}$ and $\cdot$ denotes all the remaining parameters.

- The full conditional distribution of $\boldsymbol{r}$ given all the other parameters is a multivariate normal distribution with mean $\boldsymbol{\Sigma}^{\star} \boldsymbol{X}_{1} \boldsymbol{v}$ and covariance matrix $\delta_{0} \boldsymbol{\Sigma}^{\star}$, where

$$
\boldsymbol{\Sigma}^{\star}=\left(\delta_{0} \boldsymbol{\Omega}_{\boldsymbol{r}}+\boldsymbol{X}_{1}^{\prime} \boldsymbol{X}\right)^{-1} \quad \text { and } \quad \boldsymbol{\Omega}_{\boldsymbol{r}}=\left(\begin{array}{cc}
\gamma^{-1} \boldsymbol{I}_{q_{1}} & 0 \\
0 & \delta^{-1}(\boldsymbol{I}-\rho \boldsymbol{C})
\end{array}\right)_{q \times q}
$$

- The full conditional distribution of $\delta_{0}$ given all the other parameters is InverseGamma distribution with shape parameter equals to

$$
a_{0}+\frac{D+I}{2}
$$

and rate parameter equals to

$$
b_{0}+\frac{1}{2}\left[(\boldsymbol{v}-\boldsymbol{X} \boldsymbol{r})^{\prime}(\boldsymbol{v}-\boldsymbol{X} \boldsymbol{r})+\eta \boldsymbol{u}^{\prime}(\boldsymbol{I}-\rho \boldsymbol{C}) \boldsymbol{u}\right]
$$

Recall that $D$ is the dimension of $\boldsymbol{v}$ and $I$ is the dimension of $\boldsymbol{u}$. 
- The full conditional distribution of $\rho$ given all the other parameters is

$$
\left[\rho \mid \boldsymbol{u}, \eta, \delta_{0}\right] \propto \prod_{i=1}^{I}\left(1-\rho \nu_{i}\right)^{1 / 2} \exp \left(\frac{\rho \eta}{2 \delta_{0}} \boldsymbol{u}^{\prime} \boldsymbol{C u}\right)
$$

where the $\nu_{i}$ s are eigenvalues of $\boldsymbol{C}$.

- The full conditional distribution of $\eta$ given all the other parameters is

$$
\left[\eta \mid \boldsymbol{u}, \delta_{0}, \rho\right] \propto \frac{\eta^{I / 2}}{(\eta+1)^{2}} \exp \left(-\frac{\eta}{2 \delta_{0}} \boldsymbol{u}^{\prime}(\boldsymbol{I}-\rho \boldsymbol{C}) \boldsymbol{u}\right) .
$$

\section{B.3 Full conditional distributions in Chapter 4}

The full conditional distributions of the model parameters are needed to implement a Gibbs sampler.

1. Given $\left(\alpha, \boldsymbol{\psi}, \delta_{0} ; \boldsymbol{y}, \boldsymbol{\Delta}\right)$, the conditional posterior of $v_{i}$ s are independent with densities,

$$
\left[v_{i} \mid \alpha, \boldsymbol{\psi}, \delta_{0} ; \boldsymbol{y}, \boldsymbol{\Delta}\right] \propto \exp \left\{\Delta_{i} y_{i}-e^{v_{i}} y_{i}^{\alpha}-\frac{1}{2 \delta_{0}}\left(v_{i}-\boldsymbol{x}_{i}^{\prime} \boldsymbol{\psi}\right)\right\}, \quad i=1, \ldots, N,
$$

where $\boldsymbol{x}_{i}^{\prime}$ is the $i^{\text {th }}$ row of the covariate matrix $\boldsymbol{X}$.

2. Given $\left(\boldsymbol{v}, \delta_{0}, \eta, \rho ; \boldsymbol{y}, \boldsymbol{\Delta}\right)$ the conditional posterior density of $\boldsymbol{\psi}=\left(\beta_{0}, \boldsymbol{\beta}^{\prime}, \boldsymbol{\theta}^{\prime}, \boldsymbol{w}\right)^{\prime}$ in Model 3 is multivariate normal with mean vector $\boldsymbol{\mu}^{*}$ and covariance matrix $\delta_{0} \Sigma^{*}$, such that

$$
\boldsymbol{\Sigma}^{*}=\left(\delta_{0} \boldsymbol{\Omega}+\boldsymbol{X}_{\alpha}^{\prime} \boldsymbol{X}\right)^{-1}
$$




$$
\boldsymbol{\mu}^{*}=\Sigma^{*} \boldsymbol{X}_{\alpha}^{\prime} \boldsymbol{v}
$$

where

$$
\boldsymbol{\Omega}=\left(\begin{array}{ccc}
\tau^{-1} \boldsymbol{I}_{p+1} & 0 & 0 \\
0 & \tau_{1}^{-1} \boldsymbol{A}_{2} & 0 \\
0 & 0 & \delta^{-1}(\boldsymbol{I}-\rho \boldsymbol{C})
\end{array}\right)
$$

is the size $1+p+T+K$ block-diagonal . For other models, relevant adjustments are needed for the structure of $\boldsymbol{\psi}, \boldsymbol{X}$ and $\boldsymbol{\Sigma}$.

3. Given $\left(\boldsymbol{w}, \eta, \delta_{0}\right)$ the conditional posterior density of $\rho$ is

$$
\left[\rho \mid \boldsymbol{w}, \eta, \delta_{0}\right] \propto \prod_{i=1}^{K}\left(1-\rho \nu_{i}\right)^{1 / 2} \exp \left(\frac{\rho \eta}{2 \delta_{0}} \boldsymbol{w}^{\prime} \boldsymbol{C} \boldsymbol{w}\right)
$$

where the $\nu_{i}$ s are eigenvalues of $\boldsymbol{C}$.

4. Given $(\boldsymbol{v}, \boldsymbol{\psi}, \eta, \rho)$ the conditional posterior density of $\delta_{0}$ is an Inverse Gamma distribution with shape $\tilde{a}_{0}$ and rate $\tilde{b}_{0}$, where

$$
\tilde{a}_{0}=a_{0}+\frac{1}{2}(N+K)
$$

and

$$
\tilde{b}_{0}=b_{0}+\frac{1}{2}\left\{\left(\boldsymbol{v}-\boldsymbol{X}^{\prime} \boldsymbol{\psi}\right)^{\prime}\left(\boldsymbol{v}-\boldsymbol{X}^{\prime} \boldsymbol{\psi}\right)+\eta\left(\boldsymbol{w}^{\prime} \boldsymbol{w}-\rho \boldsymbol{w}^{\prime} \boldsymbol{C} \boldsymbol{w}\right)\right\}
$$

5. Give $\theta$, the conditional posterior distribution for $\tau_{1}$ is an $\operatorname{Inverse-Gamma}\left(\tilde{a}_{1}, \tilde{b}_{1}\right)$, where

$$
\tilde{a}_{1}=a_{1}+\frac{1}{2}(T-2) \quad \text { and } \quad \tilde{b}_{1}=b_{1}+\frac{1}{2} \boldsymbol{\theta}^{\prime} \boldsymbol{A}_{2} \boldsymbol{\theta} .
$$


6. Given $\left(\boldsymbol{w}, \delta_{0}, \rho\right)$ the conditional posterior density of $\eta$ is

$$
\left[\eta \mid \boldsymbol{w}, \delta_{0}, \rho\right] \propto \frac{\eta^{K / 2}}{(\eta+1)^{2}} \exp \left(-\frac{\eta}{2 \delta_{0}} \boldsymbol{w}^{\prime}(\boldsymbol{I}-\rho \boldsymbol{C}) \boldsymbol{w}\right) .
$$

7. Given $(\boldsymbol{v} ; \boldsymbol{y}, \boldsymbol{\Delta})$ the conditional posterior density of $\alpha$ is

$$
[\alpha \mid \boldsymbol{v} ; \boldsymbol{y}, \boldsymbol{\Delta}] \propto \alpha^{\tilde{a}_{\alpha}} \exp \left(-\tilde{b}_{\alpha} \alpha-\sum_{i=1}^{N} y_{i}^{\alpha} e^{v_{i}}\right)
$$

where

$$
\begin{gathered}
\tilde{a}_{\alpha}=a_{\alpha}+\sum_{i=1}^{N} \Delta_{i}-1, \\
\tilde{b}_{\alpha}=b_{\alpha}-\sum_{i=1}^{N} \Delta_{i} \log \left(y_{i}\right) .
\end{gathered}
$$

In Model 5 where we have four different $\alpha$ s for different stages, the density can be easily modified by considering the observations in different stages separately, so that we can sample the four $\alpha$ s separately using similar density functions. 


\section{Bibliography}

American Cancer Society (2014). Colorectal Cancer Facts \& Figures 2014-2016.

Atlanta: American Cancer Society, Inc. 2015 (2014). Breast Cancer Facts \& Figures 2015-2016.

Banerjee, S., Carlin, B. P., and Gelfand, A. E. (2014). Hierarchical modeling and analysis for spatial data. CRC Press.

Bernardinelli, L., Clayton, D., and Montomoli, C. (1995). Bayesian estimates of disease maps: how important are priors? Statistics in Medicine, 14(21-22):24112431.

Besag, J. (1974). Spatial interaction and the statistical analysis of lattice systems. Journal of the Royal Statistical Society. Series B (Methodological), 36(2):192-236.

Besag, J., York, J., and Mollié, A. (1991). Bayesian image restoration, with two applications in spatial statistics. Annals of the institute of statistical mathematics, 43(1):1-20.

Bleicher, R. J., Ruth, K., Sigurdson, E. R., Beck, J. R., Ross, E., Wong, Y.-N., Patel, 
S. A., Boraas, M., Chang, E. I., Topham, N. S., et al. (2016). Time to surgery and breast cancer survival in the united states. JAMA oncology, 2(3):330-339.

Botella-Rocamora, P., Martinez-Beneito, M. A., and Banerjee, S. (2015). A unifying modeling framework for highly multivariate disease mapping. Statistics in medicine, 34(9):1548-1559.

Box, G. E. (1980). Sampling and bayes' inference in scientific modelling and robustness. Journal of the Royal Statistical Society. Series A (General), pages 383-430.

Cadwell, B. L., Thompson, T. J., Boyle, J. P., and Barker, L. E. (2010). Bayesian small area estimates of diabetes prevalence by us county, 2005. J Data Sci, 8(1):173188.

Carlin, B. P., Banerjee, S., et al. (2003). Hierarchical multivariate car models for spatio-temporally correlated survival data. Bayesian statistics, 7:45-63.

Chen, C., Wakefield, J., and Lumely, T. (2014). The use of sampling weights in bayesian hierarchical models for small area estimation. Spatial and spatio-temporal epidemiology, 11:33-43.

Chen, M.-H., Shao, Q.-M., and Ibrahim, J. G. (2012). Monte Carlo methods in Bayesian computation. Springer Science \& Business Media.

Cheng, C.-I. and Speckman, P. L. (2012). Bayesian smoothing spline analysis of variance. Computational Statistics \& Data Analysis, 56(12):3945-3958.

Dai, L., He, Z., Sun, D., and Schootman, M. (2007). Hierarchical models for detecting geographical effects in cancer incidence and survival. Far East Journal of Applied Mathematics, 29(1):1-18. 
Dean, C., Ugarte, M., and Militino, A. (2001). Detecting interaction between random region and fixed age effects in disease mapping. Biometrics, 57(1):197-202.

Ferkingstad, E., Rue, H., et al. (2015). Improving the inla approach for approximate bayesian inference for latent gaussian models. Electronic Journal of Statistics, $9(2): 2706-2731$.

Gelfand, A. E., Dey, D. K., and Chang, H. (1992). Model determination using predictive distributions with implementation via sampling-based methods. Technical report, STANFORD UNIV CA DEPT OF STATISTICS.

Gelfand, A. E. and Vounatsou, P. (2003). Proper multivariate conditional autoregressive models for spatial data analysis. Biostatistics, 4(1):11-15.

Gelman, A. (2007). Struggles with survey weighting and regression modeling. Statistical Science, pages 153-164.

Gelman, A. et al. (2006). Prior distributions for variance parameters in hierarchical models (comment on article by browne and draper). Bayesian analysis, 1(3):515534.

Gelman, A. and Rubin, D. B. (1992). Inference from iterative simulation using multiple sequences. Statistical science, pages 457-472.

Gilks, W. R., Best, N., and Tan, K. (1995). Adaptive rejection metropolis sampling within gibbs sampling. Applied Statistics, pages 455-472.

Goicoa, T., Adin, A., Ugarte, M., and Hodges, J. (2017). In spatio-temporal disease mapping models, identifiability constraints affect pql and inla results. Stochastic Environmental Research and Risk Assessment, pages 1-22. 
Gotway, C. A. and Young, L. J. (2002). Combining incompatible spatial data. Journal of the American Statistical Association, 97(458):632-648.

Greco, F. P. and Trivisano, C. (2009). A multivariate car model for improving the estimation of relative risks. Statistics in medicine, 28(12):1707-1724.

Horvitz, D. G. and Thompson, D. J. (1952). A generalization of sampling without replacement from a finite universe. Journal of the American statistical Association, 47(260):663-685.

Howlader, N., Noone, A., Krapcho, M., Miller, D., Bishop, K., Kosary, C., Yu, M., Ruhl, J., Tatalovich, Z., Mariotto, A., Lewis, D., Chen, H., Feuer, E., and Cronin, K. e. (2017). SEER Cancer Statistics Review, 1975-2014, National Cancer Institute. Bethesda, MD.

Jemal, A., Ward, E. M., Johnson, C. J., Cronin, K. A., Ma, J., Ryerson, A. B., Mariotto, A., Lake, A. J., Wilson, R., Sherman, R. L., Anderson, R. N., Henley, S. J., Kohler, B. A., Penberthy, L., Feuer, E. J., and Weir, H. K. (2017). Annual Report to the Nation on the Status of Cancer, 1975-2014, Featuring Survival. JNCI: Journal of the National Cancer Institute, 109(9).

Jin, X., Banerjee, S., and Carlin, B. P. (2007). Order-free co-regionalized areal data models with application to multiple-disease mapping. Journal of the Royal Statistical Society: Series B (Statistical Methodology), 69(5):817-838.

Jin, X., Carlin, B. P., and Banerjee, S. (2005). Generalized hierarchical multivariate car models for areal data. Biometrics, 61(4):950-961. 
Kimeldorf, G. S. and Wahba, G. (1970). A correspondence between bayesian estimation on stochastic processes and smoothing by splines. The Annals of Mathematical Statistics, 41(2):495-502.

Knorr-Held, L. (2000). Bayesian modelling of inseparable space-time variation in disease risk. Statistics in medicine, 19(17-18):2555-2567.

Lauritzen, S. L. (1996). Graphical models, volume 17. Clarendon Press.

Leroux, B. G., Lei, X., and Breslow, N. (2000). Estimation of disease rates in small areas: A new mixed model for spatial dependence. In Statistical Models in Epidemiology, the Environment, and Clinical Trials, pages 179-191. Springer.

Lumley, T. (2011). Complex surveys: a guide to analysis using R, volume 565. John Wiley \& Sons.

Martinez-Beneito, M. A. (2013). A general modelling framework for multivariate disease mapping. Biometrika, 100(3):539-553.

Martino, S. and Rue, H. (2009). Implementing approximate bayesian inference using integrated nested laplace approximation: A manual for the inla program. Department of Mathematical Sciences, NTNU, Norway.

McGee, S. A., Durham, D. D., Tse, C.-K., and Millikan, R. C. (2013). Determinants of breast cancer treatment delay differ for african american and white women. Cancer Epidemiology and Prevention Biomarkers, 22(7):1227-1238.

Mercer, L., Wakefield, J., Chen, C., and Lumley, T. (2014). A comparison of spatial smoothing methods for small area estimation with sampling weights. Spatial statistics, 8:69-85. 
Pfeffermann, D. et al. (2013). New important developments in small area estimation. Statistical Science, 28(1):40-68.

Raghunathan, T. E., Xie, D., Schenker, N., Parsons, V. L., Davis, W. W., Dodd, K. W., and Feuer, E. J. (2007). Combining information from two surveys to estimate county-level prevalence rates of cancer risk factors and screening. Journal of the American Statistical Association, 102(478):474-486.

Rao, J. N. (2015). Small-Area Estimation. Wiley Online Library.

Riebler, A., Sørbye, S. H., Simpson, D., and Rue, H. (2016). An intuitive bayesian spatial model for disease mapping that accounts for scaling. Statistical methods in medical research, 25(4):1145-1165.

Rosenberg, J., Chia, Y. L., and Plevritis, S. (2005). The effect of age, race, tumor size, tumor grade, and disease stage on invasive ductal breast cancer survival in the u.s. seer database. Breast Cancer Research and Treatment, 89(1):47-54.

Rue, H. and Held, L. (2005). Gaussian Markov random fields: theory and applications. CRC press.

Rue, H., Martino, S., and Chopin, N. (2009). Approximate bayesian inference for latent gaussian models by using integrated nested laplace approximations. Journal of the royal statistical society: Series b (statistical methodology), 71(2):319-392.

Schmaltz, C. and Sun, D. (2010). Marginally conditional autoregressive distributions for misaligned regions. In JSM Proceedings, Section on Bayesian Statistical Science, pages $4984-4998$. 
Schmaltz, C. L. (2012). Marginally modeling misaligned regions and handling masked failure causes with imprecision. PhD thesis, University of Missouri-Columbia.

Si, Y., Pillai, N. S., Gelman, A., et al. (2015). Bayesian nonparametric weighted sampling inference. Bayesian Analysis, 10(3):605-625.

Siegel, R. L., Miller, K. D., and Jemal, A. (2017). Cancer statistics, 2017. CA: A Cancer Journal for Clinicians, 67(1):7-30.

Simpson, D., Rue, H., Riebler, A., Martins, T. G., Sørbye, S. H., et al. (2017). Penalising model component complexity: A principled, practical approach to constructing priors. Statistical Science, 32(1):1-28.

Smith, E. C., Ziogas, A., and Anton-Culver, H. (2013). Delay in surgical treatment and survival after breast cancer diagnosis in young women by race/ethnicity. JAMA surgery, 148(6):516-523.

Smith, R. L. and Naylor, J. (1987). A comparison of maximum likelihood and bayesian estimators for the three-parameter weibull distribution. Applied Statistics, pages 358-369.

Speckman, P. L. and Sun, D. (2003). Fully bayesian spline smoothing and intrinsic autoregressive priors. Biometrika, 90(2):289-302.

Spiegelhalter, D. J., Best, N. G., Carlin, B. P., and Van Der Linde, A. (2002). Bayesian measures of model complexity and fit. Journal of the Royal Statistical Society: Series B (Statistical Methodology), 64(4):583-639.

Sun, D. and Speckman, P. L. (2008). Bayesian hierarchical linear mixed models 
for additive smoothing splines. Annals of the Institute of Statistical Mathematics, 60(3):499-517.

Sun, D., Tsutakawa, R. K., Kim, H., He, Z., et al. (2000). Spatio-temporal interaction with disease mapping. Statistics in Medicine, 19(15):2015-2035.

Sun, D., Tsutakawa, R. K., and Speckman, P. L. (1999). Posterior distribution of hierarchical models using car (1) distributions. Biometrika, 86(2):341-350.

Team, R. C. et al. (2013). R: A language and environment for statistical computing.

Ugarte, M. D., Adin, A., Goicoa, T., and Militino, A. F. (2014). On fitting spatiotemporal disease mapping models using approximate bayesian inference. Statistical methods in medical research, 23(6):507-530.

Vandendijck, Y., Faes, C., Kirby, R. S., Lawson, A., and Hens, N. (2016). Modelbased inference for small area estimation with sampling weights. Spatial Statistics, 18:455-473.

Wingo, P. A., Ries, L. A., Parker, S. L., and Heath, C. W. (1998). Long-term cancer patient survival in the united states. Cancer Epidemiology and Prevention Biomarkers, 7(4):271-282.

Yancik, R., Wesley, M. N., Ries, L. A., Havlik, R. J., Edwards, B. K., and Yates, J. W. (2001). Effect of age and comorbidity in postmenopausal breast cancer patients aged 55 years and older. Jama, 285(7):885-892.

Zhang, S., Sun, D., He, C. Z., and Schootman, M. (2006). A bayesian semi-parametric model for colorectal cancer incidences. Statistics in medicine, 25(2):285-309. 


\section{VITA}

Jiang Du was born on November 23, 1989 in Yan'an, Shaanxi Province in China. He graduated with a B.S. in Information \& Computation Science from the Department of Mathematics in Fudan University, Shanghai, China in 2012. Then he continued his graduate study in the Department of Statistics at the University of MissouriColumbia. He received Master of Arts in Statistics in May, 2014 and PhD in Statistics in May, 2018. 